\title{
Shared mental models and shared temporal cognitions
}

Citation for published version (APA):

Marques dos Santos, C. (2016). Shared mental models and shared temporal cognitions: contributions to team processes and team effectiveness. [Doctoral Thesis, Maastricht University, Instituto Universitário de Lisboa - ISCTE-IUL]. Maastricht University. https://doi.org/10.26481/dis.20160628cm

Document status and date:

Published: 01/01/2016

DOI:

$10.26481 /$ dis. $20160628 \mathrm{~cm}$

Document Version:

Publisher's PDF, also known as Version of record

\section{Please check the document version of this publication:}

- A submitted manuscript is the version of the article upon submission and before peer-review. There can be important differences between the submitted version and the official published version of record.

People interested in the research are advised to contact the author for the final version of the publication, or visit the DOI to the publisher's website.

- The final author version and the galley proof are versions of the publication after peer review.

- The final published version features the final layout of the paper including the volume, issue and page numbers.

Link to publication

\footnotetext{
General rights rights.

- You may freely distribute the URL identifying the publication in the public portal. please follow below link for the End User Agreement:

www.umlib.nl/taverne-license

Take down policy

If you believe that this document breaches copyright please contact us at:

repository@maastrichtuniversity.nl

providing details and we will investigate your claim.
}

Copyright and moral rights for the publications made accessible in the public portal are retained by the authors and/or other copyright owners and it is a condition of accessing publications that users recognise and abide by the legal requirements associated with these

- Users may download and print one copy of any publication from the public portal for the purpose of private study or research.

- You may not further distribute the material or use it for any profit-making activity or commercial gain

If the publication is distributed under the terms of Article $25 \mathrm{fa}$ of the Dutch Copyright Act, indicated by the "Taverne" license above, 




\section{SHARED MENTAL MODELS AND SHARED TEMPORAL COGNITIONS}

Contributions to team processes and team effectiveness 
Printed by Real Panóplia - Produções Gráficas e Serviços de Envelopagem, Lda.

(C) Catarina Marques Santos, Maastricht 2016

All rights reserved. No part of this publication may be reproduced, stored in a retrieval system or transmitted in any form or by any means, electronic, mechanical or by photocopying, recording, or otherwise, without the prior written permission of the author. 


\title{
SHARED MENTAL MODELS AND SHARED TEMPORAL COGNITIONS
}

\author{
Contributions to team processes and team effectiveness
}

\section{DISSERTATION}

to obtain the degree of Doctor at the Maastricht University and Instituto Universitário de Lisboa - ISCTE-IUL, on the authority of the Rector Magnifici, Prof. dr. Luc Soete and Prof. dr. Luís Antero Reto, in accordance with the decision of the Board of Deans, to be defended in public on Tuesday 28 June 2016, at 16 hours.

by

Catarina Marques dos Santos 
Promotor:

Prof. dr. F.R.H. Zijlstra

Co-promotors:

Dr. A.M. Passos (Instituto Universitário de Lisboa - ISCTE-IUL)

Dr. S. Uitdewilligen

Assessment Committee:

Prof. dr. R. Ruiter (Chairman)

Prof. dr. A. Caetano (Instituto Universitário de Lisboa - ISCTE-IUL)

Dr. J. Gevers (Technische Universiteit Eindhoven)

Dr. H. Guenter

Prof. dr. G. Kok

The present doctoral thesis leads to the award of a double doctoral degree between Maastricht University, The Netherlands, and Instituto Universitário de Lisboa - ISCTE-IUL, Portugal.

\section{FCT}

The research project reported in this thesis was financially supported by Fundação para a Ciência e a Tecnologia (Portuguese Foundation for Science and Technology) under the doctoral grant SFRH/BD/79292/2011. 
For André and Tomás 



\section{Contents}

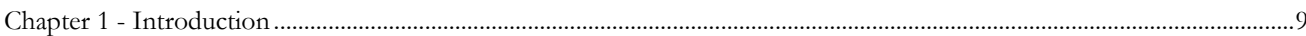

Chapter 2 - Team mental models, relationship conflict and effectiveness over time

Chapter 3 - Why is your team more creative than mine? the influence of shared mental models on intragroup conflict, team creativity and effectiveness.

Chapter 4 - Shared temporal cognitions as substitute for temporal leadership: an analysis of their effects on temporal conflict and team performance

Chapter 5 - When shared cognition leads to closed minds: temporal mental models, team learning, adaptation and performance

Chapter 6 - A temporal common ground for learning: the moderating effect of shared mental models on the relation between team learning behaviors and performance improvement

Chapter 7 - Conclusion

References

Valorization

Appendices

Acknowledgments

Curriculum Vitae 



\section{Chapter 1}

INTRODUCTION 


\section{INTRODUCTION}

Increasingly teams perform intellectual and cognitive tasks that cannot be executed by single individuals (Hinsz, Tindale, Vollrath, 1997; Salas \& Fiore, 2004). Teams perform those complex and demanding tasks in different, and sometimes turbulent, contexts such as organizations, military bases, and hospitals. In order to accomplish the tasks effectively, team members need to use their cognitive resources and cooperate with each other to assess situations, choose the appropriate procedures, solve problems, and make decisions (Cooke, Gorman, Myers, \& Duran, 2012; Salas, Fiore, \& Letsky, 2012). Previous research has shown that it is crucial for effective coordination and team performance that team members have well developed cognitive structures about important aspects of the team's work and that these cognitive structures are aligned among the team members. As team members need to define their strategy and procedures to interact with each other, to work under time pressure to meet tight deadlines, a shared mental model - a common understanding among team members about the task, team and temporal aspects of work (Klimoski \& Mohammed, 1994; Mohammed, Hamilton, Tesler, Mancuso, \& McNeese, 2015) - and a shared temporal cognition - a mental representation of knowledge among team members about time and deadlines (Gevers, Rutte, \& van Eerde, 2006) - are important to foster team effectiveness.

Researchers have shown that shared mental models and shared temporal cognitions play important roles in fostering team effectiveness via functional team processes (i.e., processes that help team members to improve their work and interactions), such as coordination and communication (e.g., Marks, Zaccaro, \& Mathieu, 2000; Mathieu, Heffner, Goodwin, Salas, \& Cannon-Bowers, 2000). Other functional team processes may explain why shared mental models and shared temporal cognitions foster team effectiveness, such as team creativity and team learning. Teams need to be creative in accomplishing their tasks because organizational contexts are increasingly competitive. It can be argued that when teams share a mental model they are more likely to be creative because they coordinate their work, and cooperate with each other; or it can be argued that when teams share a mental model they are less likely to be creative because they do not discuss different points of view. Further, as team members increasingly need to learn from each other in order to adapt quickly to changing environments and maintain high levels of performance, teams may be better able to engage in learning behaviors when their members share a mental model. Thus far, the effect of shared mental models on team creativity and team learning processes is unknown. 
While functional team processes may positively translate the effect of shared mental models and shared temporal cognitions on team effectiveness, dysfunctional team processes, such as intragroup conflict - an incompatible perception among team members regarding interests, ideas or values (DeChurch, Mesmer-Magnus, \& Doty, 2013; Jehn \& Mannix, 2001) may constrain the positive effect of shared mental models and shared temporal cognitions on team effectiveness. As positive interpersonal relations play an important role in linking shared mental models and shared temporal cognitions with team effectiveness (Klimoski \& Mohammed, 1994), team members that share a common understanding about important aspects of work need to experience low levels of conflict in order to achieve high levels of performance.

Teams and team leaders increasingly face a number of temporal challenges in the organizational contexts where they operate. Teams work under time pressure to meet tight deadlines, manage different projects at the same time and coordinate their work with team members that are geographically dispersed (Ancona \& Chong, 1999; O'Leary, Mortenson, \& Woolley, 2011; Waller, Conte, Gibson, \& Carpenter, 2001). Thus, it is important that team members share an understanding about the temporal aspects of work to temporally coordinate their tasks and that team leaders help team members to plan their work and manage their time to accomplish their tasks meeting the deadlines. Otherwise, team members may engage in temporal conflicts - disputes about time, deadlines, and the duration of the tasks (Standifer et al., 2015; Yang, 2009) - and may not achieve their goals. This means that team members need to have share temporal mental models and temporal cognitions, and team leaders need to employ temporal leadership behaviors in order to minimize temporal conflict and promote team performance.

In order to contribute to the understanding that already exist in the team cognition ${ }^{1}$ literature about the effect of shared mental models and shared temporal cognitions on team effectiveness, this thesis focuses on the specific effects of these two cognitive constructs on team processes and team effectiveness. Further, this work focuses on the role of time, both as content and as a dynamic process, to understand the relationship of shared mental models and shared temporal cognitions on team effectiveness over time. In sum, in this thesis I look at important yet unstudied mechanisms (intragroup conflict, team creativity, and team learning) and boundary conditions (temporal leadership) that explain why shared mental models and

\footnotetext{
1 Although we use the term team cognition more often, we use the terms team cognition and shared cognition interchangeably throughout this work.
} 
shared temporal cognitions impact on team effectiveness. Importantly, throughout this thesis team cognition refers to cognitive structures and contents that are shared among team members, and to the cognitive processes in which team members collectively engage over the team lifecycle (i.e., the more stable cognitive structures that people use in their thinking), rather than to the active process of cognition (i.e., what people actually do in terms of thinking and what teams do in terms of discussing and sensemaking) (Monteil \& Huguet, 1999; Uitdewilligen, 2011).

The contributions of this thesis are threefold. First, this work contributes to the shared mental models literature showing that a shared mental model established from the beginning of the team lifecycle helps the team members to interact with each other in an appropriated way without engaging in disruptive relations. Mathieu and Rapp (2009) argue that "many teams jump directly into task performance without taking time to address how they will manage their teamwork and taskwork activities" (p. 90). This thesis shows that when team members take time to develop a solid foundation early in the team lifecycle about the way they should manage their teamwork they are able to minimize intragroup conflict, to be creative, to engage in team learning behaviors, and in turn achieve their goals.

Second, this thesis contributes to both the shared mental models and team processes literature by providing insights into the way a shared understanding about task, team, and temporal aspects of work are related to team processes (intragroup conflict, team creativity, and team learning), and team effectiveness. Wildman and colleagues (2012) noted that "very few studies are examining the affective, behavioral, and cognitive processes affected by team knowledge constructs" (p. 104). In fact, a small number of researchers have analyzed the effect of shared mental models and shared temporal cognitions on team processes, such as coordination, communication, and backup behaviors, and in turn on team effectiveness (Marks et al., 2000; Marks, Sabella, Burke, \& Zaccaro, 2002; Mathieu et al., 2000). It is, as yet, unclear what role other important team processes play in translating the knowledge team members share into team effectiveness. This thesis provides two perspectives about the effect of shared mental models and shared temporal cognitions on these unexplored team process mechanisms. On one hand, this works shows that these cognitive constructs are beneficial for team functioning because team members avoid conflicting situations and have creative ideas, and shared mental models translate team learning behaviors into team performance improvement. On the other hand, this work shows that shared mental models are harmful for team functioning when team members share incorrect knowledge because they do not learn 
from each other and do not discuss the errors they make or the problems that occur over the team lifecycle.

Third, this thesis contributes to team research by integrating time, as content, in team constructs and relating them with shared mental models and shared temporal cognitions. As teams are increasingly pressured by time, it is crucial to understand how teams can successfully perform their tasks while they are able to temporally coordinate and overcome a number of temporal challenges. This work advances knowledge on temporal research by showing that temporal mental models are important for teams to coordinate their activities according to the time schedule: shared mental models and shared temporal cognitions help teams to minimize the level of temporal conflict and to promote team effectiveness; temporal leadership diminish the level of temporal conflict and facilitate team performance; and shared temporal cognitions substitute for temporal leadership in reducing the level of temporal conflict. Further, this thesis contributes to temporal research by analyzing the effect of shared mental models and shared temporal cognitions on team processes, and team effectiveness in different time moments; and by analyzing the joint effect of shared mental models and team learning processes on team performance over time. This thesis shows that, indeed, teams have distinct needs over their lifecycle and that having a shared understanding about important aspects of teamwork has consequences over the team lifecycle.

This work is organized as follows: I begin by defining what I mean with teams and teamwork, and I will present commonly used models that explain the main mechanisms and factors that influence team effectiveness. Then, building on cognitive psychology, I will explain how individuals acquire and use knowledge, how the cognition construct was adopted by social and organizational researchers, and how teams build a shared representation of knowledge. Next, I will focus on shared mental models and shared temporal cognitions conceptualizing these two cognitive constructs, and explaining what it is known and what is not known about the influence of shared mental models and shared temporal cognitions on team effectiveness. I present five empirical studies in which, using four samples and diverse statistical analysis, I investigate the effect of shared mental models and shared temporal cognitions on team processes and effectiveness, as well as a number of boundary conditions and intervening mechanisms. Finally, I close this thesis with a general discussion where I discuss the theoretical and practical implications of this work, limitations and directions for future research. 


\section{TEAMS, TEAMWORK AND TEAM EFFECTIVENESS}

History is full of examples that show failures in teamwork that led to medical errors, military air crashes, and industrial disasters. The USS Vincennes incident in 1988, the Space Shuttle Challenger disaster in 1986, and the failures on organizational answers to Hurricane Katrina in 2005 are some of the examples. In those situations, errors related to communication, coordination, team leadership, decision-making, and incongruence in temporal perceptions led to terrible personal and material losses and to tragic consequences (Kozlowski \& Ilgen, 2006; Moynihan, 2007; Salas, Stagl, Burke, \& Goodwin, 2007). Over the years, researchers have made efforts to understand how teams can effectively work together, manage team interaction, and achieve their goals avoiding such disastrous situations. In this chapter I provide definitions of teams and teamwork, I explain the commonly used team effectiveness models and frameworks, and I identify a number of mechanisms and contextual factors that influence team effectiveness.

\section{Teams and teamwork}

Over the last years, the way work is structured within organizations has changed dramatically. While in the past the job was mainly performed by individuals working in functionalized structures, nowadays tasks are executed by teams that are embedded in complex systems (Devine, Clayton, Phillips, Dunford, \& Melner, 1999; Hackman, 2012; Kozlowski \& Ilgen, 2006; McGrath, Arrow, \& Berdahl, 2000; Salas et al., 2007). As work has become increasingly complex, demanding and challenging, organizations rely on teams to perform the work because teams are able to innovate, adapt their strategies, and improve their performance under time pressure, ensuring that organizations compete with others and survive in challenging environments (Cronin, Weingart, \& Todorova, 2011; Marks, Mathieu, \& Zaccaro, 2001; McGrath et al., 2000; Burke, Stagl, Salas, Pierce, \& Kendal, 2006a; Kozlowski \& Ilgen, 2006; Salas et al., 2007). Thus, teamwork is preferred over individual work when tasks are too complex for the individual's capacity, when errors may cause dangerous consequences, when multiple decisions are needed to make quickly, and when someone's lives depend on the combined knowledge of individual members (Salas, Cooke, \& Rosen, 2008).

Salas, Dickinson, Converse, and Tannenbaum (1992) defined a team as a complex and dynamic system where two or more individuals perform relevant and interdependent 
tasks, share common goal(s), interact socially, and maintain and manage boundaries. In a team, members have different knowledge and skills, and roles are distributed among them. Further, a team has a hierarchical structure, has a limited life span and is an emergent and adaptive entity (Kozlowski \& Ilgen, 2006) "embedded within an organizational/environmental context that influences and is influenced by ongoing processes and performance outcomes" (Salas et al., 2007, p. 189). This means that a team is characterized by multiple, bidirectional, and nonlinear relations where team members know who is on the team, and who is not, and socially interact over time either face-to-face, or through virtual communication methods when team members are geographically dispersed (Hackman, 2012; Kozlowski \& Bell, 2003). Moreover, all team members depend of each other, aim to and have the responsibility to achieve the shared goals by working together, cooperating and interacting with each other to complete the inter-related tasks, at least the necessary time to accomplish the goals (McGrath et al., 2000; Salas et al., 1992). In teams, diversity in expertise and knowledge creates optimal conditions for performing tasks that could not be executed by individuals (Salas, Rosen, Burke, \& Goodwin, 2009). Regarding the hierarchical structure, leadership functions may be employed by a single member, or shared by formally or informally designated multiple members (Carson, Tesluk, \& Marrone, 2007; Pearce \& Sims Jr., 2002; Zaccaro, Rittman, \& Marks, 2001). Finally, a team is linked and embedded in a multilevel system composed of individuals, teams, and organizations that influences the team processes in which a team engages because it drives "the difficulty, complexity, and tempo of the team task" (Kozlowski \& Ilgen, 2006, p. 80).

Teamwork is "the means by which individual task expertise is translated, magnified, and synergistically combined to yield superior performance outcomes” (Salas et al., 2009, p. 42). Teamwork comprises behaviors, cognitions, as well as knowledge, skills, and attitudes (KSAs) that facilitate coordination and the adaptive performance in order to lead the team to achieve its goals and mission (Baker, Day, \& Salas, 2006; Salas et al., 2007). Researchers have identified, for instance, teammate characteristics, role responsibilities, team mission, objectives, and resources as required knowledge competencies (Cannon-Bowers, Tannenbaum, Salas, \& Volpe, 1995); mutual performance monitoring, feedback, coordination, and communication as required skills (Cannon-Bowers et al., 1995; McIntyre \& Salas, 1995); and team orientation, shared vision, collective efficacy, and mutual trust as required attitudinal competencies (Cannon-Bowers et al., 1995; Salas et al., 2007). 
Chapter 1

\section{Team effectiveness}

If you ask team leaders or managers whether their team is effective or ineffective, they are likely to take into account whether the team achieves high levels of performance or not. However, besides performance there are other criteria to classify a team as effective or ineffective (Hackman, 1987). Sometimes, even when a team achieves high levels of performance, team members are not satisfied with the team experience, or do not want to continue to work with the same team in the future. Thus, it is important to understand whether the experience of working with the team over its lifecycle contribute to satisfaction and well-being of team members, as well as for their willingness to continue to work together. Those three criteria to analyze team effectiveness were proposed by Hackman (1987): performance, satisfaction and viability.

Team performance is the objective criterion that indicates team level task accomplishment. In particular, team performance refers to the degree to which the task output fulfills or exceeds the quality and quantity standards of those who receive, review or use the output (Hackman, 1987). Team satisfaction refers to the feeling that the team work experience positively contributes to their overall well-being and growth (Hackman, 1987). Team viability was conceptualized by Hackman (1987) as a willingness to continue working with the same team members. Over the years, based on Hackman' definition of team viability, different conceptualizations of team viability have emerged in literature. A number of researchers have added some components to the construct, such as, satisfaction (Resick, Dickson, Mitchelson, Allison, \& Clark, 2010a; Sundstrom, De Meuse, \& Futrell, 1990), participation (Resick et al., 2010a), and attachment to the team (Balkundi \& Harrison, 2006). All those different conceptualizations of team viability have led to construct confusion, and to the inability to distinguish team viability from other related constructs, such as, team satisfaction and team cohesion (Bell \& Marentette, 2011; Mathieu, DeShon, \& Bergh, 2008a). In this thesis we followed Hackman's definition and conceptualized team viability as a willingness to continue working together over team episodes. This definition ensures that we only measure team viability, instead a mix of team viability and team satisfaction (Sundstrom et al., 1990). 


\section{Team effectiveness models and frameworks}

Team effectiveness is influenced by different individual, team, and organizational factors, as well as, by contextual factors external to teamwork's environment (Marks et al., 2001; Mathieu, Maynard, Rapp, \& Gilson, 2008b; Salas et al., 2007). A number of models and frameworks have been developed over the years by team researchers in order to understand which factors contribute to team effectiveness, and why some teams are more effective than others (e.g., Gladstein, 1984; Hackman, 1987; Ilgen, Hollenbeck, Johnson, \& Jundt, 2005; Marks et al., 2001; Mathieu et al., 2008b; Salas, Sims, \& Burke, 2005; Salas et al., 2007). Importantly, effective teams require both taskwork and teamwork (Baker et al., 2006; Marks et al., 2001). Taskwork refers to the interactions team members establish with task, equipment, and system in order to accomplish the team's goals (Marks et al., 2001; Mathieu et al., 2008b). Teamwork refers to the interactions between team members that allow them to understand how they accomplish the team's task with each other, and how they coordinate their efforts sharing knowledge about the team's resources, goals and the work environment constraints (Marks et al. 2001; Mathieu et al., 2008b). In the next paragraphs we describe the team effectiveness models and framework that influence and are the foundations of this work.

McGrath (1964) was one of the first researchers to propose a framework to study team effectiveness, based on the logic of input-process-output (I-P-O). In this framework, imputs refer to antecedent factors at individual, team, and organizational level that enable and constrain task performance and team members' interaction, such as, team member characteristics, team leadership, material and human resources, and environmental context (Kozlowski \& Ilgen, 2006; Mathieu et al., 2008b). Processes refer to interdependent actions in which team members engage, that allow task accomplishment through the combination of individual resources, knowledge and skills (Kozlowski \& Ilgen, 2006; Mathieu et al., 2008b). According to the I-P-O framework, processes act as mediational factors that explain how team inputs are transformed into team outputs (Mathieu et al., 2000; 2008b). Outputs result from the teamwork and comprise performance, satisfaction, and viability (Kozlowski \& Ilgen, 2006; Mathieu et al., 2008b).

Over the years, researchers pointed out some weaknesses of the I-P-O framework and updated it in order to be appropriated for study teams defined as dynamic, complex, and adaptive entities (e.g., Burke et al., 2006a; Marks et al., 2001; Rosen et al., 2011; Salas et al., 2007). This framework implies a single and linear cycle from inputs to outcomes that does not 
consider the possibility of feedback loops, does not explain the reciprocal influences of inputs, processes and outcomes over time, and does not capture the complex and dynamic nature of teams (Ilgen et al., 2005). Furthermore, the I-P-O model postulates that the mediating factors that translate inputs into outputs are only team processes.

Marks and colleagues (2001) developed a temporally based framework following the assumption that teams work in temporal cycles oriented to tasks goals, which they called episodes - "distinguishable periods of time over which performance accrues and feedback is available" (Marks et al., 2001, p. 359). Episodes may vary in their length, and may be divided in sub-episodes with restricted objectives and time limited that contribute to the larger team goal (Marks et al., 2001). Team performance happens in a sequence of I-P-O episodes that occur simultaneously and sequentially: when an episode ends, another one starts (i.e., outcomes from the previous episode act as an input for the next one). Importantly, Marks and colleagues (2001) distinguished team processes from emergent states. They noted that not all the variables classified as processes in the I-P-O framework by McGrath (1964) were, in fact, processes. Team processes refer to 'members' interdependent acts that convert inputs to outcomes through cognitive, verbal, and behavioral activities directed toward organizing taskwork to achieve collective goals" (Marks et al., 2001, p. 357). Emergent states are dynamic characteristics of a team that vary and evolve over time in reaction to changes to the team context, inputs, processes and outcomes, and that impact team performance (Marks et al., 2001; Mathieu et al., 2008b). Thus, whereas team processes represent team interaction or team actions that lead teams to achieve their outcomes, emergent states result from team experience, become new inputs to succeeding processes and outcomes, and do not represent team interaction or team actions (Marks et al., 2001).

Based on the episodic approach and on the recurring phase model of team processes, the temporally based framework postulates that teams fulfill different processes at different times and use them simultaneously over the performance episodes and, in a cyclical way, depending on the task they are accomplishing, and on the phase they are (Marks et al., 2001; Mathieu et al., 2008b). During the episodes, teams may alternate between transition phases and action phases (Marks et al., 2001). In transition phases teams reflect on past performance, and plan the activities that guide future performance and ensure goal achievement, in action phases teams are focused on activities that directly contribute to goal achievement (Marks et al., 2001). Over these two phases teams may engage in three different types of team processes (Marks et al., 2001): transition, action, and interpersonal processes. Transition processes 
comprise three sub-processes (mission analysis, goal specification, strategy formulation and planning) through which the team analyze past performance, evaluate their activities, and plan the future direction in order to accomplish the team goal (Marks et al., 2001). Action processes comprise four sub-processes (monitoring progress toward goals, systems monitoring, team monitoring and backup, and coordination) through which teams develop activities that directly lead to team goal achievement (Marks et al., 2001). Interpersonal processes comprise three sub-processes (conflict management, motivating and confidence building, and affect management) through which teams manage interpersonal relationships (Marks et al., 2001). The transition and action processes have a "natural temporal rhythm" and are more likely to occur during transition or action phases (Marks et al., 2001, p. 369). Interpersonal processes may occur throughout transition and action phases. In short, this framework postulates that "processes occur over and over again during team episodes, influenced by inputs (including emergent states) and influencing proximal outcomes (also including emergent states) within action and transition phases and across tasks and time as teams move toward goal accomplishment” (Marks et al., 2001, p. 361).

Later, Ilgen and colleagues (2005) developed an input-mediator-output-input (IMOI) model to overcome the limitations of the classic framework of McGrath (1964) that we mentioned above. Researchers replaced processes ("P") by mediators ("M") to reflect the broader range of mediational variables that may transmit the effect of inputs to outputs (i.e., team processes and emergent states). Researchers added an extra input ("I") at the end of the model to reflect the cyclical causal feedback as a final output is a new input and consequently originates a new performance episode. This model also states that the relationship between variables may be nonlinear or conditional (Ilgen et al., 2005).

Related to IMOI model, and based on updated versions of I-P-O framework, Mathieu, and colleagues (2008b) advanced an Input-Mediator-Outcome (IMO) model. This model postulates that inputs comprise organizational context, team context, and members. Between those three components there is a multilevel influence in which the higher level factors (e.g., organizational structure and functioning in organizational context) influence lower level factors (e.g., leadership behaviors, task design, and team members' interaction and communication in team and individual context), and vice-versa (Mathieu et al., 2008b). The mediators include both team processes, and emergent states. Finally, the outcomes include multiple criteria, such as, performance and customer service (Mathieu et al., 2008b). Similar to the IMOI model, the IMO model represents the feedback loops from outcomes to mediators, 
Chapter 1

from outcomes to inputs, and from mediators to inputs. This model combines two distinct approaches: the developmental and the episodic. The developmental approach reflects how teams change and how they are influenced by different factors while they mature over time; and the episodic approach postulates that teams engage in different processes at different moments of the team lifecycle (Mathieu et al., 2008b).

This thesis is mainly based on the temporally based framework by Marks and colleagues (2001), as teams work in a sequential of I-P-O episodes. Although the criticism researchers have made to the I-P-O model, this temporal framework is more than a combination of I-P-O episodes as defined by McGrath (1964). The temporally based framework considers the developmental and the episodic approaches, which consider that teams change over time, are influenced by different factors, and execute different team processes over time and at different times (Marks et al., 2001; Mathieu et al., 2008b). This framework also states that both team processes and emergent states may act as mediating factors that translate the effects of inputs into outcomes. Furthermore, emergent states influence team processes, which in turn are likely to alter subsequent emergent states, and "this cyclical pattern continues until teams reach more distal team outcomes" (Marks et al., 2001, p. 358). Those aspects influence the way this work was designed and conducted.

\section{Team mechanisms and contextual factors that influence team effectiveness}

Team effectiveness is influenced by a number of team mechanisms. In particular, team effectiveness is influenced by (a) action and behavioral processes and emergent states, (b) interpersonal, motivational and affective processes and emergent states, and (c) cognitive processes and emergent states (Kozlowski \& Ilgen, 2006). Action and behavioral processes and emergent states refer to acts or team properties that contribute to task and goal accomplishment, such as coordination, cooperation, communication (Kozlowski \& Ilgen, 2006; Marks et al., 2001), and adaptation (Maynard, Kennedy, \& Sommer, 2015). Interpersonal, motivational and affective

processes and emergent states "capture motivational tendencies, relations among team members, and affective reactions" (Kozlowski \& Ilgen, 2006, p. 87) and include emergent states, such as team cohesion (Kozlowski \& Chao, 2012a), group potency (Shea \& Guzzo, 1987), team work engagement (Costa, Passos, \& Bakker, 2014), and team processes such as intragroup conflict (Jehn, 1995; Marks et al., 2001). Cognitive processes and emergent states refer to the shared cognitive structure and organization of knowledge and to the acquisition of 
knowledge that guide interactions among team members that are relevant for the task (Cannon-Bowers \& Salas, 2001; Kozlowski \& Ilgen, 2006). Shared mental models (Marks et al., 2001; Mathieu et al., 2000), transactive memory system (DeChurch \& Mesmer-Magnus, 2010; Lewis, 2003; Marques-Quinteiro, Curral, Passos, \& Lewis, 2013), and shared temporal cognitions (Gevers et al., 2006) refer to cognitive emergent states (Kozlowski \& Ilgen, 2006), while knowledge acquisition refers to a cognitive team process (Kozlowski \& Chao, 2012b).

This work focuses on the cognitive emergent states that contribute to team effectiveness, in particular on shared mental models (i.e., a shared understanding among team members regarding the relevant aspects of the teamwork; Klimoski \& Mohammed, 1994) and on shared temporal cognitions (i.e., a shared understanding about the temporal aspects of the teamwork; Gevers et al., 2006). Throughout this work these cognitive aspects are related with a number of interpersonal and behavioral team processes (intragroup conflict, creativity, and team learning), and outcomes (team performance, satisfaction, and viability). Further, by focusing on the temporal aspects of team functioning and by integrating temporality in team constructs, the interaction role of temporal leadership and shared temporal cognitions on performance via temporal conflict is analyzed.

Both shared mental models and shared temporal cognitions refer to two aspects of team cognition that are bottom-up emergent states (DeChurch \& Mesmer-Magnus, 2010a; Kozlowski \& Klein, 2000). They are bottom-up constructs because they are originating at the individual level (e.g., mental model) and emerge to a higher level to shape a collective phenomenon (e.g., shared mental model) (Costa et al., 2013; Kozlowski \& Klein, 2000). They are a composition (vs. a compilation) form of emergence as the lower level characteristics are similar and emerge to a higher level property (team cognition) that is the same as its individual components (individual cognition) (Kozlowski \& Chao, 2012b). Thus, the knowledge is shared among team members. Shared mental models and shared temporal cognition are emergent states because they take time to develop, may change over time in reaction to changes in the team context, input processes, and outcomes, and depend on how team members collectively interact (Cronin et al., 2011; Marks et al., 2001).

Intragroup conflict, team creativity, and team learning refer to team processes - team actions and interactions that lead the team to achieve their outcomes (Marks et al., 2001). Intragroup conflict occurs when a number of persons within a team perceive differences about interests, values or resources, or perceive incompatible wishes or interests (DeChurch et al., 2013; Jehn \& Mannix, 2001). Three different types of intragroup conflict have been 
identified and analyzed in the literature: task, relationship, and process conflict Jehn, 1995; 1997). Task conflict refers to disagreements among team members related to the content of the tasks, such as differences regarding ideas or opinions (Jehn, 1995). Relationship conflict refers to disagreements based on personal and social issues that are not directly related to the task and that involve negative emotions and tension (De Dreu \& Van Vianen, 2001; Jehn, 1995). Process conflict concerns to disagreements related to each other responsibilities, task delegation, and how the task should be accomplished (Jehn, 1997). Recently, scholars added a fourth type of conflict - temporal conflict - which refers to intragroup disputes about time, the duration of a task, and the length of time the team should spend on a specific task or goal (Mohammed \& Nadkarni, 2011; Standifer et al., 2015). In order to ensure that teams perform well, constructive conflict (i.e., task conflict) need to be maximized, and disruptive conflicts (i.e., relationship, process, and temporal conflict) need to be minimized (DeDreu \& Van Vianen, 2001; de Wit, Greer, \& Jehn, 2012; Jehn, 1995, 1997; Standifer et al., 2015).

Creativity refers to the process of "coming up with fresh ideas for changing products, services, and processes so as to better achieve the organization's goals" (Amabile, Barsade, Mueller, \& Staw, 2005, p. 367). Team creativity is the basis of innovation, and an important booster to team effectiveness (Gilson et al., 2005; Mathieu et al., 2008b). Particularly, team creativity fosters team and organizational innovation and effectiveness (Amabile, 1988; DiLiello, Houghton \& Dawley, 2011) when team members "are open to new ideas, constructively challenge one another, effectively communicate and provide feedback, successfully manage conflict, trust and help each other, and share a commitment to their work" (DiLiello et al., 2011, p. 155).

Team learning process involves a number of behaviors in which teams engage, such as ask questions, discuss and reflect errors or unexpected outcomes, and experiment new working methods (e.g., Edmondson, 1999; Savelsbergh, van der Heijden, \& Poel, 2009). Team learning refers to a bottom-up emergent phenomenon that originates at the individual level and emerges through team member interactions, as a team-level construct (Costa et al., 2013; Kozlowski \& Chao, 2012b; Kozlowski \& Klein, 2000). This means that the individual knowledge that team members acquire is transmitted and propagated across team members through the processes of communication, exchange, observation, and collaboration (Kozlowski \& Bell, 2008; Kozlowski \& Chao, 2012b). Then, the outcomes of these team learning processes emerge as a pool of knowledge as well as explicit and implicit routines shared among the members of the team (Kozlowski \& Bell, 2008). Team learning is a crucial 
team process for organizational teams because team members need to learn from each other, to improve the way they work in order to continuously adapt to the changing environments and maintain high levels of performance (Edmondson, Dillon, \& Roloff, 2007; Rosen et al., 2011; Savelsbergh, Gevers, van der Heijden, \& Poell, 2012).

Although in this thesis the effect of shared mental models or shared temporal cognitions on team adaptation is not analyzed, the effect of team learning on team adaptation is. Team adaptation may be conceptualized as a performance construct, an individual difference construct, a change in performance, and as a process (Baard, Rench, \& Kozlowski, 2014), throughout this work we refer and analyze team adaptation as a process that is linked to team performance (Burke et al., 2006a; Rosen et al., 2011). Accordingly, team adaptation, occurs when an individual or team recognize that a change happens in the team environment, understand that some response or adjustment to team processes need to be done in order to overcome the obstacles and effectively address the unexpected situation (Baard et al., 2014; Maynard et al., 2015). Team adaptation is increasingly important for teams that perform in complex and demanding contexts as they often need to adapt quickly and appropriately to recurrent changes adjusting their cognitive and behavioral processes, and evaluating and analyzing the situations in short periods of time (Baard et al., 2014; Burke et al., 2006a; Rosen et al., 2011; Uitdewilligen, Waller, \& Pitariu, 2013).

Time plays an important role in teamwork as teams work together, evolve, and face temporal challenges over their lifecycle (Kozlowski \& Bell, 2003). Nevertheless, time has been a neglected issue in team research and organizational research (Kozlowski \& Bell, 2003; Roe, 2009). Teams often need to work under tight deadlines, manage multiple projects at the same time, and coordinate their work with team members that are virtually and geographically dispersed (Ancona \& Chong, 1999; Gevers et al., 2006; Hinds \& Mortenson, 2005; O’Leary et al., 2011; Waller et al., 2001). However, as researchers have not considered time in their studies, they cannot provide teams and team leaders with tools that help them to face these challenges, and to manage the activities they need to perform in the time they have (Roe, 2009). Over the last years, researchers have conducted research that explicitly incorporates temporal elements in terms of content into team (cognition) constructs integrating timerelated aspects (e.g., Mohammed \& Nadkarni, 2011; Mohammed et al., 2015; Standifer et al., 2015). Mohammed and Nadkarni (2011) conceptualized temporal leadership and showed that it improves team performance. Temporal leadership emerged from the need to combine temporal activities and team leadership (Ancona, Goodman, Lawrence, \& Tushman, 2001; 
Chapter 1

Halbesleben, Novicevic, Harvey, \& Buckley, 2003), and refers to the behaviors that team leaders employ to help the team to finish the (sub)tasks on time, and to verify whether the team members are doing what they are supposed to be doing (Mohammed \& Nadkarni, 2011). Standifer and colleagues (2015) demonstrated that shared temporal cognitions improve team satisfaction by reducing temporal conflict. More recently, Mohammed and colleagues (2015) operationalized temporal mental models showing that this mental model dimension predict team performance beyond task and team mental models.

To summarize, teams have been the solution for accomplishing complex and dynamic tasks that individuals alone are not able to accomplish. Throughout the team lifecycle, team members engage in team processes in order to achieve the team goals successfully. Based on the temporal based framework (Marks et al., 2001), in this work is analyzed how cognitive emergent states and temporal leadership contribute to team effectiveness via a number of team processes. Further, the combined effect of different temporal constructs on team processes and performance is analyzed.

\section{FROM INDIVIDUAL COGNITION TO TEAM COGNITION}

At the individual level, a mental model refers to a cognitive mechanism that allows humans to generate descriptions of the system and predictions of future system states (Rouse \& Morris, 1986). Understanding how cognition works at the individual level, how individuals process information, solve problems and make decisions could improve team researchers' insights regarding the way team cognition emerges, develops and updates over time. In fact, researchers have called attention to the need to understand team cognition through cognitive processes, namely attention, detection, reasoning, and decision making, in the same way individual cognition is understood (Wildman et al., 2012). In this chapter I build on the cognitive science perspective to explain how people process information, and to distinguish mental models from schemas. I explain how and why social and organizational psychologists integrate cognitive psychology in their theories and research. Further, I explain how teams are able to integrate the individual mental models in a shared mental model. 


\section{Cognition}

Cognition refers to the acquisition and use of knowledge, and involves a number of mental processes that allow the transformation, reduction, elaboration, storage, recovering, and use of sensory inputs (i.e., information) (Reed, 2000). Cognition is the main subject of cognitive psychology, a branch of psychology that studies these mental operations in order to analyze and understand "the processes by which the individual minds perceive, manipulate and interpret information" (Levine \& Resnick, 1993, p. 586). Cognitive psychologists are interested in understanding, for instance, how people solve problems and make decisions. More recently, cognitive psychology has influenced the study of teams, and a number of cognitive psychology's constructs have been used to understand how teams can improve team performance (Salas et al., 2012). Cognitive psychology theories and research paradigms have had a relevant impact on a wide variety of areas, such as, applied cognitive psychology and social cognition (Monteil \& Huguet, 1999; Reed, 2000). Applied cognitive psychology refers to the use of methods and theories from cognitive psychology to understand how cognitive phenomena occur in human life, and to solve practical problems in different areas, such as, education, business, medicine, and industry (Hoffman \& Deffenbacher, 1992). Social cognition concerns to the social processes of encoding, storage, retrieval and manipulation of information used to generate a group-level product (Fiore \& Salas, 2004; Klimoski \& Mohammed, 1994; Monteil \& Huguet, 1999).

\section{Representation of knowledge in memory: mental models and schemas}

Cognitive science aims to understand the representation of knowledge in memory, as well as the structural and processing aspects of knowledge (Rumelhart \& Ortony, 1977). This knowledge may be used by researchers from different areas, for instance, artificial intelligence, cognitive psychology, and human factors, to improve the way people work (Brewer, 2003; Rumelhart \& Ortony, 1977).

Knowledge can be represented in memory through cognitive frameworks. The most studied ways to represent knowledge in memory refer to mental models and schemas. Mental models are "the mechanisms whereby humans are able to generate descriptions of system purpose and form, explanations of system functioning and observed system states, and predictions of future system states" (Rouse \& Morris, 1986, p. 351). Schemas refer to cognitive 
data structures that represent the attributes of generic concepts stored in memory and the relationships among those attributes (Fiske \& Taylor, 1984; Rumelhart \& Ortony, 1977).

Mental models are widely applied in the area of cognitive science, which results of the combination between cognitive psychology and computer science or artificial intelligence (Rouse \& Morris, 1986). Mental models have their roots in the physical sciences, in particularly on the model-based approach to science (Brewer, 2003), that postulates that people make a mental construction of themselves based on mechanical models. Mental models are built by people based on their life experiences and perceptions, and they have an analogical relation with the events that occur in the world (Brewer, 2003). Through mental models, people are able to make inferences and predictions, understand phenomena, experience events and decide what to do (Johnson-Laird, 1983). Consequently, mental models are the basis for reasoning, decision-making and behavior (Jones, Ross, Lynam, Perez \& Leitch, 2011). Mental models can refer either to long-term knowledge, as they represent the model-based knowledge, or to short-term knowledge, as they represent temporary mental constructions based on the understanding of particular events in the world (Brewer, 2003; Johnson-Laird, 1983). Furthermore, mental models may represent both physical and conceptual entities (Johnson-Laird, 1983; Jones et al., 2011). As mental models are an effective mechanism to explain and understand complex performance, this construct has been used by researchers from different areas, such as psychology and engineering, to understand human cognitive functioning and human-system performance (Cannon-Bowers, Salas, \& Converse, 1993).

The term schema was previously used in philosophy by Immanuel Kant, in developmental psychology by Jean Piaget, and in cognitive science by the psychologist Frederic Bartlett (Brewer, 1999). Schemas play an important role in artificial intelligence because machines need to be provided with large amounts of knowledge in order to be intelligent and much of human intelligent behaviors result from the use of knowledge (Brewer, 1999; Rumelhart \& Ortony, 1977). Schemas are related to information processing and are used to represent the concepts stored in memory that helps to organize and interpret information (Fiske \& Taylor, 1984; Rumelhart \& Ortony, 1977). Those concepts may refer, for instance, to objects, situations, and events. Schemas are important to simplify people's understanding of their social world and to select, interpret, and integrate information of other people or situations based on general cases, because they allow people to take shortcuts in interpreting a large amount of information available in their environment (Cannon-Bowers et al., 1993; Fiske 
\& Taylor, 1984). Through schemas people memorize and represent old information in memory, perceive new information, and make inferences and assimilate the new information with the existing knowledge (Fiske \& Taylor, 1984).

Although both schemas and mental models are cognitive structures that represent knowledge in memory there are differences between these concepts. These differences are based on three criteria: structure, flexibility, and knowledge representation (Jones et al., 2011). First, while schemas reflect a static structure of data in memory, mental models use data in a dynamic way (Jones et al., 2011; Rutherford \& Wilson, 2004). Second, schemas refer to inflexible structures of knowledge stored in long-term memory that offer predictive knowledge for routine situations. In contrast, mental models are flexible knowledge structures that combine multiple structures of knowledge to represent a non-routine or unfamiliar situation (Holland, Holyoak, Nisbett, \& Thagard, 1986; Jones et al., 2011). Thus, schemas are only stored in long-term memory, and mental models may be constructed at the time people store information (Brewer, 2003). Third, schemas refer to generic knowledge structures that reflect old generic information, whereas mental models are specific knowledge structures that represent new situations based on generic knowledge, such as space, time and causality, and that are built at the time of input (Brewer, 1987; Jones et al., 2011). In addition, whereas schemas are rigid knowledge structures, mental model are manipulable because people are able to "convert abstract logical problems into specific models and then manipulate the information in the models to generate conclusions" (Brewer, 2003, p. 2). This allows people to predict states through the mental manipulation of mental parameters, which lead them to create causal links and predict a number of options for a possible decision previous to the decision making. Thus, people are able to anticipate the possible outcomes of their choices (Cannon-Bowers et al., 1993).

\section{The emergence of team cognition}

When cognitive psychology research emerged, psychologists mainly focused their work on individual cognition to understand the individual knowledge structure and contents, and how individuals perceive, manipulate and interpret information (Levine \& Resnick, 1993; Monteil \& Huguet, 1999). Over the years, the focus of cognitive psychologists has changed. They recognized that social factors are part of cognition, and they also recognized the 
importance of analyzing and understanding the situations in which cognition occurs, thereby leading them to focus on the collective cognition (Levine \& Resnick, 1993).

People do not live alone, and constantly interact with other people in different social environments. In each of those environments, each person has "his or her own intentions and interpretations of the situation, who influence one another's knowledge, opinions, and values and who interact to produce shared cognitive products" (Levine \& Resnick, 1993, p. 587). When people interact and work with each other, they engage in social processes, such as social interaction and communication, which influence cognitive functioning and cognitive outcomes (Levine \& Resnick, 1993; Moscovici, 1989). Thus, cognition is not an individual activity; it is instead a social or collective activity (Levine \& Resnick, 1993). The recognition of importance of collective cognition led to the emergence of team cognition - common knowledge, reasoning processes, and beliefs that team members jointly construct and share (Resnick, 1993; Tindale, Meisenhelder, Dykema-Engblade, \& Hogg, 2001).

Team cognition has its roots in social and cognitive sciences. Social scientists played an important role to the development of this research area. In the beginning of the $20^{\text {th }}$ century, social psychologists, sociologists, and social philosophers, such as Le Bon, McDougall, and Durkheim, argued that the social behaviors were guided by the "collective consciousness" or "group mind" (Tindale et al., 2001, p. 1), which refer to mental states of team members based on their individuals' perceptions, and expectations (Klimoski \& Mohammed, 1994; Tindale et al., 2001). However, besides the importance of shared meaning to understand the social behavior, the psychologists' focus moved from the collective to the individual level. Later, in the end of the $20^{\text {th }}$ century, the interest on the collective resurged (Klimoski \& Mohammed, 1994; Tindale et al., 2001). Theories and research from social psychology and sociology have contributed to the resurgence of interest at the collective level and to the development of team cognition research, such as the symbolic interactionist approach, the social comparison theory, and the theory of social impact (Tindale et al., 2001). In the next paragraphs we describe how those theories influence team cognition research.

The symbolic interactionist approach had an important influence on shared social meanings (Thompson \& Fine, 1999; Tindale et al., 2001). This approach postulates that collective meaning is an important component of social life, and at the same time the social life depends on the shared interpretations people make based on the collective meanings. Moscovici's notion of social representations, which is related to the symbolic interactionist approach, also contributed to the development of team cognition. Moscovici argued that 
groups and teams rely on shared images to make attributions, make causal explanations, and build a common sense (Levine \& Resnick, 1993). This common sense, or shared meaning, is the cognitive context in which team members communicate, coordinate their actions, and is the basis for people interpret and understand the events (Tindale et al., 2001). Thus, team members build and share cognitions based on the socially shared meanings that are developed and modified over time through interaction processes among social actors (Tindale et al, 2001).

Social comparison theory by Festinger postulates that people use the reality that surrounds them to capture the appropriate behaviors and opinions in order to reduce uncertainty, fit in the team or in the organizational context (Tindale et al., 2001). The work of Sherif on social norms also shows that team members use the judgments of others to modify their own judgments (Tindale et al., 2001). Hence, social comparison theory reveals that people in the same team develop similar behaviors and a similar understanding about the reality, or in other words, develop shared cognitions. Latané, with his theory of social impact, showed that people tend to cluster in the social environments based on similarity and, consequently, people tend to become similar to each other (Tindale et al., 2001). Thus, Latané's work showed that shared cognitions result from social interaction and they are a consequence of self-organizing principles that occur in the social environment. Further, Tajfel's theory of social identity proposes that people categorize themselves and others in terms of prototypes (Tindale et al., 2001). Team members distinguish themselves and others based on ingroup prototype (people minimize the differences among team members from the same team) and outgroup prototype (people maximize the differences between team members from their team and from other teams). People in the same team understand what the team characteristics that distinguish them from other teams are, which lead to common attitudes, feelings and behaviors. Thus, team members develop a shared representation of team properties (Tindale et al., 2001).

Team cognition was also influenced by the social decision heuristics or team decisionmaking strategies. In team decision making contexts the majority of the processes occur and lead to a convergence in individual member opinion after the decision, which revealed that team decision strategies can lead to shared opinions in teams (Kameda, Tindale, \& Davis, 2003). This is related to the heuristic function of mental models in decision making. Heuristics refer to mental shortcuts people use when they face difficult and complex tasks to solve problems and make judgments in a quick and efficient way (Simões, 2001; Tversky \& 
Chapter 1

Kahneman, 1973). People use these cognitive shortcuts to simplify the selection and analysis of information without the constant need to stop to think about the next course of action, and not taking into account unnecessary information. Thus, heuristics reduces the cognitive demands and reduces the time spend in making decisions (Simões, 2001; Tversky \& Kahneman, 1973). Heuristics and other decision biases are often used to explain individual decision making because "the information-processing demands of most decisions are far from simple and are often beyond human capacity, thus requiring individuals to simplify the process" (Ilgen, Major, Hollenbeck, \& Sego, 1995, p. 128). As people organize knowledge into structured and meaningful patterns that are stored in memory, people are able to process information in a rapid and flexible way which causes complex cognitive functioning (CannonBowers et al., 1993). When people recover information from memory, they can more easily access to related information. Thus, "mental models provide a heuristic function by allowing information about situations, objects, and environments to be classified and retrieved in terms of their most salient and important features" (Cannon-Bowers et al., 1993, p. 226).

\section{Team-level information processing model}

Like individuals, teams process important and available information in order to perform cognitive and intellectual tasks. However, team information processing is different from individual information processing, as at the team-level information processing occurs within and among the minds of team members (Hinsz et al., 1997). In order to understand how teams collectively process information, share information and produce effective outcomes, Hinsz and colleagues (1997) conceptualized a team-level information processing model based on the individual-level information processing model (Massaro \& Cowan, 1993). Importantly, the information teams process depends on the information available in the contexts where they operate, and on the task they accomplish (Hinsz et al., 1997).

The team-level information processing model by Hinsz and colleagues (1997) comprises eight stages and refers to "the degree to which information, ideas, or cognitive processes are shared, and are being shared, among the group members and how this sharing of information affects both individual- and group-level outcomes" (Hinsz et al., 1997, p. 53). A team that is able to successfully process information is more likely to effectively accomplish their outcomes. However, there are some factors that may affect the way teams process information throughout the eight phases identified by Hinsz and colleagues (1997). Therefore, 
teams that effectively process information are more likely to successfully accomplish their goals.

In the first stage - processing objectives -, teams acquire and process information based on objectives, tasks or collective goals. The objectives that teams process represent the reality that team members share and, as team members may not have a common frame to process the objectives, team members may treat and process the information in different ways. Thus, the way team members treat information (in a similar or different way) affects the other phases of information processing, and in the long term affect team performance. Processing objectives may be affected by a number of team factors that can stimulate or stifle the subsequent stages of the information processing model. For instance, when cues are available in a task that needs to be shared, team members tend to distribute the resources equally; whereas when the cues are absent, they tend to favor themselves distributing the resources in an unequal way (Samuelson \& Allison, 1994; Hinsz et al., 1997). In addition, team members with different backgrounds and/or different demographic characteristics are likely to process information in different ways (Hinsz et al., 1997; Levine \& Resnick, 1993). Finally, team members with different roles in the team may process information differently, and the leader may influence the information that team members process and discuss when he/she state his/her preferences early in a discussion (Hinsz et al., 1997; Janis, 1982)

In the second stage - attention -, team members attend to information in order to process it. In this stage, team members need to face some challenges to ensure that all the team members focus their attention on the information that is supposed to. In fact, teams can be harmful for team member attention focus. As a team has different team members, each of them may focus their attention on the self, and/or may not attend to all the information. This happens because team members may distract each other from the intellectual and cognitive task. Team members need to overcome those distractions and be able to focus their attention on as much information as possible in order to take advantage from their great capacity to attend the information. Another challenge concerns to the distribution of information. Although in some teams there is no need for all team members receive the same information, at least two team members need to receive the same information in order to be a focus of attention in the team. Time pressure, for instance, may affect the way team attend information. When teams are under high levels of time pressure, the teams focus on the task accomplishment; whereas when teams are under moderated levels of time pressure, they focus on the quality of the outcome attending the available information carefully (Hinsz et al., 1997). 
In the third stage - encoding - teams structure and interpret the information. This stage is related to the representation of the information at the team level. The mental representation of information at the team level happens when the different team members focus on the same aspects of the task information, create the same meaning to it, encode this information in their own mental representation, thereby combining the individual mental representations (i.e., the individual mental models) into a shared vision of information (i.e., a shared mental model). However, the integration of the individual mental representation may not lead to a shared mental representation. Team members may focus on different aspects of task information, or they may focus on the same aspects of task information but create different meaning to them. Thus, team members encode the information in their own mental model in different and incompatible ways. Consequently, team members may have different opinions and ideas based on the information they encoded and on their different mental models. Although a shared vision of the information among the team members may be difficult to achieve when team members have different ideas, they need to explicitly clarify them within the team. This clarification may help them to share a mental model (Hinsz et al., 1997).

The fourth stage - storage - plays an important role in the team-level information processing model because team members may store a large amount of the information they need to perform tasks. However, team members do not store all the amount of information they could do because losses may occur due to the effort to remember the information at the team level. To overcome this obstacle, teams may develop strategies to help them to store important information - they may create a transactive memory system (Wegner, 1987). With a transactive memory system, each team member is responsible for process a specific kind of information becoming an expert in that information. Consequently, the team is able to store a large amount of information (Hinsz et al., 1997).

In the fifth stage - retrieval - team members retrieve the required information from storage. The retrieval of information at the team level has a number of advantages over the individual retrieval of information. For instance, team members may recognize that the information retrieved by other team member is incorrect and they may work together to correct it. In addition, if the team retrieves incorrect information, a team member may notice that and force the team members to search for the correct information in their memory. Thus, team members may benefit from strategies to make the retrieval process more efficient and 
increase their storage capacity. For instance, teams may be responsible for remember specific information (Hinsz et al., 1997).

Then, in the processing in the work space stage, teams integrate information based on rules, strategies, and procedures. Like individuals, teams may use heuristics to combine and integrate information, but when they need to make a decision they tend to choose risky alternatives. Nevertheless, teams use rules and strategies in a more reliable and consistent way than individuals because there is less variability in their judgments. An obstacle may occur in this stage that should be overcome - cognitive loafing. As team members know there are other people who can process the information, team members process less information in a less complex and extensive way, because they know that their information will be combined with the information from other members (Hinsz et al., 1997).

All the stages described above influence the response stage. Team responses are related to individual judgment and decision making, and represent the collective intention of the team. Team responses depend on a number of factors, such as, the type of task, and the procedures to make decisions. The type of task may influence "what information is processed and how the information is processed, as well as the type of response generated" (Hinsz et al., 1997, p. 51). Different procedures may also influence the team response. For instance, agendas can control the way teams process information, determine the alternatives that teams consider and determine the sequence in which teams consider the alternatives. Decision rules can also affect the distribution of team members' responses. Teams that need to reach a unanimous decision are more committed to a previous solution than teams that need to achieve a majority decision (Hinsz et al., 1997).

The final stage - feedback - is important for team-level information processing because it can add new information that allows the team to change the situation, the team interaction and the responses. Feedback can be related to team performance, ideas, preferences, and behaviors, and can affect team processes and outcomes. Feedback can also focus on the cognitive processes used by team members, which improve individual learning and satisfaction.

Over the team-level information processing model, learning is an important process for the development of the knowledge structures used by team members. In fact, over time team members learn about "what information can be shared and how sharing changes over time as members learn what other members know" (Hinsz et al., 1997, p. 53). In teams, learning plays an important role for improving performance because through the interaction 
team members learn aspects related to taskwork, such as knowledge and skills needed to accomplish the tasks, and to teamwork, such as interpersonal skills needed to interact with each other (Hinsz et al., 1997; Marks et al., 2001).

In sum, team cognition has its roots in social and cognitive sciences. These roots are crucial to understand how individual team members are able to integrate their own mental models into a shared mental model. Thus, based on the team-level information processing model, a team is more likely to share a mental representation of knowledge (i.e., to share a mental model) when teams treat information and process the objectives in a similar way and have similar background; when they are able to attend a large amount of information; when they are able to encode similar aspects of the task information and create similar meaning to them; and when they are able to store as much information as possible in their minds (Hinsz et al., 1997). A team that accomplishes these steps is more likely to recover the information when it is needed, to combine and integrate information, and in turn to make decisions and accomplish its tasks successfully (Hinsz et al., 1997).

\section{SHARED MENTAL MODELS AND SHARED TEMPORAL COGNITIONS}

Team cognition research has emerged over the last years. Nevertheless, although progress has been made in this area, there are a number of avenues to pursue. In this chapter, I explain what shared mental models and shared temporal cognitions are, I clarify the difference between these two constructs, and I distinguish these two constructs from other cognitive constructs (transactive memory system and team situational awareness). Further, I explain what it is already known about the role of shared mental models and shared temporal cognitions on team functioning. Finally, I describe how this work contributes to team literature and, in particular, to team cognition literature.

\section{Team cognition}

When we work in teams we may realize that sometimes we do not need to communicate all the steps we need to make, ask what we need to do after someone finish a task, who is doing what, or when the deadlines are. When this happens, it is likely we are part 
of a team that has a shared understanding about important aspects of the task. In other words, it is likely that those teams have a shared mental model and/or shared temporal cognition (Cannon-Bowers et al., 1993; Gevers et al., 2006; Klimoski \& Mohammed, 1994). When this happens, it is likely we are engaging in implicit coordination, which occurs when team members are able to coordinate their actions without the need for explicitly communicate, are able to anticipate the need of team members, and to adjust the team members' actions according to the task (Rico, Sánchez-Manzanares, Gil, \& Gibson, 2008).

\section{Shared mental models}

Over the years, a number of researchers has focused on the shared mental model construct, leading to a proliferation of empirical studies in this area (e.g., Edwards, Day, Arthur, \& Bell, 2006; Lim \& Klein, 2006; Mathieu et al., 2000; Resick et al., 2010a, 2010b; Uitdewilligen et al., 2013). Empirical research has systematically shown that when teams develop a shared mental model, team members can anticipate the needs and actions of other members, and adapt their behaviors to fit closely to those of other team members and to task demands (e.g., Cannon-Bowers et al., 1993; Mohammed, Ferzandi, \& Hamilton, 2010). Team members are also able to organize information and establish patterns of response needed to manage the dynamics that occur in their environment, and work in a cooperative and coordinated way (Cannon-Bowers et al., 1993; Salas et al., 2005; Zaccaro et al., 2001). Thus, in order to effectively engage in team processes and accomplish the team goals, team members need to be "on the same page" regarding to what tasks they need to perform, with whom they need to interact and coordinate, and when they need to accomplish the tasks (Cannon-Bowers et al., 1993; Mohammed et al., 2010; Mohammed, Tesler, \& Hamilton, 2012).

\section{Shared mental models dimensions}

Team members may hold multiple mental models, about different domains, over the team lifecycle (Cannon-Bowers et al., 1993; Klimoski \& Mohammed, 1994). Although Cannon-Bowers and colleagues (1993) have identified four content domains - equipment, task, team interaction, and team - Mathieu and colleagues (2000) merged those four content domains into two domains - task and team. These authors argued that the equipment and the task domains reflect the task content domain, and that the team and team interaction reflect 
Chapter 1

the team content domain, which is consistent with the idea that teams need to perform activities related to the task (taskwork) and to work well together as a team (teamwork) (Mathieu et al., 2000). Over the years, researchers have most commonly used and measured task mental models and team mental models. The former refers to a similar understanding about how the task should be accomplished in terms of procedures and practices, as well as about the resources needed to accomplish the task. The later refers to a similar understanding about the team interaction, their responsibilities, the relation between their roles, and the knowledge, skills and abilities of each team member (Cannon-Bowers et al., 1993). Recently, scholars added the temporal mental model dimension referring to team members' understanding about the sequencing of the teams' tasks, deadlines for task accomplishment, and the pacing at which activities should take place (Mohammed et al., 2015).

At specific moments in the team lifecycle teams may only need to use one type of mental model, or may need to use different types at the same time depending on the task they are accomplishing (Cannon-Bowers et al., 1993). However, the different types of mental models may not be developed and may not be mentally accessible at the same time. In fact, the development of one shared mental model dimension may influence the development of other shared mental model dimension (Maynard \& Gilson, 2014). For instance, in some contexts where team members need to work on the task and make decisions from the early beginning of team lifecycle and where deadlines are particularly important for team performance, team members may neglect the interpersonal aspects of teamwork. Therefore, the development of temporal and task mental models may precede and influence the development of team mental models.

\section{Shared mental model similarity and accuracy}

Shared mental models may be similar - a mental model that is similar among team members - and accurate - a mental model that is appropriate for the task according to experts in the respective area (Edwards et al., 2006). Having a similar mental model does not mean that the individual mental models of each team member is identical to each other. In fact, to maximize performance and improve team functioning, the best solution is to ensure that the team members do not have an exact replication of each other's mental models. An exact replication or an extreme similarity among individual mental models may lead to few solutions, 
ideas, and perspectives (Salas et al., 2005; Woehr \& Rentsch, 2003), and may even lead teams to share very cohesive thinking and to suffer from groupthink (Janis, 1972).

Team members that have an accurate mental model have correct knowledge about the best way to accomplish the team task, focus on the right priorities and use appropriate strategies, which helps teams to coordinate their activities and to openly communicate with other team members (Edwards et al., 2006; Marks et al., 2000; Mathieu et al., 2000). In other words, when team members have an accurate mental model, the mental model is an appropriate representation of the task, team, and temporal aspects of the teamwork (Marks et al., 2000).

\section{Shared temporal cognitions}

Shared temporal cognitions refer to "congruent mental representations of the temporal aspects of a specific group task, such as the importance of meeting the deadline, (sub)task completion times, and the appropriate timing and pacing of task activities" (Gevers et al., 2006, p. 54). Team members that share temporal cognitions have similar interpretations, preferences, and expectations about time and deadlines. As team members are able to interpret temporal cues in a similar way, they are able to coordinate their work and to accomplish tasks on time, achieve temporal synchronization, and minimize temporal conflict (Bartel \& Milliken, 2004; Gevers et al., 2006; Standifer et al., 2015).

\section{Shared mental models vs. shared temporal cognitions}

Both shared mental models and shared temporal cognitions refer to implicit coordination mechanisms that help teams to align and integrate their activities into a unified whole (Espinosa, Lerch, \& Kraut, 2004; Ramon et al., 2008; Salas et al., 2005). This means that team members are able to coordinate their tasks and work without the need of directly communicate with each other because they interpret the information and the environment where they work in a similar or compatible way (Salas et al., 2009).

Shared mental models and shared temporal cognitions are cognitive constructs that have similar characteristics. Both constructs refer to a shared understanding among team members about important aspects of teamwork. While shared mental models may be related to task, team, and temporal aspects, shared temporal cognitions are only related to temporal 
aspects of the work. Thus, both constructs may be focused on the temporal issues teams need to deal. A question emerges: What is the difference between a temporal mental model and a shared temporal cognition? While there is no difference in terms of conceptualization (both refer to a shared understanding about the sequencing of the teams' tasks, deadlines for task accomplishment, and the pacing at which activities should take place; Gevers et al., 2006; Mohammed et al., 2015), the difference in terms of operationalization and measurement is substantial (DeChurch \& Mesmer-Magnus, 2010; Gevers et al., 2006; Resick et al., 2010b). We explain in the next sections how shared mental models and shared temporal cognitions are operationalized and measured.

\section{Shared mental model measurement}

Firstly, in order to measure shared mental models, a number of key concepts, aspects, or short sentences that are important to the team task need to be defined based on a detailed task analysis of the team context under investigation (Mohammed \& Hamilton, 2012). Then, shared mental models may be analyzed through different techniques: content (i.e., the focus of mental models), elicitation of content (i.e., the content or components of the mental model), structure representation or structural networks (i.e., the way as the content of mental models is cognitively organized), and representation of emergence (i.e., the representation of the team-mental model as a collection of individuals' mental models) (Mohammed, Klimoski, \& Rentsch, 2000). In order to measure the shared mental models through these techniques, researchers may use different metrics: structural networks, priority rankings, and importance ratings (Mohammed et al., 2000; Resick et al., 2010b). Structural networks refer to the network of relationships among key aspects of the team work associated with the achievement of the team goal. Individual team members evaluate the relatedness between those key aspects. Priority rankings refer to the importance rankings among key aspects or decisions related to the team goal accomplishment. Individual team members evaluate those aspects, ranking them from the highest to the lowest priority for the team goal accomplishment. Finally, importance ratings refer to the importance of key aspects related to the accomplishment of the team goal. Individual team members evaluate the importance of each key aspect without considering other key aspects for the team goal accomplishment (Mohammed et al., 2000; Resick et al., 2010b). As throughout this work shared mental models are mainly measured with structural networks using pairwise ratings - the most generally and considered most valid metric in 
mental models research (Resick et al., 2010b) - in the next paragraph I explain how the structure of shared mental models is analyzed.

To analyze the structure of shared mental models, calculating their similarity and accuracy, a number of network-analysis programs may be used, such as Pathfinder or UCINET (e.g., Lim \& Klein, 2006; Mathieu et al., 2000; Resick et al., 2010b). In this work UCINET is used. This program provides a similarity measure based on Pearson's correlations (Borgatti, Everett, \& Freeman, 2002). In order to calculate shared mental model similarity, a matrix for each team containing the individuals' matrices needs to be done (i.e., the individuals' evaluation of the pairs of sentences; see Figure 1.1). For instance, for a team of three elements, a team-level matrix containing the three individual matrices needs to be done (see Figure 1.1). When different domains of shared mental models are evaluated, different team-level matrices need to be done (i.e., one matrix for each dimension). Further, when shared mental models are evaluated more than one time a matrix needs to be done for each time moment (or more than one for each time, depending on how many dimensions are evaluated). After the construction of the matrices, UCINET calculates the similarity among the pairs of sentences of all team members, for each team-level matrix. This similarity index ranged from -1 (complete disagreement) to 1 (complete agreement/sharedness). The similarity values are also displayed in a matrix. The mental model similarity index of each team is then calculated based on the average of the similarity values. For the matrix displayed in Figure 1.1, the mental model similarity index is 0.84 (i.e., the average between $1 ; 0.756 ; 0.756$ ).
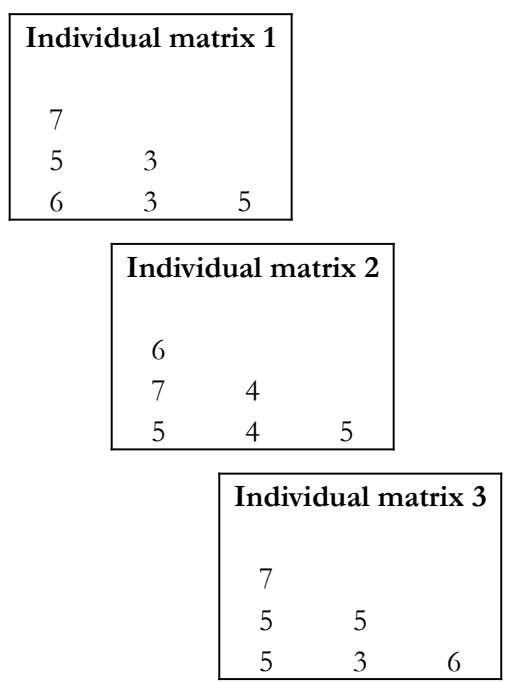

Figure 1.1. Individual-level and team-level matrices.

\section{Team-level matrix}

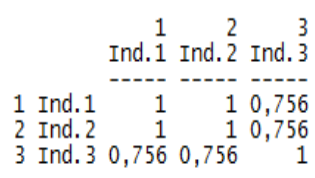


On the left hand-side are presented the individual matrices for a team composed of three elements. On the right hand-side is presented the team level matrix calculated based on the three individual matrices, displayed by UCINET.

In order to calculate shared mental model accuracy, a number of expert-members need to answer the mental models items. Experts could for instance be people that have previously shown high performance on the task or they could be the designers of the task. Shared mental model accuracy can be calculated in a similar way as shared mental model similarity. When accuracy is calculated following this procedure, researchers need to include an individual matrix that contains the average of the individual mental model accuracy scores. Then, accuracy is calculated as the structural similarity between the team members' mental models and those of the experts. An alternative way to calculate shared mental model accuracy may be obtained by calculating the absolute difference between each team member's response and the average response value obtained by the experts for each item (Webber et al., 2000). To calculate the levels of mental model accuracy for each team, the individual team member mental model accuracy scores are averaged. The mental model accuracy index ranges from 0 (completely accurate mental models) to 6 (completely inaccurate mental models). In this work, we followed the second procedure for calculating shared mental model accuracy.

Besides the procedures described above, shared mental models may be assessed through a perceptual approach, in which team members are asked to indicate how similar they perceive the cognitive structures of the team members to be. Johnson and colleagues (2007) developed a measure of 42 items to assess shared mental models in which participants rate the extent to which they agree with each sentence. Sharedness of mental models is calculated through the average (i.e., the average rating for each item, computed for each team) and the standard deviation of the average score which represents "how closely aligned each team member is on any particular item" (Johnson et al., 2007, p. 448).

In sum, different techniques and metrics can be used to measure shared mental models. A number of researchers have suggested that a perceptual approach to measure cognition can be valuable as structural network (Rentsch \& Mot, 2012; Van den Bossche, Gijselaers, Segers, \& Kirschner, 2006). Nevertheless, structural network analysis - although complex and time consuming for participants and researchers - is the only way to capture the organizational structure of knowledge. 


\section{Shared temporal cognitions measurement}

Shared temporal cognitions are evaluated through a four-item scale developed by Gevers and colleagues (2006): (1) In my team/group, we have the same opinions about meeting deadlines; (2) In my team/group, we have similar thoughts about the best way to use our time; (3) In my team/group, we agree on how to allocate the time available; and (4) In my team/group, we have similar ideas about the time it takes to perform certain tasks. As the items are not developed for each context based on a detailed task analysis of the team context, the scale can be used in different contexts more easily than a shared mental model measure. However, with the shared temporal cognition measure it is not possible to capture the structure of the shared knowledge about the temporal aspects of work.

Different techniques may be used to operationalize shared temporal cognitions. One of the techniques consists on the aggregation of the individual scores on shared temporal cognitions scale to the team level (Costa et al., 2013; Gevers, van Eerde, \& Rutte, 2009). The individual scores are aggregated to the team level based on the $r_{\mathrm{wg}(\mathrm{j})}$ values, an estimate of within-group agreement designed for multiple-item scale (James, Demaree, \& Wolf, 1993). The widely-applied cut-off criterion for a good estimate of $r_{\mathrm{wg}(\mathrm{j})}$ refers to a mean value equal or above .70 (James et al., 1993). A different technique, based on a dispersion model (Chan, 1988), consists on the calculation of the coefficient of variation (i.e., the ration of the shared temporal cognition standard deviation to the shared temporal cognition mean; Standifer et al., 2015)

The measurement and operationalization of shared temporal cognition is simpler than that of shared mental models both for participants and for researchers. Nevertheless, the information acquired with these two measures is different. With shared temporal cognitions, it is possible to understand whether a team agrees about the best way to use its time, or to allocate the time available, and whether a team has a strong or weak shared temporal cognition. However, it is not possible to understand the cognitive organization of the temporal knowledge team members have - that is possible with shared mental models measures. So, both shared mental models and shared temporal cognition measures are valuable and important, but the knowledge acquired through them is different. 


\section{The distinction between shared mental models, shared temporal cognition, and transactive memory systems and team situational awareness}

Imagine a medical team that is performing a surgery to the heart and legs of a patient. The surgical team is composed of a cardiac surgeon, an orthopedic surgeon, an anesthetist and nurses. All the team members know the final goal of the surgery -save the patient's life fixing his heart and legs. All the team members have a shared understanding about what needs to be done, what are the necessary equipment and procedures to operate the patient and save his life. They also have a shared understanding about who does what as they understand their role in the task and the role of other surgeons and nurses, as well as who needs specific type of information. For instance, the cardiac surgeon knows that she needs to say to the orthopedic surgeon when her job is done in order to the surgery proceeds to the patient's legs. Further, all the medical team members have a shared understanding about when they need to do a procedure, and when is not safe to do it. They also know the sequence of the tasks that need to be done in order to perform the surgery successfully. This means that the two surgeons, and the nurses, know who operate in this first, and second place. Besides the knowledge shared by the medical team members, they also have specialized knowledge. The cardiac surgeon has skills and abilities related to her specialty, that no one in the team has, that allow her to operate the patient heart. The orthopedic surgeon also has specific knowledge and abilities related to his specialty that allow him to operate the patient legs. Finally, based on the shared mental models that team members have, the surgeons may build a collective understanding about specific information from the patient they are operating. During the surgery something unexpected may occur, for instance, other organs may be affected by a procedure made by one of the surgeons. Thus, the medical team may build a shared understanding at the moment of the surgery perceiving what organ was affected, integrating that information into the surgery procedures that need to be done, and anticipating what will happen because of that unexpected event.

The medical team described above has a shared mental model about the task (what needs to be done), the team (who needs what), and the temporal aspects of the work (when the tasks need to be done). In addition, this medical team has a transactive memory system (Lewis, 2003; Wegner, 1987) (they know who knows what), and team situation awareness (an in the moment understanding of the most important elements of the task and their relation to the team goals) (Endsley, 1995). This means that this team has different forms of team 
cognition that play a different and complementary role in the task accomplishment.

Transactive memory system refers to a cognitive structure that combines the knowledge possessed by each individual team member with a collective awareness of who knows what (Lewis, 2003; Wegner, 1987). This means that each of one team members is responsible for possessing a specific kind of knowledge and consequently the team is able to possess a large amount of knowledge. While shared mental models and shared temporal cognitions are related to different knowledge (task, team, and temporal), transactive memory system is related to team knowledge as it a "team-level mental representation concerning the structure of expertise among the team members or within the team as whole" (Wildman et al., 2012, p. 93).

Like shared mental models and shared temporal cognition, a transactive memory system is an emergent state (Marks et al., 2001). Whereas shared mental models and shared temporal cognition refer to knowledge that is shared by all team members, a transactive memory system refers to knowledge that is distributed across team members. Thus, while shared mental models and shared temporal cognition are composition forms of emergence, transactive memory system is a compilation form of emergence because it comprises a combination of related but distinct lower-level properties that emerge to a higher-level property that is equivalent with its lower level contributions (Kozlowski \& Klein, 2000; Kozlowski \& Chao, 2012b).

Team situation awareness refers to "the team's collective understanding, constructed from the team members' mental models but also incorporating specific information from the current situation" (Uitdewilligen, 2011, p. 174). Team situation awareness is influenced by the knowledge the team already has and emerges over time while teams are performing in a complex and dynamic situation (Cooke, Kiekel, \& Helm, 2001; Uitdewilligen, 2011). Teams recognize cues about the task, team, process, and goals that signal non-routine situations, and incorporate those cues in their shared mental model (Uitdewilligen, 2011; Wildman et al., 2012). Like shared mental models, shared temporal cognitions, and transactive memory systems, team situation awareness is an emergent state (Marks et al., 2001). Nevertheless, whereas shared mental models, shared temporal cognitions, and transactive memory systems are static mental representations of information over some period of time, team situation awareness is a mental representation of information that is dynamic because it changes or evolve constantly and in a rapid way (Wildman et al., 2012). 
Chapter 1

\section{Explored mechanisms between the effect of shared mental models and shared temporal cognitions on team effectiveness}

Shared mental models and shared temporal cognitions work as a cognitive model that helps team members to understand how a task should be performed, how they should work together, and when the tasks should be accomplished. Therefore, those cognitive constructs play an important role in structuring team processes (e.g., communication, coordination, backup behaviours; Mathieu et al., 2000) and team effectiveness (e.g., Burtscher, Kolbe, Wacker, \& Manser, 2011; Edwards et al., 2006; Uitdewilligen et al., 2013).

A number of researchers have analysed the effect of shared mental models on team processes, and on team performance. For instance, Mathieu and colleagues (2000) found that team mental models benefit team performance and that this relationship is mediated by team processes (strategy formation and coordination, cooperation, and communication). Taskmental models benefit team processes, and in turn performance, although they do not have a direct effect on performance. Later, Mathieu, Rapp, Maynard, and Mangos (2010) found that collective efficacy mediates the relationship between task mental models and team effectiveness. Marks and colleagues (2000) also showed the positive effects of shared mental models on team processes and performance. In particular, they found that task mental model similarity, but not accuracy, positively influences communication processes, and that communication processes mediate the relationship of task mental model similarity and accuracy on team performance. Their findings also suggest that the stronger the relationship between task mental model similarity and team communication, the less accurate team mental models are. Further, the more accurate the mental models, the weaker the relationship between mental model similarity and performance is. Later, Marks and colleagues (2002) found that shared mental models improve team performance through coordination and backup processes.

Regarding the effect of shared mental models on team effectiveness, findings have shown that task mental model similarity and accuracy predict team performance (Edwards et al., 2006; Lim \& Klein, 2006), and that task mental model accuracy (but not similarity) mediates the relationship between team ability and performance (Edwards et al., 2006). Burtscher and colleagues (2011) although failed to show a direct effect of task mental model similarity and accuracy on team performance, showed a positive interaction effect between similarity and accuracy on performance. In particular, they found that when accuracy is low, 
similarity is not related to performance, and when accuracy is high, similarity is positively related to performance. Uitdewilligen and colleagues (2013) showed that task mental model updating (i.e., "changing mental models in line with changes in the task situation", p. 129) is positively related to postchange team performance, and that the development of novel team interaction patterns after a structural change mediate the relationship between task mental model updating and postchange team performance. Guchait's (2016) findings suggest that shared mental models influence team engagement and in turn foster team performance and team satisfaction. Further, empirical findings show that shared mental models are positively correlated to client satisfaction and team viability (Rentsch \& Klimoski, 2001), and that whereas task mental model similarity is positively related to team viability, task mental model accuracy is not (Resick et al., 2010a).

Research on shared temporal cognitions is more scarce than research on shared mental models. Empirical studies have mainly showed that shared temporal cognitions promote meeting deadlines (Gevers et al., 2006, 2009) and foster team satisfaction (Gevers \& Petters, 2009; Standifer et al., 2015). Gevers and colleagues (2006) found that shared temporal cognitions facilitate meeting a deadline when teams work early on the task, long before the deadline. Later, Gevers and Petters (2009) found that shared temporal cognitions promote team member satisfaction, and Gevers and colleagues (2009) found that when shared temporal cognitions increase over the team lifecycle teams are more likely to meet a deadline. These authors also found that shared temporal cognitions lead to coordinated action (i.e., "where optimal working relations are established within the team and members execute intended actions in an integrated and timely manner", Gevers et al., 2009, p. 303) and in turn lead the team to meet the deadlines. These authors also found that initial temporal planning promotes high levels of shared temporal cognitions at the beginning of team lifecycle (Gevers et al., 2009).

Mohammed and Nadkarni (2014) conducted a study in an Indian company where they found that shared temporal cognitions attenuate the negative effects of polychronicity diversity (i.e., a preference for engaging in several tasks at the same time; Bluedorn, Kalliath, Strube, \& Martin, 1999) on team performance. Further, they found that shared temporal cognitions foster team performance. Recently, Standifer and colleagues (2015) conducted a multi-cultural study that showed that when teams do not have shared temporal cognitions they engage in temporal conflicts, thereby decreasing team satisfaction. The findings also showed that shared temporal cognitions foster action processes and in turn team satisfaction. Further, 


\section{Chapter 1}

the results showed that teams that have shared temporal cognitions engage in action processes, and are able to diminish the level of temporal conflict which increases team satisfaction.

Those studies suggest that shared mental models and shared temporal cognitions have a positive influence on a number of team processes and in different criteria of team effectiveness. However, although the recent developments in shared mental models and shared temporal cognition research, there are still unanswered questions. For instance, the effect of shared mental models on other important team processes, such as intragroup conflict, team creativity, and team learning, has not yet been investigated. Throughout the team lifecycle, teams interact with each other and they may engage in conflicting situations if they have different points of view about the best way to accomplish the tasks, the delegation of work, the time they have to perform the tasks, or even about personal issues (Jehn, 1995, 1997; Standifer et al., 2015). Therefore, it is important to analyze whether intragroup conflict constrains the positive effect of shared mental models and shared temporal cognitions on team effectiveness. As organizational contexts where teams operate are more and more competitive, teams need to be creative in accomplishing their tasks. Shared mental models may either promote creativity because team members are able to cooperate with each other to develop creative ideas (Burke et al., 2006a), or may constrain creativity because team members do not discuss different ideas (Skilton \& Dooley, 2010). Finally, teams need to learn from each other in order to adapt to recurrent changes in their work environment and to achieve their goals. Shared mental models may facilitate the engagement in team learning behaviors because team members are more likely to coordinate their work and communicate ideas that are aligned with the task demands. The effect of shared mental models on intragroup conflict, team creativity, and team learning deserves attention by researchers.

In addition, team leaders may play an important role in reducing temporal conflict and in promoting team performance, by employing temporal leadership behaviors that help team members to plan their work and manage the time available to accomplish their tasks on time. Nevertheless, shared temporal cognition may substitute for temporal leadership in reducing temporal conflict when leadership is weak or absent. When team members have a shared temporal cognition they may use this common knowledge to help them to regulate the team members behaviors related to temporal issues and to avoid conflicts. The idea that shared temporal cognitions may function as a substitute of temporal leadership for reducing 
temporal conflict in teams needs to be investigated. These unanswered questions will be explored in the next section.

\section{Unexplored mechanisms between the effect of shared mental models and shared temporal cognitions on team effectiveness}

Imagine this situation. John is member of a project team composed of five people, which has as a main objective: to create a commercial for a new car. The team has one month left to complete the project and present the final version to the client. The team has been working together for four months, and the client has been pressuring the team to ensure the commercial will be ready on the agreed upon time. The team is likely to fail this goal. From the beginning, team members do not agree on how to accomplish the task, on what resources the team has, on how to interact with each other, and on how much time they have to accomplish the tasks. Consequently, team members are not able to develop creative ideas for the car's commercial. In addition, team members do not discuss and reflect with each other on the way they have worked. Even the team leader has failed to provide temporal guidance coordinating the team to meet client deadlines, and urging the team to finish the (sub)tasks on time. Conflicts have emerged among team members, which has damaged interpersonal relationship, and team functioning. While the time goes by, this team has no product to deliver to the client, team members are unsatisfied with the way the team has been working and they are desperately waiting for the end of the project to leave this team.

The example above demonstrates the consequences for the team and for the client when teams do not have shared mental models and shared temporal cognitions. Although those problems may reflect the reality of teams performing different tasks in diverse contexts, thus far researchers have not examined whether teams that have shared mental models and shared temporal cognitions are able to minimize conflict, develop creative ideas and learn from each other; or whether team leaders that employ temporal leadership behaviors are able to minimize temporal conflict and facilitate performance. In this section, I discuss these unexplored mechanisms and boundary conditions for the effect of shared mental models and shared temporal cognitions on team effectiveness.

Empirical studies have demonstrated that shared mental models promote team processes and effectiveness (Marks et al., 2000; 2002; Mathieu et al., 2000; 2010; Burtscher et al., 2011). However, it is unknown in which moment of the team lifecycle, shared mental 
Chapter 1

models are more important to both team processes and effectiveness. Researchers have argued that team members need to establish the best strategy to accomplish the tasks, and to interact with each other from the beginning of the team lifecycle (Erickson \& Dyer, 2004; Hackman, 1987; Mathieu \& Rapp, 2009). A solid foundation early in the team lifecycle about the important aspects of teamwork may prevent teams to engage in conflicting situations and promote team effectiveness. However, this aspect has not been examined and deserves researchers' attention.

A small number of researchers have analyzed the effect of shared mental models and shared temporal cognitions on team processes, and in turn on effectiveness (Marks et al., 2000, 2002; Mathieu et al., 2000, 2010). Most of the studies have analyzed their direct effect on team effectiveness (Wildman et al., 2012). In order to use shared mental models and shared temporal cognitions for diagnostic and intervention purposes, it is necessary to clarify what is expected to change when teams share an understanding about relevant aspects of teamwork, and why team effectiveness improves (Cannon-Bowers \& Salas, 2001; Wildman et al., 2012). In the next paragraphs, the effects of shared mental models and shared temporal cognitions on team processes - team creativity, intragroup conflict, and team learning - and on team performance are discussed.

The effect of shared mental models on team creativity is not directly obvious. It could be argued that when team members have similar knowledge structures they are less likely to discuss different points of view and therefore will have less creative ideas (Skilton \& Dooley, 2010). At the same time, it could be argued that shared mental models potentiate creative ideas because a shared understanding about important aspects of the teamwork facilitates effective coordination and cooperation among the team members, which are crucial for team creativity (Burke et al., 2006a). Nevertheless, it is unknown whether shared mental models inhibit creative ideas or whether they potentiate creative ideas.

Intragroup conflict is likely to play a role in the relationship between shared mental models and creativity, as well as in the relationship of shared mental models and shared temporal cognitions on team performance. As a dysfunctional team process, intragroup conflict may hamper the influence of shared mental models on team effectiveness. It is important that team members are able to interact with each other and work without experience tension, and disagreements based on personal issues (relationship conflict), task delegation (process conflict), and time allocation (temporal conflict) because positive interpersonal relations play a crucial role in linking shared mental models with effectiveness 
(Klimoski \& Mohammed, 1994). It is also important that team members are able to engage in discussions about tasks' content and ideas (task conflict) because it may facilitate high quality team decisions and performance (Jehn, 1995; Amason, 1996). Thus far, no empirical study analyzed whether teams that have shared mental models are able to diminish relationship conflict, process conflict and temporal conflict, and improve task conflict, thereby promoting team effectiveness (De Dreu \& Weingart, 2003; de Wit et al., 2012; Jehn, 1995; Passos \& Caetano, 2005; Standifer et al., 2015). Moreover, thus far, it was not studied the effect of shared mental models on intragroup conflict, and in turn on creativity. While the dysfunctional types of conflict may impede team members' ability to develop creative ideas, task conflict may facilitate creativity because teams discuss different ideas and opinions.

As team contexts are increasingly complex and demanding, teams need to adjust their shared mental models and learn from each other to be able to evaluate and analyze situations in order to adapt quickly and appropriately to recurrent changes that may occur (Burke et al., 2006; Rosen et al., 2011; Uitdewilligen et al., 2013). In fact, a number of theoretical models have postulated that shared mental models play a crucial role in promoting team learning processes (Tindale, Smith, Thomas, Filkins, \& Sheffey, 1996; Tindale, Stawiski, \& Jacobs, 2008). However, empirical studies that analyze the relationship of shared mental models on team learning (Guchait \& Hamilton, 2013), and the relationship of team learning on team adaptation and performance are lacking. In particular, it is important understand the interaction effect between shared mental model similarity and accuracy on team learning because teams with similar and inaccurate mental models may not engage in learning behaviors as much as teams with similar and accurate mental models. In addition, there is a need to analyze the immediate effect of team learning processes on performance, and the continuous benefits of team learning over time. Teams that discuss the errors they make, and discuss and update their working methods and strategies are more likely to improve their performance over time. Further, shared mental models may work as boundary conditions to the positive effects of engaging in team learning processes, as shared mental models ensure that teams have a common understanding of the teamwork (Bunderson \& Sutcliffe, 2003a; Edmondson, 2003; Fiol \& Lyles, 1985). Shared mental models may constitute a critical factor that may determine under what conditions team learning processes may be beneficial. Thus, it is important analyze the moderating role of shared mental models between team learning processes and performance improvement. 
Over the last years, organizational contexts in which team operate have become increasingly competitive and teams have to face a number of temporal challenges to integrate and coordinate their work processes. Temporal mental models and shared temporal cognitions are increasingly important as teams need to share an understanding about the time they have to work on the tasks and the moment in which they need to accomplish the final goal. Temporal mental models are particularly important to promote the team learning process because team members make an efficient use of the team's time, thereby creating more time for the team to engage in learning behaviors. Nevertheless, the temporal dimension of shared mental models has been scarcely analyzed (Mohammed et al., 2015). As teams need to face temporal challenges, temporal conflicts may emerge among team members creating tension and dissatisfaction that hinder the teams to perform well. Managing the time available to the team and its members it is crucial in order to avoid temporal conflict (Mohammed \& Nadkarni, 2011; Standifer et al., 2015). However, the mechanisms that team leaders and team members may use to avoid or minimize temporal conflict and to improve team performance are unknown. Two different types of mechanisms may be used to do that: temporal leadership (an explicit coordination mechanism) and shared temporal cognitions (an implicit coordination mechanism). Team leaders, by employing temporal leadership behaviors, may help the team to know the deadlines, prioritize tasks, and to allocate the time to each task. Shared temporal cognitions help team members to anticipate what other team members need and to synchronize and coordinate their actions. Thus, shared temporal cognitions may substitute temporal leadership in reducing temporal conflict and improving team performance. This means that when team members have an implicit way to temporally coordinate, they may not need an explicit way to minimize temporal conflict and improve team performance. The effect of both temporal leadership and shared temporal cognitions on temporal conflict and on performance, as well as the role of shared temporal cognitions in substituting for temporal leadership, deserves the attention of researchers.

\section{Summary}

As teams increasingly perform intellectually challenging and complex tasks, team cognition constitutes a key-concept for understanding successful team task accomplishment. Shared mental models and shared temporal conditions have some similar characteristics their conceptualization - and distinct characteristics - the knowledge they capture. Both 
constructs are valuable and important to understand the role of team cognition on team processes and team effectiveness. This thesis contributes to fill three gaps in the team and team cognition literatures. First, team cognition research has not provided insights about the time moment in the team lifecycle in which shared mental models are most important for team processes and effectiveness. A shared mental model established at the beginning of the team lifecycle may help teams to avoid disruptive interaction, namely relationship conflict, and to achieve their goals. Second, in team cognition research it is yet fully established clear why shared mental models and shared temporal cognitions facilitate team effectiveness. These cognitive constructs may facilitate team effectiveness by promoting healthy interactions among team members (i.e., avoiding conflict situations), creative ideas, and learning experiences. Third, team research has neglected the role time plays as content and as a dynamic process. Regarding time as content, temporal inputs (e.g., temporal mental models, shared temporal cognitions, and temporal leadership) may influence temporal processes (e.g., temporal conflict), and foster team performance. Further, shared temporal cognitions may substitute for temporal leadership in minimizing temporal conflict. Regarding time as a dynamic process, the improvement of team performance over time may be explained by team learning behaviors and shared mental models.

\section{AIM AND OVERVIEW OF THE THESIS}

Shared mental models and shared temporal cognitions play a crucial role in promoting team processes and effectiveness. Although the effect of those two cognitive aspects on team coordination, communication and cooperation has already been investigated (Marks et al., 2000; Mathieu et al., 2000), the effect of shared mental models and shared temporal cognitions on other important team processes in which teams engage has not been investigated. Positive interpersonal relationships are vital to link shared mental models and shared temporal cognitions with team effectiveness. However, team members may engage in conflicts. Further, team members need to develop creative ideas and solutions, and to learn from each other to achieve their goals and survive in increasingly competitive, complex and demanding work environments. Shared mental models may help team members to avoid intragroup conflict, to foster team creativity, and to facilitate the engagement in team learning behaviors. Further, in organizational contexts teams increasingly face temporal challenges in integrating and coordinating their work processes. Although progress has been made on 


\section{Chapter 1}

research regarding the role of time-related aspects in teams, research that analyzes the temporal dimension of shared mental models (Mohammed et al., 2015), as well as, the joint effect of different temporal constructs on team processes and performance (Mohammed \& Nadkarni, 2014) is scarce. In addition, researchers have not analyzed the effect of shared mental models and shared temporal cognitions on team processes and on team effectiveness in different time moments.

The aim of this thesis is threefold. First, I aim to investigate shared mental models and shared temporal cognitions as basic conditions to promote positive relations among team members, to promote creativity, to facilitate the engagement in team learning behaviors, and in turn to foster team effectiveness. Second, I aim to analyze the effect of shared mental models and shared temporal cognitions on a number of functional team processes (task conflict, team creativity, team learning) and dysfunctional team processes (relationship conflict, process conflict, and temporal conflict), and their effects on team effectiveness (team performance, team satisfaction, and team viability). Third, I aim to analyze three time-related constructs by relating them with both shared mental models and shared temporal cognitions, and team performance. In particular, I analyze temporal mental models (examining whether they are a pertinent dimension of shared mental models, and examining their effects on team processes and team performance), temporal leadership, and temporal conflict. Related to this last objective, I aim to analyze the effect of shared mental and shared temporal cognitions on team effectiveness over time in two distinct ways: 1) I analyze shared mental models and shared temporal cognitions, team processes and team effectiveness in different time moments; 2) I analyze the combined effect of shared mental models and team learning behaviors on team processes over time. In order to accomplish these objectives, five empirical studies were developed, using the setting of a five-week strategy and management simulation, collecting data in different years.

Table 1.1. summarizes the research questions and presents the methodology of the studies reported in the chapters of this thesis. 


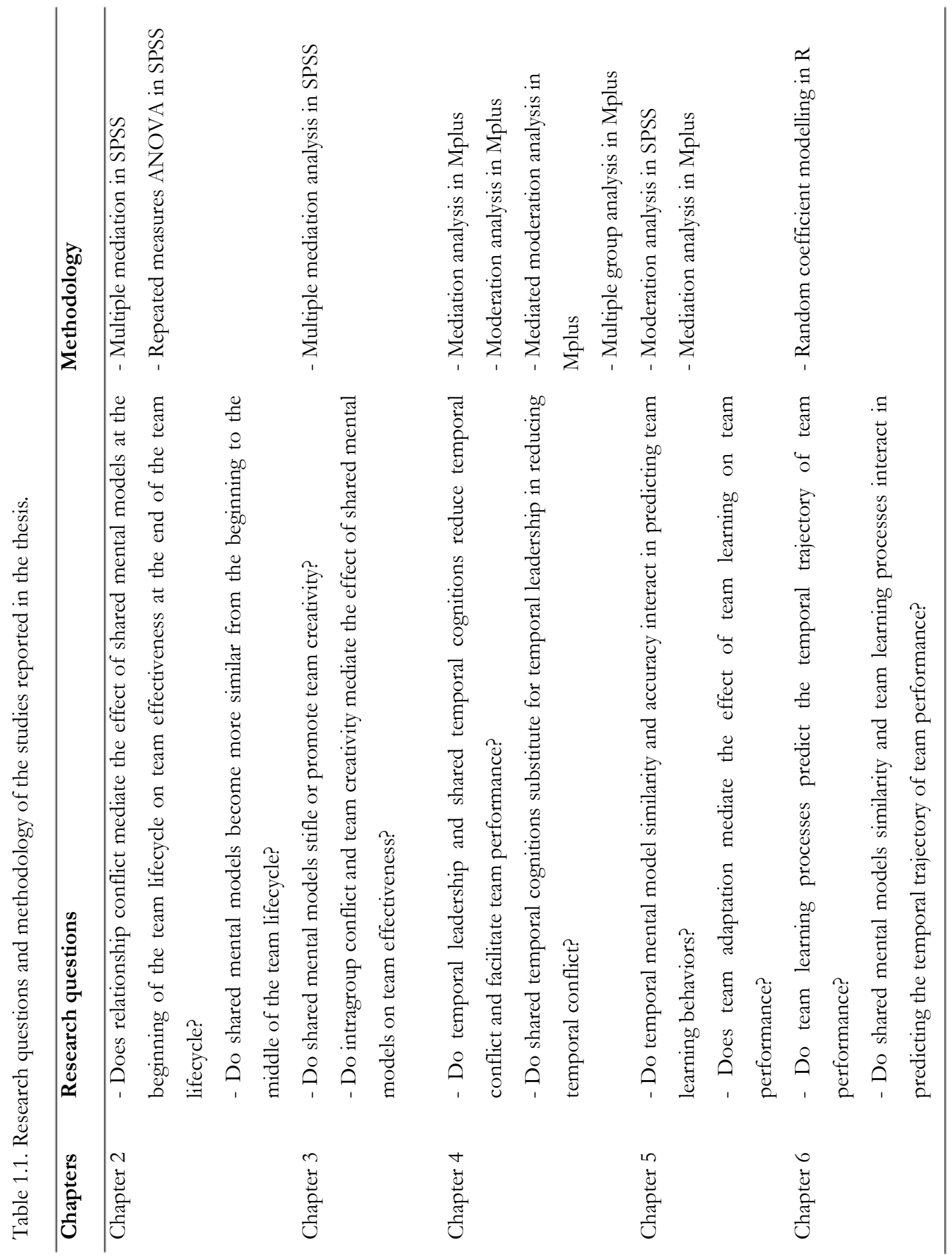


In Chapter 2, we ${ }^{2}$ analyze whether mental model similarity (task and team dimensions) at the beginning of a team's lifecycle influences the level of relationship conflict (as a dysfunctional process) within the team, shared mental model similarity at the middle of the team lifecycle, and in turn team effectiveness (team performance, satisfaction, and viability). Further, we analyze whether shared mental models become more similar from the beginning to the middle of the team lifecycle. We collected data at four different time moments. Findings of the study support the mediating role of relationship conflict between team-mental models (but not task-mental models) similarity at the beginning of team lifecycle and team effectiveness. So, teams that have similar team-mental models experience less relationship conflict and achieve high levels of team effectiveness. The findings also support the mediating role of task-mental models (but not team-mental models) at the middle of the team lifecycle between task-mental models at the beginning of team lifecycle and team effectiveness. Further, findings suggest that shared mental models do not become more similar from the beginning to the middle of team lifecycle.

In Chapter 3, we analyze the mediating mechanisms of intragroup conflict and creativity in the relationship between shared mental models and team effectiveness (team performance and satisfaction). We analyze four types of intragroup conflict: task, relationship, process, and temporal conflict. We collected data at three time points. Findings suggest that the four types of intragroup conflict mediate the relationship between shared mental models and satisfaction, but they do not mediate the relationship between shared mental models and team performance. Creativity positively mediates the relationship between shared mental models and team effectiveness. Further, intragroup conflict and creativity sequentially mediate the relationship between shared mental models and team effectiveness. Importantly, our findings suggest that shared mental models have a positive effect on creativity.

In Chapter 4, we focus on the effects of temporal leadership and shared temporal cognitions on team performance via temporal conflict, and on the power of shared temporal cognitions to substitute for temporal leadership in reducing temporal conflict. We postulate that an implicit coordinating mechanism - shared temporal cognitions - may substitute an explicit coordinating mechanism - temporal leadership - in diminishing conflicts about temporal issues. Findings of the study indicate that temporal conflict mediates the relationship between temporal leadership and team performance, and the relationship between shared

\footnotetext{
2 I use the term "we" to designate myself and the co-authors with whom the various studies were conducted.
} 
temporal cognitions and team performance. Further, findings indicate that shared temporal cognitions function as a substitute of temporal leadership in reducing temporal conflict. This means that when shared temporal cognitions are high, there is no need for temporal leadership in order for teams to experience low levels of temporal conflict.

In Chapter 5, we investigate the moderating effect of temporal mental model accuracy on the relationship between temporal mental model similarity and team learning. Further, we investigate the mediating mechanism of team adaptation in the relationship between team learning and performance. We postulate that when team members have an inaccurate temporal mental model, similar mental models lead them to engage less in team learning behaviors, leading to closed-mindedness (i.e., a phenomenon that occurs when team members engage in collective rationalization ignoring new information and ideas, Dijksterhuis, van Knippenberg, Kruglanski, \& Schape, 1996; Thompson, 2004). We collected data at three time points. Our findings suggest that when temporal mental model accuracy is high, similarity is not significantly related to team learning; while when accuracy is low, the more similar the temporal mental model is, the less team members engage in learning behaviors. Therefore, shared mental models can lead to closed-mindedness when the knowledge the team members share is incorrect. Moreover, our findings suggest that team adaptation mediate the relationship between team learning and performance.

In Chapter 6, we investigate longitudinally whether team learning processes lead to performance improvement, integrating a team process and a learning curve perspective on team learning. Further, we analyze whether the relationship between team learning processes and performance improvement is moderated by the similarity of team members' task, team, and temporal mental models. We collected data at five time points. Our findings suggest that, although team learning behaviors do not have a direct effect on team performance improvement, temporal and task mental models are crucial for the translation of team learning behaviors into performance improvement. In particular, our findings indicate that while when teams have similar task and temporal mental models, engaging in team learning processes is beneficial; when teams have dissimilar task and temporal mental models, engaging in team learning processes is detrimental to performance improvement. Our findings failed to show a significant effect for the moderating role of team mental model similarity.

In Chapter 7, I discuss theoretical and practical implications of the studies reported in this thesis. I present the limitations of the studies, and provide suggestions for future research. 


\section{SIMULATION AND DATA COLLECTION}

The data used in the studies reported in this thesis were collected in a strategy and management simulation - Global Management Challenge ${ }^{\circledR}$ - developed in 1980 by a Portuguese company specialized in developing business simulations. Global Management Challenge ${ }^{\circledR}$ is the largest strategy and management simulation in the world. At this moment Global Management Challenge ${ }^{\circledR}$ is present in more than 30 countries. Importantly, over the years this simulation has been constantly updated. Global Management Challenge ${ }^{\circledR}$ comprises four stages: first round, second round, national final (these first three rounds are run in each competing country), and international final. Data were collected in Portugal, over the last years, in the first round of the simulation that takes place across five consecutive weeks. The five weeks simulate one year and a quarter, with each week simulating three months. In each quarter, teams make decisions on the marketing, production, personnel, purchasing and finance of their fictional company. The main objective of teams in this simulation is to obtain the highest share price in the simulation stock exchange.

The Global Management Challenge ${ }^{\circledR}$ participants need to apply for take part in the simulation and they are free to assemble their own team. Each team has a leader chosen by the team members when they enroll for the simulation. Before the beginning of the simulation, teams receive a manual that explains how the simulation works, and a management report about the companies they will run. Both the manual and the management report can be used during the simulation. Approximately one month before the beginning of the simulation, the teams enroll in two training sessions to be familiarized with the simulation. Teams are organized into groups (each group with a maximum of 8 teams). Each group comprises a competitive market, in which the teams had to compete with one another in a common business environment (the "group") to achieve the highest share price.

A simulator analyzes and compares the decisions made by the competing teams, and calculates the share price of each enterprise and the ranking of all teams after each team made their decision. Then each team receives feedback about their decisions through a management report the simulator produces showing the detailed results in financial and operational terms.

Every year, before the beginning of the simulation, participants were informed by the company responsible for the simulation that a group of researchers would collect data through questionnaires. Participants might choose participate or not. Team members answered online 
questionnaires through a link sent to the members by e-mail at the different time moments. Participants responded to the questionnaires before receiving feedback about their decisions.

Diverse samples, collected in different years of the simulation, were used for the studies reported in this thesis, with the exception of the studies reported in Chapters 5 and 6 in which the same sample was used. 

ChAPTER $2^{3}$

\section{TEAM MENTAL MODELS, RELATIONSHIP CONFLICT AND EFFECTIVENESS}

OVER TIME

${ }^{3}$ This chapter is published as:

Santos, C. M., \& Passos, A.M. (2013). Team mental models, relationship conflict and effectiveness over time. Team Performance Management, 19, 363 - 385. DOI: 10.1108/TPM-01-2013-0003.

http://www.emeraldinsight.com/doi/full/10.1108/TPM-01-2013-0003

This work received the award of the outstanding paper of 2013 published in Team Performance Management. 
Chapter 2

\begin{abstract}
This study aims to evaluate the extent to which similar team mental models (TMMs) at the beginning of a team's lifecycle influence the level of relationship conflict within the team, TMM-similarity at the middle of the team lifecycle, and in turn team effectiveness. Thus far, no research has analysed the mediating role of a dysfunctional team process between TMMsimilarity and effectiveness. The study was conducted in a strategy and management competition involving 414 individuals who comprised 92 teams (3-5 members). We collected data at four moments in time. The questionnaires were developed based on validated scales and adapted for the specific context. Our results provide support for the mediating role of conflict between the similarity of team-TMMs at the beginning of team lifecycle and effectiveness. Our results also provide support for the mediating role of task-TMMs in the middle of team lifecycle between task-TMMs at the beginning of team lifecycle and effectiveness. Findings suggest that teams with more similar TMMs, experience less relationship conflict which in turn improves effectiveness. In this study TMM-accuracy was not analysed. Future research should analyse the role of TMM similarity and accuracy. Further, future research should explore the optimal level of TMM-similarity and when the similarity of TMM is disruptive to teams. This paper sheds light on the role of conflict as a dysfunctional team process between TMM-similarity and effectiveness. Moreover, this paper shows that more research on TMM evolution is needed.
\end{abstract}


Team mental models, relationship conflict and effectiveness over time

\section{INTRODUCTION}

Many authors have tried to identify the key-aspects that distinguish effective from ineffective teams (e.g., Hackman, 1987; Kozlowski \& Ilgen, 2006). Interest in team cognition, particularly in team mental models (TMMs), began to emerge at theoretical and practical levels at the end of the 20th century (Klimoski \& Mohammed, 1994). The mental model construct refers to cognitive representations of knowledge that individuals form regarding the way they interact with their environment (Mathieu et al., 2000; Resick et al., 2010b). TMMs refer to an organized understanding and a mental representation of the knowledge team members share concerning relevant task and team aspects and the environment in which they operate (Klimoski \& Mohammed, 1994). The notion of the TMM refers to the similarity among the mental models of the individual team members (Edwards et al., 2006). The results of initial investigations demonstrated the relevance of TMMs for team effectiveness (e.g., CannonBowers et al., 1993; Orasanu \& Salas, 1993), thus leading to a proliferation of empirical studies in this area.

Despite the recent developments in team cognition, and more specifically in TMM literature, there are still many unanswered questions. Several authors concluded that the influence of TMMs on team outcomes is mediated by team processes, namely communication (Marks et al., 2000), coordination (Minionis, Zaccaro, \& Perez, 1995), and backup behaviours (Marks et al., 2002). Thus far, researchers have only analysed the mediating mechanism of functional team processes, such as communication, coordination, and cooperation, which help team members to improve their work and the interaction among members. However, apart from functional processes, dysfunctional processes may occur during team lifecycle, such as team conflict. As an emergent state, TMM takes time to develop and can change over time, depending on how members collectively interact (Cronin et al., 2011). The existence of dysfunctional team processes, for instance relationship conflict, during team task episodes may constrain both TMM evolution and its impact on team effectiveness (De Dreu \& Weingart, 2003; de Wit et al., 2012; Jehn, 1995). Empirical research has systematically demonstrated that relationship conflict has detrimental effects on team satisfaction and performance (e.g., De Dreu \& Weingart, 2003; de Wit et al., 2012; Lau \& Cobb, 2010). However, no empirical research analysed if TMM-similarity diminishes team relationship conflict, and leads to high levels of effectiveness. 
Finally, TMMs are often measured only at one single point in time (Zhou \& Wang, 2010), which does not allow us to understand how TMMs evolve in on-going task performance episodes (Mohammed et al., 2010; Roe, 2008). If TMMs are measured only at a single point in time it is not possible to know if TMMs increase or decrease from one task episode to another. In addition, it is not possible to know in which moment of the team lifecycle the TMMs are more important to team processes and effectiveness (Mitchell \& James, 2001). Thus, it is necessary to study TMMs over time.

To address these unexplored questions, we investigated the evolution of TMMsimilarity over two task episodes and the influence of TMM-similarity on team effectiveness. Further, we analysed whether relationship conflict is a mediating process between TMMsimilarity and team effectiveness.

\section{THEORETICAL BACKGROUND AND HYPOTHESES}

\section{Team mental models}

The construct of TMM was introduced by Cannon-Bowers and Salas in 1990 as "a way to capture the implicit coordination frequently observed in effective teams and to further understand how teams operate in contexts that are complex, dynamic, and uncertain" (Mohammed et al., 2010, p. 1). TMM literature postulates that when team members share an organized understanding about key elements of the task and the functioning of the team, they can anticipate the needs and actions of other members, and adapt their behaviours to fit closely to those of the other members and to the task demands (Cannon-Bowers et al., 1993; DeChurch \&Mesmer-Magnus, 2010). In fact, TMM works as a cognitive model that helps members understand how a task should be performed and how they should work together with the other team members.

A number of different domains of TMMs have been introduced in the literature (Klimoski \& Mohammed, 1994). Most commonly a distinction has been made between TMMs related to the task and TMMs related to the team. Task-TMMs refer to the common schema team members have about work objectives, team resources, and task duties. Team-TMMs refer to mental representation concerning interpersonal interaction, and team members' skills (Mohammed et al., 2010). Based on wide support for this distinction between the team and the task aspects of TMMs (e.g., Lim \& Klein, 2006; Mathieu et al., 2000; Mathieu, Heffner, 
Goodwin, Cannon-Bowers, \& Salas 2005; Mathieu et al., 2010) we also apply this distinction in the present study.

Thus far, TMMs have been studied mainly at one single point in time and limited attention has been given to the role of time and development in the empirical research on TMM (Zhou \& Wang, 2010). Hence, we still know little about how TMMs evolve in on-going team performance episodes (Roe, 2008). TMMs have generally been conceptualized as emergent states, which are dynamic and changeable (Ilgen et al., 2005; Mathieu et al., 2000; 2008b). Emergent states are dynamic characteristics of a team that vary and evolve over time in reaction to changes to the team context, inputs, processes and outcomes, and that impact team performance (Ilgen et al., 2005; Marks et al., 2001; Mathieu et al., 2008b). Emergent states are distinct from team processes. Team processes refer to “members' interdependent acts that convert inputs to outcomes through cognitive, verbal, and behavioral activities directed toward organizing taskwork to achieve collective goals" (Marks et al., 2001, p. 357). Team processes represent team interaction or team actions that lead teams to achieve their outcomes. In contrast, emergent states result from team experiences, become new inputs to succeeding processes and outcomes, and do not represent team interaction or team actions (Marks et al., 2001).

Several researchers postulate that TMMs become more similar over time, because team members evolve, share, and acquire information, knowledge, and experience with their task and with each other (Levesque, Wilson, \& Wholey, 2001; Mathieu et al., 2000; Thompson, 2004). Over the team lifecycle, team members interact with each other, which leads the team development. Team members become more similar, and build routines that help them to work and achieve high levels of effectiveness (Arrow \& McGrath, 1995; McGrath et al., 2000). From the initial stages of the team lifecycle team members engage in interpersonal interactions through which they share information regarding their roles, capabilities, knowledge and skills (Pearsall, Ellis, \& Bell, 2010). These behaviours lead to TMMs development which in turn leads to a deeper understanding about the roles, responsibilities of each other, and the patterns of behaviour and interaction among team members (Kozlowski \& Ilgen, 2006; Pearsall et al., 2010). Over time team members share information and acquire knowledge that will foster the emergence of TMMs, and through team interaction TMMs become more and more similar (Kozlowski \& Ilgen, 2006; Pearsall et al., 2010). Thus, we expect that TMMs will become more similar over time as team members share and acquire information, knowledge, and experience over the team lifecycle. 
Hypothesis 1 - TMMs become more similar over time.

\section{Team mental models and team effectiveness}

In recent years, team researchers have accepted a complex view of team effectiveness, which considers how teams perform over time as the members continually update their work and way of interaction through team-task episodes (Ilgen et al., 2005; Marks et al., 2001). Several authors have updated the classic model of Input-Process-Output (I-P-O; McGrath, 1964) in order to be adequate for studying teams defined as dynamic, complex, and adaptive entities (e.g., Burke et al., 2006; Marks et al., 2001; Rosen et al., 2011; Salas et al., 2007). Recent models of team effectiveness emphasize the importance of the time dimension to understand team functioning and effectiveness (e.g., Ilgen et al., 2005; Marks et al., 2001). For instance, Ilgen and colleagues (2005) proposed an input-mediator-output-input (IMOI) model in which a final output functions as a new input and consequently gives rise to a new team performance episode. Besides team processes, there are important mediational variables, such as emergent states, that explain the variability in team performance. This model also states that the causal linkages between inputs, mediators, outputs, and new inputs may be nonlinear or conditional (Ilgen et al., 2005).

Empirical studies suggest that TMM-similarity impacts team effectiveness via team processes. Mathieu and colleagues (2000) demonstrated that TMM-similarity has a positive effect on communication, strategy, and cooperation, and consequently on performance. Two studies of Marks and colleagues showed a positive impact of TMM-similarity on communication (Marks et al., 2000), coordination and backup behaviours (Marks et al., 2002), as well as on performance. Therefore, we propose that the TMM-similarity at the beginning of the team lifecycle plays a role on subsequent team processes and effectiveness.

Based on work of Ericksen and Dyere (2004), Mathieu and Rapp (2009) and Wood, Michaelides, and Thomson (2011) we primarily focus on the role of TMM-similarity at the beginning of the team lifecycle. From the early stages, teams need to establish a solid foundation to prevent them from process losses, help them to minimize dysfunctional interpersonal processes (Mathieu \& Rapp, 2009), and promote positive team experiences (Ericksen \& Dyere, 2004). Furthermore, a solid foundation may generate "powerful assumptions within groups about how they will go about their task, work together and 
Team mental models, relationship conflict and effectiveness over time

evaluate their work" (Wood et al., 2011, p. 401). And, in turn, teams are more likely to achieve high levels of performance (Mathieu \& Rapp, 2009). Thus, we argue that TMM-similarity at the beginning of the team lifecycle plays an important role on team processes and on effectiveness.

Based on the theoretical work of Hackman (1987) we distinguish between three main criteria of team effectiveness: performance, viability, and satisfaction. Performance is not sufficient to analyse team effectiveness. It is also important to analyse if the task performance episode contribute to satisfaction and well-being of team members, as well as for their willingness to continue work together (Hackman, 1987; McGrath, 1964). Thus, it is expected that teams with more similar TMMs achieve higher levels of performance (e.g., Marks et al., 2002; Mathieu et al., 2000), are more willing to work together in the future (e.g., Resick et al., 2010b), and feel that the team work experience contributes to their overall well-being and growth (Rentsch \& Klimoski, 2001).

Hypothesis 2 - TMM-similarity at the beginning of the team lifecycle is positively related to (a) performance, (b) satisfaction, and (c) viability.

While TMM-similarity at the beginning of the team lifecycle is important to minimize dysfunctional processes, to promote positive team experiences, and to foster team performance (Ericksen \& Dyere, 2004; Mathieu \& Rapp, 2009), we argue that TMM-similarity in the middle of the team lifecycle is more directly related to team effectiveness. Gersick (1988) showed that team activities at the midpoint are particularly crucial for team effectiveness. At the middle of the lifecycle, teams know clearly how to use their information and knowledge, and they already revised their understanding about the task and team aspects of the work (Gersick, 1988). This means that, at the midpoint, teams start focusing on performing the task. Therefore, TMM-similarity at the middle of the team lifecycle is important for team effectiveness. At this point in time, TMMs are already developed and teams are more likely to have reached the highest possible level of TMM-similarity that leads them to achieve levels of performance. In addition, the TMM-similarity at the beginning of the team lifecycle is likely to lead to TMM-similarity in the middle of the team lifecycle, and in turn to higher levels of effectiveness. Thus, it is expected that: 
Hypothesis $3-T M M$-similarity in the middle of the team lifecycle mediates the relationship between TMM-similarity at the beginning of the team lifecycle and (a) performance, (b) satisfaction, and (c) viability.

\section{Team mental models and relationship conflict}

Intragroup conflict is probably one of the most relevant challenges to team work effectiveness. Conflict is a team process that has often been associated with the internal dynamics of teams (Desivilya, Somech, \& Lidgoster, 2010). As a team process, conflict refers to the team members' interactions directly related to task performance and achievement (Mathieu et al., 2008b). Three different types of intragroup conflict have been identified in the literature: task, relationship, and process conflict (Jehn, 1995; 1997). In accordance with previous studies (e.g., De Dreu \& Van Vianen, 2001; Lau \& Cobb, 2010; Rau, 2005), we only focus on relationship conflict - disagreements based on personal and social issues that are not related to work (Jehn, 1995; Thompson, 2004). We focus on relationship conflict because previous research suggests it is the most disruptive type of conflict, and the conflict type that is most dysfunctional to other team processes (e.g., De Dreu \& Weingart, 2003; de Wit et al., 2012; Mathieu et al., 2008b).

Empirical results systematically show that relationship conflict is disruptive to effectiveness because it implies that members will spend time and energy focusing on personal antagonism, instead of task accomplishment (Simons \& Peterson, 2000). This limits team members' ability to share, exchange, and process relevant information, which can have a negative effect on team effectiveness. Moreover, the anxiety and tension produced by relationship conflict may reduce the team's cognitive functioning, which in turn could lead to a decrease in team performance, diminished satisfaction (de Wit et al., 2012; Jehn \& Mannix, 2001), and viability (Bayazit \& Mannix, 2003). Finally, Rau (2005) posited that team members that experience relationship conflict may neglect or distrust the information provided by other members. Thus, the development and sharing of a common understanding about relevant task and team aspects may be threatened.

TMM literature has postulated that TMM-similarity has a positive impact on team effectiveness via functional processes, such as communication (Marks et al., 2000) and cooperation (Mathieu et al., 2000). However, during team performance episodes some dysfunctional processes occur, such as relationship conflict, which diminishes the interaction among team members as well as their ability to effectively process information (e.g., Rau, 
Team mental models, relationship conflict and effectiveness over time

2005; Simons \& Peterson, 2000). Positive interpersonal relations play a crucial role in linking TMMs with effectiveness (Klimoski \& Mohammed, 1994), thus it is important that teams are able to interact and work without experience tension, and disagreements based on personal issues.

To prevent negative conflicts, it is important that team members establish and understand, from the beginning of task interaction, the tasks goals and procedures, as well as the roles and responsibilities of each other (Pazos, 2012). Only if this is the case, team members are focused on task accomplishment. Team members that share knowledge regarding relevant task and team aspects are more focused on task accomplishment and they discuss aspects related to the task and team interaction that really matter for effectiveness. Consequently, teams that have similar TMMs avoid disagreements based on personal and social issues, and develop the potential to reduce personal antagonism, which means that they experience less relationship conflict (henceforth called "conflict"). Hence, TMMs, as emergent states, influence conflict, as a team process, which is in accordance with the recurring phase model of Marks and colleagues (2001). Therefore, we expected that conflict serves as a mediator between TMMs and effectiveness:

Hypothesis 4 - Conflict mediates the relationship between TMM-similarity at the beginning of the team lifecycle and (a) performance, (b) satisfaction, and (c) viability.

A recent meta-analysis showed that relationship conflict negatively affects emergent states (as proximal team outcomes), such as, trust and cohesion - the two emergent states analysed, so far, in conflict literature (de Wit et al., 2012). Hence, conflict may influence TMMs in subsequent episodes. Team members who experience relationship conflict "are more likely to have different belief structures, understandings, and priorities" (Ensley, Pearson, \& Amason, 2002, p. 370). Team members who do not trust in each other, and who feel tension and friction are less disposed to interact, share information, and discuss ideas (Ensley et al., 2002; Jehn, 1995), which is crucial to TMM development. In contrast, teams that do not experience tension or disagreements based on personal issues are more likely to spend their time and energy exchanging information, discussing about the task and team aspects of work (Simons \& Peterson, 2000). Given that communication and interaction are needed for teams maintain and improve a shared understanding of the task (e.g., CannonBowers et al., 1993; Gurtner, Tschan, Semmer, \& Nägele, 2007; Stout, Cannon-Bowers, Salas, 
Chapter 2

\& Milanovich, 1999), conflict is likely to mediate the relationship between TMM-similarity at the beginning and TMM-similarity at the middle of the lifecycle.

Marks and colleagues (2001) state that emergent states influence team processes, which in turn are likely to alter subsequent emergent states. They add that "this cyclical pattern continues until teams reach more distal team outcomes" (Marks et al., 2001, p. 358). Therefore, we assume that TMMs at the beginning of team lifecycle influence conflict, which in turn influence TMMs at the middle of lifecycle, and finally influence team effectiveness. Although we do expect a relationship between TMMs at the beginning of the team lifecycle, conflict, and effectiveness; and a relationship between TMMs at the beginning of the team lifecycle, TMMs in the middle of team lifecycle, and effectiveness; we also expect a relationship between TMMs at the beginning of the team lifecycle, conflict, TMMs in the middle of team lifecycle and effectiveness. In short, we expect a jointly mediating role of a team process and an emergent state:

Hypothesis 5 - Both conflict and TMM-similarity in the middle of team lifecycle partially and sequentially mediate the relationship between TMM-similarity at the beginning of team lifecycle and (a) performance, (b) satisfaction, and (c) viability.

The research model is represented in Figure 2.1.

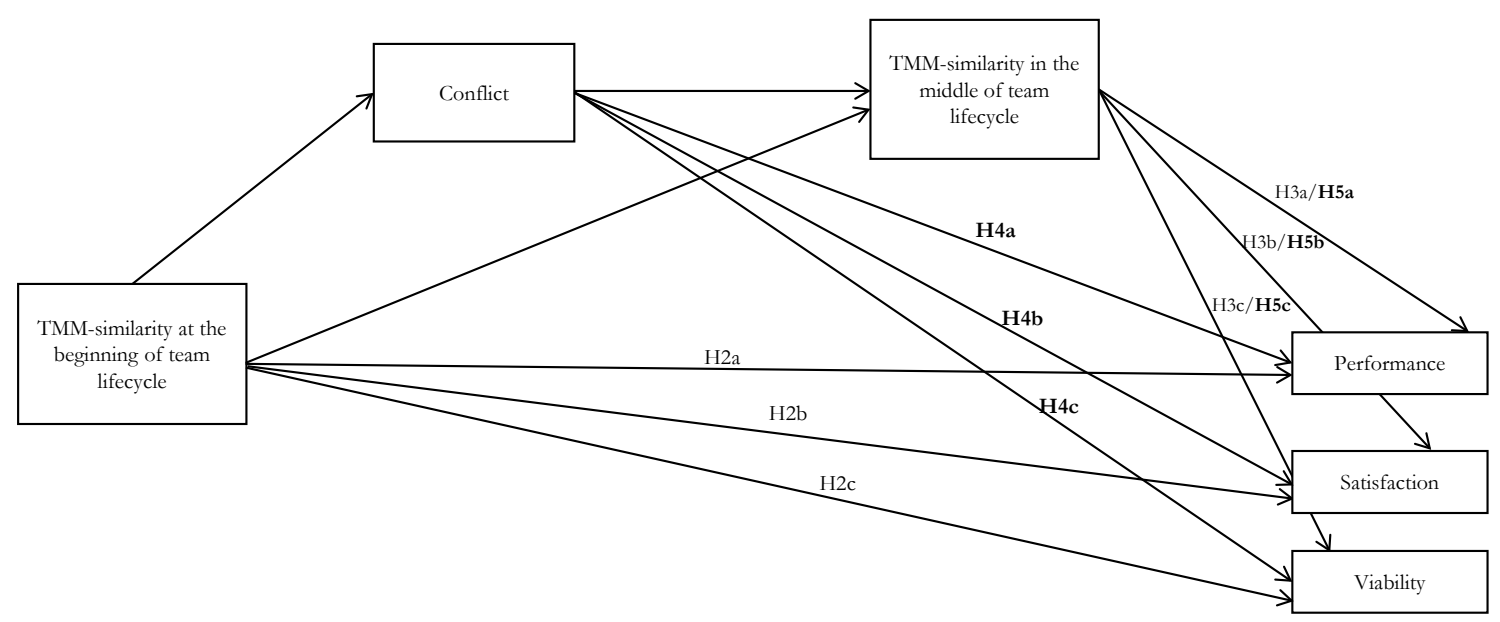

Figure 2.1. Hypothesized main and mediating relationships in this study

Hypotheses in bold include the mediators 
Team mental models, relationship conflict and effectiveness over time

\section{METHOD}

\section{Participants}

A total of 92 teams (414 individuals) participated in this study. Given the relatively large sample size, compared to other TMM studies (e.g., Lim \& Klein, 2006; Marks et al., 2002; Mathieu et al., 2005), we may test our model with a certain amount of confidence. All teams were enrolled in a strategy and management simulation for a 5-week period. The teams were composed of university students (45.7\%), company managers $(40.2 \%)$, or both $(14.1 \%)$. The teams consisted of three to five persons with an average team size of 4.64 persons (s.d. = 0.67). The average age of the members was 28 years $($ s.d. $=8.45)$ and 67.6 percent of the members was male.

\section{Procedure}

Data were collected from the participants of the Global Management Challenge ${ }^{\circledR}$ developed by a company that specializes in developing business simulations (http://www.worldgmc.com). The Global Management Challenge ${ }^{\circledR}$ consists of a management simulation in which each team runs a fictitious Company, with the objective of getting the highest company share price on the simulated Stock exchange. The simulation has been running for more than thirty years, and many top companies encourage their employees to take part in it. The participants choose their own teammates for the simulation.

Teams were organized into groups (each group with a maximum of 8 teams). Each group comprised a competitive Market, in which the teams had to compete with one another in a common business environment (the "group") to achieve the highest share price. The simulation takes five weeks, and each week simulates one quarter of year. Teams managed the company by making decisions about marketing, production, personnel, purchasing, and finance subjects. During the competition, teams received feedback about their decisions. A simulator analysed and compared the decisions made by the various competing teams, and calculated the share price of each enterprise and the ranking of all teams after each team made their decision. Then, the simulator produced a management report for each team, showing the detailed results in financial terms.

Team members answered four different on-line questionnaires. The link to the questionnaires was sent to the members by e-mail at different times of the simulation, and participants answered before having feedback about their decisions. 
Chapter 2

\section{Measures}

TMMs. TMMs operationalization was based on content and similarity ratings. We used the procedure that is used most generally and considered most valid in TMM research (DeChurch \& Mesmer-Magnus, 2010; Resick et al., 2010b). Consistent with Lim and Klein (2006) we asked participants to evaluate the relatedness of sentences, rather than concepts. The sentences describe team procedures, behaviours, and tasks related to each dimension.

We distinguished the two content domains of TMMs: task and team. To operationalize the task-TMMs we adapted three items of the scale developed to assess the Marks and colleagues (2001) taxonomy of team processes (LePine, Piccolo, Jackson, Mathieu, \& Saul, 2008). For team-TMMs, we used three items of the Lim and Klein (2006) scale. Following Lim and Klein (2006), we also included the sentence "My team is extremely effective" for each dimension, in order to analyse the team members' understanding of the relationship between TMMs and effectiveness. We asked each team member to rate the relatedness of the 12 pairs of statements on a 7 -point scale $(1=$ the sentences are not related and 7 $=$ the sentences are extremely related). A list of pairs of sentences is provided in Appendix A.

We selected the three items of the taxonomy of team processes (Marks et al., 2001) that most closely fit with the task performance and the three items of Lim and Klein (2006) scale that better fit with team interaction. The six pairs of sentences for each dimension were discussed and constructed on the basis of recommendations of the company managers who develop the simulation. We assessed TMMs through the taxonomy of team processes of Marks and colleagues (2001), which was developed based on their model of team performance episodes, because it is directly related to the Global Management Challenge ${ }^{\circledR}$. In this simulation teams need to perform several tasks over five temporal cycles. Therefore, team performance happens in a sequence of I-P-O episodes that occur sequentially. A meta-analysis showed that this taxonomy of team processes is positively associated with team effectiveness (LePine et al., 2008). And since conflict refers to a team process (Mathieu et al., 2008b), and conflict management is one of the dimensions of the taxonomy (Marks et al., 2001), we assume that the taxonomy is related to team conflict.

To evaluate TMM similarity we used UCINET (Borgatti, Everett, \& Freeman, 1992), following the procedure developed by Mathieu and colleagues (2000). This network-analysis program provides a similarity measure based on Pearson's correlations. As each member evaluated six pairs of sentences for each TMMs dimension, the first step was to make teammatrices for each dimension at each moment of data collection. Next, we used UCINET to 
Team mental models, relationship conflict and effectiveness over time

calculate the team similarity index for each matrix, which ranged from -1 (complete disagreement) to 1 (complete agreement/sharedness). Similarity values were also displayed in a matrix. The TMM similarity for each dimension of each team was then calculated based on the average of the similarity index for each dimension. TMMs were measured at time 1 and 3.

Relationship conflict. Conflict was measured with four items of Jehn's Intragroup Conflict Scale (1995). On a 7 -point scale $(1=$ never, $7=$ always $)$, the participants indicated how often each behaviour occurred in their team (e.g., "How often is there friction among members in your team"). Conflict was measured in time 2. Exploratory factor analysis revealed only one factor with $77.47 \%$ of variance explained. The four-item scale showed high reliability $(\alpha=$ $.90)$, which is consistent with previous studies (e.g., Jehn, 1995; Jehn \& Mannix, 2001).

Performance. Performance was operationalized through the share price at the end of the simulation. Share price was given in Euros and was automatically calculated by the simulation. The simulation also automatically calculated the team position in their group ranging from first to eighth. We operationalized performance by recoding the share price on 1 to 8 pointsscale through the percentile values - we asked for the cut points for eight equal groups. We created a new variable based on the values of the eight groups, which was used in mediation analyses. The lowest share prices correspond to lower values and the highest share prices correspond to higher values. Our performance measure was discussed and constructed on the basis of recommendations of the developers of the Global Management Challenge ${ }^{\circledR}$.

Satisfaction. One item from the Job Satisfaction Scale (Spector, 1997) was used to measure satisfaction. On a 7 -point scale $(1=$ very dissatisfied, $7=$ very satisfied $)$, the participants indicated how much they were satisfied with all the aspects of their participation in the team. Satisfaction was measured at time 5 .

Team viability. Three items from the study by Bayazit and Mannix (2003) were used. On a 7-point scale $(1=$ totally disagree, $7=$ totally agree $)$, the participants rated the extent to which they agree with each sentence (e.g., "This team could work well together for future projects"). Viability was measured in time 5. Exploratory factor analysis revealed only one factor with $83.98 \%$ of variance explained. The three-item scale showed high reliability $(\alpha=.90)$ which is consistent with the study by Bayazit and Mannix (2003). 
Chapter 2

Control variables. We included team size and team composition as control variables in our analyses (van Knippenberg \& Schippers, 2007). Team size was controlled for its potential to impact a team's ability to establish and build upon TMMs, and for its potential to impact on more conflict situations. Team size was measured through the number of team members. For the control variable team composition, we transformed the categorical variable into two dummy variables, using student teams as a baseline since they represent more teams in the simulation.

\section{RESULTS}

\section{Aggregation}

The level of analysis in this study was the team. Thus, all individual survey responses were aggregated to the team level for further analysis. To justify aggregation, we computed $\mathrm{R}_{\mathrm{wg}(\mathrm{j})}$ (James et al., 1993), designed for multiple-item scales, and intraclass correlations coefficients (ICC) (Bliese, 2000). All the values were in accordance with the required criteria: Conflict $\left(\mathrm{R}_{\mathrm{wg}(j)}=0.70 ; \operatorname{ICC}(1)=0.07 ; \operatorname{ICC}(2)=0.27 ; F_{(91,297)}=1.37, p<.05\right)$; satisfaction $\left(\mathrm{R}_{\mathrm{wg}(\mathrm{j})}=0.79 ; \operatorname{ICC}(1)=0.08 ; \operatorname{ICC}(2)=0.29 ; F_{(90,278)}=1.41, p<.05\right)$; and viability $\left(\mathrm{R}_{\mathrm{wg}(\mathrm{)})}=\right.$ $\left.0.80 ; \operatorname{ICC}(1)=0.09 ; \operatorname{ICC}(2)=0.31 ; F_{(90,270)}=1.45, p<.05\right)$. Therefore, individual answers were aggregated to team level.

\section{Hypotheses testing}

Table 2.1. provides the means, standard deviations, and the correlations for all study variables at the team level. As expected, significant positive correlations were found between TMM-similarity in different times. However, the mean values of the similarity of team-TMMs and task-TMMs slightly decreased from time 1 to time 3 (see Table 2.1.). The similarity of team-TMMs at time 1 was correlated significantly with performance, satisfaction, and viability. However, the similarity of task-TMMs at time 1 was not correlated with either dimension of team effectiveness. These results only supported our hypothesis $2 \mathrm{a}, 2 \mathrm{~b}, 2 \mathrm{c}$ regarding the teamTMMs, which partially supported hypotheses 2 . 
Team mental models, relationship conflict and effectiveness over time

Table 2.1. Means, standard deviations, and correlations among all team-level variables

\begin{tabular}{|c|c|c|c|c|c|c|c|c|c|c|c|c|}
\hline & M & $\mathrm{SD}$ & 1 & 2 & 3 & 4 & 5 & 6 & 7 & 8 & 9 & 10 \\
\hline \multicolumn{13}{|l|}{ Control variables } \\
\hline 1. Team size & 4.51 & 0.76 & & & & & & & & & & \\
\hline $\begin{array}{l}\text { 2. Students and } \\
\text { workers }\end{array}$ & 0.14 & 0.35 & .06 & & & & & & & & & \\
\hline 3. Workers & 0.40 & 0.49 & $.32 * *$ & $-.33 * *$ & & & & & & & & \\
\hline
\end{tabular}

Time 1

\begin{tabular}{lllllll}
\hline 4. Task-TMMs & 0.20 & 0.40 & .17 & -.05 & .10 & \\
& & & & & & \\
5. Team-TMMs & 0.35 & 0.41 & .08 & -.18 & $.24 *$ & $.57^{* *}$ \\
\hline
\end{tabular}

Time 2

\begin{tabular}{lllllllll}
\hline 6. Conflict & 1.80 & 0.72 & $-.21 *$ & .12 & -.17 & -.11 & $-.34^{* *}$ \\
\hline
\end{tabular}

\begin{tabular}{|c|c|c|c|c|c|c|c|c|c|c|c|c|}
\hline Time 3 & & & & & & & & & & & & \\
\hline 7. Task-TMMs & 0.19 & 0.34 & .04 & -.08 & -.01 & $.38^{* *}$ & $.41 * *$ & -.18 & & & & \\
\hline 8. Team-TMMs & 0.32 & 0.42 & .08 & -.03 & .06 & $.43^{* *}$ & $.52^{* *}$ & -.19 & $.52 * *$ & & & \\
\hline \multicolumn{13}{|l|}{ Time 4} \\
\hline 9. Performance & 4.52 & 2.28 & .01 & .09 & .08 & .11 & $.22 *$ & $-.27 * *$ & $.24^{*}$ & $.24^{*}$ & & \\
\hline 10. Satisfaction & 5.94 & 0.67 & .01 & .12 & .05 & -.02 & $.26^{*}$ & $-.38^{* *}$ & .17 & .16 & $.34 * *$ & \\
\hline 11. Viability & 5.84 & 0.69 & .06 & .11 & .04 & -.01 & $.24 *$ & $-.41 * *$ & .20 & $.22 *$ & $.23^{*}$ & $.78^{* *}$ \\
\hline
\end{tabular}

Note. $\mathrm{N}=92 ; * p<.05 ; * * p<.01$ 


\section{Chapter 2}

In order to evaluate whether TMMs become more similar over time, we conducted a repeated measures ANOVA. The differences were not significant for either dimension of TMMs from time 1 to time 3 (team-TMMs: $F_{(1,91)}=0.52, p>.05, \eta_{p}{ }^{2}=.01$; task-TMMs: $F_{(1,91)}$ $\left.=0.04, p>.05, \eta_{p}^{2}=.00\right)$. These results did not support our hypothesis 1 .

To evaluate our mediation model, we used the PROCESS macro, developed by Hayes (2013), to test a mediation model with multiple mediators operating in serial. This macro allows us to test the direct effect of TMM-similarity on effectiveness and the indirect effects of TMM-similarity on effectiveness through the mediators, even when there is no association between TMM-similarity and effectiveness. For the path analyses, team size and team composition were entered as control variables. We resampled 5000 times and examined for $95 \%$ confidence intervals (CI). Bootstrapping produces a CI, based on bootstrapped sampling distribution, and we can assume that the indirect effects are significant and that mediation occurred if zero falls outside of the 95 percent CI (Preacher \& Hayes, 2008).

Tables 2.2. and 2.3. provide the path estimates for the models. Concerning hypotheses 3 , the results showed that the similarity of team-TMMs in time 3 did not mediate the relationship of the similarity of team-TMMs in time 1 with effectiveness (performance: 0.50 [CI $=-0.08,1.47]$, Cohen's $f^{2}=0.07$; satisfaction: 0.02 [CI $\left.=-0.15,0.18\right]$, Cohen's $f^{2}=$ 0.07 ; viability: $0.10[\mathrm{CI}=-0.06,0.28]$, Cohen's $f^{2}=0.07$ ). Nonetheless, the similarity of taskTMMs in time 3 mediated the relationship of the similarity of task-TMMs in time 1 with effectiveness (performance: 0.45 [CI $=0.06,1.04]$, Cohen's $f^{2}=0.06$; satisfaction: 0.10 [CI $=$ $0.01,0.25]$, Cohen's $f^{2}=0.04$; viability: 0.12 [CI $=0.02,0.28$ ], Cohen's $f^{2}=0.05$ ). Thus, the results only supported our hypotheses $3 \mathrm{a}, 3 \mathrm{~b}$, and $3 \mathrm{c}$ regarding task-TMMs, which partially supported our hypotheses 3 . 
Team mental models, relationship conflict and effectiveness over time

Table 2.2. Model path estimates for models with team-TMMs

\begin{tabular}{|c|c|c|c|c|c|c|}
\hline & \multicolumn{2}{|c|}{ Performance model } & \multicolumn{2}{|c|}{ Satisfaction model } & \multicolumn{2}{|c|}{ Viability model } \\
\hline & Coefficient & $P$ value & Coefficient & $p$ value & Coefficient & $p$ value \\
\hline Team-TMMs (T1) $\rightarrow$ Effectiveness $^{a}$ & 1.27 & .01 & 0.47 & .01 & 0.44 & .02 \\
\hline Team-TMMs (T1) $\rightarrow$ Effectiveness ${ }^{\mathrm{b}}$ & 0.33 & .67 & 0.26 & .12 & 0.13 & .52 \\
\hline Team-TMMs (T1) $\rightarrow$ Conflict & -0.54 & .00 & -0.56 & .00 & -0.56 & .00 \\
\hline Team-TMMs (T1) $\rightarrow$ Team-TMMs (T3) & 0.56 & .00 & 0.56 & .00 & 0.56 & .00 \\
\hline Conflict $\rightarrow$ Team-TMMs (T3) & -0.01 & .89 & -0.01 & .83 & -0.01 & .83 \\
\hline Conflict $\rightarrow$ Effectiveness & -0.79 & .06 & -0.34 & .01 & -0.38 & .01 \\
\hline Team-TMMs (T3) $\rightarrow$ Effectiveness & 0.90 & .15 & 0.03 & .84 & 0.18 & .22 \\
\hline
\end{tabular}

Note. $n=92$. The coefficients refer to the unstandardized regression coefficient.

a The total effect of team-TMMs in time 1 on team effectiveness without the inclusion of mediator variables.

${ }^{\mathrm{b}}$ The total effect of team-TMMs in time 1 on team effectiveness with the inclusion of mediator variables.

Table 2.3. Model path estimates for models with task-TMM

\begin{tabular}{|c|c|c|c|c|c|c|}
\hline & \multicolumn{2}{|c|}{ Performance model } & \multicolumn{2}{|c|}{ Satisfaction model } & \multicolumn{2}{|c|}{ Viability model } \\
\hline & Coefficient & $p$ value & Coefficient & $p$ value & Coefficient & $p$ value \\
\hline 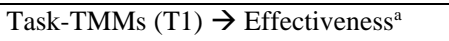 & 0.66 & .23 & -0.03 & .88 & -0.03 & .87 \\
\hline Task-TMMs (T1) $\rightarrow$ Effectiveness ${ }^{\mathrm{b}}$ & 0.11 & .85 & -0.19 & .34 & -0.21 & .21 \\
\hline Task-TMMs (T1) $\rightarrow$ Conflict & -0.12 & .40 & -0.15 & .29 & -0.15 & .29 \\
\hline Task-TMMs (T1) $\rightarrow$ Task-TMMs (T3) & 0.32 & .00 & 0.32 & .00 & .32 & .00 \\
\hline Conflict $\rightarrow$ Task-TMMs (T3) & -0.07 & .15 & -0.08 & .15 & -0.08 & .15 \\
\hline Conflict $\rightarrow$ Effectiveness & -0.81 & .04 & -0.37 & .00 & -0.40 & .01 \\
\hline Task-TMMs (T3) $\rightarrow$ Effectiveness & 1.38 & .01 & 0.31 & .07 & 0.36 & .07 \\
\hline
\end{tabular}

Note. $n=92$. The coefficients refer to the unstandardized regression coefficient.

a The total effect of team-TMMs in time 1 on team effectiveness without the inclusion of mediator variables.

${ }^{\mathrm{b}}$ The total effect of team-TMMs in time 1 on team effectiveness with the inclusion of mediator variables.

Conflict mediated the relationship between the similarity of team-TMMs in time 1 and effectiveness (performance: 0.43 [CI $=0.07,1.02]$, Cohen's $f^{2}=0.10$; satisfaction: 0.19 $[\mathrm{CI}=0.05,0.40]$, Cohen's $f^{2}=0.19$; viability: 0.21 [CI $\left.=0.06,0.45\right]$, Cohen's $f^{2}=0.22$, but did not mediate the relationship between the similarity of task-TMMs in time 1 and effectiveness (performance: 0.10 [CI $=-0.09,0.42]$, Cohen's $f^{2}=0.06$; satisfaction: 0.06 [CI $=$ 
Chapter 2

$-0.04,0.18]$, Cohen's $f^{2}=0.17$; viability: 0.06 [CI $\left.=-0.04,0.20\right]$, Cohen's $\left.f^{2}=0.21\right)$. Thus, once more the results only supported our hypotheses $4 \mathrm{a}, 4 \mathrm{~b}$, and $4 \mathrm{c}$ regarding team-TMMs, which partially supported our hypotheses 4 .

Finally, conflict and TMM-similarity in time 3 did not sequentially mediate the relationship between TMM-similarity in time 1 and team effectiveness at the end of team lifecycle, neither for team-TMMs (performance: 0.00 [CI $=-0.05,0.10]$, Cohen's $f^{2}=0.16$; satisfaction: $0.00[\mathrm{CI}=-0.01,0.01]$, Cohen's $f^{2}=0.26$; viability: 0.00 [CI $\left.=-0.01,0.02\right]$, Cohen's $f^{2}=0.28$ ), nor for task-TMMs (performance: 0.01 [CI $\left.=-0.01,0.09\right]$, Cohen's $f^{2}=$ 0.17; satisfaction: 0.01 [CI $=-0.00,0.02$ ], Cohen's $f^{2}=0.26$; viability: 0.00 [CI $\left.=-0.00,0.02\right]$, Cohen's $\left.f^{2}=0.30\right)$. Thus, our hypotheses $6 \mathrm{a}, 6 \mathrm{~b}$, and $6 \mathrm{c}$ were not supported.

\section{DISCUSSION}

In order to contribute to TMM research, this study evaluated the evolution of TMMsimilarity over a sequence of task episodes, and the role of a dysfunctional team process relationship conflict - on this evolution, and on team effectiveness. Our results provide support for the mediating role of conflict in the relationship between the similarity of teamTMMs at the beginning of team lifecycle and effectiveness. Our results also provide support for the mediating role of task-TMMs in the middle of team lifecycle between task-TMMs at the beginning of team lifecycle and effectiveness. The results did not support our multiple mediation, and we did not find evidence for increasing TMM-similarity over time. We move forward with some explanations.

Our findings suggest that conflict has dysfunctional effects on team effectiveness. Despite the low levels of conflict reported by members, these were enough to diminish the performance, satisfaction and viability, which demonstrated the strength and the dysfunctional impact of conflict (e.g., Bayazit \& Mannix, 2003; Jehn \& Mannix, 2001; Simons \& Peterson, 2000). However, conflict did not have a significant impact on TMM-similarity in the middle of team lifecycle. This may occur because low levels of conflict are not able to diminish, in a short period of time, the shared understanding that members developed about team- and taskaspects of work. Curseu, Kenis, and Raab (2009) showed that when members choose future teammates based on their competences and/or similarities they keep equilibrium in such a way that the disagreements do not deteriorate into relationship conflict. In the Global Management Challenge ${ }^{\circledR}$ members choose their teammates based on their relational preferences or on their 
Team mental models, relationship conflict and effectiveness over time

competences to work. Thus, the low level of relationship conflict may be due to the way teams are formed.

As expected, the similarity of team-TMMs reduces the level of conflict which in turn improves effectiveness. This occurs because members share a common understanding about how work together from the beginning of team lifecycle, which helps them to avoid or manage the disagreements based on personal issues (Thompson, 2004), and to achieve high levels of effectiveness. We also expected that when members have a shared understanding about work objectives and task duties (i.e., when share task-TMMs) they will be more able to avoid personal disagreements (De Dreu \& Weingart, 2003; Thompson, 2004). Nevertheless, the similarity of task-TMMs was not able to reduce relationship conflict. The low average similarity we found in task-TMMs could explain why there was no significant relationship between task-TMMs and conflict. Probably task-TMMs did not have strength to diminish conflict.

Our findings also demonstrated that the similarity of task-TMMs in the middle of team lifecycle mediated the relationship between the similarity of task-TMMs in the beginning of team lifecycle and effectiveness. However, the same results were not obtained concerning the similarity of team-TMMs. Thus, we assume that this mediation only occurred with taskTMMs because teams defined the strategy and the goals accordingly to performance in the beginning of the simulation, which guide all the behaviours and decisions over the simulation. Our mediation models lead us to assume that team- and task-TMMs have different functions on team interaction, but they have similar impact on team effectiveness. Our results are in accordance with Cannon-Bowers and Salas (2001) stated in a theoretical paper: task-TMMs lead to performance, whilst team-TMMs lead to performance through team processes. Owing the results about simple mediations, the results do not allow us to support the double mediation of conflict and TMM-similarity in the middle of interaction.

Only the similarity of team-TMMs had a positive effect on all three criteria of effectiveness. The task-TMMs (in the middle of team lifecycle) only had a positive impact on performance. This means that the shared knowledge about skills, contributions of members, and relational dynamics, is critical to effectiveness from the beginning of team interaction. These results are in contrast to some studies that showed that the similarity of task-TMMs had a stronger effect on performance than team-TMMs (e.g., Lim \& Klein, 2006; Mathieu et al., 2010). This may be due to the way teams are formed. As in the Global Management Challenge ${ }^{\circledR}$ members choose their teammates based on their relational preferences or on their 
Chapter 2

competences to work. Thus, at the start of the team task, members already have a mental model regarding the way they should interact and work together. While task-TMM is specific for each context, team-TMM is not. For this reason, if teams already have a similar teamTMM, this shared understanding is more likely to lead to higher levels of effectiveness. The task-TMM is specific for the context and develops as team members interact within the simulation. Therefore, task-TMM is likely to take more time to develop and reach an optimal level than team-TMM.

TMMs did not become more similar over time. These results are in accordance with other studies that showed the same trajectories (e.g., Levesque et al., 2001; Mathieu et al., 2000). A possible explanation for these results could be related to the feedback given to the team during the simulation. Over the weeks, teams received a management report showing their results only in financial terms, hence they could not derive if they were doing something wrong, or what poor decisions were. In this sense, teams were not able to improve or discuss a common understanding that was wrong but that they believed was correct. In addition, members who participated in our study work together and make decisions over each week of the simulation, while developing other professional or academic activities. Thus, they only have limited time available for interacting with their team members in the simulation. Therefore, it may be difficult improve TMM, and develop professional or academic activities over the simulation. Additionally, in accordance with a recent theoretical adaptation model (Rosen et al., 2011) we think that team learning is essential for TMM update. Through learning behaviours, members ask questions, seek feedback, experiment, and discuss errors and unexpected outcomes (Edmondson, 1999), which may lead to an update of TMM.

\section{Theoretical and research implications}

Our findings are of theoretical interest for several reasons. First, we evaluated our variables over a period of five team performance episodes, and TMMs were evaluated in two different moments. Since there are a limited number of published longitudinal studies on TMMs (e.g., Mohammed et al., 2010), our study adds value to TMM research by showing how team-TMMs diminish relationship conflict and improve effectiveness, and how task-TMMs measured at different points are related to team effectiveness.

In addition, this study is the first to analyse the mediating role of a dysfunctional process, conflict, in the relationship between TMMs and team effectiveness. We find that 
Team mental models, relationship conflict and effectiveness over time

TMM similarity at the beginning of the team lifecycle is related to relationship conflict. Moreover, consistent with previous studies, we find that conflict limits team's cognitive functioning, which in turn lead to a decrease in effectiveness (De Dreu \& Weingart, 2003; de Wit et al., 2012; Jehn \& Mannix, 2001). In other words, conflict limits TMM development and decrease satisfaction, viability, and effectiveness.

Methodologically, in our study we used a path analysis approach that allowed us to test our full conceptual model, instead of testing pieces of our model separately. This methodology allows researchers to understand the relationships between all different aspects of team lifecycle. In addition, this data analysis strategy allows us to test direct and indirect effects between variables, even when there is no association between predictor and outcome.

\section{Practical implications}

Our study also offers practical implications for organizational and business teams. Our findings show the importance of a shared understanding concerning relevant task and team aspects from the beginning of the team lifecycle. Our study offers insights into designing training programs about TMMs through which teams should be instructed in the importance of shared knowledge and strategies needed to enhance its potential. And importantly, these training programs should occur previously to task performance.

Furthermore, our study shows that it is important that teams develop conflict management competencies and know how to use conflict management strategies to prevent conflict (Pazos, 2012) in order to achieve high levels of performance, feel satisfied with team, and be willing to work together in the future. Therefore, training programs about conflict management are also needed. Ideally, team members should be instructed about TMMs and conflict management. This way teams are more likely to develop a shared understanding about how the task should be accomplished and how to interact with each other at the same time they know how to prevent or deal conflicts. In short, the knowledge about how they perform the task and work together will reflect on conflict and impact on team performance (Wood et al., 2011). 


\section{Limitations and future research}

This research was conducted with teams enrolled in a management simulation that, although artificial, nevertheless involved their working together for more than five weeks. They participated in a dynamic simulation, characterized by complex decision-making, where team members needed to focus on several indicators to reach a specific objective. All these factors require high levels of coordination and interaction (Tasa, Taggars, \& Seijts, 2007), similar to real teams in organizations. In addition, some authors have also used these types of teams in their research (e.g., Marks et al., 2002; Mathieu et al., 2000).

Unlike many researchers (e.g., Marks et al., 2002; Mathieu et al., 2000), we did not evaluate the accuracy of TMMs. However, it would be critical to compare the knowledge structures of members with the knowledge of an expert. A future solution could be to ask the members of teams with high classifications in previous editions of the simulation, and compare their answers with those of the participants.

Several authors have discussed how similar TMMs should be in order to increase effectiveness (e.g., Salas et al., 2005; Woehr \& Rentsch, 2003). Possibly, an extreme overlapping of TMMs could lead to a phenomenon similar to groupthink (Janis, 1972), which would be detrimental to effectiveness. In this sense, it will be important to conduct empirical studies to understand how similar TMMs should be, and when the similarity of TMMs is disruptive to teams. In order to do this it will be important to evaluate TMMs at more stages of team interaction and explore the evolution of TMMs over time and evaluate them.

Future studies might also analyse the effect of conflict management strategies. In organizational contexts is crucial that teams learn and use conflict management strategies to prevent relationship conflict while sustaining constructive disagreements (Pazos, 2012). It will be important understand the impact of different conflict management strategies on the relationship between TMMs, relationship conflict, and effectiveness.

Since all measures were obtained from team member respondents, some problems regarding to common-method variance could be point out (Siemsen, Roth, \& Oliveira, 2010). However, some authors defend that method variance is not a problem (Brannick, Chan, Conway, Lance, \& Spector 2010; Lance, Dawson, Bierkelbach, \& Hoffman, 2010), and that the spurious causes of relationships among variables are related to the mixture of methods and constructs and not related to methods themselves (Brannick et al., 2010). Furthermore, 
Team mental models, relationship conflict and effectiveness over time

common method variance is not a problem when not every variables are correlated, what happens in our study (Brannick et al., 2010). Our research also deals with shared biases among variables since we used a longitudinal design (Brannick et al., 2010).

\section{CONCLUSION}

TMMs are an important and emerging field in organizational behaviour. Researchers and professionals who work and/or manage teams must reflect on the relevance and benefits of a shared understanding among the team members. If this sharing aspect of teamwork exists in organizations, workers will achieve high performance levels, will feel satisfied and will want to work together in the future. 



\title{
ChAPTER $3^{4}$
}

\author{
WHY IS YOUR TEAM MORE CREATIVE THAN MINE? \\ THE INFLUENCE OF SHARED MENTAL MODELS ON \\ INTRAGROUP CONFLICT, TEAM CREATIVITY AND \\ EFFECTIVENESS
}

${ }_{4}^{4}$ This chapter is published as:

Santos, C.M., Uitdewilligen, S., \& Passos, A.M. (2015). Why is your team more creative than mine? The influence of shared mental models on intragroup conflict, team creativity and effectiveness. Creativity and Innovation Management, 24, 645-658. DOI: 10.1111/caim.12129 http://onlinelibrary.wiley.com/doi/10.1111/caim.12129/abstract 
Chapter 3

\begin{abstract}
In competitive and dynamic contexts team members need to be creative to ensure that teams achieve high levels of performance and feel satisfied with their work. At the same time, team members need to have a shared understanding regarding relevant aspects related to task accomplishment and team interaction. In this study we investigate the mediating mechanisms of intragroup conflict and creativity in the relationship between shared mental models and team effectiveness (team performance and satisfaction). We tested our model in a sample of 161 teams (735 individuals) performing in a management simulation. We collected data at three time points. Our results suggest that high shared mental models are related to low levels of intragroup conflict, foster creativity, and in turn improve team performance and satisfaction. These findings contribute to a scarce thematic - the relationship between shared mental models and creativity - emphasizing the importance of a shared understanding for creativity and team effectiveness.
\end{abstract}


Why is your team more creative than mine?

\section{INTRODUCTION}

The organizational contexts in which teams operate are becoming increasingly competitive. As a result, teams often need to be creative to achieve their goals, present new solutions or suggest new products (Burke et al., 2006a). Researchers have analyzed team factors that foster team creativity, such as task interdependence, team size, and cohesion (Hülsheger, Anderson \& Salgado, 2009). Yet, an in depth investigation of the impact of team cognitive factors, such as shared mental models, is lacking. Shared mental models (SMM) refer to a common understanding among the team members about relevant task and team aspects of their work (Klimoski \& Mohammed, 1994). Although empirical studies show that SMM foster a variety of team processes and outcomes, such as coordination, adaptation, and performance (e.g., Mathieu et al., 2000; Santos \& Passos, 2013; Uitdewilligen et al., 2013), empirical studies that analyze the effect of SMM on creativity are still missing. As SMM imply a common understanding shared by team members, it is important to analyze whether SMM inhibit creative ideas because team members share the same ideas and do not discuss different points of view, or whether SMM potentiate creative ideas because they facilitate effective coordination and cooperation among the team members.

Literature about the impact of SMM on creativity is scarce. On the one hand, it has been argued that SMM may stifle creativity (Skilton \& Dooley, 2010). When team members have too much overlap in their understanding about task and team aspects of work, this may reduce their ability to innovate and to be creative. On the other hand, previous studies suggest a positive effect of SMM on adaptation, which is closely related to creativity and innovation because teams need to solve problems and create new products in order to be able to adapt (Burke et al., 2006).

Intragroup conflict is likely to play a role in the relationship between SMM and creativity. Conflict results from the tension among team members and involves discrepancies and incompatible goals (De Dreu \& Weingart, 2003; Jehn \& Mannix, 2001). Intragroup conflict may hamper creativity, impede team members' ability to develop new ideas, and thereby decrease team effectiveness (De Dreu \& Weingart, 2003; de Wit et al., 2012; Jehn, 1995; Simons \& Peterson, 2000). A recent study showed that SMM similarity diminishes the level of relationship conflict and in turn improves team effectiveness (Santos \& Passos, 2013). However, empirical studies that analyze the relationship between SMM, conflict, creativity and effectiveness are needed. 
In the present study we investigate the mediating mechanisms of intragroup conflict and team creativity in the relationship between SMM and team effectiveness (team performance and satisfaction). We analyze four types of intragroup conflict: task, relationship, process, and temporal conflict. Analyzing the mediating role of intragroup conflict and creativity allows us to integrate and test alternative theories on how SMM impact team effectiveness (Mathieu, DeShon \& Bergh, 2008a).

\section{THEORETICAL BACKGROUND AND HYPOTHESES}

\section{Shared mental models}

Previous research suggests that SMM impact team performance and satisfaction (e.g., Mathieu et al., 2000, 2010; Santos \& Passos, 2013). Performance is an objective criterion that indicates team level actual task accomplishment. Team satisfaction is an affective concept that indicates the degree to which team members are satisfied with the team experience (Hackman, 1987; McGrath, 1964). SMM foster team effectiveness because they enable team members to anticipate the needs and actions of other team members and to adapt their actions to align with their colleagues as well as with the demands of the task (Cannon-Bowers et al., 1993; DeChurch \& Mesmer-Magnus, 2010). When team members have SMM, they make efficient use of their information and knowledge, produce efficient collective responses to immediate task requirements, and subsequently are able to achieve high levels of performance and feel satisfied with the team experience (Cannon-Bowers et al., 1993; DeChurch \& MesmerMagnus, 2010; Santos \& Passos, 2013).

\section{Shared mental models and intragroup conflict}

Intragroup conflict is a process that occurs when team members perceive their interests and values to be incongruent with those of other members of the team (DeChurch et al., 2013; Jehn \& Mannix, 2001). Three different types of intragroup conflict have been identified in the literature: task, relationship, and process conflict (Jehn, 1995; 1997). Task conflict refers to disagreements among team members related to the content of the tasks, such as differences regarding ideas or opinions (Jehn, 1995). Relationship conflict refers to arguments about personal and social issues that are not directly related to the task and that 
involve negative emotions and tension (De Dreu \& Van Vianen, 2001; Jehn, 1995). Process conflict refers to differences on the procedures by which the task should be accomplished, the distribution of responsibilities, and the structure of delegation within the team (Jehn, 1997). Recently, scholars added a fourth type of conflict: temporal conflict, which refers to intragroup disputes about time, the duration of a task, and the length of time the team should spend on a specific task or goal (Mohammed \& Nadkarni, 2011; Standifer et al., 2015).

Different perspectives exist regarding the impact of intragroup conflict on team functioning. Previous empirical studies consistently show that relationship conflict and process conflict limit team members' ability to share, exchange, and process relevant information, and that they distract team members from engaging in effective task execution (De Dreu \& Weingart, 2003; de Wit et al., 2012; Jehn, 1997; Passos \& Caetano, 2005; Santos $\&$ Passos, 2013). Research on temporal conflict suggests that it increases ambiguity about task deadlines and the sequence of task accomplishment, which disrupts coordination processes and increases team members' frustration (Mohammed \& Nadkarni, 2011; Standifer et al., 2015). Findings about task conflict, however, are inconsistent. Although initial research suggest that task conflict may facilitate innovativeness and high-quality team decisions (Amason, 1996; Jehn, 1995), a meta-analysis by De Dreu and Weingart (2003) shows that task conflict has disruptive effects on team effectiveness. Nevertheless, a recent meta-analysis did not show a strong and negative association between task conflict and team performance (de Wit et al., 2012).

SMM play an important role in the development of intragroup conflict by stimulating constructive conflict and avoiding disruptive conflicts. As teams that have SMM have a common understanding of the task goals, procedures, and strategies, this facilitates coordination among the team members and fosters knowledge about what other members need to accomplish their task. Therefore, members are able to openly discuss ideas and different viewpoints arising over the team lifecycle (Cannon-Bowers et al., 1993; DeChurch \& Mesmer-Magnus, 2010; Santos, Uitdewilligen, \& Passos, 2015a). When team members have SMM and discuss ideas that are aligned with the task and team aspects of their work, they are likely to increase their effectiveness in executing their task. In short, when team members have SMM, they can engage in task conflict situations, solve them, make optimal decisions, achieve high levels of performance, and feel satisfied with their work.

Hypothesis 1 - (a) Task conflict mediates the relationship between SMM and team effectiveness (team performance and satisfaction). 
Team members who have SMM are focused on task accomplishment and discuss aspects related to the task and team interaction that really matter for effectiveness. Consequently, teams avoid disagreements based on personal issues, on team members' responsibilities and on the length of time the team should spend on a specific tasks or goals and thereby achieve high levels of performance and member satisfaction (Santos \& Passos, 2013; Simons \& Peterson, 2000; Standifer et al., 2015). Thus, teams that have SMM experience low levels of relationship, process, and temporal conflict and achieve high levels of performance and their members feel satisfied with their team.

Hypothesis 1 - (b) Relationship conflict, (c) process conflict, and (d) temporal conflict mediate the relationship between SMM and team effectiveness (team performance and satisfaction).

\section{Shared mental models and team creativity}

Creativity refers to the process of "coming up with fresh ideas for changing products, services, and processes so as to better achieve the organization's goals" (Amabile, Barsade, Mueller \& Staw, 2005, p. 367). Creativity requires originality and effectiveness, as "original things must be effective to be creative" (Runco \& Jaeger, 2012, p. 92). Past research has demonstrated that team input variables, such as task interdependence, and job-relevant diversity stimulate team creative processes (Hülsheger et al., 2009; Van der Vegt \& Janssen, 2003; West, 2002). Team processes, such as communication patterns, task orientation and intragroup conflict, also have an impact on creativity and innovation (De Dreu, 2006; Jehn, 1995; West \& Anderson, 1996).

Despite these important findings in creativity research, a relevant discussion exists on the effect of SMM on creativity that needs clarification. Skilton and Dooley (2010) pose that SMM may stifle creativity. They argue that particularly when team members work together on creative projects, they internalize and synchronize their SMM, which over time become resistant to change. In subsequent projects team members may avoid discussing novel ideas and diverging points of view in order to avoid conflict and not to disrupt the status quo (Skilton \& Dooley, 2010). In short, Skilton and Dooley (2010) argue that the common understanding shared by team members inhibits innovative and creative ideas.

Contrastingly, a number of empirical studies have shown that SMM positively foster team adaptation, which includes innovation as a subfacet (Burke et al., 2006a; Resick et al., 
Why is your team more creative than mine?

2010b; Uitdewilligen et al., 2013). When team members have SMM, they are able to adapt their routines when they are confronted with complex and dynamic task environments (Kozlowski et al., 2001; Resick et al., 2010b). Hülsheger and colleagues (2009) argue that when teams have SMM, the positive relationship between job-relevant diversity and innovation might be strong, because then team members' working styles are aligned, they agree on team norms, and they are coordinated and willing to cooperate with each other (Bledow, Frese, Anderson, Erez \& Farr, 2009; Kozlowski \& Bell, 2003). Furthermore, team members who have SMM are able to generate, create and implement new ideas or products that are aligned and in accordance with the requirements of the task and the needs of the team (Burke et al., 2006a; Hülsheger et al., 2009). Consequently, teams achieve high levels of performance and team members feel satisfied.

Although team factors directly impact team creativity, they also impact the extent to which individuals can be creative, which in turn facilitates team effectiveness. Research suggest that when team members "are open to new ideas, constructively challenge one another, effectively communicate and provide feedback, successfully manage conflict, trust and help each other, and share a commitment to their work" (DiLiello et al., 2011, p. 155) team members perceive support for creativity, which fosters team and organizational innovation and effectiveness (Amabile, 1988; DiLiello, et al., 2011). In this sense, we argue that a team work environment where members have a similar understanding regarding relevant team and task aspects of their work fosters team members' creativity. SMM and creativity, in turn, have a positive impact on the ability of the team to perform and the extent to which its members feel satisfied with the team.

Hypothesis 2 - Creativity positively mediates the relationship between SMM and team effectiveness (team performance and satisfaction).

\section{Shared mental models, intragroup conflict and team creativity}

A number of empirical studies have given special attention to the relationship between task conflict on creativity (Badke-Schaub, Goldschmidt \& Meijer, 2010; Chen, 2006; De Dreu, 2006; Pelled, Eisenhardt \& Xin, 1999). Findings suggest that task conflict fosters creativity because when team members disagree about central components of the task, they are likely to explore opposing ideas, and discuss diverging viewpoints, which enhances the 
Chapter 3

generation of new ideas (Badke-Schaub et al., 2010; Chen, 2006). On the contrary, relationship conflict hampers creativity because it limits team members' ability to communicate, discuss and share information (Chen, 2006; Jehn, 1995; Simons \& Petters, 2000).

We argue that teams that have SMM, and experience high levels of task conflict and low levels of relationship, process and temporal conflict, are likely to create new solutions for problems or unexpected situations and to achieve high levels of team effectiveness. As team members share a common understanding of relevant aspects of their work, they discuss issues related to task accomplishment and team goals, and they avoid disruptive conflicts (Jehn, 1995; Santos \& Passos, 2013; Simons \& Peterson, 2000). Consequently, as team members think about new ideas and solutions, they achieve high levels of performance and feel satisfied with the team. Thus, we expect a relationship between SMM, team conflict, creativity and effectiveness.

Hypothesis 3 - (a) Task conflict, (b) relationship conflict, (c) process conflict, (d) temporal conflict and creativity sequentially mediate the relationship between SMM and team effectiveness.

The research model is represented in Figure 3.1.

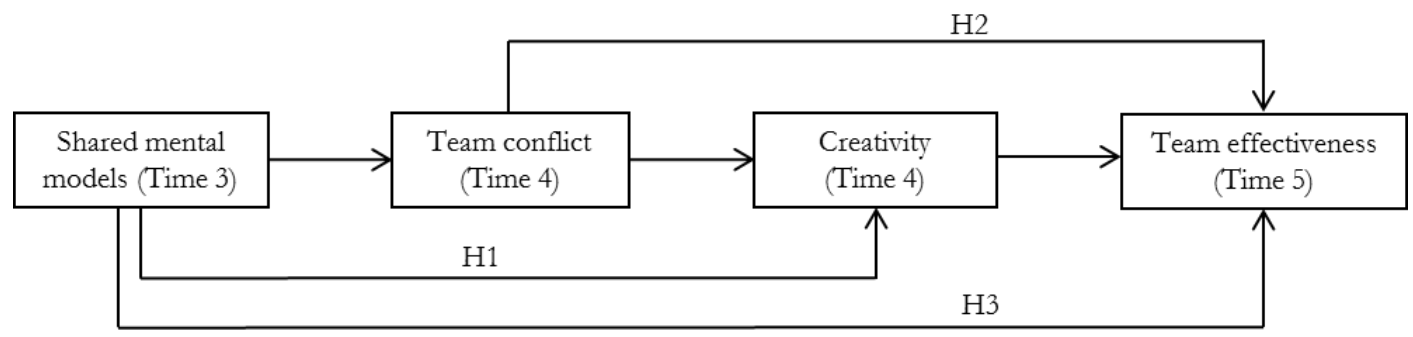

Figure 3.1. Hypothesized model. 
Why is your team more creative than mine?

\section{METHOD}

\section{Participants}

A total of 161 teams (735 individuals) participated in this study. All teams were enrolled in a national management and strategy simulation for a 5-week period. The teams were composed of workers $(42.6 \%)$, university students $(41.1 \%)$, or a mix of workers and students $(16.4 \%)$. Team sizes ranged from three to five members, with an average of 4.67 (s.d. $=0.62)$. The average age was 29 years (s.d. $=8.42)$ and $67.4 \%$ of the participants was male.

\section{Simulation}

We collected data using the business simulation the Global Management Challenge ${ }^{\circledR}$. In this simulation teams run fictitious companies that have the objective of gaining the highest share price on the simulated stock exchange. The simulation has been running for over thirty years, and many top companies encourage their employees to take part in it. The simulation comprises four stages: first round, second round, national final, and international final. We collected data in the first round of the simulation that takes place across five consecutive weeks and involves a larger number of teams. Before the beginning of the simulation teams receive a manual that explains how the simulation works, and a management report about the companies they will run. This information can be used over the simulation. Further, approximately one month before the beginning of the simulation participating teams enroll in two training sessions. The teams were assigned to a group consisting of a maximum of eight teams representing a competitive market, in which they had to compete with one another. The simulation lasts five weeks, with each week simulating three months. In each quarter teams make decisions on the marketing, production, personnel, purchasing, and finance of their fictional company. The simulation algorithm computes the effect of these decisions on the companies' financial indicators, their share price and on their ranking relative to the other teams. This information is presented to the team in the form of a management report to the teams after each quarter.

Team members are free to assemble their own team. Because members might know each other from their university (for student teams), or from their jobs (worker teams) some teams may have worked together before. The mix teams were formed by students and 
Chapter 3

workers. For these teams it is less likely that members have worked together previously. Companies may ask students to join their teams as part of a recruitment process as this enables them to analyze the behaviors, competencies and skills of these students.

\section{Procedure}

Team members answered two different on-line questionnaires during the simulation. Performance was provided by the company responsible for the simulation. The company authorized data collection and informed participants about the research, whereas the authors of the study were responsible for sending the link to the questionnaires and for collecting the data. The link to the questionnaires was sent to the team members by e-mail at different time moments in the simulation period. Participants individually answered the questionnaires before receiving the management report with the feedback about their decisions.

\section{Measures}

Shared mental models. Based on the four types of models identified by Cannon Bowers and colleagues (1993), we developed a four-item scale to evaluate SMM. On a 7-point Likert scale ( $1=$ totally disagree, $7=$ totally agree) the participants indicated how much they agreed with each of the statements. A list of the statements is provided in the Appendix B. We performed an exploratory factor analysis which revealed only one factor with 81.24 percent of variance explained. A confirmatory factor analysis (CFA) executed in Mplus (Muthén \& Muthén, 19982010) indicated an acceptable goodness of fit index, with all indices falling within acceptable ranges (Hu \& Bentler, 1999; Schreiber, Stage, King, Nora \& Barlow, 2006): $\chi^{2}(2)=55.73, p=$ .00 ; RMSEA $=.00 ;$ CFI $=.97$; TLI $=.90 ;$ SRMR $=.02$. SMM were measured in the third week of the competition $(\alpha=.92)$.

Intragroup conflict. Relationship, task, and process conflicts were measured with twelve items from the Intragroup Conflict Scale by Jehn (1995, 1997). Temporal conflict was measured with a modified three-item scale by Yang (2009) based on the original process conflict scale developed by Jehn (1995) and Shah and Jehn (1993). On a 7-point scale (1 = never, $7=$ always), the participants indicated how often each behavior occurred in their team. A 
list of the statements is provided in the Appendix B. Conflict was measured in the fourth week of the competition $\left(\alpha_{\text {relationship }}=.96 ; \alpha_{\text {task }}=.87 ; \alpha_{\text {process }}=.92 ; \alpha_{\text {temporal }}=.95\right)$.

Creativity. Creativity was measured with the self-perceived creativity and creative selfefficacy (5 and 6 items respectively) scales by DiLiello and colleagues (2011). On a 7-point scale $(1=$ totally disagree; 7 = totally agree), the participants rated the extent to which they agreed with each sentence. A list of the sentences is provided in the Appendix B. We used the two scales together, because an exploratory factor analysis revealed only one factor with 78.14 percent of variance explained. A CFA was implemented by Mplus (Muthén \& Muthén, 19982010) which presented an acceptable goodness of fit index since all indices fell within acceptable ranges ( $\mathrm{Hu} \&$ Bentler, 1999; Schreiber et al., 2006): $\chi^{2}(35)=327.39, p=.00$; RMSEA $=.00 ;$ CFI $=.95 ;$ TLI $=.93$; SRMR $=.03$. Creativity was measured in the fourth week of the competition $(\alpha=.97)$.

Performance. Performance was operationalized as the share price at the end of the simulation. The share price was given in Euros and was automatically calculated by the simulation software. The simulation also automatically provided the team's relative position in the group, ranging from first to eighth. We operationalized performance by recoding the share price on a 1 to 8 point-scale through the percentile values - we asked for the cut off points for eight equal groups. We created a new variable based on the values of the eight groups, which was used in the mediation analyses. The lowest share prices correspond to lower values and the highest share prices correspond to higher values. This performance measure was discussed and constructed on the basis of recommendations of the developers of the management simulation.

Team satisfaction. Team satisfaction was measured with eight items adapted from the Job Satisfaction Scale by Spector (1997). On a 7 -point scale $(1=$ very dissatisfied; $7=$ very satisfied), the participants rated how much they were satisfied with different aspects of their teamwork. A list of the statements is provided in the Appendix B. Satisfaction was measured in the fifth week of the competition $(\alpha=.98)$.

Control variables. We included team size, team composition (workers, university students, or both), and familiarity as control variables in our analyses (Humphrey, Morgeson \& Mannor, 
Chapter 3

2009; van Knippenberg \& Schippers, 2007). We controlled for team size because it can impact a team's ability to establish and build upon mental models. Team size was measured as the number members in the team. For the control variable team composition, we transformed the categorical variable into two dummy variables, using the workers as a baseline, since they represented more teams in the competition. Familiarity was measured as the percentage of team members that already knew each other before the start of the simulation.

\section{RESULTS}

\section{Aggregation}

The level of analysis in this study was the team. Thus, all individual survey responses were aggregated to the team level for further analysis. To justify aggregation, we computed $\mathrm{R}_{\mathrm{wg}(\mathrm{)})}$ (James et al., 1993), designed for multiple-item scale, and intraclass correlation coefficients (ICC) (Bliese, 2000). All the values were in accordance with the required criteria (see Table 3.1.). Therefore, individual answers were aggregated to team level. 
Why is your team more creative than mine?

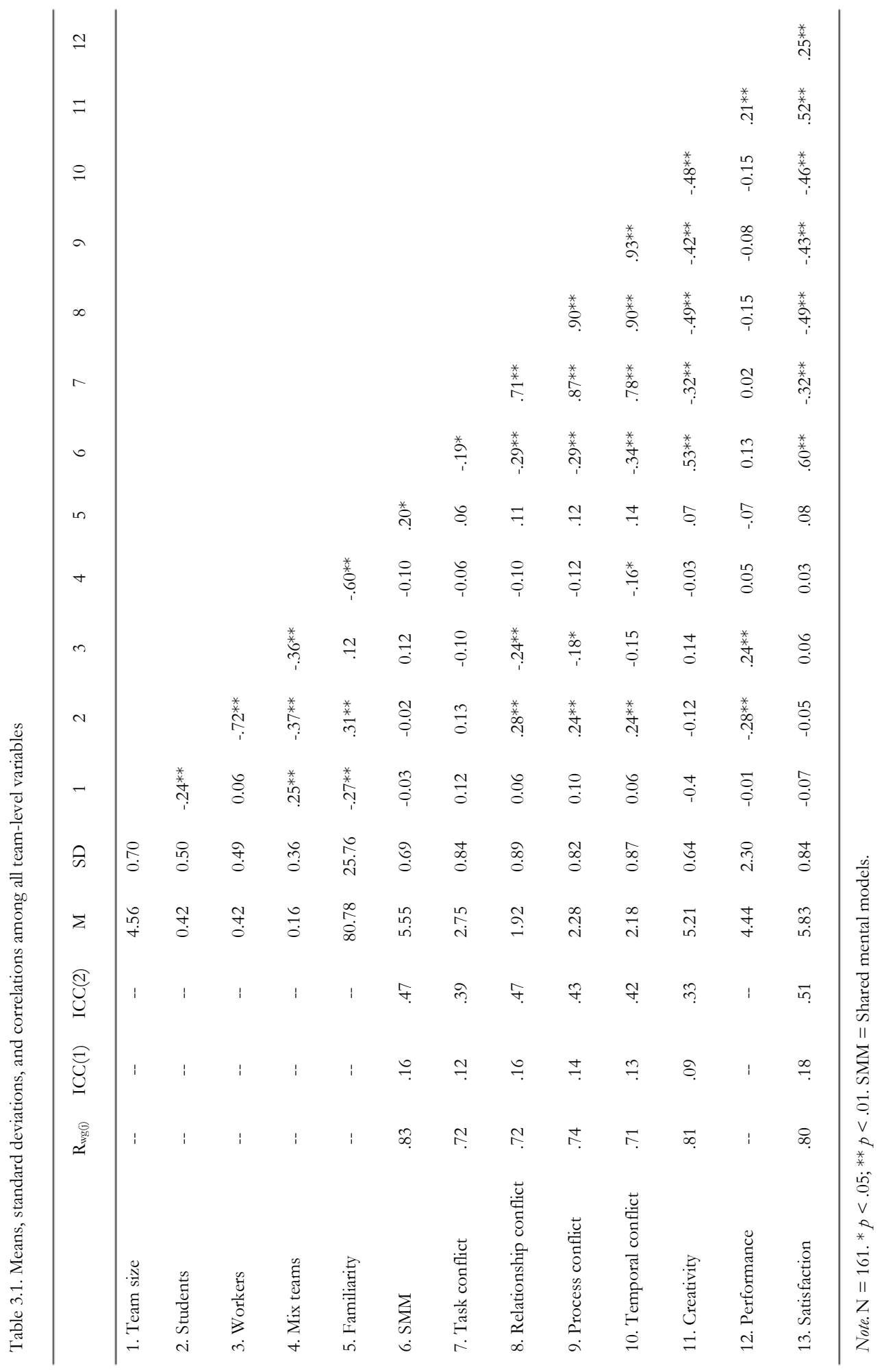


Chapter 3

\section{Hypotheses testing}

Table 3.1. provides the means, standard deviations, and the correlations for all study variables at the team level. The results show a negative and significant correlation between mental models and the four types of intragroup conflict. The results show a positive and significant correlation between SMM and creativity $(r=0.53, p<.01)$, as well as satisfaction $(r$ $=0.60, p<.01)$. The four types of conflict were negative and significantly correlated with creativity and satisfaction. Creativity was positively and significantly correlated with team effectiveness $\left(r_{\text {performance }}=0.21, p<.01 ; r_{\text {satifaction }}=0.52, p<.01\right)$. Regarding the control variables, familiarity was positively and significantly correlated with mental models $(r=.20, p<.05)$.

To evaluate our research model with multiple sequential mediators, we used the PROCESS macro, developed by Hayes (2013). This macro allows for testing the indirect effects of SMM on effectiveness through conflict and creativity, even when there is no association between SMM and effectiveness. Team size, team composition, and familiarity were entered as control variables. We resampled 5000 times and examined for $95 \%$ confidence intervals (CI). We can assume that the indirect effects are significant and that mediation occurred if zero falls outside of the 95 percent confidence interval (Preacher \& Hayes, 2008).

Table 3.2. provides the path estimates for the models. Hypotheses 1 (a-d) proposes that intragroup conflict mediates the relationship between SMM and team effectiveness. Intragroup conflict mediated the relationship between SMM and satisfaction (task: 0.05 [CI = 0.01, 0.13]; relationship: 0.11 [CI $=0.05,0.22]$; process: 0.09 [CI $=0.03,0.19]$; temporal: 0.10 $[C I=0.04,0.20])$. Intragroup conflict did not mediate the relationship between SMM and performance (task: -0.13 [CI $=-0.40,0.02]$; relationship: $-0.00[\mathrm{CI}=-0.25,0.24]$; process: $0.08[\mathrm{CI}=-0.38,0.14]$; temporal: $0.02[\mathrm{CI}=-0.27,0.30])$. Hypotheses $1 \mathrm{a}, 1 \mathrm{~b}, 1 \mathrm{c}$ and $1 \mathrm{~d}$ were partially supported. 
Why is your team more creative than mine?

Table 3.2. Model path estimates for models with task, relationship, process and temporal conflict

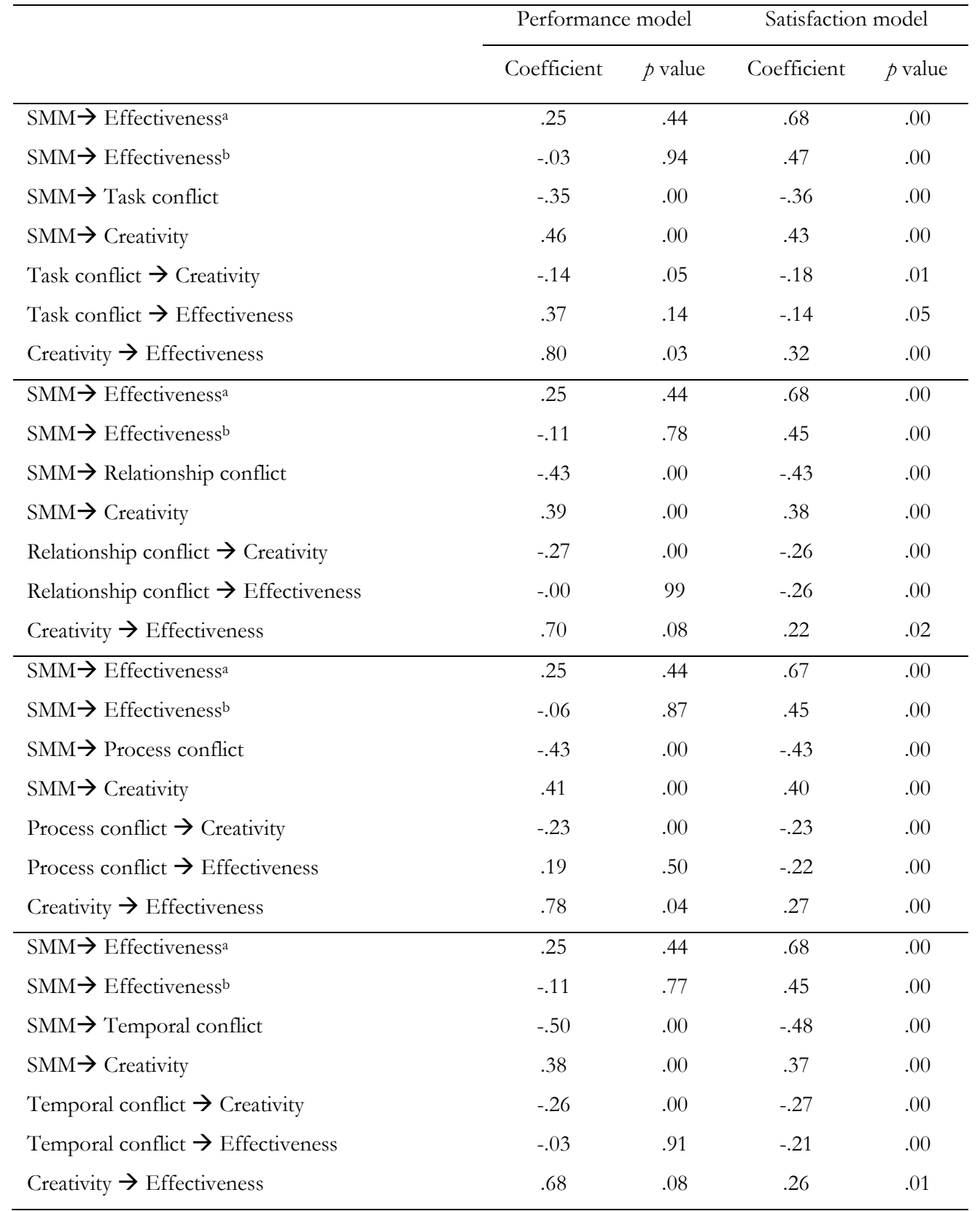

Note. $n=161$ teams. SMM $=$ Shared mental models. The coefficients refer to the unstandardized regression coefficient.

a The total effect of SMM on team effectiveness without the inclusion of mediator variables.

b The total effect of SMM on team effectiveness with the inclusion of mediator variables. 
Chapter 3

Hypothesis 2 proposes that creativity positively mediates the relationship between SMM and team effectiveness. The results showed that creativity mediated the relationship of SMM with performance $(0.37[\mathrm{CI}=0.09,0.73])$, and satisfaction $(0.14[\mathrm{CI}=0.05,0.27])$. Hypothesis 2 was supported.

Hypotheses 3 (a-d) pose that intragroup conflict and creativity sequentially mediate the relationship between SMM and team effectiveness. The results showed that task conflict and creativity mediated the relationship of SMM with performance $(0.04[\mathrm{CI}=0.01,0.16])$ and satisfaction $(0.02[\mathrm{CI}=0.01,0.05])$. Hypothesis $3 \mathrm{a}$ was partially supported. Relationship conflict and creativity mediated the relationship of SMM with performance $(0.08$ [CI $=0.01$, $0.24]$ ) and satisfaction $(0.03[\mathrm{CI}=0.01,0.07])$. Hypothesis $3 \mathrm{~b}$ was supported. Process conflict and creativity mediated the relationship of SMM with performance $(0.08$ [CI $=0.01,0.20])$ and satisfaction $(0.03$ [CI $=0.01,0.06])$. These results support hypothesis $3 \mathrm{c}$. Temporal conflict and creativity mediated the relationship of SMM with performance $(0.09$ [CI $=0.01,0.27])$ and satisfaction $(0.03[\mathrm{CI}=0.01,0.08])$. Hypothesis $3 \mathrm{~d}$ was supported.

\section{DISCUSSION}

This study presents important findings for teams functioning in highly competitive and dynamic environments where team members need to be creative and present innovative solutions. Our study shows the importance of SMM in constraining the level of intragroup conflict, in stimulating creativity, and in fostering team effectiveness.

Our findings suggest that SMM diminish the level of conflict and in turn improve team satisfaction. However, in contrast with our hypothesis, SMM did not improve team performance by decreasing intragroup conflict. These results are contrary to our expectations, which postulated that when team members have SMM they engage in task conflict because they are able to discuss ideas and divergent viewpoints that arise over the team lifecycle as they clearly know the task goals and procedures as well as the needs of other members. Our findings may suggest that as team members share an understanding about the task procedures they previously define all the aspects related to task execution, and discuss different ideas and points of view. Thus, in the middle of team lifecycle all these aspects are clearly defined and they do not need to discuss these again.

SMM diminish the level of intragroup conflict and in turn improve team satisfaction. Our research reveals important findings on a mediator that is scarcely analyzed in SMM 
research. It appears that SMM impede discussions about personal issues, deadlines, and task delegation because team members are focused on task accomplishment. Consequently, team members are more likely to feel satisfied with the teamwork experience (Rentsch \& Klimoski, 2001). However, SMM did not improve team performance via team conflict. These results are contrary to our expectations, and may suggest that intragroup conflict is more relevant as a mediating mechanism in the relationship between SMM and team satisfaction, than in the relationship between SMM and performance. The social issues, deadlines, task procedures, and task delegations that teams discuss in the middle of team lifecycle are important for the level of satisfaction team members feel at the end of the task accomplishment, but they do not impact the level of performance.

Finally, our findings show that intragroup conflict and creativity act as mediating mechanisms between SMM and team effectiveness. These results are in accordance with our expectations and show that as team members have SMM they do not engage in conflict behaviors, which allows them to be creative and in turn to achieve high levels of performance and feel satisfied with the team.

\section{Theoretical and research implications}

Our research represents a contribution to the study of SMM by showing that this cognitive construct positively influences team creativity, and in turn team effectiveness. Thus far, different perspectives exist regarding the impact of SMM on creativity. While some authors argue that SMM block creativity (Skilton \& Dooley, 2010), other authors argue that when team members have SMM they are more likely to be creative because they are coordinated, willing to cooperate with each other, and they trust and help each other (DiLiello et al., 2011; Hülsheger et al., 2009). Our findings support the latter perspective and show that in organizational contexts where team members have SMM, team members develop creative ideas, because their working styles are aligned, and they agree on important aspects of taskwork and teamwork (Hülsheger et al., 2009). Therefore, team members are able to achieve high levels of performance and feel satisfied with their work. However, more research about these topics is needed.

Our study analyzes temporal conflict, which represents a recent dimension of intragroup conflict with important implications for team functioning. Increasingly teams are pressured by time, work on tight deadlines, and need to distribute their time and resources 
Chapter 3

over different projects. Thus, it is important that teams avoid temporal disagreements and know how to work from the beginning of team lifecycle. More research about temporal conflict is needed.

\section{Practical implications}

Our study offers a number of insights that companies may use in order to help their teams to improve their creativity and effectiveness. First, our study offers insights into the importance of fostering SMM and creativity in organizational teams. Our findings show that when team members have SMM, team members develop creative ideas that are in accordance with the requirements of the task and the needs of the team. Furthermore, our findings show that teams need to avoid conflict situations to be creative and to perform optimally. This implies that teams would benefit from receiving information about the importance of developing SMM and avoiding and managing conflicts.

Previous research suggests that the development of SMM may be promoted by having team members engage in collective planning prior to task performance (Stout et al., 1999). In addition, a number of studies emphasize the important role the team leader plays in fostering the development of SMM. Marks and colleagues (2000) found that team leaders can help teams to develop SMM by providing briefings before the actual performance episode. Lorinkova, Pearsall, and Sims (2013), found that teams with empowering leaders develop more similar mental models than teams with directive leaders. So, when creativity is important for goal attainment, team leaders may be instructed to develop an empowering leadership style, in which they involve all team members in the goal setting and decision making process.

Some interventions can be used particularly for (interdisciplinary) teams that work virtually, for instance, international teams. A mind mapping intervention can be used where team members should, individually, think about or write the main actions and/or the main information they need to perform well in the task (Rentsch, Delise, Salas \& Letsky, 2010). Then, each team member should explain to each other the meaning of each action. In the next step, team members should collectively make the connection among those concepts, thereby creating a shared mental representation. Face-to-face teams may do this intervention together and make a "physical" mind map, for instance on a white board. International or virtual teams may develop this shared knowledge object for instance in a video-conference using virtual collaboration software. This intervention is likely to facilitate team members' understanding of 
each other's work, align their strategy, and increase the knowledge of each other skills, leading to a shared knowledge about the main aspects of the task and teamwork.

To prevent conflict situations, team leaders and team members need to learn strategies that could help them to discuss important aspects of work, deadlines, and task delegation in a way, and in a time moment, that would not interfere with team functioning. Team members and leaders need to develop conflict management competencies and learn how and when use those strategies to prevent dysfunctional conflict situations. Behfar, Peterson, Mannix and Trochin (2008) identified a number of strategies that can help teams to reduce conflict and improve performance and satisfaction. For instance, to prevent task conflict, team members can discuss or debate different ideas and opinions in order to achieve consensus, ensuring that all members have a chance to explain their view points. To prevent relationship conflict, team members can be trained to avoid taking disagreements in a personal way. Conflict management training can help team members focus on the content of the arguments instead of on the possibly unpleasant style in which these are formulated. To prevent process conflict, team members can schedule meetings in which they discuss and ensure the quality of the work, and prevent or plan for potential future problems. They can also assign a member (for instance the team leader) to allocate responsibilities, or they can provide team members with autonomy choose which specific task they want to execute (Behfar et al., 2008). Preventing temporal conflict is not only important for teams that work together in the same place and time, but is particularly important for international or virtual teams (Montoya-Weiss, Massey \& Song, 2001). Both types of teams can use temporal coordination mechanisms that help them to communicate and coordinate effectively. For instance, they can create rules and procedures to prevent time management issues, they can establish consensus on the allocation of time to the different team tasks, and they can schedule deadlines to align the pace and effort among the team members (Behfar et al., 2008; MontoyaWeiss et al., 2001).

\section{Limitations and directions for future research}

The management simulation is an artificial scenario that has some limitations for the external validity of the findings. For instance, team members may not be fully engaged in the simulation, or may not work for the collective goal. However, similar to real teams in organizations, teams need to make several complex decisions where team members need to 
Chapter 3

focus on different indicators to achieve a specific objective, and need to work together in an episodic way over five weeks (Mathieu et al., 2000; Mathieu \& Rapp, 2009). This scenario, as in the real world, requires high levels of interaction and coordination. In the real world we could expect that teams would share a stronger SMM than in the simulation because team members know each other for a long period of time, and they are familiar with the tasks they need to perform, as well as with the preferences and abilities of each other. Thus, the effect of SMM fostering creativity, reducing intragroup conflict, and in turn improving team effectiveness could be stronger than in the simulation.

We analyzed creativity through individual level measures: self-perceived creativity and creative self-efficacy, which were aggregated to the team level. As our study regards to a team level study, we should analyze creativity through a team level measure (Costa et al., 2013). However, individuals still need to be creative for a team to perform well. As we measured and considered team members perception of their own creativity, our findings can help team members and leaders to identify creativity gaps and help human resources managers to design training programs and interventions in order to develop some untrained creative skills and competencies (DiLiello et al., 2011). Despite this advantage, future studies should analyze creativity through team level measures.

The creativity measure we used in this study reflects self-perceived creativity and creative self-efficacy, which may refer to a fixed characteristic of the person. Despite research has shown that team factors influence individuals perception of their own creativity (Amabile, 1988; DiLiello et al., 2011), fixed characteristics of the person normally are not easily influenced by the environment. We could measure creativity before the teamwork experience has started to test its relationship with mental models, conflict and team effectiveness.

Although our study shows that SMM do not impede creative ideas, it is important understand when too much overlapping of mental models become disruptive to creativity and to team functioning. If all team members have an exact replication of each other's mental models the creation of new solutions and ideas can be threatened (Salas et al., 2005). Future studies should analyze these questions. 
Why is your team more creative than mine?

\section{CONCLUSION}

In increasingly competitive contexts, teams need to share an understanding about the way they work and interact. Simultaneously they need to present new ideas and solutions in order to achieve high levels of performance and compete with other teams and organizations. Our study provides important implications that should be taking into account by team leaders and organizational managers who want that their teams present creative ideas, experience low levels of conflict and achieve high levels of effectiveness. 

CHAPTER $4^{5}$

SHARED TEMPORAL COGNITIONS AS SUBSTITUTE FOR TEMPORAL LEADERSHIP: AN ANALYSIS OF THEIR EFFECTS ON TEMPORAL CONFLICT AND TEAM PERFORMANCE

${ }_{5}^{5}$ This chapter is published as:

Santos, C.M., Passos, A.M., Uitdewilligen, S., \& Nübold, A. (2016). Shared temporal cognitions as substitute for temporal leadership: An analysis of their effects on temporal conflict and team performance. The Leadership Quarterly. Advance online publication. DOI: 10.1016/j.leaqua.2015.12.002.

http://www.sciencedirect.com/science/article/pii/S1048984315001502 


\title{
Chapter 4
}

\begin{abstract}
Due to changing organizational demands, team leaders increasingly need to engage in temporal leadership behaviors in order to coordinate team members' efforts, avoid time related conflicts and ensure that teams perform well. Simultaneously, temporal conflicts and team performance are impacted by team members' shared temporal cognitions. In this study, we investigate the effect of temporal leadership and shared temporal cognitions on team performance via temporal conflict and test whether the impact of temporal leadership on temporal conflict may be substituted by shared temporal cognitions. Our study was conducted in a management simulation involving 142 teams working on a task over 5 weeks. Results suggest that temporal conflict mediates the relationship between temporal leadership and team performance as well as between shared temporal cognitions and team performance. Further, we found support for the idea that shared temporal cognitions function as a substitute of temporal leadership for reducing temporal conflict in teams.
\end{abstract}




\section{INTRODUCTION}

Over the last decades, organizations have faced a number of changes that pose novel challenges for teams in temporally integrating and coordinating their work processes. As the organizational contexts in which teams operate have become increasingly competitive, teams often have to work under tight deadlines while managing multiple projects at the same time (Gevers et al., 2006; Waller et al., 2001). As a result of these challenges, disagreement among team members on temporal issues or ambiguity over the temporal coordination of actions may arise and create tension and dissatisfaction among members, accumulating in temporal conflict (Jansen \& Kristof-Brown, 2005; Mohammed \& Nadkarni, 2011; Standifer et al., 2015). Temporal conflict refers to intragroup disputes about time, the duration of a task, and the length of time the team should spend on a specific task or goal (Gevers \& Petters, 2009; Mohammed \& Nadkarni, 2011). Previous research has demonstrated that temporal conflict is detrimental to a team's ability to attain high quality outputs in time (Mohammed \& Nadkarni, 2011; Standifer et al., 2015). Thus, in order to ensure that teams perform well, temporal conflict in teams needs to be minimized (Cataldo \& Herbsleb, 2013; Schmidt, Bienvenu, Fitzpatrick, \& Amazeen, 1998; Standifer et al., 2015).

In order to avoid conflict over temporal issues in teams, such as meeting times and deadlines, it has become increasingly important to manage the time available to the team and its members. In the present study we propose two different solutions for avoiding or minimizing temporal conflict in teams, namely, temporal leadership and teams' shared temporal cognitions. Importantly, those two concepts represent different coordination mechanisms, an explicit one (temporal leadership) and an implicit one (shared temporal cognitions), both allowing team members to manage their interdependencies, avoiding conflict, and increasing performance (Rico et al., 2008).

Temporal leadership refers to "leader behaviors that aid in structuring, coordinating, and managing the pacing of task accomplishment in a team" (Mohammed \& Nadkarni, 2011, p. 492). Temporal leadership is related to the functional leadership approach that postulates that leadership should aim to fulfill the team's needs in order to promote team effectiveness (Zaccaro et al., 2001). Mohammed and Nadkarni (2011) operationalized the concept of temporal leadership showing that when team leaders employ strong temporal leadership behaviors they are able to reduce the problems and/or enlarge the benefits of diversity in time urgency (feeling chronically hurried and worried about the passage of time) and pacing style 
(people's preference for the allocation of time to accomplish tasks under deadlines). When team leaders employ strong temporal behaviors, such as allocation of temporal resources, definition of clear schedules and deadlines, and synchronization of activities, they create temporal synergies and reduce the problems related to time urgency diversity. In addition, strong temporal leadership behaviors convert pacing style diversity into a constructive team experience, avoiding team performance breakdowns (Mohammed \& Nadkarni, 2011). In sum, strong temporal leadership "dynamically adjusts individual work cycles and coordinates a team so that work is finished on time" (Mohammed \& Nadkarni, 2011, p. 494).

An alternative, but implicit solution for constraining temporal conflict and ensuring high team performance, is the development of shared temporal cognitions (Gevers et al., 2006; Mohammed \& Nadkarni, 2014; Standifer et al., 2015). Shared temporal cognitions represent a team's shared ideas with regard to "the temporal aspects of a specific group task, such as the importance of meeting the deadline, (sub)task completion times, and the appropriate timing and pacing of task activities" (Gevers et al., 2006, p. 54). When team members have similar perspectives about deadlines and there is little ambiguity about the timing of actions, this is likely to result in high temporal synchronization and low levels of temporal conflict among members. A number of studies have shown the importance of shared cognitions for facilitating the coordination of actions among team members and team performance (e.g., DeChurch \& Mesmer-Magnus, 2010; Mathieu et al., 2000; Santos \& Passos, 2013; Uitdewilligen et al., 2013).

Recently, team researchers have called for studies that consider the interplay between implicit and explicit coordination mechanisms in order to reveal potential interaction effects (like substitution and complementation) on team performance (Espinosa et al., 2004; Rico et al., 2008). Specifically, it has been suggested that implicit coordination mechanisms (like teams' shared cognitions) may substitute explicit mechanisms during team interaction (like temporal leadership), particularly when teams are under time pressure (Espinosa et al., 2004). This idea is also in line with substitutes for leadership theory, suggesting that followers' knowledge and understanding may make leadership redundant (Dionne, Yammarino, Howell, \& Villa, 2005; Kerr \& Jermier, 1978). When team members have strong shared temporal cognitions as an internal resource, they do not need their leader to employ strong temporal leadership as they already have strong shared ideas for pacing, deadlines, and the alignment of task activities, and decreased temporal ambiguity. This renders the need for an external source of structuring temporal aspects (i.e., temporal leadership behaviors) less important for the team members. 
In the present study we examine the mediating mechanism of temporal conflict in the relationship of temporal leadership (Hypothesis 1) and shared temporal cognitions (Hypothesis 2) on team performance. Further, as past research suggests (Nübold, Muck, \& Maier, 2013; Podsakoff, MacKenzie, \& Bommer, 1996), a substitution effect is best tested when researchers "examine whether the substitutes variables moderate relationships between leader behavior and subordinate criterion variables" (Podsakoff et al., 1996, p. 380). Therefore, we analyze the moderating influence of shared temporal cognitions on the relationship between temporal leadership and temporal conflict (Hypothesis 3). Thus, we analyze whether shared temporal cognitions may function as a substitute for temporal leadership in avoiding temporal conflict. Finally, we analyze the interaction effect of temporal leadership and shared temporal cognitions on performance via temporal conflict with a mediated moderation model (Hypothesis 4).

The contributions of this paper are twofold. First, our study contributes to leadership theory in two ways: By focusing on the temporal aspects of leader behavior and incorporating temporality into a leadership construct (Mohammed \& Alipour, 2014), we account for the changing organizational demands and the increasing need to further understand how temporal aspects impact organizational functioning. This may prove especially important as leadership research has largely failed to integrate time (in terms of content) in conceptualizations of leadership (e.g., Van der Erve, 2004), although leaders play a key role in helping the team to be temporally coordinated and achieve high levels of performance. In addition, by investigating shared temporal cognitions as a boundary condition of leadership behavior, we aim to identify a new substitute for leadership in minimizing temporal conflict in the specific context of team cognition and team functioning.

Second, our study contributes to team research in two ways: By investigating the joint effect of teams' external resources - temporal leadership - and teams' internal resources - shared temporal cognitions - we show how an implicit coordination mechanism may substitute an explicit coordination mechanism when reducing temporal conflict in teams and improving team performance. To date, no study has investigated the interplay of explicit and implicit coordination mechanisms and their joint effect on temporal conflict and team performance. Importantly, the power of shared temporal cognitions to substitute for temporal leadership also implies that both resources may compensate for the lack of the other, offering valuable implications for management and team practice. In addition, and similar to the incorporation of temporality in leadership conceptualizations, by focusing on the temporal 
Chapter 4

aspects of team functioning (shared temporal cognition and temporal conflict), we advance scholarly understanding of temporal resources and challenges for teams and follow the call for more research on a highly neglected issue in team research (Halbesleben et al., 2003; Kozlowski \& Bell, 2003).

In sum, as effective time management is one of the most challenging aspects in today's business world (e.g., Clemens \& Dalrymple, 2005), our aim to focus on time related aspects in the interplay of leadership behavior and team cognition provides researchers and practitioners with information and strategies that may help them to synchronize team members' actions, avoid conflict over temporal issues, and to attain high levels of performance.

\section{THEORETICAL BACKGROUND AND HYPOTHESES}

\section{Temporal leadership, temporal conflict, and team performance}

Temporal leadership embodies scheduling of activities, synchronization of activities, and allocation of temporal resources to task accomplishment (Mohammed \& Nadkarni, 2011). Although temporal leadership captures the task-oriented leader behaviors focused on temporality, temporal leadership is distinct from task-focused leadership (Mohammed \& Nadkarni, 2011). Task-focused leadership refers to behaviors that aim to facilitate the understanding of task requirements, the operating procedures, and the task information acquisition (Burke et al., 2006b). Those behaviors involve the standardization of task procedures, the definition of performance standards, as well as the establishment of welldefined task roles, objectives, and communication patterns (Casimir, 2001; Fleishman, 1995; Tabernero, Chambel, Curral, \& Arana, 2009). Thus, task-oriented leaders are focused on the tasks that need to be accomplished to achieve clearly defined goals and performance standards (Casimir, 2001; Burke et al., 2006b; Fleishman, 1995). In contrast, temporal leadership refers to behaviors like reminding team members of important deadlines, prioritizing tasks and allocating time to each task, and urging team members to finish subtasks on time. Thus, temporal leadership behaviors specifically refer to the temporal aspects of the task (Mohammed \& Nadkarni, 2011).

The concept of temporal leadership emerged from the need to combine temporal activities and team leadership, due to the fact that team leaders increasingly faced temporal 
challenges, such as managing multiple time frames, deciding how fast the team should act, synchronizing team members' actions, and matching the pace of the team with the environment in which it operates (Ancona et al., 2001; Halbesleben et al., 2003; Mohammed \& Nadkarni, 2011). Team leaders play a crucial role in ensuring that team members' actions are aligned and coordinated so that they can accomplish team goals on time (Murase, Carter, DeChurch, \& Marks, 2014). Team leaders not only need to provide guidance and temporal information, but they also need to ensure that all members understand when tasks should be completed, when the outputs need to be delivered to the client, and when important deadlines are set. Thus, leaders should urge members to finish subtasks on time and verify whether they are doing what they are supposed to be doing and whether they are meeting their deadlines (Gevers et al., 2006; Mohammed \& Nadkarni, 2011).

Temporal leadership has been shown to have a positive influence on team performance (Maruping, Venkatesh, Thatcher, \& Patel, 2015; Mohammed \& Nadkarni, 2011). When team leaders understand the pertinent temporal issues of their task environments, they can translate these into clear guidelines for their team members and they can develop an integrated and flexible approach for managing the team's temporal challenges and resources (Ancona et al., 2001; Mohammed \& Nadkarni, 2011). Consequently, when team leaders provide temporal guidance to their teams, they help team members to effectively perform (Ancona et al., 2001). Temporal leadership helps team members to align the rhythm of their activities with internal and external deadlines, to coordinate the timing of their actions, and to accomplish the task efficiently while meeting deadlines (Mohammed \& Nadkarni, 2011; Waller et al., 2001). Further, temporal leadership helps team members to take advantage of the positive effects of intermediate levels of time pressure because team members view time pressure as a motivator, and define their plan of action in a way that is aligned with their time constraints (Maruping et al., 2015). Thus, temporal leadership behaviors help team members to plan their work, manage their time, and achieve high levels of performance (Mohammed \& Nadkarni, 2011).

However, over the team lifecycle obstacles may arise that may hinder the team's performance. Temporal conflict is one of those obstacles. The notion of temporal conflict was recently introduced by Yang (2009) as a variation of process conflict (Mohammed, Hamilton \& Lim, 2009; Standifer et al., 2015). Process conflict refers to disagreements among team members about the way the task should be accomplished, the progress of those tasks, and who is responsible for each task (Jehn, 1997). Temporal conflict is related to the temporal 
aspects of process conflict and refers to intragroup disputes about time, the duration of a task, and the length of time the team should spend on a specific task or goal (Gevers \& Petters, 2009; Mohammed et al., 2010). In increasingly competitive organizational contexts where teams need to work together on tight deadlines over periods of weeks or months, it is particularly important to understand how temporal conflict may be avoided. Findings about temporal conflict suggest that it increases team members' frustration, leads to ambiguity about the temporal aspects of the work, and disrupts coordination. Thus, when teams are involved in conflict situations and are not temporally coordinated, team performance is negatively affected (Gevers \& Petters, 2009; Santos, Uitdewilligen, \& Passos, 2015b; Standifer et al., 2015).

Given that temporal conflict may disrupt team performance, temporal leadership behaviors that may reduce temporal conflict among team members are particularly important. The synchronizing of the team activities is likely to reduce the level of temporal conflict that teams experience because it helps to regulate the tasks' flow, to improve coordination, and to align the pace at which team members work to complete individual and team tasks (Bartel \& Milliken, 2004; Mohammed \& Nadkarni, 2011). Further, when team leaders engage in temporal behaviors they schedule the team members' activities and allocate the temporal resources of the team (Mohammed \& Nadkarni, 2011). Clear schedules and allocation of temporal resources are likely to reduce ambiguity about when tasks should be accomplished, minimize delays in meeting deadlines, and avoid disagreements about how the team should pace the task activities. Further, clear schedules and allocation of temporal resources help team members to understand how much time they should spend on each task in order to meet the deadlines (Bartel \& Milliken, 2004; Mohammed \& Nadkarni, 2011; Standifer et al., 2015).

In sum, when team leaders employ temporal behaviors, teams experience low levels of temporal conflict, thus, helping them to achieve high levels of performance.

Hypothesis 1: Temporal conflict mediates the relationship between temporal leadership and team performance.

\section{Shared temporal cognitions, temporal conflict, and team performance}

Shared temporal cognitions refer to a shared understanding among the team members of the temporal aspects of task execution (Gevers et al., 2006). Team members that 
share temporal cognitions have similar interpretations, expectations and preferences about time and deadlines, and are able to interpret temporal cues in an accurate way (Gevers et al., 2006; Standifer et al., 2015). Thus, as team members think in a similar way about the temporal aspects of work, they are able to accomplish tasks on time and achieve temporal synchronization (Bartel \& Milliken, 2004; Gevers et al., 2006).

Positive relations have been reported between teams' shared temporal cognitions and performance (Gevers et al., 2006; Mohammed \& Nadkarni, 2014). Gevers and colleagues (2006) examined whether teams that have shared temporal cognitions are better able to meet deadlines than teams that have dissimilar temporal cognitions. They found that particularly teams that work early on the task (long before the deadline), benefit from having shared temporal cognitions (Gevers et al., 2006). Recently, Mohammed and Nadkarni (2014) investigated the moderating role of shared temporal cognitions in the relationship between polychronicity diversity (i.e., a preference for engaging in several tasks at the same time; Bluedorn et al., 1999) and team performance. They found that shared temporal cognitions attenuate the negative effects of polychronicity diversity on team performance. In addition, they found a strong positive main effect of shared temporal cognitions on performance.

As described above, empirical findings have shown that temporal conflict has a negative effect on performance (Santos et al., 2015a; Standifer et al., 2015). As teams disagree about the length of time they should spend on a task and the duration of a task, ambiguity about temporal aspects emerges within the team (Gevers \& Petters, 2009). The ambiguity disrupts temporal coordination so that team members are not able to work on the task and accomplish the goals, thus, negatively affecting performance.

Similar to the proposed effect for temporal leadership, we argue that shared temporal cognitions are also able to diminish the level of temporal conflict because temporal cognitions function as temporal norms - informal rules that help teams to regulate members' behaviors related to temporal issues, such as deadlines and delays (Janicik \& Bartel, 2003). Research on team conflict shows that whereas converging norms lead to harmony, divergence in norms is likely to result in interpersonal tension and disagreement among team members (Jehn \& Mannix, 2001). When team members perceive deadlines differently they may experience delays in information exchange, which can provoke anger and frustration (Guenter, van Emmerik, \& Schreurs, 2014). In contrast, when expectations and preferences regarding temporal aspects are aligned among team members (i.e., when team members have shared temporal cognitions), 
Chapter 4

they are able to synchronize their actions and manage the available time efficiently (Janicik \& Bartel, 2003), thus, fostering high levels of team performance.

In sum, when teams share cognitions about the temporal issues they face, they will experience low levels of temporal conflict, thereby increasing their performance (Bartel \& Milliken, 2004; Gevers et al., 2006; Standifer et al., 2015).

Hypothesis 2: Temporal conflict mediates the relationship between shared temporal cognitions and team performance.

\section{Temporal leadership, shared temporal cognitions, and temporal conflict}

Substitutes for leadership theory suggests that specific individual, task and organizational characteristics may negate the leader's ability to positively or negatively influence subordinates' attitudes and effectiveness (Dionne, Yammarino, Atwater, \& James, 2002; Kerr \& Jermier, 1978). In this sense, substitutes are "aspects of the situation that cause intervening variables to be at optimal levels" (Yukl, 2010, p. 176) making leadership unneeded as it is not the only source of influence on individuals (Dionne et al., 2005). Employee characteristics that may function as a substitute for leadership include subordinate training or ability, aspects that directly influence subordinates' performance, regardless of leadership behavior (Dionne et al., 2002; Kerr \& Jermier, 1978). The idea that followers' competence and knowledge may function as a substitute for leadership has also been supported by empirical findings. Podsakoff and colleagues (1996) have shown for example that subordinates' positive perception of their ability, experience, training, and knowledge diminishes the beneficial effect of transformational leadership on role clarity. Further, De Vries, Roe, and Taillieu (2002) showed that a low need for leadership (derived from followers' evaluation of their skills and competencies) reduces the beneficial effect of leadership on followers' performance. Dionne and colleagues (2002) showed that when task variability decreases, leaders' contingent punishment is more strongly related to team effectiveness. Finally, Nübold and colleagues (2013) showed that transformational leadership behavior is not needed to promote followers' motivation and performance when followers' state core self-evaluations are high.

The idea of followers' knowledge and competence as a substitute for leadership can be easily applied to the context of temporal leadership and to teams that perform specific tasks within an environment characterized by tight deadlines and time pressure (Dionne et al., 
2002). The temporal guidance team members need for mutually adjusting their expectations and synchronizing their actions in order to experience little temporal conflict may be provided externally by temporal leadership but it may also be derived internally, from within the team itself, by the team's shared temporal cognitions. When team members already have the internal resources in the form of knowledge on effective temporal coordination, they do not need an additional external resource providing them with temporal guidance (Rico et al., 2008). In other words, shared temporal cognitions may function as a substitute for temporal leadership and may represent an alternative route to low temporal conflict and increased team performance.

Knowledge on a team's (temporal) competence that may influence leadership effectiveness may help leaders to use temporal leadership behaviors more efficiently, that is, only in situations in which teams are truly in need of it to reduce temporal conflict and enhance team performance. In the case where team members already have high shared temporal cognitions, they are not in need of the leader's temporal guidance and supervision (Dionne et al., 2005). When team members have shared temporal cognitions, this helps them to anticipate what other team members need and what they will do, which prevents friction due to unmet expectations (Guenter et al., 2014; Rico et al., 2008). Team members make sense of these temporal issues themselves and negotiate norms and rules in order to avoid misunderstandings and conflicts.

In contrast, when team members' shared temporal cognitions are weak, they may specifically benefit from the guidance of a leader with regard to temporal issues. In this case, leaders' behaviors, such as providing clear indications on how time should be spent, when deadlines are due, when meetings take place, and what is expected from the members, may be a valuable external support that diminishes a team's temporal conflicts. The leaders make sense of the pertinent issues in the team's temporal environment and provide the team members with a clear temporal structure of when team members should do what and what they can expect from each other and in their environment (Zaccaro et al., 2001).

Interestingly, as temporal leadership and shared temporal cognitions may represent different mechanisms that have the same beneficial effect on temporal conflict, they are also able to compensate for the lack of the other. When team leaders employ temporal leadership behaviors, the leaders ensure that all the members are temporally aligned, agree on the task strategies they need to follow, and effectively allocate their temporal resources to their tasks (Mohammed \& Nadkarni, 2011), thus being able to compensate for the lack of teams' shared 
Chapter 4

temporal cognitions. Conversely, shared temporal cognitions are vital to reduce the level of temporal conflict in situations of weak temporal leadership. When team leaders employ few temporal leadership behaviors, shared temporal cognitions are particularly important because they help team members to establish temporal norms. So, shared temporal cognitions work as an implicit coordination mechanism that may compensate for the lack of temporal leadership behaviors in containing temporal conflict.

In sum, temporal leadership and shared temporal cognitions function as explicit and implicit temporal coordination mechanisms that both may reduce the level of temporal conflict in teams. We propose that when teams share strong temporal cognitions, they do not need the guidance through temporal leadership in order to experience low levels of temporal conflict, thus, rendering shared temporal cognitions a substitute for leadership.

Hypothesis 3: Shared temporal cognitions moderate the relationship between temporal leadership and temporal conflict in such a way that the effect of temporal leadership on temporal conflict is weaker when shared temporal cognitions are bigh.

Finally, temporal leadership may have a differential effect on team performance via temporal conflict depending on the level of shared temporal cognitions (low vs. high). With low shared temporal cognitions, temporal leadership reduces the level of temporal conflict and, as a consequence, promotes team performance, while with high shared temporal cognitions there is no significant effect of temporal leadership on temporal conflict and, in turn, performance. The distinct effect of temporal leadership on team performance via temporal conflict depending on shared temporal cognitions again demonstrates the role of shared temporal cognitions as a substitute for temporal leadership. The research model is represented in Figure 4.1.

Hypothesis 4: The joint effect of temporal leadership and shared temporal cognitions on team performance is mediated by temporal conflict. 
Shared temporal cognitions as substitute for temporal leadership

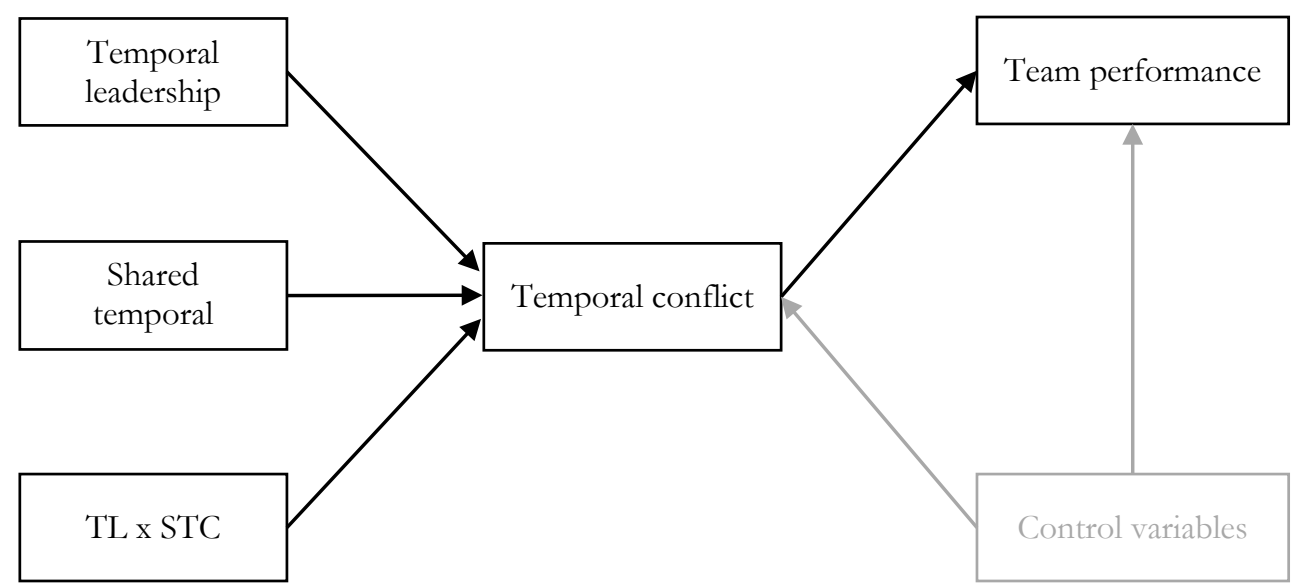

Figure 4.1. Research model. The model was tested in two separate analyses. First, we analyzed the mediating mechanism of temporal conflict in the relationship of temporal leadership (Hypothesis 1) and shared temporal cognitions (Hypothesis 2) on team performance. Second, we analyzed the moderating influence of shared temporal cognitions on the relationship between temporal leadership and team performance (Hypothesis 3), and the interaction effect of temporal leadership and shared temporal cognitions on performance via temporal conflict (Hypothesis 4). Control variables include team size, task experience, team composition (dummy variables 'students' and 'mixed'), gender, and age composition. STC = shared temporal cognitions; $\mathrm{TL}=$ temporal leadership.

\section{METHOD}

\section{Participants}

A total of 142 teams (650 individuals) participated in this study. All teams were enrolled in a national management and strategy simulation over a 5 -week period. The teams were composed of company workers $(46.0 \%)$, university students $(38.9 \%)$, or both (mixed teams) $(15.1 \%)$. Team sizes ranged from three to five members, with an average of 4.68 (SD = .59). The majority of teams consisted of five persons (74.5\%). The average age was 29 years $(S D=8.44)$ and $65.8 \%$ of the participants were male.

\section{Simulation}

Data were collected from the participants of a national management and strategy simulation. During the simulation each teams run a fictitious company, with the objective of getting the highest company share price on the simulated stock exchange. The simulation 
Chapter 4

lasted five weeks. Teams managed the company by making decisions about marketing, production, personnel, purchasing, and finance. Each team had a leader chosen by the team members when they enrolled for the simulation. The program analyzed and compared the decisions made by the competing teams and calculated the share price of each enterprise and the ranking of the teams. Then, the program produced a management report for each team, showing detailed financial results.

Time and time management were critical elements of the task the teams faced in the simulation. Participants of the simulation performed under high levels of time pressure. Every week teams needed to make decisions, analyze a large amount of information related to various areas of expertise, and coordinate their efforts efficiently to make the best decisions and submit them at a pre-scheduled moment. The simulation had very rigid weekly deadlines after which teams were not able anymore to submit their decision.

\section{Procedure}

We collected data at three different time moments. Team members answered online questionnaires through a link sent to the members by e-mail at two different time moments. Participants responded to the questionnaires in week 3 and week 4 before receiving feedback about their decisions. Shared temporal cognitions were measured in the third week of the simulation. Temporal leadership and temporal conflict were measured in the fourth week of the simulation. Performance was measured at the end of the simulation after the fifth week. Both performance and the composition variables (used as control variables) were provided by the company responsible for the simulation. Collecting data at different time moments reinforces causality inferences (Mathieu \& Taylor, 2006) and reduces common-method variance (Brannick et al., 2010; Spector, 2006).

\section{Measures}

Temporal leadership. Seven items from the study by Mohammed and Nadkarni (2011) were used and adapted for this research context. On a 7 -point scale $(1=$ never, $7=$ always $)$, participants (excluding the team leader) indicated how often the team leader demonstrated 
specific behaviors (e.g., "To what extent is your team leader effective in coordinating the team to meet goals and deadlines?"). The scale revealed good reliability $(\alpha=.92)$.

Shared temporal cognitions. Shared temporal cognitions were assessed with four items from the study by Gevers and colleagues (2006). On a 7-point scale $(1=$ totally disagree; $7=$ totally agree), the participants rated the extent to which they agreed with each sentence (e.g., "In my team, we have similar ideas about the time it takes to perform certain tasks"). The scale revealed good reliability $(\alpha=.93)$.

Temporal conflict. Temporal conflict was measured through a modified three-item scale by Yang (2009) based on the original process conflict scale developed by Jehn (1997) and Shah and Jehn (1993). On a 7-point scale $(1=$ never, $7=$ always $)$, the participants indicated how often each behavior occurred in their team: 1) "How often are there disagreements about how long to spend on specific tasks in your team?"; 2) "How often are there disagreements about time allocation in your work team (how much time to spend on tasks)?"; 3) "How often are there disagreements about how you should pace task activities in your team?". The scale revealed good reliability $(\alpha=.92)$.

Team performance. Team performance was operationalized through the share price at the end of the simulation. The share price comprehensively captures the quality of the team decisions as it is a function of the appropriateness of the teams' decisions given the specific context in which these decisions were made. Share price given in Euros, was automatically calculated and provided by the simulation and was the measure on which teams were evaluated. In the beginning of the simulation, all the companies that the teams run had the same share price: 1 Euro. The companies' share price changed over the simulation and the share price differed from one company to another, according to the teams' weekly decisions. Share price is a complex outcome measure that combines the decisions on the distinct topics on which team members need to make decisions (e.g., production, finance, and marketing) and thereby captures the combined effort of the team as a whole.

Control variables. We included team size, task experience, team composition, as well as gender and age composition of the team as control variables in our analyses. We controlled for team size (number of team members) because it can impact a team's ability to develop team 
Chapter 4

cognition, and learning processes (Bunderson \& Sutcliffe, 2003a; Wheelan, 2009). We controlled for task experience (participation in previous editions of the simulation) as this may impact team performance (Amason \& Mooney, 1999; Passos \& Caetano, 2005). We also controlled for team composition (company workers, university students, and mixed) because it may impact performance as well (Bell, Villado, Lukasik, Belau, \& Briggs, 2010; Rico, SánchezManzanares, Antino, \& Lau, 2012). The categorical variable team composition was transformed into two dummy variables ('students' and 'mixed'), using company workers as a baseline, since they were the most common teams in the competition. Finally, we also controlled for gender (male percentage within the team) and age composition (mean age of the members in a team) as it can impact performance (Apesteguia, Azmat, \& Iriberri, 2012; LePine, Hollenbeck, Ilgen, Colquitt, \& Ellis, 2002).

Confirmatory factor analysis. As shared temporal cognitions, temporal leadership, and temporal conflict all refer to temporal aspects, we conducted a first-order and a second-order confirmatory factor analysis (CFA) in Mplus using the maximum likelihood estimation method to distinguish the three constructs (Muthén \& Muthén, 2012). The first-order CFA resulted in an acceptable, although not perfect model-fit: $\chi^{2}{ }_{(62)}=467.66, p<.001$; AIC $=$ 15253.57; BIC = 15433.89; CFI = .92; SRMR = .06 (Hu \& Bentler, 1999; Schreiber et al., , 2006). A review of the modification indexes revealed the presence of residual covariance between items 1 and 4 from the temporal leadership scale. Thus, we tested a second model in which we added the residual covariance between those two items (Byrne, 2012). The second model showed a good model fit: $\chi^{2}{ }_{(61)}=218.94, p<.001$; $\mathrm{AIC}=15006.85$; $\mathrm{BIC}=15191.47$; $\mathrm{CFI}=.97$; SRMR $=.05$. The difference in the $\chi^{2}$ values between the two models was statistically significant: $\Delta \chi^{2}=248.71, \Delta d f=1, p<.001$. The second model had a good fit to the data which was better than the one of the first model. However, a review of the modification index revealed the presence of a new residual covariance between items 4 and 5 from the temporal leadership scale. Therefore, we tested a third model in which we added the residual covariance between those two items, showing a good fit to the data: $\chi^{2}{ }_{(60)}=183.44, p$ $<.001 ; \mathrm{AIC}=14973.35 ; \mathrm{BIC}=15162.26 ; \mathrm{CFI}=.98 ; \mathrm{SRMR}=.05$. The difference in the $\chi^{2}$ values between the two models again was statistically significant: $\Delta \chi^{2}=35.51, \Delta d f=1, p<$ .001. The third model fitted the data best of the three tested models.

Then, we conducted the second-order CFA constraining the variance of the secondorder factor to a value of 1.0 in order to freely estimate all second-order factor loadings 
(Byrne, 2012). The second-order model had a good model fit: $\chi^{2}{ }_{(61)}=211.43, p<.001$; AIC $=$ 14999.34; $\mathrm{BIC}=15183.96 ; \mathrm{CFI}=.97 ; \mathrm{SRMR}=.08$. The CFA results suggest that temporal leadership, shared temporal cognitions, and temporal conflict are distinct constructs.

\section{RESULTS}

\section{Aggregation}

As the level of analysis in this study was the team level, the individual answers were aggregated to the team level (Costa et al., 2013). To justify aggregation, we computed $r_{\mathrm{wg}(\mathrm{j})}$, an estimate of within-group agreement designed for multiple-item scales. The widely-applied cutoff criterion for a good estimate of $r_{\mathrm{wg}(\mathrm{j})}$ refers to a mean value equal or above 70 (James et al., 1993). However, scholars have classified this criterion as "purely arbitrary" and have argued that the major limitation of $r_{\mathrm{wg}(j)}$ is "the ambiguity in choosing the most appropriate null response pattern (i.e., distribution)" (Biemann, Cole, \& Voelpel, 2012, p. 67). Therefore, in addition to the mean values we analyzed the degree of agreement in terms of two categories (a) lack of agreement or weak agreement and b) moderate, strong, or very strong agreement) for each variable. For temporal leadership, the $r_{\mathrm{wg}(j)}$ mean value is .72, and $21.9 \%$ of the values indicate lack of agreement or weak agreement (ranging from .00 to .50), while $78.1 \%$ of the values indicate moderate, strong, or very strong agreement (ranging from .51 to 1.00) (Biemann et al., 2012). For shared temporal cognitions, the $r_{\mathrm{wg}(\mathrm{)})}$ mean value is .78, and $9.8 \%$ of the values indicate lack of agreement or weak agreement, while $90.2 \%$ indicate moderate, strong, or very strong agreement. For temporal conflict, the $r_{\mathrm{wg}(j)}$ mean value is .74, and $17.4 \%$ of the values indicate lack of agreement or weak agreement, while $82.6 \%$ indicate moderate, strong, or very strong agreement. As some teams showed lack of agreement or weak agreement, we conducted sensitivity analyses, testing our hypotheses with and without these teams in order to verify if the results have a similar pattern or are inconsistent (Biemann et al., 2012). The analyses without the teams that showed lack of agreement or weak agreement $(n=$ 115 teams) showed the same pattern of results as the analysis with those teams (i.e., the total sample; $n=142$ ). Therefore, we "can be more confident that the mixing of high and low agreement groups was not a serious enough problem” in our study (Biemann et al., 2012, p. 74). 
Chapter 4

We also computed intraclass correlation coefficients (ICC). Acceptable values of ICC(1) range between .05 and .20. To be acceptable, the values of ICC(2) need to be superior to the ones of ICC(1) (Bliese, 2000). All the values were in accordance with the required criteria for temporal leadership $\left(\operatorname{ICC}(1)=.14\right.$; $\left.\operatorname{ICC}(2)=.43 ; F_{(141,440)}=1.74, p<.001\right)$, shared temporal cognitions $\left(\operatorname{ICC}(1)=.12 ; \operatorname{ICC}(2)=.38 ; F_{(141,510)}=1.62, p<.001\right)$ and temporal conflict $\left(\operatorname{ICC}(1)=.10 ; \operatorname{ICC}(2)=.33 ; F_{(141,442)}=1.49, p<.01\right)$.

\section{Hypotheses testing}

Table 4.1. provides the means, standard deviations, and correlations for all variables at the team level. Significant positive correlations were found between temporal leadership, shared temporal cognitions, and performance. Significant negative correlations were found between temporal conflict and all the other variables. In all the analyses, task experience, team size, team composition, mean age of team members and gender composition of the team were entered as controls. 
Shared temporal cognitions as substitute for temporal leadership

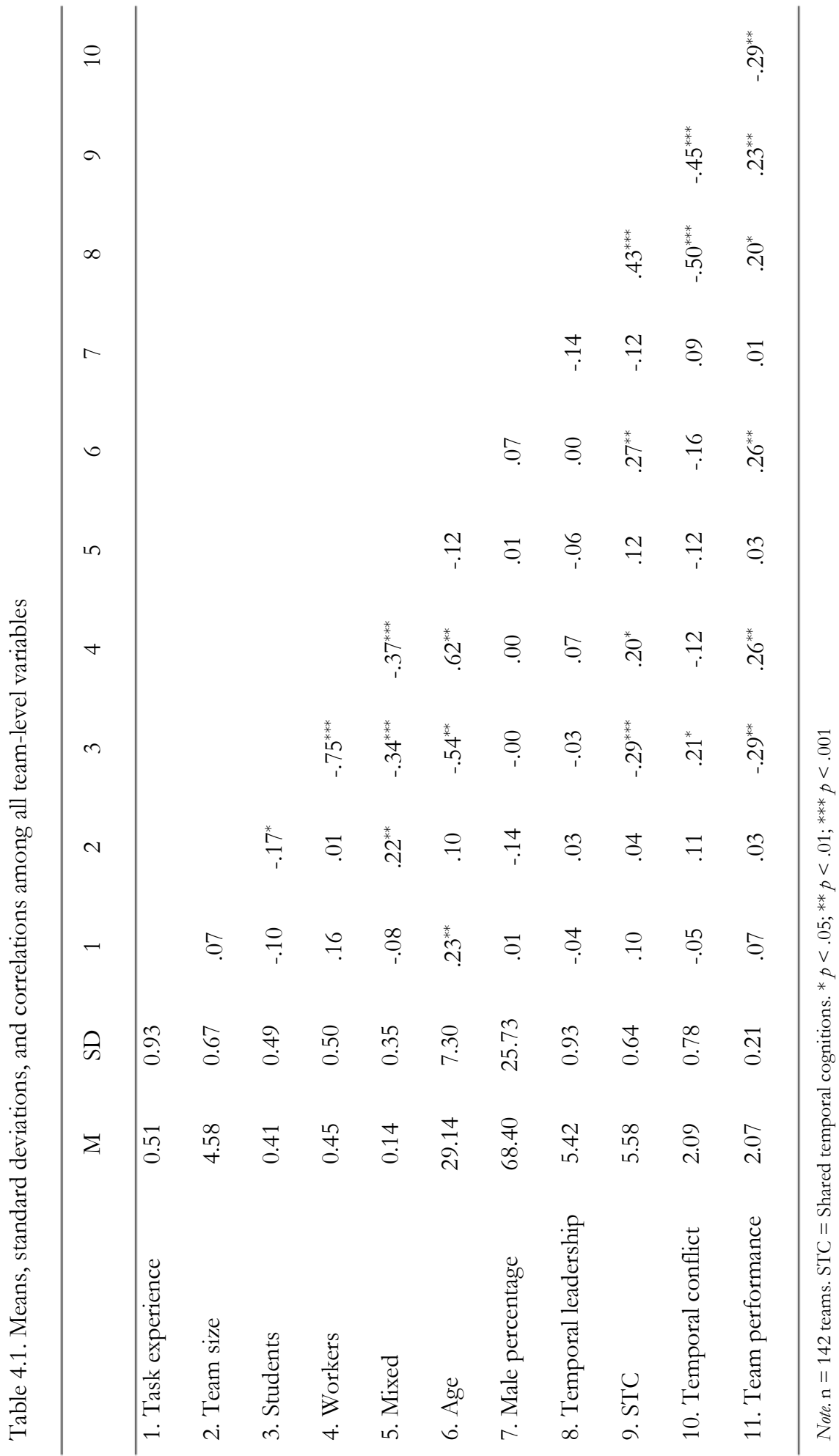


We used the statistical software Mplus (Muthén \& Muthén, 2012) for testing our hypotheses. Similar to prior studies (Nübold et al., 2013; Zhou, 2003), we used one-tailed significance tests (and 95\% confidence intervals) for all analysis because the direction of our hypotheses was specified a priori. To analyze the mediation hypotheses, we used Mplus to create 5000 bootstrap samples and use 95\% confidence intervals (CI) (Preacher \& Hayes, 2008). Hypothesis 1 proposes that temporal conflict mediates the relationship between temporal leadership and team performance. The model presented a good fit (Hu \& Bentler, 1999; Schreiber et al., 2006): $\chi^{2}{ }_{(1)}=1.25, p>.05$; CFI $=0.99$; SRMR $=.01$. The unstandardized parameter estimate showed that temporal conflict significantly mediated the relationship of temporal leadership with team performance $(.03[\mathrm{CI}=.01, .06], p<.05)$, supporting Hypothesis 1.

Hypothesis 2 proposes that temporal conflict mediates the relationship between shared temporal cognitions and team performance. The model also had a good fit: $\chi^{2}{ }_{(1)}=$ $0.54, p>.05 ; \mathrm{CFI}=1.00 ; \mathrm{SRMR}=.01$. The unstandardized parameter estimate showed that temporal conflict mediated the relationship of shared temporal cognitions and team performance $(.03[\mathrm{CI}=.01, .07], p<.05)$, supporting Hypothesis 2 .

To test the moderation analysis as well as the mediated moderation analysis in one model, we performed a unified test in Mplus creating 5000 bootstrap samples and using 95\% confidence intervals (Preacher \& Hayes, 2008). Hypothesis 3 proposes a moderating effect of shared temporal cognitions on the relationship between temporal leadership and temporal conflict. We centered the independent variables and calculated the interaction term, following the procedure suggested by Aiken and West (1991). The model presented a good fit (Hu \& Bentler, 1999; Schreiber et al., 2006): $\chi^{2}{ }_{(3)}=3.28, p>.05$; CFI $=0.99$; $\mathrm{SRMR}=.02$.

The results show that team size and the mixed teams dummy significantly predicted temporal conflict $(B=.22, p<.05 ; B=-.40, p<.05$, respectively). Regarding the main effects, temporal leadership $(B=-.32, p<.001)$ and shared temporal cognitions $(B=-.23, p<.01)$ were both significantly negatively related to temporal conflict. The interaction effect between temporal leadership and shared temporal cognitions was significantly and positively related to temporal conflict $(B=.22, p<.01)$. The results indicate that $42 \%$ of the variance of temporal conflict was explained by the control variables, the main effects of temporal leadership and shared temporal conflict, and the interaction effect between these two variables (see Table 4.2.). 
Table 4.2. Results for the interaction effect of temporal leadership and shared temporal cognitions on temporal conflict

\begin{tabular}{lccc}
\hline & \multicolumn{3}{c}{ Temporal conflict } \\
\hline Variables & $B$ & SE $B$ & $R^{2}$ \\
\hline Intercept & $2.09 * * *$ & .09 & \\
Task experience & -0.07 & .06 & \\
Team size & $0.22^{*}$ & .09 & \\
Students & 0.10 & .15 & \\
Mixed & $-0.40^{*}$ & .17 & \\
Age & -0.01 & 0.01 & \\
Male percentage & 0.00 & 0.00 & \\
Temporal leadership & $-0.32^{* * *}$ & .07 & \\
STC & $-0.23^{*}$ & .10 & .42 \\
Temporal leadership x STC & $0.22^{*}$ & .09 & \\
\hline
\end{tabular}

Note. $n=142$ teams. STC = Shared temporal cognitions. 'Students' and 'Mixed' refer to two dummy variables that were built for the categorical variable 'team composition'. 'Task experience' and 'Age' refer to team means of the respective variables. $* p<.05 ; * * * p<.001$

As the interaction effect was significant, we graphed it following the procedure suggested by Aiken and West (1991) and Dawson (2013). Figure 4.2. shows the regression slopes for the effect of high and low shared temporal cognitions on temporal conflict under strong and weak temporal leadership (+/- $1 S D$ above and below the mean, respectively; Aiken \& West, 1991). When shared temporal cognitions were low, temporal leadership was significantly related to temporal conflict $(B=-.45, p<.001)$. This means that when team members have low shared temporal cognitions, they benefit from temporal leadership in terms of experiencing low levels of temporal conflict. In contrast, in situations of strong shared temporal cognitions, temporal leadership was not significantly related to temporal conflict $(B$ $=-.18, p>.05)$. That is, teams who share strong temporal cognitions do not benefit from temporal leadership as their level of understanding of the temporal aspects in their team is already high. Thus, they were not in need of the temporal guidance of their leader. Thus, Hypothesis 3 was supported. 


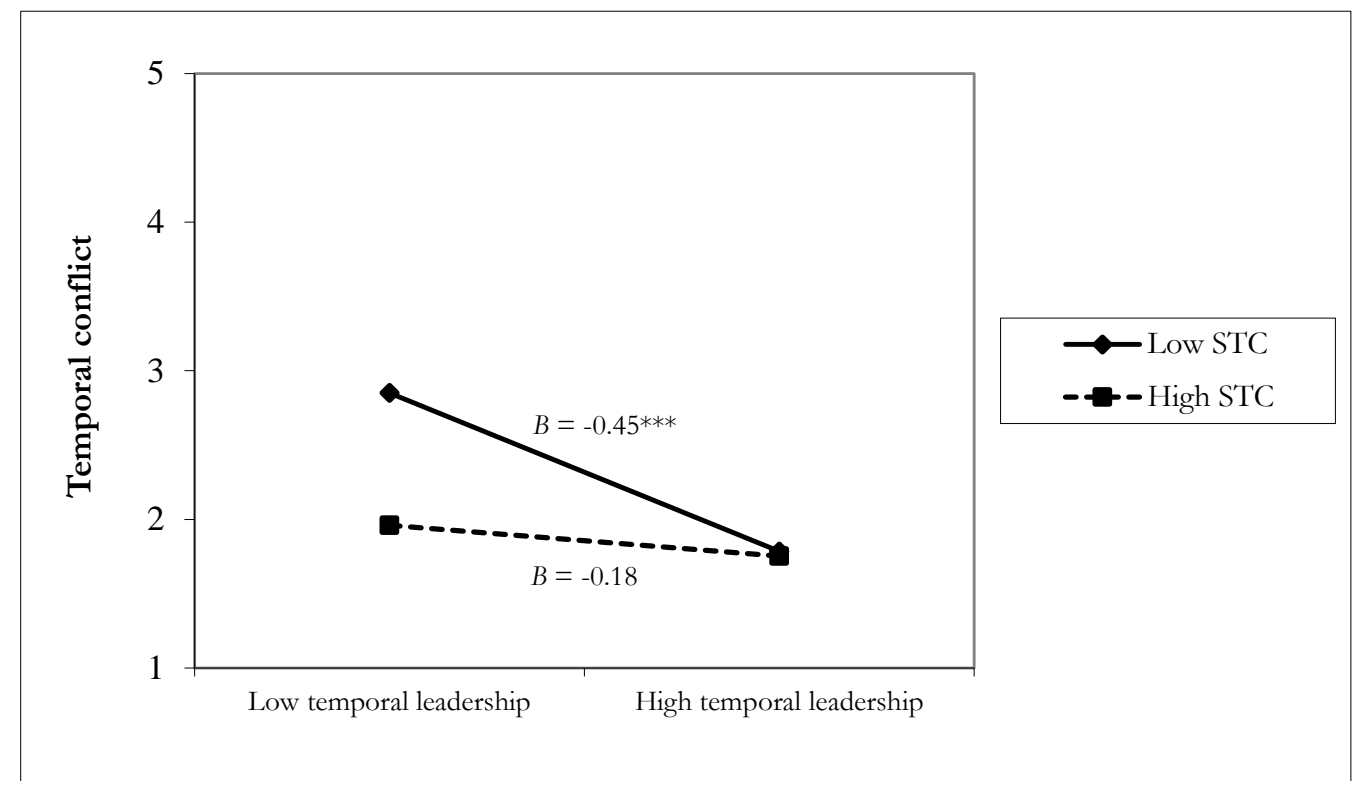

Figure 4.2. The interaction effect between shared temporal cognitions (STC) and temporal leadership on temporal conflict.

$$
\text { Note. } * * * p<.001
$$

The mediated moderation results showed that the joint effect of temporal leadership and shared temporal cognitions on team performance mediated by temporal conflict was marginally significant $(-.02[-.04, .00], p=.07)$. As the mediated moderation was significant by tendency, we further analyzed this effect by performing a multiple group analysis following the suggestion by Hox (2012). In the multiple group analysis, we tested whether the mediation effect of temporal conflict would differ between the teams that had weak shared temporal cognitions and those that had strong temporal cognitions. We created two groups for shared temporal cognitions (strong vs. weak), split on the median $(M d=5.59)$, and compared the mediation effects across these two groups (Fishbach, Friedman, \& Kruglanski, 2003; Hox, 2012; Iacobucci, Posavac, Kardes, Schneider, \& Popovich, 2015a). The results showed that for the group with weak shared temporal cognitions, temporal conflict mediated the effect of temporal leadership on performance $(.04[.01, .07], p<.01)$. In contrast, for the group with strong shared temporal cognitions, temporal conflict did not mediate the effect of temporal leadership on group performance $(-.00[-.02, .01], p>.05)$. We computed the Wald Test of Parameter Constraints to analyze if the difference between the mediation effects was significant (Hox, 2010). The results showed that the effects for the two groups significantly 
differed (Wald test $=4.47, d f=1, p<.05$ ). The use of median splits has been criticized by a number of scholars for producing Type I error and for the reduction in statistical power (McClelland, Lynch, Irwin, Spiller, \& Fitzsimons, 2015; Rucker, McShane, \& Preacher, 2015). Nevertheless, we can assume that the median split did not lead to Type I error or to misleading results because multicollinearity is absent in our data (Iacobucci et al., 2015a; Iacobucci, Posavac, Kardes, Schneider, \& Popovich, 2015b). There were no high correlations among predictor variables (i.e., the variance inflation factor (VIF) is lower than 2.5 and tolerance is higher than 0.1): Shared temporal cognitions as criterion variable: VIF $=1.35$; tolerance $=0.74$; temporal leadership as criterion variable: VIF $=1.28$; tolerance $=.78$; temporal conflict as criterion variable: VIF $=0.81$; tolerance $=1.23$ (Allison, 1999; Wooldridge, 2013). Thus, Hypothesis 4 was supported.

\section{DISCUSSION}

Our study provides two major findings that have important implications for researchers and practitioners. First, our study provides insight into the way temporal leadership and shared temporal cognitions diminish the level of temporal conflict and benefit team performance. Second, our findings suggest that shared temporal cognitions may be a substitute for temporal leadership regarding its role of reducing temporal conflict and improving team performance. High shared temporal cognitions work as an implicit coordination mechanism that helps team members to establish temporal norms, minimize temporal conflict, and achieve high levels of performance regardless of the leader's temporal behaviors. When shared temporal cognitions are low, however, temporal leadership may be an effective mean to support team members in gaining a shared understanding of temporal aspects in their team, thereby lowering temporal conflict and improving team performance.

Team leadership research has been criticized for neglecting the temporal aspects and needs of teams and organizations (Shamir, 2011; Van der Erve, 2004). Over the years, researchers have analyzed the relationship between a number of team leaders' characteristics or behaviors and outcomes (Shamir, 2011). However, researchers have failed to integrate time as content in team leadership constructs as well as to consider how the relationship between leadership inputs and outcomes are time dependent and may change over time (Shamir, 2011; Van der Erve, 2004). Although we did not account for the role of time in a longitudinal fashion (i.e., in terms of change patterns of our concepts), we incorporated temporality in 
Chapter 4

terms of content into a leadership construct (Mohammed \& Alipour, 2014). Temporal leadership is particularly relevant for team research because it considers "the process by which leaders manage multiple time frames, synchronize member contributions, and coordinate work so that deadlines are met" (Mohammed \& Alipour, 2014, p. 178). Our findings contribute to temporal leadership research showing its effect in reducing temporal conflict and promoting team performance. In organizational environments where leaders face a number of temporal challenges, such as managing multiple projects at the same time, managing people that are often member of different teams, and managing virtual and geographically dispersed teams (Murase et al., 2014; Waller et al., 2001), leaders need to employ behaviors that help the team to be temporally coordinated and to perform well.

In a similar vein, Kozlowski and Bell (2003) stated that time is "perhaps the most neglected critical issue" in team research and theory (p. 364). Over the last years, significant progress has been made on research regarding the role of time-related aspects in teams leading to the emergence of a number of temporal constructs (e.g., Gevers et al., 2006; Mohammed \& Nadkarni, 2014; Mohammed et al., 2015; Santos et al., 2015a; Standifer et al., 2015). However, researchers neither analyzed the joint effect of different temporal constructs on team processes and performance, nor investigated the mediating mechanism of a team process (temporal conflict) between temporal predictors (temporal leadership and shared temporal cognitions) and performance outcomes. Thus, our study contributes to the integration of temporality in team research by demonstrating that temporal leadership and shared temporal cognitions reduce the level of temporal conflict and promote performance. Interestingly, our findings show that shared temporal cognitions and temporal leadership may compensate for the lack of the other.

Researchers have also criticized the lack of integration of temporality in shared cognitive constructs (Mohammed et al., 2012). Nevertheless, in the last years, researchers have made efforts to integrate temporality in shared cognitive constructs and they have conceptualized and analyzed different constructs, such as shared temporal cognitions (Gevers et al., 2006), temporal transactive memory systems (Mohammed \& Nadkarni, 2014), and temporal shared mental models (Mohammed et al., 2015; Santos et al., 2015a). In the present study we focus on shared temporal cognitions analyzing the mechanisms and boundary conditions of the effects of shared temporal cognitions on performance (Bluedorn \& Jaussi, 2008; Halbesleben et al., 2003; Waller et al., 2001). Our findings suggest that temporal conflict is an important mechanism explaining how shared temporal cognitions impact team 
performance. This provides evidence that there indeed is value in continuing to examine temporal conflict in future research as it functions as a very specific mechanism explaining the effects of shared temporal cognitions on team performance.

Finally, researchers have posited that we need to conduct studies that allow us to understand "the boundary conditions under which particular aspects of team leadership affect specific mechanisms" (DeChurch, Hiller, Murase, Doty, \& Salas, 2010, p. 1081). By looking at the joint effects of temporal leadership and shared temporal cognitions on temporal conflict and team performance we heeded these calls and provide new insights on substitutes for leadership (Kerr \& Jermier, 1978). Although there has been a growing number of studies identifying individual and task level variables as potential substitutes (e.g., Dionne et al., 2002; Kerr \& Jermier, 1978; Künzle, Zala-Mezö, Kolbe, Wacker, \& Grote, 2010; Nübold et al., 2013), knowledge on team-level factors that substitute for team leadership is still lacking. Researchers have analyzed the effects of various team leader behaviors on team processes and performance (e.g., Liu, Hu, Li, Wang, \& Lin, 2014; Murase et al., 2014; Zaccaro et al., 2001); however, researchers have not presented solutions that could ensure that teams function and perform well in situations of weak or no leadership. Our findings suggest that a specific internal coordination mechanism (shared temporal cognition) may substitute a specific kind of team leadership behavior (temporal leadership behavior).

Moreover, we show that shared temporal cognitions may substitute temporal leadership in reducing the level of temporal conflict as well as promoting team performance. Our findings suggest that teams do not need temporal leadership when shared temporal cognitions are high and that both temporal leadership and shared temporal cognitions can compensate for the lack of the other. When team members have shared temporal cognitions, they make sense of the similar thoughts they have about the best way to use their time, and about the time it takes to perform the tasks to negotiate norms and rules in order to avoid misunderstandings and conflicts. Team researchers have long been interested in the interplay between explicit and implicit mechanisms of coordination (Espinosa et al., 2004). Teams may apply explicit coordination mechanisms, such as planning and leadership, or they may depend on the implicit mechanisms of shared cognition to align and integrate their activities into a unified whole (Rico et al., 2008). Although theoretical work suggests that both explicit and implicit coordination mechanisms can be important for team functioning, research that combines both types of mechanisms is lacking.

As Dionne and colleagues (2005) suggested "important substitutes for leadership can 
Chapter 4

and do exist for specific leadership behaviors without eliminating the need for all leadership behaviors" (p. 176). Shared temporal cognitions represent an effective mean to substitute for temporal leadership because both constructs focus on the temporal aspects of the task. Therefore, it seems likely that shared temporal cognitions do not function as a substitute for other leadership styles to the same extent. In addition, temporal leadership does not exclude the need for other leadership styles or functions. In fact, the combination of different behaviors or styles may be particularly beneficial for teams' functioning and performance. Both temporal and task-oriented leadership behaviors, for instance, may benefit team functioning and reduce both temporal and task conflict because leaders not only provide temporal guidance but also provide well-defined task roles, objectives and performance standards (Fleishman, 1995; Mohammed \& Nadkarni, 2011). Leader performance functions may also be beneficial for teams as they facilitate team cognitive, motivational, affective, and coordination processes and, in turn, team effectiveness (Zaccaro et al., 2001). Team leaders may employ the performance functions and suggest activities and solutions that are appropriate for the specific situation the team faces and in accordance with the deadlines and performance standards the team needs to meet.

\section{Practical implications}

Our findings present a number of implications for organizational teams and, in particular, for team leaders. The results of our study contribute to the understanding of how leaders that employ temporal behaviors help the team to avoid disagreements about the temporal aspects of work and, in turn, promote high levels of performance. Leaders should be made aware of the importance of the scheduling, synchronization and prioritization of tasks and the allocation of time for each task. Team leaders can be trained how to communicate these aspects to team members and how to monitor them over time. These temporal behaviors help the team members to prioritize tasks and allocate the time to each task, to know and agree with each other on the deadlines, when they need to start and finish a task, and how much time they need to spend on each task. If the team is temporally coordinated, it is more likely to achieve high levels of performance. Temporal agreement and temporal coordination is particularly important, for instance when teams need to work under a lot of time pressure and meet very close deadlines and when team members work at multiple projects at the same time. In those situations, whenever possible, leaders may be assigned to a 
team based on his/her ability to employ temporal behaviors.

Our findings also help to understand how the understanding about the temporal aspects that team members share is important to reduce the level of temporal conflict and foster performance. From the beginning of the team lifecycle, team members should have the opportunity to discuss with each other the best way to use their time and to allocate the time available to the tasks they need to perform. A discussion about those topics helps the team to build a temporal shared knowledge and to agree on the temporal aspects of the work which, in turn, helps the team to successfully accomplish the tasks.

Finally, our results suggest that agreement on temporal issues and team performance is not only dependent on team leaders' behaviors. At different moments, team members or team leaders may be responsible for reducing the level of temporal conflict and promoting team performance. In situations where the assigned leader has no skills or competencies to help the team to avoid temporal conflicts and achieve high levels of performance, the team members may decide to discuss with each other the best way to prioritize tasks, to finish the tasks on time, or to coordinate the team to meet deadlines. If the team leader feels that he/she is not able to help the team and that the team is able to align and integrate the activities itself, he/she can ask the team members to discuss with each other the temporal aspects of work to ensure that the team is able to accomplish its goals. Thus, team members may help each other to provide guidance and temporal information and to verify whether the team is doing what it is supposed to be doing. In sum, strong shared temporal cognitions may compensate for the lack of high temporal leadership behaviors.

\section{Limitations and directions for future research}

In the present study we focused on time as the content of several concepts. Although we stressed the added value our study brings for team and team leadership research, future studies should also focus on time as a dynamic process (i.e., in longitudinal studies) in order to understand the role of time on the different phenomena. It could be analyzed, for instance, whether the effect of temporal leadership on temporal conflict and performance depends on the specific phase the team is in. Future studies should combine the concept of time as integral part of the study concepts and time as a dynamic process inherent in the study design. Longitudinal designs should be used to track the changes in team functioning over the team lifecycle. Further, future research should explore questions about time in teams; particularly in 
Chapter 4

teams for which time (pressure) is an essential building block for their emergence, such as rapid response or crisis management teams.

Our measurement at both the individual and team level showed some limitations. At the individual level, our data revealed the presence of residual covariance between items from the leadership scale, which refers to a measurement error in item responses (Byrne, 2012). Residual covariance may be related to social desirability of respondents or to a very high overlap in item content (Byrne, 2012). Future research should ensure that social desirability becomes less problematic, for instance, by increasing the motivation to provide honest responses.

At the team level, our data revealed that for about $10 \%-20 \%$ of the teams (depending on the variable) the $r_{\mathrm{wg}(\mathrm{j})}$ were poor $(>.50)$, indicating a lack of or weak agreement among team members. Nevertheless, most of the teams had moderate, strong, or very strong agreement, and the analyses without the teams that had a lack of agreement or weak agreement showed the same pattern of results as the analysis with those teams. Therefore, we recommend to adopt a similar approach in future research and conduct sensitivity analyses when the results reveal $r_{\mathrm{wg}(\mathfrak{)})}$ with lack of or weak agreement. In this way, hypotheses can be tested with and without those teams that lack or only show weak agreement to verify whether the results have a similar or different pattern. Regarding the temporal leadership measure, it could be interesting for future research to analyze the dispersion in the evaluation team members make about their leader's temporal leadership behaviors (Boies \& Howell, 2006; Graen \& Uhl-Bien, 1995). According to LMX theory, "leaders do not treat all followers identically; rather, they develop different quality of relationships with followers" (Boies \& Howell, 2006, p. 246). Thus, team leaders may employ different temporal behaviors depending on characteristics of the team members. For instance, leaders may more actively engage in temporal leadership towards members they perceive to need more temporal guidance.

Further, future research should analyze the consequences and implications for those teams that have low agreement in these constructs. Team researchers have predominantly focused on similar perceptions team members have about team dynamics phenomena and have operationalized these in terms of the mean scores on these variables among the team members. However, multilevel theorists argue that dispersion is an inherent property of any group-level phenomenon and scholars should go beyond operationalization of mean levels and also include dispersion indices in order to fully understand how these phenomena impact team outcomes (De Jong, Van der Vegt, \& Molleman, 2007; Kozlowski \& Klein, 2000; 
Kozlowski \& Chao, 2012).

Despite our approach to separate the different measurements in time, commonmethod variance may be a limitation (Siemsen, Roth, \& Oliveira, 2010). Some authors argue that common-method variance is not a problem and that the spurious causes of relationships among variables are related to the mixture of methods and constructs and not related to the methods themselves (Brannick et al., 2010). However, in order to limit concerns for commonmethod variance, in particular because we use perceptual measures answered by the same respondents, we measured the variables at different moments in time.

\section{CONCLUSION}

In increasingly complex, dynamic and time pressed organizational contexts, team leaders have a crucial role to play in providing team members with important tools to help them achieve high levels of performance within strict deadlines. Our study shows that team leader behaviors that focus on the temporal aspects of work help team members to reduce temporal conflict and achieve high levels of performance. Further, our findings suggest that team members' shared temporal cognitions function as a substitute for temporal leadership. That is, if shared temporal cognitions are high, there is no need for temporal leadership in order for teams to experience low levels of temporal conflict. This also implies that shared temporal cognitions can compensate for the lack of temporal leadership and vice versa in order to diminish temporal conflict in teams. 



\section{CHAPTER $5^{6}$}

\section{WHEN SHARED COGNITION LEADS TO CLOSED MINDS: TEMPORAL MENTAL MODELS, TEAM LEARNING, ADAPTATION AND PERFORMANCE}

${ }^{6}$ This chapter is published as:

Santos, C.M., Passos, A.M., \& Uitdewilligen, S. (2016). When shared cognition leads to closed minds: Temporal mental models, team learning, adaptation and performance. European Management Journal, 34, 258-268. DOI: 10.1016/j.emj.2015.11.006.

http://www.sciencedirect.com/science/article/pii/S0263237315001061 
Chapter 5

\begin{abstract}
In this study we examined the moderating effect of temporal mental model accuracy on the relationship between temporal mental model similarity and team learning. Further, we investigated the mediating mechanism of team adaptation in the relationship between team learning and performance. The study was conducted in a management simulation involving 68 teams (319 individuals). We collected data at three time points. The results showed that when accuracy is high, temporal mental model similarity is not significantly related to team learning; whereas, when accuracy is low, the more similar the team members' mental models are, the less they engage in learning behaviors. This suggests that sharing an inaccurate mental model leads to closed minds. In addition, we found team adaptation to mediate the relationship between team learning and performance. These findings emphasize the importance of temporal mental models in predicting team learning, and the importance of team learning for team adaptation and performance.
\end{abstract}


When shared cognition leads to closed minds

\section{INTRODUCTION}

Increasingly scholars emphasize that in complex and demanding contexts teams need to be able to adapt quickly and appropriately to recurrent changes (Baard et al., 2014; Burke et al., 2006a; Rosen et al., 2011). Teams need to adjust their cognitive and behavioral processes to allow them to evaluate and analyze situations in order to adjust to them in the best way possible (Burke et al., 2006a; Randall, Resick, \& DeChurch, 2011; Uitdewilligen et al., 2013). Team learning plays a crucial role as an essential, though not sufficient, condition for team adaptation (Burke et al., 2006; Rosen et al., 2011). Team adaptation, as a process, occurs when a team recognizes that a change happens in the team environment, and is able to effectively address the unexpected situation (Baard et al., 2014; Maynard et al., 2015). When team members engage in team learning processes, they evaluate and reflect on past performance episodes and interpret the consequences of team actions. Therefore, they are likely to improve their task and team processes, which enables the team to adapt to novel situations, which in turn facilitates performance - the objective criterion that indicates team level task accomplishment (Hackman, 1987; Rosen et al., 2011).

Team learning refers to a team process in which team members ask questions, seek feedback, reflect and discuss results, errors, and (un)expected outcomes (Edmondson, 1999). A shared understanding about the temporal aspects of work is crucial to promote the team learning process. As teams operate in organizational contexts that are systematically pressured by time, they are better able to engage in learning behaviors when team members share a temporal mental model - common knowledge about deadlines for task accomplishment, the pacing or speed at which activities occur, the time available for each activity, and the sequencing of tasks (Mohammed et al., 2015; Santos, Uitdewilligen, \& Passos, 2015a; Standifer \& Bluedorn, 2006). A temporal mental model helps teams to coordinate their activities according to the time schedule and to anticipate and understand the actions of each other based on a commonly shared blueprint of plans and schedules (Mohammed et al., 2015; Santos et al., 2015a).

In this study we focus on the relevance of temporal mental models for team learning. We postulate that when team members share a temporal mental model they make an efficient use of the team's time, thereby creating more time for the team to engage in learning behaviors (Santos et al., 2015a). The common temporal understanding ensures that team members are aligned regarding the temporal demands of the team's work, such as when 
Chapter 5

deadlines have to be met and how much time is available for each activity (Cannon-Bowers et al., 1993). Teams may have a similar temporal mental model - a mental model that is similar among team members - and an accurate temporal mental model - a mental model that is appropriate for the task according to experts in the respective area (Edwards et al., 2006). Thus far, researchers have investigated how task and team mental model similarity and accuracy interact to predict team adaptation and performance (e.g., Burtscher et al., 2011; Marks et al., 2000); however, research on the interactive effects of temporal mental model similarity and accuracy is missing. Moreover, a relevant discussion that needs clarification is whether teams with a similar but inaccurate temporal mental model are able to learn from each other as much as teams in which team members share a similar and accurate temporal mental model. We posit that when team members have a similar but inaccurate understanding of the temporal aspects of their work, this will keep them from discussing their tasks, reflecting on the results and learning from each other.

To summarize, with this study we contribute to shared mental model literature by analyzing the temporal dimension of mental models, and analyzing the interactive effect of temporal mental model similarity and accuracy on team learning. Further, we contribute to the team learning literature by analyzing the effect of team learning on team adaptation, and on performance, as recent theoretical models have suggested (Burke et al., 2006; Rosen et al., 2011).

\section{THEORETICAL BACKGROUND AND HYPOTHESES}

\section{Team learning and temporal mental models}

In 2005, the United States faced Hurricane Katrina - one of the most deadly hurricanes in the United States' history (Moynihan, 2007). While Katrina raged through the United States, the different teams that worked to save people and to minimize the damages failed to coordinate themselves, to learn from each other, and to adapt their responses to the unexpected situation and under a lot of time pressure. Additionally, there were a number of delays in making the correct decisions, which led to dramatic consequences: aid was not delivered in time, people were not evacuated in time because of the delays in providing buses to do it, and people were left with no basic supplies (Moynihan, 2007). This example demonstrates the negative consequences that may occur when teams fail mostly because team 
members do not establish and maintain congruence in their temporal perceptions (Mohammed et al., 2012). As Moynihan (2007) mentioned, "time is an essential ingredient in learning", and, although learning has occurred during the Hurricane, "learning did not occur rapidly enough to dramatically impact the Katrina response" (p. 18).

The example of how teams dealt with Hurricane Katrina shows the importance of congruence in team members' temporal perceptions in extreme conditions, as well as the importance of engaging in team learning behaviors. These two aspects are not only important in extreme situations like the Hurricane Katrina. Most of the team work environments are increasingly complex, dynamic, and adaptive, and teams are constantly pressured by time. Nowadays, people often are member of more than one team, and team members may be geographically dispersed and often need to manage multiple projects simultaneously (Ancona \& Chong, 1999; Waller et al., 2001). Therefore, teams often need to discuss, make decisions, and achieve their goals in a short-period of time and under high time pressure (Waller et al., 2001). Team members planning and setting deadlines is crucial to ensure that teams are able to perform their tasks under time pressure and stress, particularly when something unexpected happens. In sum, because managing time well is so crucial for team functioning, it is important that team members develop a shared and accurate cognitive structure about the temporal aspects of their work.

We analyze the effect of temporal mental model on team learning, arguing that a mental model works as a common basis that provides a fertile breeding ground for teams to engage in team learning behaviors (Santos et al., 2015a; Tindale et al., 2008), which is related to the resource allocation perspective on team functioning (Barnes et al., 2008; Kanfer \& Ackerman, 1989). Team learning "is a resource-intensive process that detracts from core task performance and that consumes time and cognitive resources" (Santos et al., 2015a, p. 719). Therefore, teams in which members have a similar and accurate temporal mental model are more likely to naturally engage in team learning behaviors without requiring any substantial extra effort in the process. When team members have a common understanding about the temporal aspects of the work, they are able to communicate ideas and coordinate themselves. Thus, team members are able to engage in team learning behaviors using few temporal and cognitive resources (Bunderson \& Sutcliffe, 2003a).

A temporal mental model helps teams to coordinate their actions and perform the tasks on time, and is particularly important when team members are highly interdependent, and need to collaborate and share information continuously (Mohammed et al., 2015). 
Chapter 5

Further, a temporal mental model allows team members to anticipate and understand how the actions of the other team members fit within the plans and schedules, and to know in advance what other team members need to finish a task on time (Gevers et al., 2006; Standifer \& Bluedorn, 2006). A similar temporal mental model helps team members to synchronize their actions with the actions of other members; while an accurate temporal mental model helps to fit the team' actions with the external temporal demands (Gevers et al., 2006; Mohammed et al., 2012, 2015).

A number of studies have integrated the notion of time in team cognition and team processes research (e.g., Gevers et al., 2006; Standifer et al., 2015). Moreover, several authors argued that shared mental models should cover not only task and team contents but also the temporal aspects of work (Guiette \& Vandenbempt, 2013; Mohammed et al., 2012; Standifer \& Bluedorn, 2006). However, to date there are only two studies (Mohammed et al., 2015; Santos et al., 2015a) measuring temporal mental models. Mohammed and colleagues' study (2015) operationalized the notion of temporal mental model assessing its discriminant validity relative to team and task mental model constructs in predicting team performance. The results showed that temporal mental model positively predicted team performance beyond task and team mental models. The results also showed that temporal mental model positively influenced team performance early on and later on in the teams' lifecycle. Santos and colleagues' study (2015) investigated whether team learning processes lead to performance improvement, and whether this relationship is moderated by the similarity of shared mental models. The authors looked at the effects of task, team, and temporal mental models. Their results showed that when task and temporal mental model similarity were high, team learning processes were positively related to team performance improvement. Thus, temporal and task mental models function as a boundary condition for the translation of team learning processes into team performance improvement (Santos et al., 2015).

\section{The effect of temporal mental model similarity and accuracy on team learning}

Team members that have a similar temporal mental model have a common vision regarding important temporal aspects of work (Mohammed et al., 2015; Santos et al., 2015a). When team members have a similar understanding of the timing of their processes, they use the team's time in an effective way, which creates more time for the team to engage in learning behaviors. With a temporal mental model, team members are able to time and synchronize 
their actions and activities, and to be aware about the time they have for learning behaviors in order to not interfere with other aspects of task execution (Bunderson \& Sutcliffe, 2003a; March, 1991). Further, teams that have a similar temporal mental model are likely to coordinate their activities, increase the efficiency of their communication, and decrease communication and coordination breakdowns, during the team learning process, leading them to engage in team learning behaviors (Cataldo \& Herbsleb, 2013). The reduction in communication and coordination breakdowns lead to the team to engage in team learning behaviors because team members are able to efficiently communicate errors, discuss them, analyze the information and find solutions for the problems. Further, as team members are coordinated, the suggestions, feedback and ideas are voiced at appropriate times being more likely to be acquired and accepted by other team members (Kulik \& Kulik, 1988).

Team members need to have similar knowledge about the deadlines, the pacing at which activities occur, and the time available for each activity in order to engage in team learning behaviors (Gevers et al., 2006; Standifer \& Bluedorn, 2006). By sharing this temporal understanding, team members are able to more efficiently learn from each other, seek feedback, and reflect on results. Furthermore, when they have similar mental models, the ideas team members discuss, the feedback they receive and provide, and the changes they plan will be aligned with the team plans and deadlines (Gevers et al., 2006). Without a similar temporal mental model, team members may answer questions in a way that is not related to the tasks they need to accomplish, may seek or give inappropriate feedback, or even work and discuss ideas and plans that take into account different deadlines and schedules (Tindale et al., 2008).

\section{Hypothesis 1 - Temporal mental model similarity is positively related to team learning.}

When team members have an accurate temporal mental model, the mental model is an appropriate representation of the temporal aspects of the teams' work (Marks et al., 2000). This means that they have a correct understanding about the right priorities, the appropriate strategies to accomplish the tasks on time, and the correct amount of time they have for each activity. Teams that have an accurate temporal mental model are likely to effectively discuss the tasks, provide feedback, and exchange ideas. They are able to optimize the timing and synchronization of team learning. Those teams are likely to engage in team learning behaviors without interfering with task accomplishment because they are correct about the most appropriate time to work on the tasks, when the deadlines need to be met, the exact time they 
have to discuss, and when they need to stop learn from each other and return to their tasks in order to accomplish the team goals on time (Edmondson, 1999; Santos et al., 2015a). If team members have an inaccurate temporal mental model, team members may focus on the wrong priorities and underestimate (or overestimate) the time they have to accomplish the tasks and meet deadlines. Therefore, team members may not engage in team learning behaviors when, in fact, they had time to do it, or may engage in team learning behaviors but discuss issues very quickly, or may engage in inappropriate learning behaviors to the team's task and goal.

\section{Hypothesis 2 - Temporal mental model accuracy is positively related to team learning.}

Temporal mental model similarity and accuracy also have an interaction effect on team learning. Although we expect that overall temporal mental model similarity is positively related to team learning behaviors, if the team members have an inaccurate temporal mental model, team members will engage less in team learning behaviors. It is not necessarily detrimental for team learning if members initially have dissimilar inaccurate temporal mental models. If members disagree on temporal aspects of their task, this can serve as an impetus for discussions and clarifications, resulting in reflection and team learning behaviors (Van den Boosche, Gijselaers, Segers, Woltjer, \& Kirschner, 2011). However, if team members agree on an inaccurate mental model this may lead to closed-mindedness where team members refrain from engaging in learning behaviors because they incorrectly perceive that they already have an accurate mental model. Similarity of mental models may foster rigidity as team members may resist letting go of previously established beliefs when these are reinforced by others (Gersick \& Hackman, 1990).

Closed-mindedness occurs when team members engage in collective rationalization ignoring or resisting to new information and ideas in particular when those ideas are inconsistent with the team's beliefs or challenge the existing ideas (Dijksterhuis et al., 1996; Thompson, 2004). Closed-mindedness is a symptom of groupthink (Janis, 1972) - a phenomenon that "involves a deterioration of mental efficiency, reality testing, and moral judgements as a result of group pressures toward conformity of opinion" (Thompson, 2004, p. 130). Teams that have a similar and inaccurate temporal mental model may fail to retest, question, or discuss the ideas and assumptions they have about the way they work. Team members' mental models may reach a level of overlap that hinders the team to engage in team learning behaviors because "an exact replication [of mental models] would reduce the 
availability of alternative solutions or strategies because of team members' varying perspectives and understandings" (Salas et al., 2005, p. 566). In sum, when teams have inaccurate temporal mental models, they will engage in less learning behaviors when mental models are similar than when they are dissimilar.

Hypothesis 3 - The influence of temporal mental model similarity on team learning depends on temporal mental model accuracy; the less accurate the mental model is, the weaker the relationship between mental model similarity and team learning will be.

\section{Team learning, adaptation, and performance}

A number of studies have demonstrated that team learning has a positive effect on team performance because by asking questions, discussing errors, and seeking feedback, team members can test their assumptions about the way they work, discuss divergent opinions, and thereby achieve high levels of performance (Edmondson, 1999; Savelsbergh et al., 2009; van Woerkom \& Croon, 2009). Teams also need to be able to adapt quickly to recurrent changes in order to perform well (Burke et al., 2006a; Uitdewilligen et al., 2013). Often changes occurring in team contexts are unexpected. Team learning behaviors help teams to adjust their interaction processes, and to evaluate and analyze the changing situations (Rosen et al., 2011; Uitdewilligen et al., 2013). Through the team learning process, team members evaluate past performance episodes, interpret the consequences of team actions, explore different perspectives, and proactively develop new strategies (Burke et al., 2006a; Kozlowski \& Bell, 2008). Those learning behaviors facilitate teams in changing and improving their working methods, which is an important requirement for team adaptation (Kozlowski \& Bell, 2008; Rosen et al., 2011). Thus, when teams engage in team learning behaviors they acquire, combine, and share knowledge that allows them to work in a coordinated way, and to behave adaptively (Rosen et al., 2001). As adaptation is crucial for performance, especially in dynamic contexts, teams that behave adaptively achieve high levels of performance because they adapt the way they work, use new ideas to deal with problems, and think about alternative solutions in short periods of time (Marques-Quinteiro et al., 2013; Maynard et al., 2015). So, teams that effectively engage in team learning behaviors are able to exchange information and ideas about the way they perform in previous task episodes, and integrate new with previous information 
to overcome the obstacles. They are able to adapt the methods they use according to the unexpected situations they face. Then, they achieve high levels of performance.

Hypothesis 4 - Team adaptation mediates the relationship between team learning and team performance.

The research model is represented in Figure 5.1.

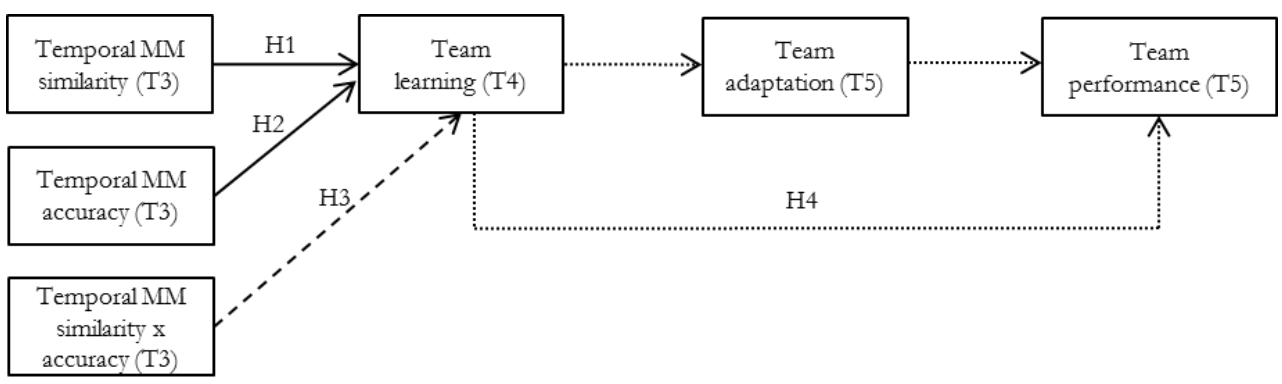

Figure 5.1. Research model and hypotheses. Solid arrows represent direct effects (H1 and H2). Dashed arrow represents the moderation effect $(\mathrm{H} 3)$. Dotted arrow represents the mediation effect $(\mathrm{H} 4)$. $\mathrm{T}=$ Time. $\mathrm{MM}=\mathrm{Mental}$ model.

\section{METHOD}

\section{Participants}

A total of 68 teams (319 individuals) participated in this study. All teams were enrolled in a national management and strategy simulation for a 5-week period. The teams were composed of workers (45.1\%), or university students $(36.4 \%)$, or a mix of workers and students $(18.5 \%)$. The teams consisted of three to five persons with an average team size of 4.76 persons $($ S.D. $=0.51)$. The average age of the members was 31 years $($ S.D. $=8.88)$ and 66.5 percent of the members were male.

Worker teams' participants had a degree in engineering (38.2\%), finance, economics or accounting $(26.4 \%)$, management $(20.1 \%)$, or other (15.3\%). Participants from mixed teams had a degree in management (27.5\%), finance, economics, and accounting (26.1\%), engineering $(26.1 \%)$, or other $(14.3 \%) ; 5.8 \%$ of participants did not provide information about their degree. University students were completing their bachelor or master degree in 
management $(57.0 \%)$, engineering $(20.7 \%)$, finance, economics or accounting $(12.4 \%)$, or other $(9.9 \%)$.

\section{Simulation}

Data were collected from the participants of the Global Management Challenge ${ }^{\circledR}$ (GMC®) developed by a company specialized in developing business simulations (http://www.worldgmc.com). The GMC ${ }^{\circledR}$ consists of a management and strategy simulation in which each team runs a company, with the objective of getting the highest company share price on the simulated stock exchange. Most of the teams are sponsored by their employer organization or by large organizations that sponsor GMC® within their scientific and cultural patronage policy. In fact, many top companies encourage their employees to take part in it. Companies that sponsor teams formed by their own employees use the simulation as a training experience to promote the development of employees' skills about management, decision making in changing and complex environments, and teamwork. Students use the simulation as a way to acquire skills and competencies of business operations related to running a company (e.g., marketing, human resources, and production). As GMC® participants needed to apply for take part of the simulation, and they were free to assemble their own team, some team members may know each other beforehand from their university (for student teams), or from their jobs (worker teams).

An incentive is offered in the simulation. The winning team of the national final of the GMC® wins an Intercontinental trip for each team member and represents the country in the international final of the simulation against the winning teams of the other countries. Similar simulations have been used by others researchers (e.g., Costa et al., 2014; MarquesQuinteiro, Passos, \& Curral, 2014; Mathieu \& Rapp, 2009; Santos \& Passos, 2013).

Teams were organized into groups (each group with a maximum of 8 teams). Each group comprised a competitive market, in which the teams had to compete with one another in a common business environment (the "group") to achieve the highest share price. Teams managed the company by making decisions, once a week over five weeks, about marketing, production, human resources, purchasing, and finances. A simulator analyzed and compared the decisions made by the various competing teams, and calculated the share price of each enterprise and the ranking of all teams after each team made their decision. Then each team received feedback about their decisions through a management report the simulator produced 
Chapter 5

showing the detailed results in financial and operational terms. The competition simulates a year and a quarter of each company's activity, and each week corresponds to one quarter of year.

Team adaptation is important in this context because teams need to adapt their strategy in accordance with their performance and other teams' performance because they compete with one another to achieve the highest share price. Further, over the simulation unexpected events can occur in companies that teams manage, such as, strikes and absenteeism, or even world events, such as, wars and physical disasters. Thus, teams need to develop the ability to adapt after these unexpected events occur.

\section{Procedure}

Team members answered three different on-line questionnaires during the simulation. The link to the questionnaires was sent to the team members by e-mail at different weeks of the simulation, two days after the beginning of each weekly task. A reminder was sent to the participants one day before the deadline to submit the weekly decision. The questionnaires were available until the moment in which teams had to submit their weekly decision. Participants answered to the questionnaires in week 3, week 4 and week 5 before receiving the management report. Temporal mental model was measured in the third week of the simulation. Team learning was measured in the fourth week of the simulation. Team adaptation and performance were measured at the end of the simulation after the fifth week. Performance and composition variables were provided by the company responsible for the simulation. This longitudinal procedure reinforces causality inferences (Mathieu \& Taylor, 2006) and reduces the common-method variance (Brannick et al., 2010; Spector, 2006).

\section{Measures}

Temporal mental model. To operationalize the temporal mental model, we created four items for the specific context. By means of a detailed task analysis of the simulation and with the help of a group of company managers who develop the simulation, we derived four sentences for understanding the temporal aspects of the simulation (Mathieu \& Rapp, 2009; Uitdewilligen et al., 2013). Then those four sentences were paired among each other resulting in six pairs of sentences. A list of pairs of sentences is provided in the Appendix C. We asked 
each team member to rate the relatedness of the pairs of statements on a 7-point scale $(1=$ the sentences are not related to $7=$ the sentences are extremely related).

Shared mental models may be measured through different techniques: content (i.e., the focus of mental models), elicitation of content (i.e., the content or components of the mental model), structure representation or structural networks (i.e., the way as the content of mental models is cognitively organized), and representation of emergence (i.e., the representation of the team-mental model as a collection of individuals' mental models) (Mohammed et al., 2000). The procedure we used to operationalize the temporal mental model - structural representation using pairwise ratings - is used most generally and considered most valid in mental models research (Resick et al., 2010b). Consistent with Lim and Klein (2006), we asked participants to evaluate the relatedness between pairs of brief sentences. This pairwise rating procedure is a way to analyze the network of relationships among key temporal aspects of the simulation associated with the achievement of the team goal (Resick et al., 2010b).

Temporal mental model similarity. To calculate temporal mental model similarity, we used UCINET (Borgatti et al., 2002), following the procedure developed by Mathieu and colleagues (2000). This network-analysis program provides a similarity measure based on Pearson's correlations. As each team member evaluated six pairs of sentences, the first step was to make a matrix for each team containing the individuals' matrices (i.e., the individuals' evaluation of the pairs of sentences). Next, we used UCINET to calculate the similarity among the six pairs of sentences of all team members, for each team-level matrix. This similarity index ranged from -1 (complete disagreement) to 1 (complete agreement/sharedness). The six similarity values were also displayed in a matrix. The mental model similarity index of each team was then calculated based on the average of the six similarity values.

Temporal mental model accuracy. To obtain an assessment of mental model accuracy, we asked 12 expert-members (members who had won previous editions of the simulation) to answer to the same mental models items. To analyze the reliability of our mental model accuracy measure we computed the intraclass correlation coefficient (ICC) (Rankin \& Stokes, 1998). The estimated reliability score across raters is 0.85 with a $95 \%$ confidence interval of CI lower bound: $0.70, \mathrm{CI}$ upper bound: 0.94 (Rankin \& Stokes, 1998), supporting the reliability of the measure. We calculated individual mental model accuracy for each item by calculating the 
Chapter 5

absolute difference between each team member's response and the average response value obtained by the experts (Webber et al., 2000). To calculate the levels of mental model accuracy for each team, we averaged the individual team member mental model accuracy scores. The mental model accuracy index ranges from 0 (completely accurate mental models) to 6 (completely inaccurate mental models). Values were recoded in a way that the lowest values corresponded to more inaccurate mental models and the highest values corresponded to more accurate mental models.

Team learning. Team learning was measured using the 15 items of Savelsbergh and colleagues (2009) that cover the following dimensions: co-construction of meaning, exploring different perspectives, error analysis, and error communication. On a 7 -point scale $(1=$ totally disagree; 7 totally agree), the participants rated the extent to which they agree with each sentence (e.g., "Team members collectively draw conclusions from the ideas that are discussed in the team"). A confirmatory factor analysis (CFA) was implemented by Mplus (Muthén \& Muthén, 2012) which presented a goodness-of-fit index as all indices fell within acceptable ranges (Hu $\&$ Bentler, 1999; Schreiber et al., 2006): $\chi^{2}{ }_{(91)}=1574.34, p=.00$; RMSEA $=.00$; CFI $=.94$; TLI $=.93 ;$ SRMR $=.03$. The internal consistency of the scale was very good $(\alpha=.98)$.

Team adaptation. Ten items adapted from the study by Pulakos and colleagues (2002) were used to measure team adaptation (Marques-Quinteiro et al., 2013). On a 7-point scale (1 = totally disagree to $7=$ totally agree), the participants rated the extent to which they agreed with each sentence (e.g., "My team was effective in quickly developing plans of action for dealing with unpredictable situations"). The internal consistency of the scale was very good $(\alpha=.98)$.

As team learning and team adaptation were highly correlated, we conducted a CFA in Mplus to distinguish the two constructs (Muthén \& Muthén, 2012). In the first model all variables were modeled as indicators of a single factor: $\chi^{2}{ }_{(252)}=1249.55, p=.00$; AIC $=$ 1727.19; $\mathrm{BIC}=1890.10 ; \mathrm{RMSEA}=.00 ; \mathrm{CFI}=.66 ; \mathrm{TLI}=.63 ; \mathrm{SRMR}=.11$. In the second model team learning was modeled as a single factor, and team adaptation was modeled as other single factor: $\chi^{2}{ }_{(251)}=539.80, p=.00$; $\mathrm{AIC}=1019.44$; $\mathrm{BIC}=1184.62$; $\mathrm{RMSEA}=.00$; $\mathrm{CFI}=.90 ; \mathrm{TLI}=.89 ; \mathrm{SRMR}=.04$. Although the fit of the second model was not perfect, because of the CFI and TLI values that were not above .95 (Schreiber et al., 2006), it 
presented a better goodness of fit than the first model suggesting that team learning and team adaptation are distinct constructs (Hu \& Bentler, 1999).

Team performance. Team performance was operationalized as the share price at the end of the simulation. The share price comprehensively captured the quality of the team decisions, as it was a function of the appropriateness of the teams' decisions given the specific context in which these decisions were made. Share price was given in Euros, was automatically calculated by the simulation, and was the measure on which teams were evaluated. Share price was a complex outcome measure that combines the decisions on the distinct topics on which team members need to make decisions (e.g., production, finance, and marketing), and thereby captured the combined effort of the team as a whole.

Control variables. We included team size, task experience, team familiarity, team composition (workers, university students, and both), and the group in which teams compete as control variables in our analyses (Humphrey et al., 2009; van Knippenberg \& Schippers, 2007). We controlled for team size because this can impact the teams' learning processes, and the ability to construct accurate and similar mental models. Team size was measured as the number of team members. We controlled for task experience because it may impact on team learning and team performance. Task experience was measured as the number of participations in previous editions of the competition. We controlled for team familiarity because this can impact the ability to construct similar and accurate mental models. Team familiarity was measured as the percentage of team members that already knew each other before the start of the simulation. We operationalized team composition by transforming the categorical variable into two dummy variables, using the workers as a baseline, since they represented more teams in the competition. We controlled for the group because in this simulation the teams were nested within groups. The different characteristics of each group (the common business environment) may have an impact on performance. 
Chapter 5

\section{RESULTS}

\section{Aggregation}

As the level of analysis in this study was the team, all individual survey responses were aggregated to the team level for further analysis (Costa et al., 2013). To justify aggregation, we computed $r_{\mathrm{wg}()}$ (James et al., 1993), designed for multiple-item scales, and intraclass correlation coefficients (ICCs) (Bliese, 2000). All the mean values of $r_{\mathrm{wg}(\mathrm{j})}$ were in accordance with the required criteria $\left(r_{\mathrm{wg}(\mathrm{j})}>.70\right)$, as well as the values of ICCs: Team learning $\left(r_{\mathrm{wg}())}=0.83 ; \operatorname{ICC}(1)=0.19 ; \operatorname{ICC}(2)=0.52 ; F_{(66,246)}=2.09, p=.00\right)$; team adaptation $\left(r_{\mathrm{wg}(j)}=\right.$ $\left.0.83 ; \operatorname{ICC}(1)=0.15 ; \operatorname{ICC}(2)=0.46 ; F_{(67,241)}=1.85, p=.00\right)$.

\section{Hypotheses testing}

Table 5.1. provides the means, standard deviations, and the correlations for all study variables at the team level. A significant positive correlation was found between team learning and adaptation $(r=.67, p<.01)$, as well as between team adaptation and performance $(r=.31$, $p<.05)$ A non-significant correlation was found between team learning and team performance $(r=-.14, p=.27)$. We continued with the analysis because an input variable (team learning) could indirectly affect an outcome (team performance), even when there is no association between input and outcome variables, through an intervening variable (team adaptation) (Hayes, Preacher, \& Myers, 2011). 


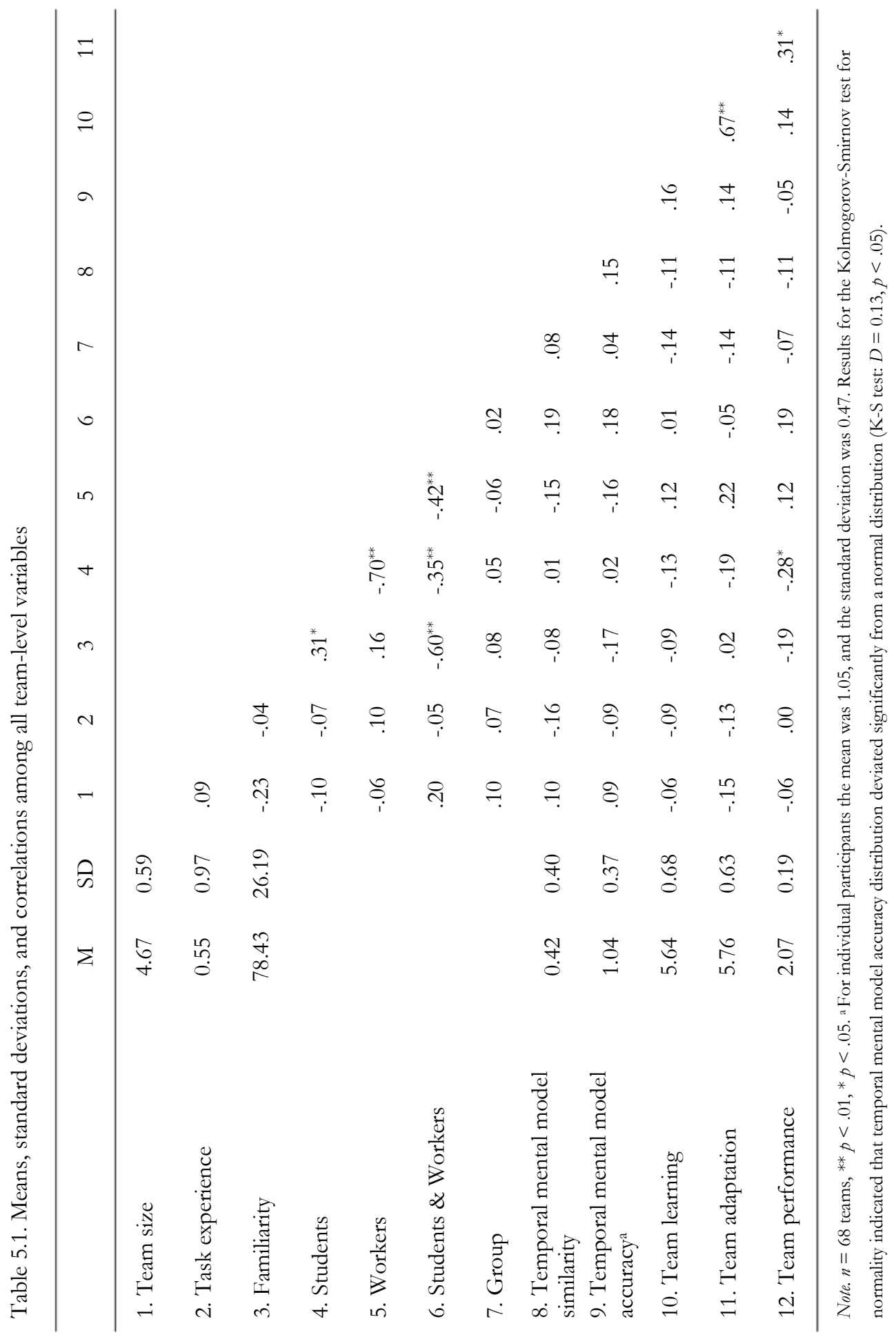


Chapter 5

To analyze the direct effect of mental model similarity (hypothesis 1) and accuracy (hypothesis 2) on team learning, as well as, the moderating effect of mental model accuracy on the relationship between mental model similarity and team learning (hypothesis 3) we performed a step-wise hierarchical multiple regression. Mental model similarity and accuracy were centered, following the procedures suggested by Aiken and West (1991). The block of control variables was entered in the first step and it was not significantly related to team learning $\left(R^{2}=-.05, F_{(6,66)}=0.53, p=.78\right)$. The addition of the main effects for mental model similarity and accuracy at step two did not explain incremental variance in team learning $\left(\Delta R^{2}\right.$ $\left.=.05, F_{(8,66)}=0.79, p=.62\right)$. There was no main effect of mental model similarity $(B=-0.24, t$ $=-1.13, p=.27)$ nor of accuracy $(B=-0.33, t=-1.43, p=.16)$ on team learning. Thus, hypotheses 1 and 2 were not supported. The addition of the interaction between mental model similarity and accuracy at step three explained a significant amount of incremental variance in team learning $\left(\Delta R^{2}=.14, F_{(9,66)}=2.00, p=.05\right)$. The interaction effect of mental model similarity and accuracy on team learning was negative and significant $(B=2.15, t=$ 3.27, $p=.00)$ (see Table 5.2.). 
Table 5.2. Regression results for the interaction effect of temporal mental model similarity and accuracy on team learning

\begin{tabular}{|c|c|c|c|c|c|}
\hline \multirow[b]{2}{*}{ Variables } & \multicolumn{5}{|c|}{ Team learning } \\
\hline & $\mathrm{B}$ & SE B & $F$ & $\mathrm{R}^{2}$ & $\Delta R^{2}$ \\
\hline Step 1: Controls & & & 0.53 & 0.05 & \\
\hline Intercept & $5.74 * * *$ & .18 & & & \\
\hline Team size & -0.07 & .15 & & & \\
\hline Task experience & -0.07 & .09 & & & \\
\hline Team familiarity & -0.00 & .00 & & & \\
\hline Students & -0.17 & .19 & & & \\
\hline Students \& Workers & -0.16 & .28 & & & \\
\hline Group & -0.00 & .00 & & & \\
\hline Step 2: Main effects & & & 0.79 & 0.10 & .05 \\
\hline Intercept & $5.73 * * *$ & 0.17 & & & \\
\hline Team size & -0.06 & .15 & & & \\
\hline Task experience & -0.07 & .09 & & & \\
\hline Team familiarity & -0.00 & 0.00 & & & \\
\hline Students & -0.18 & .19 & & & \\
\hline Students \& Workers & -0.15 & .28 & & & \\
\hline Group & -0.00 & .00 & & & \\
\hline Temporal MM similarity & -0.24 & .22 & & & \\
\hline Temporal MM accuracy & 0.33 & .23 & & & \\
\hline Step 3: Interaction effect & & & $2.00^{*}$ & 0.24 & .14 \\
\hline Intercept & $5.61 * * *$ & .17 & & & \\
\hline Team size & 0.01 & .14 & & & \\
\hline Task experience & -0.09 & .08 & & & \\
\hline Team familiarity & -0.01 & .00 & & & \\
\hline Students & -0.22 & .17 & & & \\
\hline Students \& Workers & -0.37 & .27 & & & \\
\hline Group & -0.00 & .00 & & & \\
\hline Temporal MM similarity & -0.35 & .20 & & & \\
\hline Temporal MM accuracy & $0.53^{*}$ & .22 & & & \\
\hline $\begin{array}{l}\text { Temporal MM similarity } \mathrm{x} \\
\text { Temporal MM accuracy }\end{array}$ & $2.15^{* *}$ & .66 & & & \\
\hline
\end{tabular}

Note. $n=68$ teams. $\mathrm{MM}=$ mental model. ${ }^{*} p<.05 ;{ }^{* *} p<.01 ; * * * p<.001$. 
As the interaction effect was significant, we estimated and graphed the conditional indirect effects to represent high and low levels of mental model accuracy (one standard deviation above the mean and one standard deviation below the mean, respectively; Aiken \& West, 1991; Dawson, 2013). Figure 5.2. shows the interaction effect of mental model similarity and accuracy on team learning. The effect of temporal mental model similarity on team learning was positive and non-significant in a high temporal mental model accuracy condition $(B=0.45 ; t=1.55 ; p=.13)$. By contrast, the effect of temporal mental model similarity on team learning was negative and significant in a low temporal mental model accuracy condition $(B=-1.15 ; t=-3.37 ; p=.00)$. These results showed that with low levels of temporal mental model accuracy the influence of temporal mental model similarity on team learning was negative. Hypothesis 3 was supported.

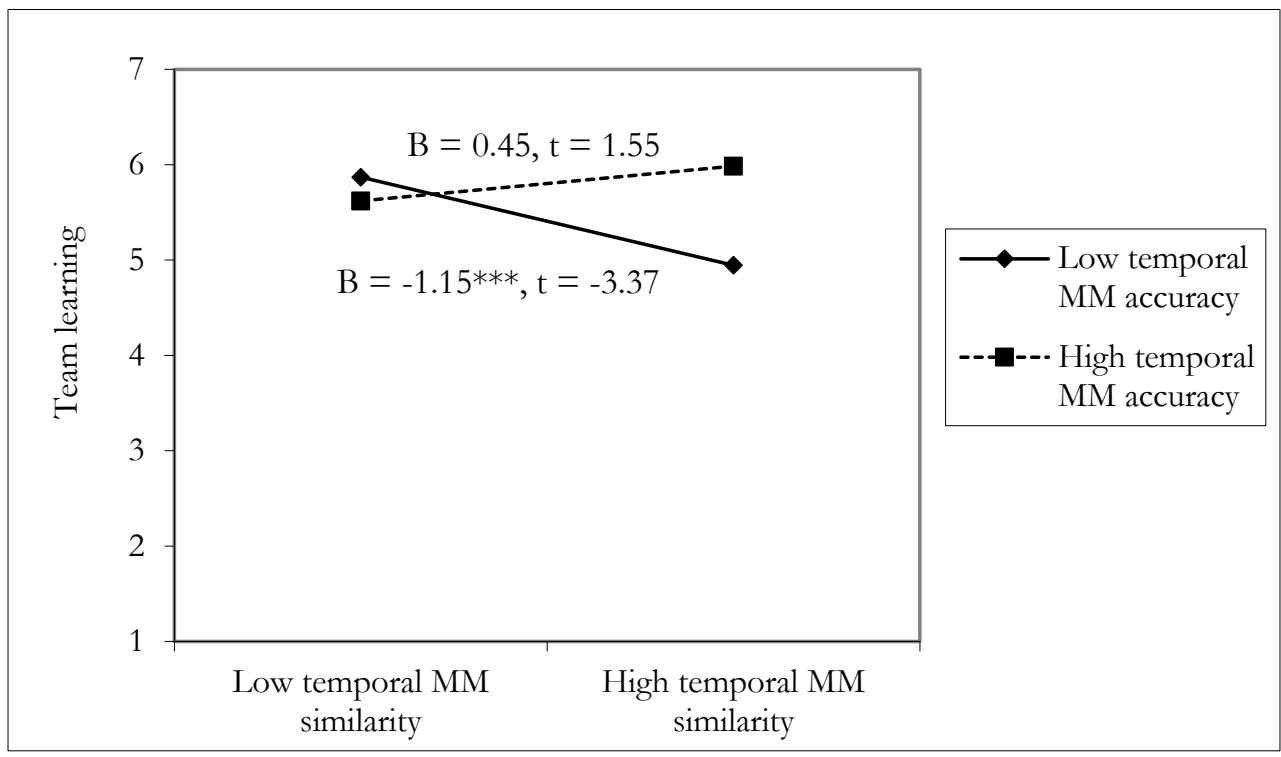

Figure 5.2. Moderation effect of temporal mental model accuracy between temporal mental model similarity and team learning. $\mathrm{MM}=$ Mental model. $* * * p<.001$.

To analyze the mediation effect of team adaptation between team learning and performance we used the statistical software Mplus (Muthén \& Muthén, 2012). We conducted a path analysis with 5000 bootstraps and 95\% confidence interval (CI) (Preacher \& Hayes, 2008). This mediation model was a saturated model, which means that the number of free/estimated parameters equals the number of known values/data points, indicating that the 
model has zero degrees of freedom (Byrne, 2012). For that reason, the overall model fit information was not available. The unstandardized parameter estimate showed that team adaptation positively mediated the relationship of team learning with performance $(.06[\mathrm{CI}$ lower bound $=.03, \mathrm{CI}$ upper bound $=.12], p<.05)$. Hypothesis 4 was supported. Figure 5.3. shows the research model with the direct, mediation, and moderation effects.

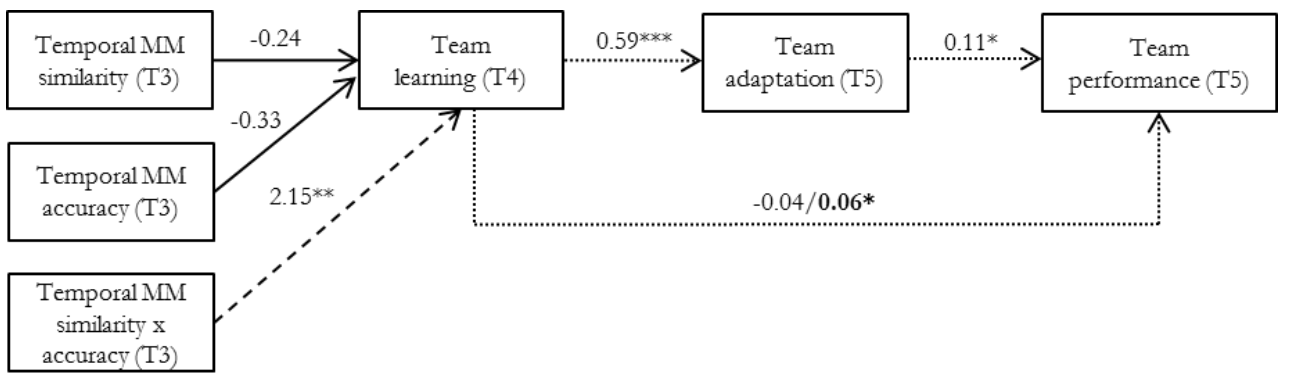

Figure 5.3. Final model representing the results for the hypotheses (unstandardized parameters). In the mediation model, regular numbers represent unstandardized coefficients obtained when modeling mediation. Bold number represents the indirect effect of team learning on performance including team adaptation. $\mathrm{MM}=$ Mental model. $* * * p$ $<.001, * * p<.01, * p<.05$.

\section{DISCUSSION}

Organizational teams are systematically and increasingly pressured by time. Teams need to deal with time pressures and engage in team learning behaviors. We found that when temporal mental model similarity is high and accuracy is low teams engage in less learning behaviors. We also found that team learning is an important booster for team adaptation and performance. Our findings offer important contributions for team research providing insight into how temporal mental models relate to team learning, and into the role team learning plays promoting team adaptation and performance.

Our research advances knowledge about shared mental models and their effects on team learning process. Thus far, researchers have focused on task mental models (a shared understanding about work objectives, and task duties) and on team mental models (a shared understanding about interpersonal interaction, and team members' skills) (Mathieu et al., 2000), neglecting the shared knowledge about the temporal aspects of work. The absence of the temporal dimension of shared mental models hinders researchers to understand how the knowledge about when the tasks should be done may benefit team processes and outcomes 
Chapter 5

(Mohammed et al., 2012). Our research represents a first step on temporal mental models research showing that a similar and inaccurate mental model leads teams to engage less in learning behaviors.

Our findings suggest that there is neither a direct link between temporal mental model similarity and team learning, nor a direct link between temporal mental model accuracy and team learning. Our findings suggest that a similar or an accurate temporal mental model is not enough to promote the engagement of team members in team learning behaviors. Our research may suggest that when team members have a similar or an accurate understanding about the deadlines they need to meet and the time available for each activity, team members trust in the way each other work and accomplish the tasks. Therefore, they do not communicate the things they do, and do not analyze the information together to find solutions for problems or new ways to perform the tasks because they think they are correct. Even when team members make mistakes or have some doubts, team members may not want to share their errors or questions in order to avoid disruptive conflicts (Thompson, 2004).

Nevertheless, our findings suggest that when teams have a high similar and low accurate mental model they are not able to engage in learning behaviors. This means that team members ignore new information or ideas that challenge the ideas they strongly and incorrectly share, and are not willing to discuss the errors they made or the problems that occur over the team lifecycle. Therefore, shared cognition can lead to closed-minds when the knowledge the team members share is incorrect. When team members have a high accurate temporal mental model, they engage in learning behaviors whether they have a low or high similar mental model. As those team members are correct about the most appropriate time to work on the tasks and to discuss the problems, they are able to learn from each other without time pressure and without interfere with the task accomplishment. Therefore, the knowledge about the temporal aspects does not need to be highly shared by all the team members in order to engage in learning behaviors, but the knowledge needs to be correct. Team members do not know whether their mental model is accurate or not. However, when team members have a similar but incorrect temporal mental model they may overestimate the quality of their own cognition. Thus, they ignore ideas and suggestions that challenge the way they think (Thompson, 2004). Team members may think there is no need to learn and improve their strategy because the way they work is correct and sufficient to accomplish the task goals. Team members need to have an accurate temporal mental model in order to engage in team learning behaviors. 
Our findings suggest that when the shared knowledge is incorrect, a highly similar mental model may be disruptive for team functioning. One of the main benefits of teamwork is the diversity of points of view, ideas, and skills of the team members (Kozlowski \& Ilgen, 2006). With an extremely overlap mental model, the diversity benefits may disappear or not emerge in teams. Although team members share a mental model, they should have different perspectives and perceptions on temporal and/or task issues in order to stimulate the discussion about the relevant aspects of team work. If team members do not discuss about and question important aspects of the work, teams may crystalize and they neither update their working methods, nor adapt to (un)expected changes. The effect of an extreme overlapping of mental models on team processes, and even on performance, is a question that deserves attention in future research.

Finally, our research extends knowledge about the effect of team learning on adaptation and performance. Researchers have argued that by engaging in team learning behaviors, team members evaluate past performance episodes, and develop new strategies to overcome previous errors, promoting the team adaptation process, and in turn performance (Burke et al., 2006a; Kozlowski \& Bell, 2008; Rosen et al., 2011). However, there is a lack of empirical work that supports the recent models of team adaptation (Burke et al., 2006a; Rosen et al., 2011). Our research suggests that, indeed, teams that engage in team learning behaviors are able to adapt to changing situations and achieve high levels of performance because those behaviors in which engage result in knowledge that is embedded inside the team and help them to accomplish the team goals (Burke et al., 2006a; Rosen et al., 2011).

\section{Practical implications}

Our study also offers implications for organizational, business, and managing teams. Our findings show that team members should develop and share correct temporal perspectives about the way they work. In the beginning of the team lifecycle is important that team members discuss and share ideas about the best way to allocate the time available for each team activity, plan the work that each team member needs to perform, establish a plan of week activities and agree about time needed to make the weekly decisions. Importantly, whenever possible team members should discuss and share their ideas with someone expert in the task in order to ensure that the knowledge the team members share is correct. This way teams are likely to share an accurate temporal mental model. Furthermore, team members 
Chapter 5

need to be instructed that over the team lifecycle they should discuss the previous task episodes, to explore different perspectives to accomplish the tasks, and communicate and analyze errors that they made in past episodes. If teams know the importance of engaging in these learning behaviors, they are likely to effectively adapt their strategies and procedures, and in turn achieve high levels of performance.

In addition, to avoid a mental model extremely similar and overlapping teams may institute a team member, or even a person outside the team, that plays the role of "devil's advocate". This person would review, retest and question the ideas and assumptions team members have about the way to work, and how and when to work, spotting defects to them. The main goal of the devil's advocate is to falsify the reality and stimulate the debate among team members in order to promote the discussion of different ways to think about the tasks and perform them (Thompson, 2004). The devil's advocate may help teams to engage in learning behaviors, and in turn to appropriately adapt the way they work and to accomplish the tasks with success.

\section{Limitations and directions for future research}

This research was conducted with teams enrolled in a management simulation. Although team members worked together for more than five weeks and participated in a dynamic competition, which required them to deal with complex decision-making and to focus on several indicators to reach a specific objective, the teams were artificial. However, other authors have also used artificially created teams in their research (e.g., Edwards et al., 2006; Mathieu et al., 2000). As with real teams in authentic organizations, teams are highly pressured by time to make decisions, which demand high levels of coordination and interaction. In particular, in the management simulation teams need to make decisions every week, analyze a large amount of information related to various areas of expertise, and coordinate their efforts efficiently to make the best decisions and submit them at a prescheduled moment. The simulation has very rigid weekly deadlines after which teams are not able anymore to submit their decision.

Since all measures were obtained from team members, we were aware there could be possible problems regarding common-method variance (Siemsen et al., 2010). To deal with this, we collected data in different time moments over the five weeks (Brannick et al., 2010; Spector, 2006). However, some authors argue that method variance is "an urban legend" 
(Brannick et al., 2010, p. 408), and that the spurious causes of relationships among variables are related to the mixture of methods and constructs and not related to the methods themselves (Brannick et al., 2010; Lance et al., 2010). In our study, although team learning is significantly correlated to team adaptation, a CFA showed that these two variables are different constructs. Thus, the common method variance threat is minimized in our study.

Future studies should analyze the three dimensions of shared mental models longitudinally. It is important understand whether teams engage in learning behaviors differently depending on the different dimensions of mental models, and whether the development of one mental model dimension influences the development of other dimension. This question is particularly important because a recent paper suggests that the different dimensions of mental models do not develop at the same time and that the development of one dimension may influence the development of another dimension (Maynard \& Gilson, 2014).

A challenge when assessing mental model accuracy is to precisely specify an optimal model to use as referent for assessing the accuracy of participants' mental models. In this study, experts were participants who won previous editions of the simulation. Although we compared the team members' mental model with the experts' mental model, the temporal mental model of the individuals who won a previous edition of the simulation may not be the correct one. They might have won despite having had weak temporal mental models. Future studies may use a wider variety of referent models, including previously successful teams but also subject matter experts, such as the simulation developers or researchers that are familiar with the simulation.

\section{CONCLUSION}

Although shared mental model research has been prospering over the last decades, researchers have neglected the temporal dimension of mental models. By analyzing the joint effect of temporal mental model similarity and accuracy on team learning, our research suggests that when teams have a similar but inaccurate temporal mental model, they engage less in team learning behaviors. Those teams are likely to be closed-minded, which hinder them to accept new ideas or solutions. Further, our findings suggest that team learning fosters team adaptation, and consequently team performance. Our study provides important insights for 
Chapter 5

researchers and practitioners who aim to provide teams with tools for adapting to unexpected situations, and to achieve high levels of performance. 


\title{
ChAPTER $6^{7}$
}

\author{
A TEMPORAL COMMON GROUND FOR LEARNING: \\ THE MODERATING EFFECT OF SHARED MENTAL MODELS \\ ON THE RELATION BETWEEN TEAM LEARNING \\ BEHAVIORS AND PERFORMANCE IMPROVEMENT
}

${ }^{7}$ This chapter is published as:

Santos, C.M., Uitdewilligen, S., \& Passos, A.M. (2015). A temporal common ground for learning: The moderating effect of shared mental models on the relation between team learning behaviours and performance improvement. European Journal of Work and Organizational Psychology, 24, 710-725. DOI: 10.1080/1359432X.2015.1049158. The version in this doctoral thesis is not the copy of record. http://www.tandfonline.com/doi/full/10.1080/1359432X.2015.1049158 
Chapter 6

\begin{abstract}
In this longitudinal study we integrated a team process and a learning curve perspective on team learning and empirically analyzed whether team learning processes lead to performance improvement. In addition, we tested whether this relation is moderated by the similarity of team members' task, team, and temporal mental models. We tested our model on a sample of 67 teams (314 individuals) competing in a management simulation over five consecutive time periods, using random coefficient modelling. Our findings suggest that team learning behaviours do not have a direct effect on the team learning curve, but temporal and task mental models are crucial for the translation of team learning behaviours into performance improvement. We found that when teams have similar task and temporal mental models, engaging in team learning processes is beneficial; whereas, when teams have dissimilar task and temporal mental models, it is detrimental to performance improvement. We did not find a significant effect for the moderating role of team mental model similarity. Our study emphasizes the importance of integrating different perspectives on team learning and provides support for the role of team cognition as a catalyst for team learning.
\end{abstract}


A temporal common ground for learning

\section{INTRODUCTION}

Team learning is essential for organizational teams that need to continuously adapt to changing environments and maintain high levels of performance (Edmondson et al., 2007; Rosen et al., 2011; Savelsbergh et al., 2012). Team learning has been investigated in the literature as a process and as a learning curve (Edmondson et al., 2007). Team learning processes involve behaviours such as asking questions, challenging assumptions, and discussing errors or unexpected outcomes (Edmondson, 1999; Savelsbergh et al., 2009). Team learning curves are reflected in the trajectory of change in team performance over time (e.g., Edmondson et al., 2007; Pisano, Bohmer, \& Edmondson, 2001). Despite conceptual overlap, the process and learning curve perspectives on team learning have been studied in separate research streams. In this study, we combine these two perspectives in order to demonstrate how team learning processes are related to the trajectory of performance over time. Our study aims to demonstrate that team learning processes do not only have an immediate effect on performance but that teams increasingly benefit as they continue to interact and perform over time.

Additionally, scholars have begun to question whether there may be boundary conditions to the positive effects of engaging in team learning processes (e.g. Bunderson \& Sutcliffe, 2003a; Edmondson, 2003; Fiol \& Lyles, 1985). Team learning is a resource intense and socially sensitive process that may detract from core task performance and can result in conflict and tension among the team members (Bunderson \& Sutcliffe, 2003a). We propose that shared mental models (i.e., a shared understanding among team members regarding the relevant aspects of the team task; Klimoski \& Mohammed, 1994) may constitute a critical factor that may determine under what conditions team learning processes may be beneficial. Therefore, we pose that only when teams start with a basic common ground (i.e., share a mental model), they will be able to translate team learning processes into performance improvement.

With this study we aim to contribute to the team learning and team cognition literature in three important ways. First, although a number of authors have posited that more research on how team functioning enfolds over time is needed (e.g., Bliese \& Ployhart, 2002; Cronin et al., 2011; Pitariu \& Ployhart, 2010; Roe, Gockel, \& Meyer, 2012), as yet, evidence is lacking for this longitudinal effect of team learning on team performance improvement. Our longitudinal design allows us to understand this longitudinal effect. Second, by investigating 
Chapter 6

the effect of team learning processes on the increase of team performance over time we integrate the, as yet, separately developed notions of team learning processes and team learning curves. Third, by identifying shared mental models as an important boundary condition for team learning, we shed light on the question of under what conditions team learning processes actually lead to performance improvement. In addressing these gaps, our study aims to provide insights about how teams function and perform over time.

\section{THEORETICAL BACKGROUND AND HYPOTHESES}

\section{The relationship between team learning processes and team performance improvement}

Although there are different conceptualizations of team learning processes (e.g., Wilson, Goodman, \& Cronin, 2007; Schippers, Homan, \& van Knippenberg, 2013) we follow the one by Edmondson (1999). This approach refers to team learning processes as a combination of behaviours, including reflection on processes and outcomes, discussion of important issues, exploration of different perspectives, experimentation with new working methods, analysis and communication of errors, and co-construction of meaning (Edmondson, 1999; Savelsbergh et al., 2009). An important component of team learning processes is team reflexivity - by which team members collectively look back and discuss the team's objectives, strategies, and processes, which helps them to identify potential problems, and to find causes and solutions, and prepare for future action (Schippers et al., 2013; West, 2000). By engaging in learning processes, teams can adapt their strategy and procedures and improve their working methods for subsequent performance episodes (Moreland \& McMinn, 2010; Savelsbergh et al., 2009; Schippers, Den Hartog, Koopman, \& van Knippenberg, 2008). Further, team members can improve their interaction processes, detect and make sense of errors, and prevent the team from making the same mistakes in subsequent task performance episodes (Van Dyck, Frese, Baer, \& Sonnentag, 2005; Schippers, den Hartog, \& Koopman, 2007). Team learning process research has typically employed an input-process-output model, in which team learning behaviours mediate the relationship between relatively stable team or contextual characteristics and team outcomes (e.g., Edmondson, 1999; Wong, 2004).

Team performance improvement is most accurately portrayed by team learning curves (Mathieu \& Rapp, 2009). Team learning curves show the temporal trajectory of team 
performance over time from the beginning of a new product or process. Team learning curves have been studied mainly in operational settings, such as manufacturing or healthcare, where accumulated experience with a task has been consistently linked with improvement in team efficiency (Edmondson et al., 2007). When experience with the task accumulates, teams develop routines and procedures that enable them to reduce the time required to complete their tasks and improve the quality of their performance (e.g., Pisano et al., 2001). Previous research shows that not only the individual experience of the team members matters, but also the experience the members accumulate in working on the task as a team (Reagans, Argote, \& Brooks, 2005). For instance, studies by Pisano and colleagues (2001) and Edmondson, Winslow, Bohmer, and Pisano (2003) showed that cardiac surgery teams gradually improved their efficiency after implementing a new surgical procedure. Moreover, they found that teams varied in the rate at which they learnt to use the new technology.

A number of variables have been identified that impact team learning curves, including task experience and team stability (Edmondson et al., 2003; Pisano et al., 2001; Reagans et al., 2005). However, research on the role of team learning processes on performance improvement is still scarce. Research on individual expertise development suggests that it is not simply the amount of experience with a task but a specific type of dedicated practice that predicts performance increase (Ericsson, Krample, \& Tesch-Römer, 1993). Team learning process is a bottom-up emergent phenomenon that originates at the individual level and emerges through team member interactions, as a team-level construct (Costa et al., 2013; Kozlowski \& Chao, 2012; Kozlowski, Chao, \& Jensen, 2010; Kozlowski \& Klein, 2000). At the individual level, team members acquire knowledge, skills, and performance capabilities that are necessary to accomplish their individual tasks (Kozlowski, Gully, Nason, \& Smith, 1999). Subsequently, team members learn how their task is related to the tasks of the other members. In order to achieve this higher compilation of knowledge, team members have to engage in purposeful interpersonal interactions aimed at gaining an understanding of their teammates' roles and capabilities (Pearsall, Ellis, \& Bell, 2010). Thus, to develop effective interaction processes, team members should engage in processes of communication, reflection, exchange, observation, and collaboration (Kozlowski \& Bell, 2008; Kozlowski \& Chao, 2012). The outcomes of the team learning processes emerge as a pool of declarative as well as procedural knowledge shared among the members of the team and is generally considered to lead to an increase in team performance over time (Edmondson, 1999; Wong, 2004; Savelsbergh et al., 2012). 
To date, various studies have demonstrated a positive relation between team learning processes and team performance (e.g., Guchait \& Hamilton, 2013; Savelsbergh et al., 2009; van der Vegt \& Bunderson, 2005; van Woerkom \& Croon, 2009). These studies suggest that by asking questions, seeking feedback, and reflecting on previous performance episodes, team members test their assumptions, discuss divergent opinions, and thereby achieve high levels of performance (Edmondson, 1999; Guchait \& Hamilton, 2013; Savelsbergh et al., 2009; Schippers, Den Hartog, Koopman, \& Wienk, 2003). However, because in these studies performance was only measured a single time, they cannot provide insight into the effects of team learning processes on the trajectory of team performance. Recently, Schippers and colleagues (2013) conducted a study with business students working on their bachelor thesis over 10 months where they measured team learning and team performance twice. Their findings showed a mediating effect of team learning at Time 2 between the interaction of initial team performance and team reflexivity at Time 2 on final performance, controlling for Time 1 team learning and reflexivity (Schippers et al., 2013). However, in order to assess and predict between-team variations in performance trajectories, such as the slope, at least three data points in time are required (Ployhart \& Vandenberg, 2010). Therefore, the previous studies can only show that team learning is associated with high performance but not that learning behaviours are related to the trajectory of performance over time.

We posit that team learning processes are likely to foster not only immediate team performance but also an increase in team performance over time. Discussing errors about previous tasks helps members to detect faults in their performance routines, improve their strategies, and work more effectively on subsequent tasks (Edmondson, 1999; Savelsbergh et al., 2009). Thus, over time, teams that engage in learning behaviours are likely to develop their collective understanding of the task and optimize the coordination of team members' actions, which lead to improvement in team functioning indicated by a positive performance trajectory (Edmondson et al., 2007).

Hypothesis 1: The extent to which the team members engage in team learning processes is positively related to team performance improvement. 
A temporal common ground for learning

\section{Shared mental models as a catalyst for team learning}

Several studies indicate that the extent to which teams engage in team learning processes is positively related to team performance (Guchait \& Hamilton, 2013; Savelsbergh et al., 2009; Wong, 2004); however, these processes may not always translate into team performance improvement (Bunderson \& Sutcliffe, 2003a; Edmondson, 2003; Fiol \& Lyles, 1985; Kozlowski et al., 2010). First, team learning behaviours are intense and consume time and cognitive resources (Bunderson \& Sutcliffe, 2003a, 2003b). The cognitive resources a team has at its disposal for performing tasks are limited (Barnes et al., 2008; Kanfer \& Ackerman, 1989). When teams allocate their cognitive resources away from their assigned tasks, for instance to engage in team learning behaviours, or to reflect about their work, team members make an additional effort representing an extra cost for their cognitive resources (Bunderson \& Sutcliffe, 2003a). Therefore, when team members exert efforts on team learning processes, it is crucial that they spend their resources efficiently; otherwise team performance is likely to suffer. Further, teams that engage in team learning behaviours may abandon adequate solutions and choose untested approaches without gaining benefits (Barnes et al., 2008; MacMillan, Entin, \& Serfaty, 2004). This may create too much variation in teams' alternatives that cannot be effectively assimilated by team members and may consequently harm team performance (Bunderson \& Sutcliffe, 2003a, 2003b). Finally, engaging in team learning behaviours can be challenging for team members and may involve interpersonal risk taking (Edmondson, 2003). Particularly, when team members have diverging perceptions on central aspects of the task, they may not be willing to ask questions, seek information or admit mistakes (Edmondson, 2003). Thus, when team members do not have similar mental models, team learning behaviours may be ineffective and time consuming, which hinders team performance improvement.

Several scholars have noted that team cognition, and in particular shared mental models, may have a critical function in the relation between team learning processes and team performance (e.g., Decuyper, Dochy, \& Van den Bossche, 2010; Tindale et al., 1996, 2008). Shared mental models refer to a common understanding by the team members regarding task, team, and temporal aspects of their work (Klimoski \& Mohammed, 1994; Mohammed et al., 2010). Shared task mental models refer to a similar understanding among the team members about work objectives, team resources, and task duties (Mathieu et al., 2000; Mohammed et al., 2010). Shared team mental models refer to a shared understanding regarding interpersonal 
Chapter 6

interaction, and team members' skills (Mathieu et al., 2000; Mohammed et al., 2010). Shared temporal mental models refer to a similar understanding about the sequencing of the separate elements of the team task, the pacing with which activities should take place, and the deadlines for task accomplishment (Standifer \& Bluedorn, 2006).

A wide variety of studies indicates that when team members have similar mental models, they are likely to achieve high performance levels because they are able to accomplish the tasks efficiently without the need for explicit coordination and communication (DeChurch \& Mesmer-Magnus, 2010). What is more, when team members have a similar mental model, they will interpret changes in the task environment in a compatible way (Cannon-Bowers et al., 1993). This enables them to anticipate the needs and actions of other members while dealing with these changes (DeChurch \& Mesmer-Magnus, 2010; Gevers et al., 2006; Rico et al., 2008). Further, when teams have similar mental models, they are on the same page regarding the functioning of the team, the team's strategy, and when deadlines have to be met (Mohammed et al., 2010; Mohammed \& Nadkarni, 2014). This is likely to facilitate the synchronization of learning processes among the team members, enable efficient communication, and ensure that the learning processes are aligned with collectively agreed upon goals (Gevers et al., 2006; Kozlowski \& Chao, 2012). In contrast, when team members do not have similar mental models, team learning may lead to misunderstandings, process loss, and frustration among the team members. Therefore, we expect that when team members have a similar mental model, this constitutes a fertile breeding ground for team learning to occur (Tindale et al., 2008). Sharing a mental model promotes effective team learning processes and the positive trajectory of team performance because "the more knowledge that team members share in common, the better able they are to apply it to solving the problem or making the decision" (Kozlowski \& Chao, 2012, p. 343). Similar mental models ensure that team members can quickly make sense of suggestions made by their teammates, that they discuss information, strategies, and problems that are aligned with the team goals, and that they provide each other with appropriate feedback at the right moment in time (Tindale et al., 2008).

\section{Shared task, team, and temporal mental models and team learning}

We expect that all three dimensions of mental models moderate the relationship between team learning processes and performance improvement. When teams share a task 
mental model they have a similar understanding about how the task should be accomplished in terms of procedures and practices, as well as about the resources needed to accomplish the task (Cannon-Bowers et al., 1993). Previous research shows that a shared understanding of the information elaboration requirements of the task is related to the quality of information sharing among the team members (van Ginkel, Tindale, \& van Knippenberg, 2009). Team members that have a similar task mental model are more likely to communicate information in a way that is understood by the recipients (Fussell \& Krauss, 1989; Krauss \& Fussell, 1991). For instance, when a team member suggests a novel idea, the other members can directly make sense of this as they can place it into their own mental schema. In contrast, when team members have strongly divergent task mental models, they are more likely to misunderstand each other or require extensive communication in order to make sense of each other's ideas and suggestions (Cronin \& Weingart, 2007). In addition, a shared task mental model ensures that team members agree on what issues are central to task accomplishment. This ensures that team learning processes will be directed at issues that are considered important by all team members. Thus, shared task mental models facilitate team learning behaviours by ensuring that feedback and suggestions provided by team members are aligned with the team goals and are easily communicated and understood by the other members.

Hypothesis 2a: The relationship between team learning processes and team performance improvement is moderated by task mental model similarity in such a way that when team members have a similar task mental model, the relationship will be more positive than when they do not have a similar mental model.

When team members have a shared team mental model, they have a similar understanding about the team interaction, their responsibilities, the relation between their roles, and the knowledge, skills and abilities of each team member (Cannon-Bowers et al., 1993). A shared understanding among the team members on how to interact with each other is likely to facilitate a variety of team processes. Previous studies have found a positive relation between shared team mental models and team processes, including communication and coordination (e.g., Mathieu et al., 2000). Given that team learning processes are in essence communication behaviours (Savelsbergh et al., 2009), they are likely to benefit from shared team mental models as well. Moreover, a clear understanding by team members of the distribution of roles and responsibilities in the team can increase the efficiency of teams' learning efforts as each member can focus on the tasks he or she is most experienced with. In 
Chapter 6

addition, when members' are aware of each other's strengths and weaknesses, this will enable them to understand which members may need help or feedback and which members may help the team to understand and overcome previous errors or unexpected outcomes. Thus, a shared team mental model provides team members with an agreed upon structure for the interaction and discussion processes that are central to team learning and provides an efficient task distribution for the learning process (Edmondson, 2003; Savelsbergh et al., 2009).

Hypothesis 2b: The relationship between team learning processes and team performance improvement is moderated by team mental model similarity in such a way that when team members have a similar mental model the relationship will be more positive than when they do not have a similar mental model.

Finally, when team members share a temporal mental model, members have a shared understanding about the sequencing of the teams' tasks, deadlines for task accomplishment, and the pacing at which activities should take place (Gevers et al., 2006; Standifer \& Bluedorn, 2006). A shared temporal mental model can enable a team to optimize the timing and synchronization of team learning processes. Suggestions and feedback that are relevant but not voiced at appropriate times are less likely to be picked up by other team members (Kulik \& Kulik, 1988). Moreover, given that sustainable team functioning depends on a careful balancing of exploitation activities_engaging in immediate task performance-and exploration activities - collectively improving task processes for future task activities - it is crucial that team members time their team learning behaviours in such a way that they do not interfere with core task performance (Bunderson \& Sutcliffe, 2003a; March, 1991; Mohammed \& Nadkarni, 2014). Therefore, when team members are on the same page regarding when deadlines have to be met, when tasks have to be executed, and when there is time for reflection, learning processes will be synchronized among the team members as well as with the ongoing requirements of the execution of the team tasks. The research model is represented in Figure 6.1.

Hypothesis 2c: The relationship between team learning processes and team performance improvement is moderated by temporal mental model similarity in such a way that when team members have a similar temporal mental model the relationship will be more positive than when they do not have a similar temporal mental model. 


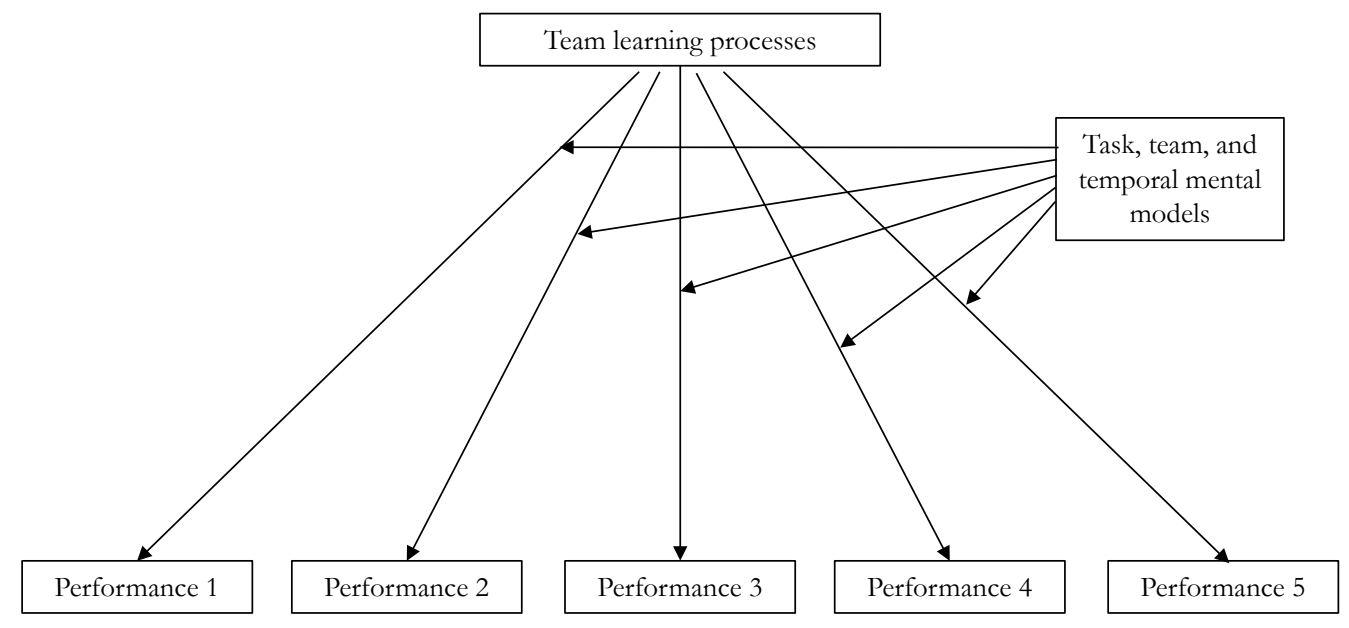

Figure 6.1. Hypothesized research model.

\section{METHOD}

\section{Sample}

The sample of the study consisted of 67 teams (314 individuals) enrolled in a management simulation over a 5 -week period. The teams were composed of company managers (45.6\%), university students $(36.8 \%)$, or both $(17.6 \%)$. The teams consisted of three to five persons with an average team size of 4.67 persons $(S D=0.58)$. The majority of teams contained five persons $(73.1 \%)$. The average age was 30.3 years $(S D=7.35)$ and $73.5 \%$ of the participants were male.

\section{Simulation}

Data were collected from the participants of a national management and strategy simulation - Global Management Challenge ${ }^{\circledR}$. This simulation has been used by others researchers (e.g., Costa et al., 2014; Marques-Quinteiro et al., 2014; Santos \& Passos, 2013). During the simulation each team runs a fictitious company with the objective of getting the highest company share price on the simulated stock exchange. The simulation lasts 5 weeks. Teams manage the company by making decisions each week about marketing, production, personnel, purchasing, and finance. Each team has a leader chosen by the team members at 
Chapter 6

the moment they enrolled for the simulation. For each week, the business simulation programmme analyses and compares the decisions made by the competing teams, and calculates the share price of each enterprise and the ranking of teams. Then, the programme produces a management report for each team, showing detailed results in financial terms.

\section{Procedure}

Online questionnaires were sent to the team members by e-mail, two days after the beginning of the weekly task. A reminder was sent to the participants one day before the deadline to submit the weekly decision. The questionnaires were available until the moment in which teams had to submit their weekly decision. Participants individually answered the questionnaires before receiving the management report with the feedback about their decisions. This longitudinal procedure reinforces causality inferences (Mathieu \& Taylor, 2006) and reduces common-method variance (Brannick et al., 2010; Spector, 2006).

\section{Measures}

Shared mental models. To operationalize shared mental models, we used the procedure that is used most generally and considered the most valid in mental model research (DeChurch \& Mesmer-Magnus, 2010; Resick et al., 2010b). We asked participants to evaluate the relatedness of sentences that describe team, task and temporal procedures that are relevant for the team task, on a 7 -point scale $(1=$ the sentences are not related and $7=$ the sentences are extremely related $)$.

To operationalize the team mental models, we adapted four items of the mental model measure developed by Lim and Klein (2006). For the task and temporal mental models, we created - based on a detailed task analysis and with the help of the company developers of the simulation - four sentences that fit the specific task context. For the task mental model, we derived four sentences regarding the task aspects of the simulation, and for temporal mental models we derived four sentences regarding the temporal aspects of the simulation (Mathieu \& Rapp, 2009; Uitdewilligen et al., 2013). Team members were asked to make paired comparisons among the four sentences of each dimension resulting in six comparisons for each dimension. A list of pairs of sentences is provided in the Appendix D.

To assess the similarity of the mental models among the team members, we used UCINET (Borgatti et al., 1992), following the procedure developed by Mathieu and colleagues 
(2000). This network-analysis programme provides a similarity measure based on Pearson's correlations ranging from -1 (completely dissimilar) to 1 (completely similar). We entered the relatedness scores of the team members into matrices for each team. Then we used UCINET to calculate the team similarity among the matrices of the team members.

Team learning processes. Team learning processes were measured using the scale of Savelsbergh and colleagues (2009) which covers eight dimensions: co-construction of meaning, exploring different perspectives, error analysis, error communication, reflection on processes, reflection on outcomes, feedback seeking behaviour, and experimenting. We opted for this measure because it provides a comprehensive overall measure of team learning behaviours that fits well with the conceptualization of team learning we adopted from Edmondson (1999). The scale validation study by Savelsbergh and colleagues (2009) indicates that team learning can be operationalized as a second-order construct composed of the eight behavioural dimensions. As we aim to analyse the influence of team learning behaviours as a whole, we operationalized team learning as the average score of all items. Participants rated the extent to which they agreed with each sentence (e.g., "We encourage each other to look at our work from different perspectives") on a 7 -point scale $(1=$ totally disagree; $7=$ totally agree). Team learning was measured in the fourth week of the simulation. The Cronbach's alpha was .98. A second-order confirmatory factor analysis for the eight dimensions of team learning processes presents an acceptable, although not perfect, model fit: $\chi^{2}{ }_{(292)}=1212.72, p$ $=.00 ; \mathrm{CFI}=.88 ; \mathrm{TLI}=.87 ; \mathrm{SRMR}=.05$.

Team performance. Team performance was operationalized as the share price in each week of the simulation. Share price was given in Euros and was automatically calculated by the simulation. Share price is a complex outcome measure that combines the decisions on the distinct topics on which team members need to make decisions (e.g., production, finance, and marketing), and thereby captures the combined effort of the team as a whole.

Control variables. We included team size, task experience, and shared mental model accuracy as control variables in our analyses (Humphrey et al., 2009). Team size can impact the team learning processes and the team's ability to develop mental models. We also controlled for task experience (participation in previous editions of the simulation) as this may impact mental models, team learning processes, and outcomes. In addition, we controlled for 
Chapter 6

shared mental models accuracy (the extent to which the team members' mental models are similar to those of experts in the respective area; Edwards et al., 2006) as this may correlate with mental models similarity, and impact team learning processes and outcomes. Mental model accuracy was assessed by comparing the mental models of the team members to an expert model based on ratings of 12 participants who had won previous editions of the competition (Webber et al., 2000).

\section{Aggregation}

As the level of analysis in this study was the team level, the individual responses to the team learning questionnaire were aggregated to the team level for further analysis (Costa et al., 2013). We computed $R_{\mathrm{wg}()}$ indicators (James et al., 1993), designed for multiple-item scales, and intraclass correlation coefficients (ICC) (Bliese, 2000) to justify aggregation. All the values were in accordance with the required criteria $\left(\mathrm{R}_{\mathrm{wg}(\mathrm{f})}=0.83 ; \operatorname{ICC}(1)=0.19 ; \operatorname{ICC}(2)=0.52\right.$; $\left.F_{(66,246)}=2.09, p=.00\right)$.

\section{Data Analysis}

To analyse our longitudinal data and test our hypotheses we used RCM following the guidelines by Bliese and Ployhart (2002). RCM accounts for nonindependence of observations and for heteroscedasticity (inconsistency in the variance over time). In addition, it provides tests for intrateam and interteam change and enables the analyses of team performance trajectories (Bliese \& Ployhart, 2002). We estimated all our models in the statistical software R (version 3.1.1), an open source statistical software well suited for RCM (Culpepper \& Aguinis, 2011). We estimated the growth models by means of the Nonlinear and Linear Mixed Effects package written by Pinheiro and Bates (2000). We coded time as 0, 1, 2, 3, and 4 to represent weeks 1, 2, 3, 4, and 5, respectively. In this way, we were able to interpret the intercept of our performance growth model as the performance score on the first trial (Bliese \& Ployhart, 2002). We grand-mean-centred our dependent and independent variables to ease interpretation and enable cross-model comparison (Singer \& Willett, 2003). 
A temporal common ground for learning

\section{RESULTS}

Table 6.1. provides the means, standard deviations, and the correlations for all study variables at the team level. The results did not show significant correlations between performance and mental model similarity, nor between team learning processes and performance. Regarding the control variables, team size was negatively correlated with task and team mental model similarity $(r=-.27, p<.05 ; r=-.38, p<.01$, respectively). To test our hypotheses, we followed a number of steps divided into two levels. In level 1, we established the fixed functions for time and in level 2, we added predictors of intercept and slope variability to test our hypothesized relationships (Bliese \& Ployhart, 2002). 


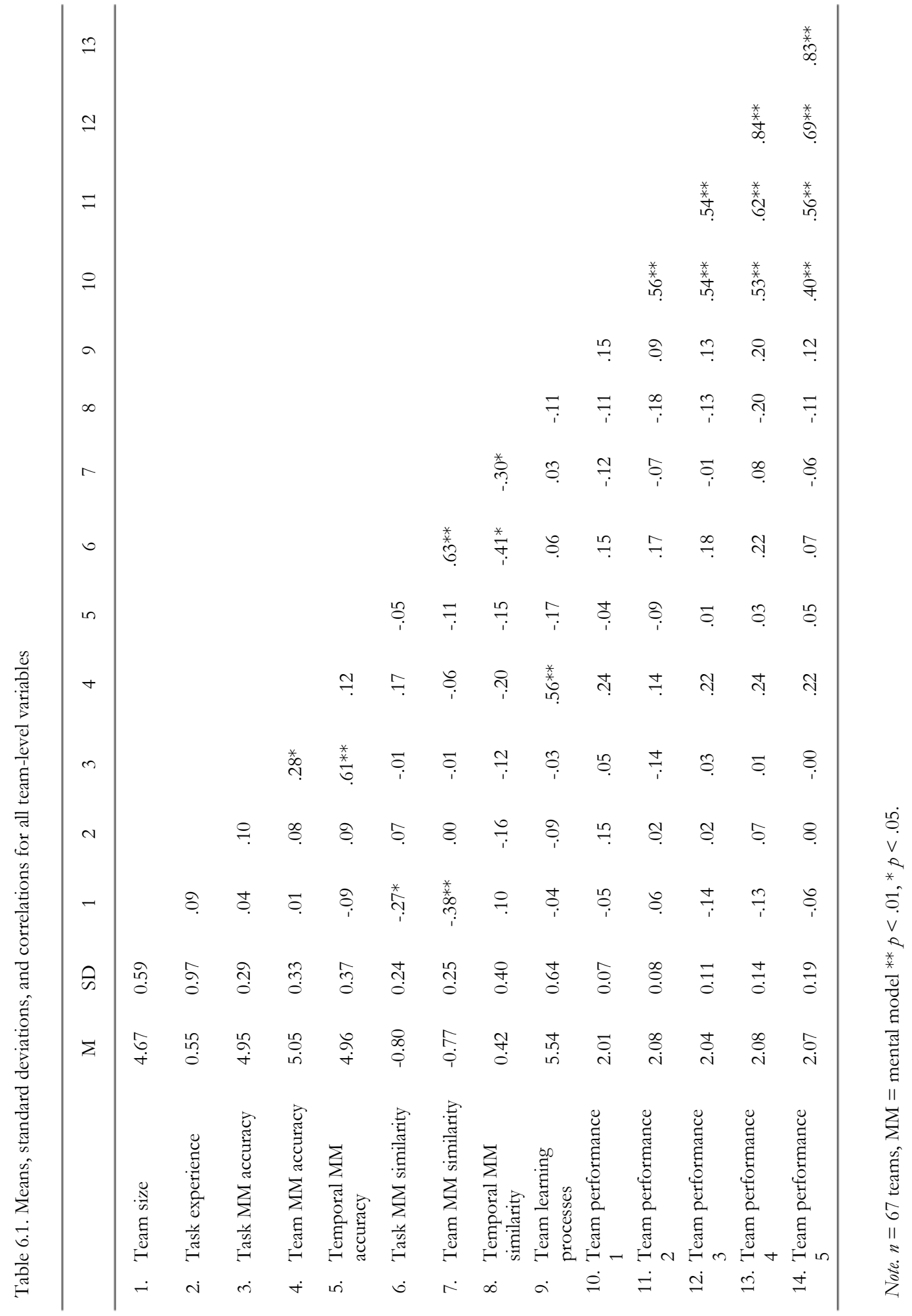


We examined the ICC(1) for the dependent variable, team performance, to determine the total amount of variance that is attributable to between-team (over time) versus withinteam differences (Bliese, 2000). The ICC(1) value for team performance was .27, which means that $27 \%$ of the variance was attributable to between-team differences and $73 \%$ of the variance was attributable to within-team differences over time. As there is sufficient withinteam variance, growth models are an appropriate technique for analysing these data (Bliese, 2000).

In order to test the average trajectory of team performance of all teams over time, we first determined the fixed relation between the variable time and team performance. The results indicate that the estimate of the linear function for time was positive and statistically significant $(t=2.73, p<.01)$, suggesting that on average the teams showed an increase in performance scores. In addition to a linear effect, we also tested a quadratic effect of time on team performance. However, the quadratic function of time was not significant $(t=-1.66, p=$ $.10)$.

Next, we analysed whether there is significant variance between the teams in the intercept and slope of performance over time. To determine variability in the growth parameters, we first added a random intercept term to our model to test between-team differences in the initial levels of team performance (see Table 6.2.). This step aims to examine whether a random intercept model (i.e., teams significantly differ in their initial team performance level) fits our data better than a fixed intercept model. In order to establish the optimal model and most parsimonious model, we used chi-square difference (i.e., -2 loglikelihood ratios (-2LL)) to compare the more complex model with the baseline model (Bliese \& Ployhart, 2002). The comparison of the random-intercept model $(-2 \mathrm{LL}=274.34$ ) with the baseline model $(-2 \mathrm{LL}=211.70)$ significantly improved the model fit $(\Delta 2 \mathrm{LL}=62.64$, $p<.001)$. This means that the model that allows teams to differ in their initial team performance fitted the data better than the model with a fixed intercept. 
Chapter 6

Table 6.2. Results of fixed function for time (Model 1), and of fitting random coefficient models to team performance (Models 2 and 3).

\begin{tabular}{|c|c|c|c|c|c|c|c|c|c|}
\hline \multirow{2}{*}{$\begin{array}{l}\text { Parameter } \\
\text { Fixed } \\
\text { effects }\end{array}$} & \multicolumn{3}{|c|}{$\begin{array}{l}\text { Model 1: Linear } \\
\text { function for time }\end{array}$} & \multicolumn{3}{|c|}{$\begin{array}{c}\text { Model 2: Random } \\
\text { Intercept }\end{array}$} & \multicolumn{3}{|c|}{$\begin{array}{l}\text { Model 3: Random } \\
\text { intercept and slopes }\end{array}$} \\
\hline & Estimate & SE & $t$ & Estimate & SE & $t$ & Estimate & SE & $t$ \\
\hline Intercept & $2.03^{* * *}$ & 0.01 & 170.43 & $2.03 * * *$ & 0.01 & 146.12 & $2.03 * * *$ & 0.01 & 262.04 \\
\hline Time & $0.01 * *$ & 0.00 & 2.73 & $0.01 * * *$ & 0.00 & 3.97 & $0.01 * *$ & 0.01 & 2.58 \\
\hline \multicolumn{10}{|l|}{ Goodness of fit } \\
\hline $\begin{array}{l}-2 \text { log- } \\
\text { likelihood }\end{array}$ & 211.70 & & & 274.34 & & & 336.06 & & \\
\hline AIC & -417.41 & & & -540.68 & & & -660.12 & & \\
\hline BIC & -405.98 & & & -525.45 & & & -637.27 & & \\
\hline
\end{tabular}

Note. $n=67$ teams, ${ }^{* * *} p<.001, * * p<.01$.

Second, we determined whether there was significant variability among teams in the rate of change in team performance (i.e., slope variation). The random-slope model (allowing for difference in the slope among teams) significantly improved upon the random-intercept model $(\Delta 2 \mathrm{LL}=61.72, p<.001)$. This suggests that the best model accounts for difference in team performance between teams at the beginning of the simulation, as well as for difference in the rate of change across teams.

In addition, we tested for autocorrelation and heteroscedasticity. The results revealed that the models in which we controlled for autocorrelation $(\Delta 2 \mathrm{LL}=0.02, p=.83$ ) and heteroscedasticity $(\Delta 2 \mathrm{LL}=1.77, p=.17)$ did not improve model fit. So, we did not control for autocorrelation and heteroscedasticity in the further analyses.

\section{Level 2 analyses: Predictors of team performance trajectories}

In the first part of the RCM analyses, we determined the relationship between team performance and time. In this second part of the RCM analyses, we estimated a model that included team learning processes and the three dimensions of mental model similarity to 
predict variance in the trajectory parameters. All level-2 models include control variables for team size, task experience, and mental model accuracy.

Hypothesis 1 states that team learning processes are positively related to performance improvement. To test this hypothesis, we added team learning processes to the longitudinal model. The team learning processes were not significantly related to initial team performance $y=-0.00, t=-0.13, p=.90)$ nor was the interaction between time and team learning processes $(y=0.01, t=0.81, p=.42$ ) (see Table 6.3.). Thus, team learning processes did not have a positive main effect on initial team performance neither on performance improvement over time. Hypothesis 1 is not supported.

Table 6.3. Results of team learning processes predicting team performance (Model 4).

\begin{tabular}{lccc}
\hline \multicolumn{1}{c}{ Predictor } & \multicolumn{3}{c}{ Model 4 } \\
\hline Fixed effects & Estimate & SE & $t$ \\
\hline Intercept & $2.05^{* * *}$ & 0.06 & 31.81 \\
Time & $0.01^{*}$ & 0.01 & 2.45 \\
Team size & -0.01 & 0.01 & -0.42 \\
Task experience & 0.01 & 0.01 & 0.88 \\
Task MM accuracy & -0.00 & 0.04 & -0.12 \\
Team MM accuracy & 0.04 & 0.03 & 1.25 \\
Temporal MM accuracy & -0.02 & 0.03 & -0.64 \\
Team learning processes & -0.00 & 0.02 & -0.13 \\
Time x Team learning processes & 0.01 & 0.01 & 0.81 \\
& & & \\
Goodness of fit & & & \\
-2 log-likelihood & 310.77 & & \\
AIC & -595.53 & & \\
BIC & -546.51 & & \\
\hline
\end{tabular}

Note. $n=67$ teams, $\mathrm{MM}=$ mental model, ${ }^{* * *} p<.001, * p<.05$. 
Chapter 6

Hypothesis 2 predicts that the relationship between team learning processes and team learning outcomes over time is moderated by mental models similarity. To test this hypothesis, the interaction terms of team learning processes with the three types of mental models were added to the model. For the task dimension of mental models, the results showed that the interaction term involving time, team learning processes and task mental model similarity was significant $(y=0.13, t=2.10, p=.04$ ) (see Table 6.4.). This interaction is depicted in Figure 6.2. For teams that have a similar task mental model, the extent to which teams engage in team learning processes is related to their initial performance level, whereas for teams that do not have a similar task mental model, this is not the case. Further, the performance level increases over time when teams have a similar task mental model and engage in many learning behaviours. When teams engage in many learning processes and have a dissimilar task mental model, their performance slightly decreases over time. Thus, when teams have a common ground, team learning is beneficial for performance, but when teams do not have a common ground, team learning is detrimental to performance. These results provide support for hypothesis $2 \mathrm{a}$. 
Table 6.4. Results of main and interaction effects of task, team, and temporal mental model similarity predicting team performance.

\begin{tabular}{|c|c|c|c|}
\hline \multirow{2}{*}{$\begin{array}{ll}\text { Predictors } \\
\text { Fixed effects }\end{array}$} & \multicolumn{3}{|c|}{ Model 5} \\
\hline & Estimate & SE & $t$ \\
\hline Intercept & $2.08^{* * *}$ & 0.07 & 30.52 \\
\hline Time & $0.01 * *$ & 0.01 & 2.77 \\
\hline Team size & -0.01 & 0.01 & -0.85 \\
\hline Task experience & 0.01 & 0.01 & 0.86 \\
\hline Task MM accuracy & 0.01 & 0.04 & 0.24 \\
\hline Team MM accuracy & 0.02 & 0.03 & 0.61 \\
\hline Temporal MM accuracy & -0.04 & 0.03 & -1.26 \\
\hline Team learning processes & -0.00 & 0.02 & 0.04 \\
\hline Time $\mathrm{x}$ Team learning processes & -0.00 & 0.01 & -0.09 \\
\hline Task MM similarity & $0.12^{*}$ & 0.05 & 2.20 \\
\hline Team MM similarity & $-0.12^{*}$ & 0.06 & -2.00 \\
\hline Temporal MM similarity & -0.02 & 0.02 & -0.93 \\
\hline Time x Task MM similarity & -0.00 & 0.03 & -0.13 \\
\hline Team learning processes $\mathrm{x}$ Task MM similarity & 0.07 & 0.10 & 0.74 \\
\hline Time x Team learning processes x Task MM similarity & $0.13^{*}$ & 0.06 & 2.10 \\
\hline Time x Team MM similarity & 0.01 & 0.03 & 0.27 \\
\hline Team learning processes $\mathrm{x}$ Team MM similarity & 0.07 & 0.10 & 0.72 \\
\hline Time $\mathrm{x}$ Team learning processes $\mathrm{x}$ Team MM similarity & -0.04 & 0.06 & -0.60 \\
\hline Time x Temporal MM similarity & -0.01 & 0.01 & -0.52 \\
\hline Team learning processes $\mathrm{x}$ Temporal MM similarity & 0.00 & 0.03 & 0.02 \\
\hline $\begin{array}{l}\text { Time X Team learning processes x Temporal MM } \\
\text { similarity }\end{array}$ & $0.07 * * *$ & 0.02 & 3.16 \\
\hline \multicolumn{4}{|l|}{ Goodness of Fit } \\
\hline$-2 \log$-likelihood & 292.49 & & \\
\hline AIC & -534.98 & & \\
\hline $\mathrm{BIC}$ & -441.64 & & \\
\hline
\end{tabular}

Note. $n=67$ teams, $\mathrm{MM}=$ mental model, ${ }^{* * *} p<.001, * * p<.01, * p<.05$. 

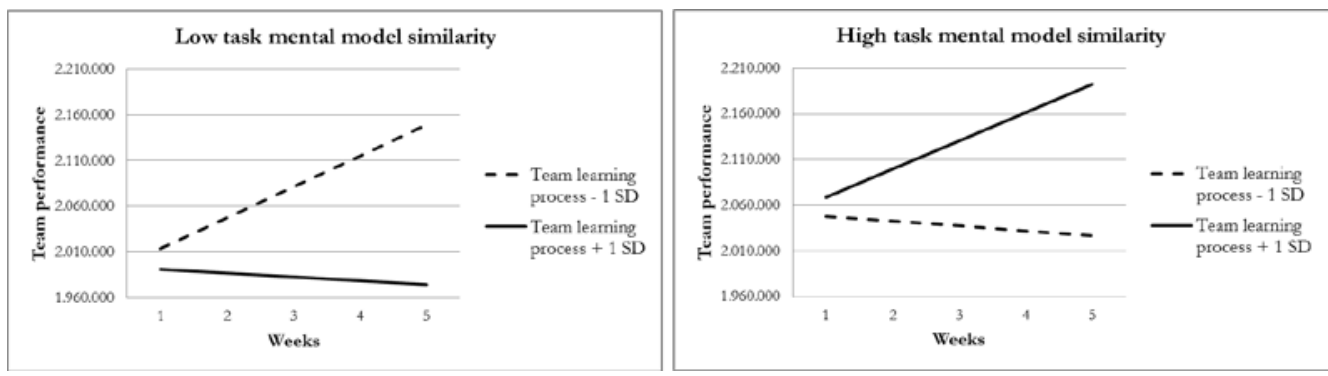

Figure 6.2. The interaction effect between team learning processes and task mental model similarity on team performance.

For the team dimension of mental models, the results showed that the interaction term involving time, team learning processes and team mental model similarity was not significant $(y=-0.04, t=-60, p=.55$ ) (see Table 6.4.). These results do not support hypothesis $2 \mathrm{~b}$.

For the temporal dimension of mental models, the result showed that the interaction term involving time, team learning processes and temporal mental model similarity was significant $(y=0.07, t=3.16, p=.00)$. There is a positive linear trend for the interaction effect between the three variables on team performance (see Table 6.4.). Figure 6.3. shows the interaction between time, low and high levels of temporal mental model similarity (respectively), and team performance. When teams have a similar temporal mental model and engage in many team learning processes, team performance increases over time. When teams have a similar temporal mental model and engage in few learning behaviours, team performance decreases over time. However, when teams have a dissimilar temporal mental model and engage in few learning processes, the performance level increases over time. When teams have a dissimilar temporal mental model and engage in many learning processes their performance is stable over time. Thus, when teams have a temporal common ground, team learning is beneficial to performance, but when teams do not have a temporal common ground, team learning is detrimental to performance. These results provide support for hypothesis $2 \mathrm{c}$. 

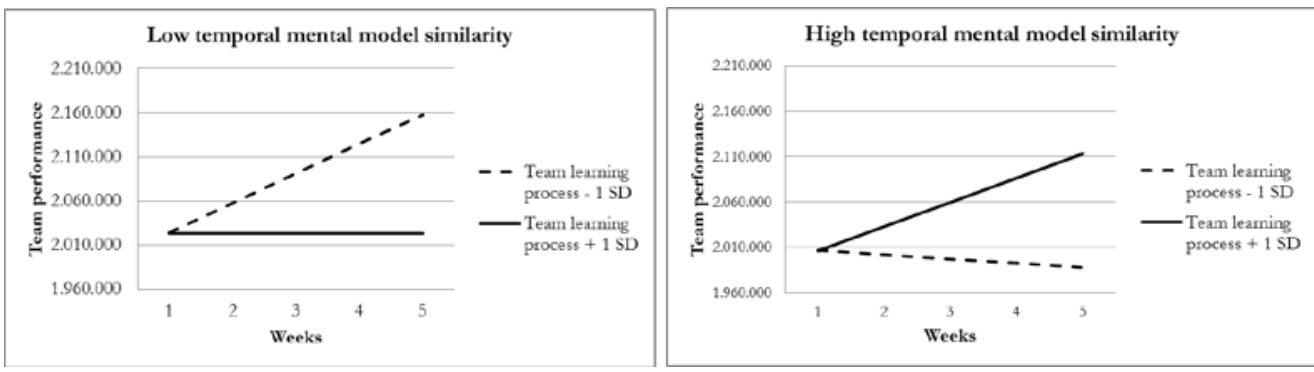

Figure 6.3. The interaction effect between team learning processes and temporal mental model similarity on team performance.

\section{DISCUSSION}

When does engaging in team learning processes benefit team performance improvement? We found that when task and temporal mental model similarity were high, the extent to which teams engaged in collective learning processes was positively related to team performance improvement. However, when task and temporal mental model similarity were low, this pattern reversed, so that learning processes negatively contributed to performance improvement. We did not find a significant effect for the moderating role of team mental model similarity. We tested our hypotheses in a longitudinal study with five measurement points for team performance and the model variables measured at different moments in time. Our findings offer meaningful contributions to the team learning as well as the team cognition literature, and emphasize the importance of longitudinal studies for providing insight into how teams learn and develop over time.

Our primary contribution lies in identifying shared cognition as an important boundary condition for the effect of team learning processes on the development of team performance over time. Thereby, we tie into the debate on whether engaging in collective learning process is always beneficial for team performance improvement. A number of studies have shown that team learning is beneficial for team performance (e.g., Edmondson, 1999; Guchait \& Hamilton, 2013; Savelsbergh et al., 2009). However, recently, Bunderson and Sutcliffe (2003a) questioned whether team learning behaviours are always beneficial for team performance. In a related vein, Moreland and McMinn (2010) wondered what conditions have to be met for team reflexivity to have identifiable effects on performance. We found that team performance only increases over time when teams engage in team learning processes and have 
Chapter 6

similar task and temporal mental models. In order to effectively discuss ideas, analyse errors, and process feedback team members need to be on the same page regarding the team strategy, deadlines for task accomplishment, and the pacing at which activities take place (CannonBowers et al., 1993; Gevers et al., 2006; Standifer \& Bluedorn, 2006). Lack of shared understanding may lead members to experience task conflict - intragroup disputes related to the content of the tasks, such as differences regarding ideas or opinions (Jehn, 1995), and temporal conflict - intragroup disagreements about time, the duration of a task, and the length of time the team should spend on a specific task or goal (Mohammed \& Nadkarni, 2011; Standifer et al., 2015). So, without common ground, learning processes may lead to frustration and disagreements, which prevent teams from achieving increasing levels of performance over time.

The finding that team members need to have similar mental models in order to benefit from team learning is in accordance with a resource allocation perspective on team functioning (Barnes et al., 2008; Kanfer \& Ackerman, 1989). Team learning is a resourceintensive process that detracts from core task performance and that consumes time and cognitive resources (Bunderson \& Sutcliffe, 2003a, 2003b). Team members have to divide their resources between exploitation activities and exploration activities (March, 1991). When team members have similar mental models, their core task performance will run smoothly (Mohammed et al., 2010) and they will consequently have spare resources that can be used for exploration. In addition, their learning activities will require few resources as team members will be able to effectively communicate ideas for process improvement (Fussel \& Krauss, 1989) and to optimally time and synchronize their improvement-directed interactions (Mohammed \& Nadkarni, 2014). In contrast, when team members lack such common ground, engaging in team learning processes constitutes an extra effort (Bunderson \& Sutcliffe, 2003a), that may overtax the resources available to the team. Thus, for teams that do not share task and temporal mental models, it may at times be better to focus on core task performance because engaging in both exploration and exploitation may be unattainable.

Our research also advances knowledge about the differential effects of the different dimensions of shared mental models. Whereas task and temporal mental model similarity led to team performance improvement when combined with team learning behaviours, team mental model similarity did not. A possible explanation could be that the three dimensions of mental models did not develop at the same pace and that the development of one mental model dimension may influence the development of the other dimensions (Maynard \& 
Gilson, 2014). In contexts where team members need to work on the task from the early beginning of the team lifecycle and where deadlines are particularly important for team performance, team members may neglect the interpersonal aspects of teamwork, such as communication, trust, and mutual support. Therefore, the development of temporal and task mental models may precede and influence the development of team mental models and the team mental models may not yet be fully crystallized at the middle of the team lifecycle. Alternatively, teams may require a shared understanding about the team, the task, and the temporal aspects of work at different moments in the team's lifecycle (Hackman \& Wageman, 2005). For instance, it could be that team mental model similarity may be more important at the beginning instead of halfway the team lifecycle.

It is also possible that agreement among the team members on the team dimension is less crucial for team learning than agreement on the task and temporal dimensions. Team members may improve their task performance and discuss with each other despite some divergence in their understanding regarding how they should interact with each other. As learning behaviours often specifically focus on how the members can improve their interactions, team mental models may be more typically modeled as an outcome instead of a facilitator of team learning. So it may be more fluid than task and temporal mental models as it changes when team members develop new insights and procedures (Cannon-Bowers et al., 1993). In addition, extant research suggests that when interaction patterns are overtly rigid, this may actually hinder teams in adapting to novel events (e.g., Stachowski, Kaplan, \& Waller, 2009). In accordance with this notion a recent study found task mental models, but not team mental models, to have a positive effect on team performance (Guchait \& Hamilton, 2013). Another explanation for the divergent finding regarding team mental models may be related to the outcome variable under consideration. Although the team dimension of mental models has been related to team performance (Cannon-Bowers et al., 1993; Mathieu et al., 2000; Santos \& Passos, 2013), this dimension may be more important for the translation of team learning behaviours into affective outcomes, such as satisfaction and viability (Hackman, 1987).

Finally, our research emphasizes the importance of longitudinal studies for analysing team dynamics over time. Researchers have posited that longitudinal studies are needed to understand "what happens in teams" (Roe et al., 2012) or "when things happen" (Sonnentag, 2012). Longitudinal studies are needed to analyse the dynamic interrelations between coevolving constructs and to capture how teams form and develop, function, and perform 
Chapter 6

over time. Although "teams can neither be defined nor understood without reference to time" (Roe et al., 2012, p. 630), researchers have still mainly analysed teams in a static way. The present study takes a temporal perspective by analysing how team performance increases over time and which variables explain this increase (Ployhart \& Vandenberg, 2010; Roe, 2008). So far, researchers have analysed the effects of team learning behaviours on average team performance or team performance at the end of the team lifecycle; however, this approach may misrepresent the true effects of learning over time. For instance, high initial team performance may confound the relationship between learning processes and average performance. Thus, our study points to the possibility that the relationships found between variables in cross-sectional analysis may not hold when we look at dynamic trajectories of the outcome variable over time.

\section{Practical implications}

It becomes increasingly important for organizations to ensure that teams are able to constantly learn and improve their performance. Teams play a crucial role in helping organizations to compete with other organizations and to survive in challenging environments. Our results suggest that from the beginning of the team lifecycle, members need to share an understanding about the task and temporal aspects of their work in order to translate team learning processes into performance growth. Previous research suggests that the development of such common ground may be facilitated when teams engage in planning sessions or develop team charters prior to team performance and when they engage in debriefings after performance episodes (Mathieu \& Rapp, 2009; Stout et al., 1999; Vashdi, Bamberger, \& Erez, 2013). Although when left to themselves, teams often do not take the time to explicitly discuss issues, such as how the task needs to be accomplished in terms of procedures and practices, what deadlines they will need to accomplish the tasks, and at what pace activities should take place, this may actually be crucial for developing a shared understanding and improving performance in the long run (Cannon-Bowers et al., 1993; Gevers et al., 2006; Standifer \& Bluedorn, 2006). Team leaders can play an important role, as well, in promoting shared mental models and effective team learning behaviours (Edmondson, 1999; Marks et al., 2000). Team leaders play a crucial role in stimulating team members to reflect about the processes they use to accomplish the tasks, to seek and give feedback, and to discuss errors and unexpected outcomes (Schippers et al., 2008). 


\section{Limitations and directions for future research}

A limitation of the present study was that team learning processes and shared mental models were measured only once around the middle of the team lifecycle. Although prior research has shown the importance of mid-points in team projects for team development (Gersick, 1988), it could be important to measure both variables repeatedly in order to trace how the (co)evolution of team learning behaviours and mental models impact team performance outcomes. Moreover, we cannot be sure if we optimally timed our measure of team learning processes; it is possible that the effectiveness of these processes decrease from the middle of the team lifecycle. Effective team learning processes at the beginning may promote the positive trajectory of team learning outcomes because teams need to establish a solid foundation from the early stages in order to promote effective team processes and performance (Ericksen \& Dyer, 2004; Mathieu \& Rapp, 2009). Therefore, future studies could benefit from a more fine-grained measurement of team learning processes and mental models at different moments in the team lifecycle.

In this study, team learning outcomes were inferred from changes in team performance. Although this is a common way to assess team learning outcomes, an alternative approach could be the assessment of changes in team knowledge behaviours and attitudes (Kozlowski \& Ilgen, 2006; Kraiger, Ford, \& Salas, 1993). Future studies should analyse changes in team knowledge that show that team learning has occurred, such as the development of technical or motor skills, the decrease of errors, and the increase of automaticity in teams (Kraiger et al., 1993).

Finally, a limitation may be that we focused on the quality of team decisions, and not on the speed. In many contexts, decision speed may constitute a crucial component of team performance. As similarity in temporal mental models may have an impact particularly on the speed with which teams make decisions, future studies should not only analyse the accuracy but also the timeliness of team decision making (e.g., whether they submit their decisions long before the deadline, or a short time before the deadline) (Beersma et al., 2003). 
Chapter 6

\section{CONCLUSION}

Research on team learning processes has developed in relative isolation from research on team learning curves (Edmondson et al., 2007). Although many studies implicitly assume that when teams engage in learning processes, this will have a positive impact on the trajectory of team performance, an empirical study using longitudinal performance data to test this notion was lacking. Interestingly, the current study shows that team learning processes do not automatically lead to performance improvement. Our findings suggest that in order to achieve an increase in team performance over time, teams need to complement their team learning behaviours with shared task and temporal mental models. These findings stress that a strong cognitive foundation is crucial for teams in order to hone their skills and maintain competitive over time. 


\section{Chapter 7}

CONCLUSION 


\section{INTRODUCTION}

Teamwork has been pointed out by researchers and practitioners as one of the best solutions to accomplish cognitively and intellectually complex tasks because teams have a high capacity to process, store and use information to solve problems and make multiple decisions in a short period of time (Cannon-Bowers et al., 1993; Hinsz et al., 1997; Salas \& Fiore, 2004). The increasing complexity of their tasks has challenged teams and team leaders to use their cognitive resources to perform the tasks and accomplish their goals successfully (Salas \& Fiore, 2004; Salas et al., 2012). Teams need to integrate their individual mental representation of knowledge into a shared representation that helps them to engage in team processes and perform the tasks effectively, while they are pressured by time to meet the deadlines. In this thesis I argued that shared mental models and shared temporal cognitions constitute an important initial condition to ensure that teams achieve their goals; play a crucial role in facilitating team processes and team effectiveness over time; and are related to different temporal constructs.

Although progress has been made in shared mental models and shared temporal cognitions research, a number of questions remain unanswered. Empirical studies have demonstrated that shared mental models and shared temporal cognitions promote team effectiveness (Gevers et al., 2006; Marks et al., 2000; Mathieu et al., 2010). However, it is unknown in which moment of the team lifecycle, shared mental models and shared temporal cognitions are particularly important to promote team processes and team effectiveness.

Further, most of the researchers have analyzed the direct effect of shared mental models and shared temporal cognitions on team effectiveness. A small number of researchers have analyzed their effects on team processes, such as coordination and communication (Marks et al., 2002; Mathieu et al., 2010). The effect of shared mental models and shared temporal cognitions on other functional team processes, such as team creativity and team learning, and on dysfunctional team processes, such as intragroup conflict, is unknown.

Finally, time-related aspects have not been integrated into team research, and in particular into team cognition research. First, researchers have mostly analyzed the task and team dimensions of shared mental models. Although teams also need to share an understanding about the temporal aspects of work, research on temporal mental models is scarce. Second, researchers have analyzed task, relationship, and process conflict. Teams are increasingly pressured by time, need to manage multiple projects at the same time and need to 
meet tight deadlines, which may lead to conflicts about temporal issues. Nevertheless, researchers have neglected temporal conflict, and, therefore, the effect of shared mental models and shared temporal cognitions on this type of conflict in unknown. Third, team leaders play an important role in helping team members to coordinate their work, manage the time available to the tasks, and meet the deadlines, by employing temporal leadership behaviors. Despite the importance of temporal leadership, a small number of researchers have analyzed its effects on team effectiveness (Maruping et al., 2015; Mohammed \& Nadkarni, 2011). What is more, it is unknown whether teams may use their cognitive structures to avoid temporal conflict and accomplish their goals when team leaders do not employ temporal behaviors.

These gaps guided this thesis. The main goal was to analyze the effect of shared mental models and shared temporal cognitions on team effectiveness over time. In order to accomplish the main goal, three subgoals were defined. First, this thesis aimed to analyze shared mental models and shared temporal cognitions as basic conditions to facilitate fruitful team functioning. Second, this thesis aimed to analyze the effect of shared mental models and shared temporal cognitions in stimulating teams to engage in functional team processes, in avoiding dysfunctional team processes, and in performing tasks successfully. Finally, this thesis aimed to integrate time-related aspects into team cognition research (temporal mental models and shared temporal cognitions), to analyze the effect of shared mental models and shared temporal cognitions on a time-related team process (temporal conflict), and analyze shared temporal cognitions as a substitute for a time-related leadership role (temporal leadership). Related to this last goal, this thesis also aimed to analyze the effect of shared mental models and shared temporal cognitions on team effectiveness over time in two ways: analyzing shared mental models and shared temporal cognitions, team processes and team effectiveness in different time moments; and analyzing the combined effect of shared mental models and team learning on team performance over time.

In this final chapter, I discuss the theoretical and practical implications of this thesis. Then, I present limitations of this thesis and directions for future research. I finish this thesis with a brief conclusion about the main contribution of this work. 
Chapter 7

\section{IMPLICATIONS}

\section{Theoretical implications}

Table 7.1. presents the main findings of this thesis related to three aspects of team research: shared mental models and shared temporal cognitions; team processes; and temporal research. In this section, I discuss the theoretical implications of the main findings of this thesis to these three research areas. 


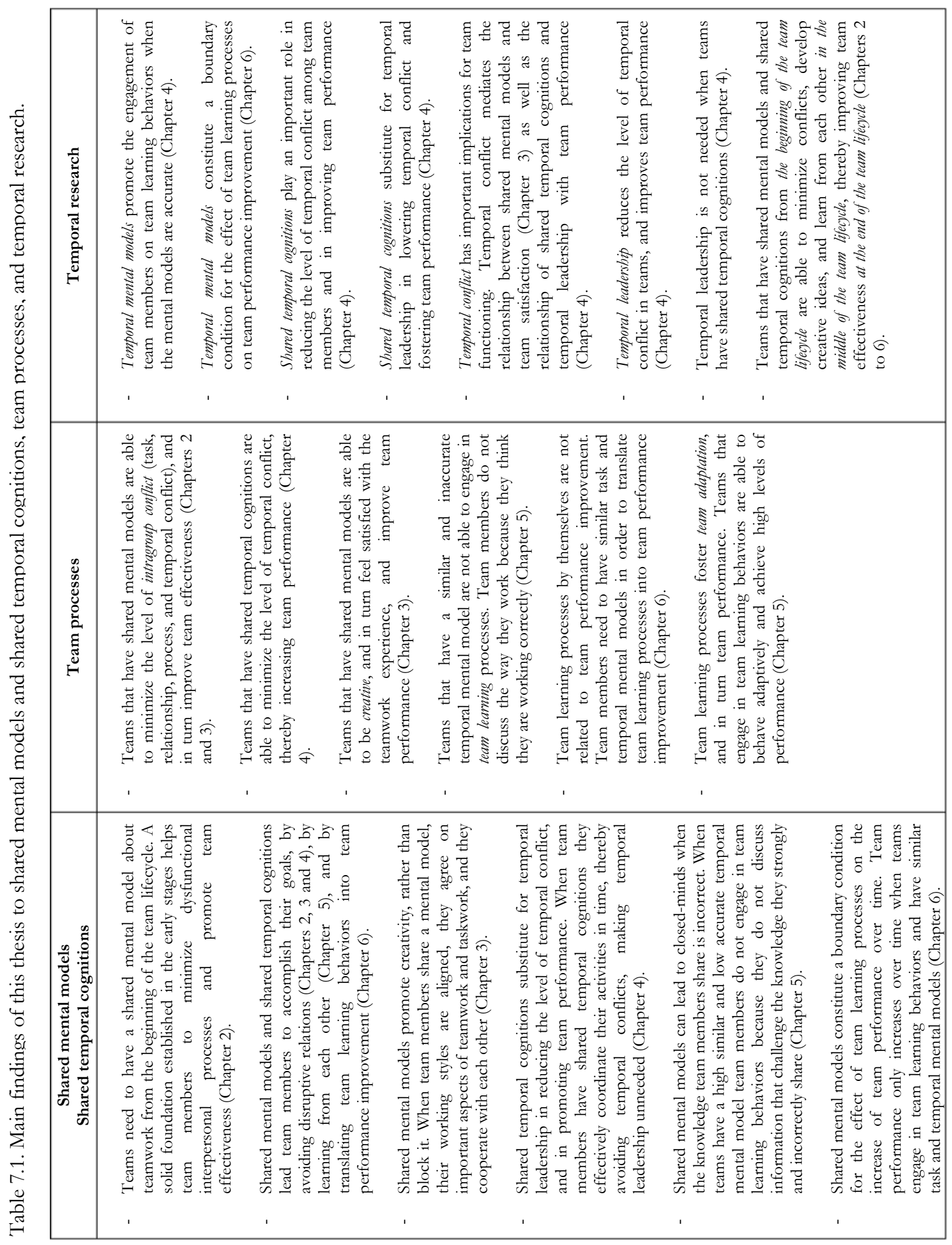




\section{Shared mental models and shared temporal cognitions}

A number of models and frameworks postulate that shared cognitions are important predictors of team effectiveness (Kozlowski, Watola, Jensen, Kim, \& Botero, 2009; Salas et al., 2005; Salas et al., 2009). However, it is not clear when (throughout the team lifecycle) shared mental models and shared temporal cognitions are particularly important for facilitating team effectiveness. This thesis demonstrates that both shared mental models and shared temporal cognitions are important from the beginning of the team lifecycle as basic conditions to facilitate fruitful team functioning. Mathieu and Rapp (2009) argue that little is known about how events or activities that occur early in the team lifecycle promote team performance over time. The findings of the study reported in Chapter 2 show that teams need to build a shared mental model about the important aspects of teamwork from the beginning of the team lifecycle to be able to minimize relationship conflict in the middle of team lifecycle and improve team effectiveness at the end.

In addition, Hackman (2012) posited that researchers should analyze "the conditions under which groups chart their own courses" (p. 435) and that help team members to achieve the desired goals. All the studies presented in this thesis show that shared mental models and shared temporal cognitions are a basic condition that is required to facilitate team members' engagement in team processes, and the accomplishment of team's goals. Although in most of the studies shared mental models and shared temporal cognitions were measured at the middle of the team lifecycle, the findings reveal that when teams build a shared understanding about the important aspects of work, they are able to minimize intragroup conflict, to be creative, to learn from each other, and to achieve their goals successfully. This means that a solid foundation related to task strategy and procedures, to team members' skills and interaction dynamics, and to the temporal demands of the work is crucial to prevent team members from engaging in conflicts, and to promote creativity and learning behaviors, thereby improving team performance, viability, and satisfaction. Thus, shared mental models and shared temporal cognitions help the team to scrutinize the important factors that can ensure that team members are able to interact with each other in an appropriated way "right from the start" (Ericksen \& Dyer, 2004).

Research on shared mental models and shared temporal cognitions has mainly focused on the positive effect of those two forms of team cognition on team effectiveness. A number of empirical studies have shown that when team members have a shared mental 
model, they are able to align their working styles and to coordinate their work, leading them to trust each other, to discuss errors, discuss new ways of working, and implement new ideas that are in accordance with the tasks and the team needs (e.g., Hülsheger et al., 2009; Mathieu et al., 2010; Resick et al., 2010). The research reported in this thesis advances knowledge about the beneficial effects of shared mental models by showing that they minimize the level of dysfunctional conflicts and in turn improve team effectiveness. Further, teams that have shared mental models develop creative ideas and in turn feel satisfied with the teamwork experience and achieve high levels of performance. In the study reported in Chapter 6 we looked at shared mental models as a contextual factor and the findings also suggest that shared mental models are beneficial for team functioning. Specifically, the findings suggest that teams need to have a common ground because team performance only increases over time when teams engage in team learning processes and have similar task and temporal mental models.

However, shared mental models may not always be beneficial for team functioning (Salas et al., 2005; Skilton \& Dooley, 2010). The conditions under which shared mental models may lead to detrimental effects have been scarcely analyzed. The research reported in this thesis clarifies this aspect by showing that a similar and inaccurate temporal mental model impedes team members to engage in team learning behaviors. When team members have such similar but incorrect models, they ignore new ideas and suggestions and do not discuss the errors they make or the problems that occur over the team lifecycle because they think the way they work is correct and appropriated to accomplish the goals. This finding suggests that shared mental models may be harmful for team functioning, leading to close-minds, when the knowledge team members share is incorrect. Thus, in order to engage in team learning behaviors, team members need to share knowledge that is correct (i.e., accurate mental models).

Finally, despite empirical studies have shown that shared temporal cognitions are relevant for efficient team functioning, they may not be important in all situations. The findings reported in this thesis demonstrate that shared temporal cognitions and temporal leadership may compensate for the lack of the other. This means that when team members share strong temporal cognitions, they do not rely on the team leaders for guidance regarding temporal issues, and vice-versa. Researchers have proposed individual-level factors that substitute for leadership, such as, subordinates' ability, experience, training, and motivation (Nübold et al., 2013; Podsakoff et al., 1996); however, it is not yet known what team-level factors may serve as substitutes for leadership. Further, research has focused on the 
relationship between team cognition and explicit coordination mechanisms, neglecting implicit coordination mechanisms (DeChurch \& Mesmer-Magnus, 2010). The findings of the study reported in Chapter 4 suggest that shared temporal cognitions, as an implicit coordination mechanism, substitutes for temporal leadership, as an explicit coordination mechanism, in reducing temporal conflict and benefiting team performance. This means that shared temporal cognitions play an important role in teams when leadership is weak or absent because team members use the shared understanding about the temporal aspects of the teamwork to perform the tasks using their time in the best way possible and avoiding misunderstandings and conflicts. Thus, these findings suggest that an implicit coordination mechanism may compensate for the lack of an explicit coordination mechanism, and vice versa, in lowering temporal conflict.

\section{Team processes}

Wildman and colleagues (2012) stated that more research is needed to analyze the team processes that are influenced by team cognitive constructs and how this relationship translates into team performance. Over the years, a number of researchers have analyzed the effect of shared mental models on team processes and consequently on team performance (Marks et al., 2000, 2002; Mathieu et al., 2000, 2010). These researchers have analyzed the mediating effect of coordination and cooperation between shared mental models and team performance. Nevertheless, other team processes may explain why both shared mental models and shared temporal cognitions improve not only team performance, but also team satisfaction and viability. This thesis advances knowledge on team processes by focusing on intragroup conflict, team creativity, team learning, and team adaptation.

Our primary contribution for the team processes literature lies in identifying intragroup conflict (a dysfunctional team process) as an important mediator in the relationship of shared mental models and shared temporal cognitions on team effectiveness. Thus far, researchers have mainly analyzed the effect of shared mental models and shared temporal cognitions on functional team processes. This thesis provides empirical evidence for the temporally based framework by Marks and colleagues (2001) showing that, indeed, emergent states influence team processes and in turn more distal team outcomes. The findings of the studies reported in Chapters 2 and 3 suggest that when team members share a mental model they are able to avoid disruptive interpersonal relations and in turn accomplish the tasks 
successfully, feel satisfied with the teamwork experience, and are willing to work with the same team in the future. In addition, the findings of the study reported in Chapter 4 indicate that shared temporal cognitions diminish the level of temporal conflict and benefit team performance. In sum, the findings of this thesis indicate that when teams have shared mental models and shared temporal cognitions, they experience positive interpersonal relationships because they are able to coordinate their work, and they are focused on task accomplishment, avoiding discussions about task procedures, personal issues, task delegation, and temporal issues. Thus, as long as teams share a common understanding about relevant aspects of teamwork, team members are likely to communicate in an appropriated way and trust in each other, thereby experiencing positive interpersonal relations and feeling safe in sharing ideas and asking questions (Kozlowski \& Ilgen, 2006; Marks et al., 2001).

Our findings also advance knowledge on team processes analyzing the mediating role of team creativity between shared mental models and team effectiveness. The effect of shared mental models on creativity has not been clear in the literature. While some authors argue that team members that have shared mental models are less likely to discuss different points of view and be creative (Skilton \& Dooley, 2010), other authors argue that team members that have shared mental models are more likely to be creative because they coordinate their work, cooperate with each other, and trust each other (DiLiello et al., 2011; Hülsheger et al., 2009). The findings of the study reported in Chapter 3 indicate that shared mental models do not block creativity; they facilitate it. Indeed, teams that have shared mental models develop creative ideas, thereby improving team performance and satisfaction. Creativity research suggests that team members are willing to be creative when the teamwork environment favors creativity - when team members are open to listen and discuss new ideas, and when team members challenge each other in a constructive way (DiLiello et al., 2011). The findings of the study reported in Chapter 3 suggest that team members also need to have a shared understanding about the important aspects of teamwork in order to be able to develop creative ideas, and in turn achieve high levels of performance and feel satisfied with the teamwork experience.

Kozlowski and Ilgen (2006) noted that greater attention needs to be dedicated to the antecedents that boost team learning. Theoretical models and frameworks of team adaptation have considered shared mental models as important boosters for team learning (Burke et al., 2006a; Rosen et al., 2011). However, empirical studies that analyze the effect of shared mental models on team learning are lacking. In particular, it is not clear when shared mental models 
promote team learning, and whether the joint effect of shared mental models and team learning facilitate team performance improvement. On the one hand, this thesis contributes to the team processes literature by showing how shared mental models relate to team learning processes. The findings of the study reported in Chapter 4 suggest that as long as team members have an accurate temporal mental model, they engage in team learning behaviors regardless of the level of similarity. However, team members that have a similar and inaccurate mental model are not able to engage in team learning behaviors. This means that in order to engage in team learning processes, teams do not need to share knowledge about the temporal aspects of work, but their knowledge needs to be correct. In sum, the accuracy of temporal mental models is an antecedent to team learning processes. On the other hand, looking at shared mental models from a different perspective - as a contextual factor - the findings of the study reported in Chapter 6 indicate that shared mental models constitute a boundary condition for the effect of team learning behaviors on the development of team performance over time. Thus, teams need to have a common ground because team performance only increases over time when teams engage in team learning processes and have similar task and temporal mental models.

Finally, this thesis advances knowledge on team adaptation as a team process. The recent models of team adaptation postulate that teams that engage in team learning behaviors are able to evaluate past performance episodes, to adapt to the changes that occur in the teams' environment, and to accomplish their tasks (Burke et al., 2006a; Rosen et al., 2011). Nevertheless, empirical studies that analyze the effect of team learning on team adaptation, and in turn on performance are lacking. Although the research reported in this thesis does not analyze the relationship between shared mental models and team adaptation outcomes, the findings of the study reported in Chapter 5 indicate that teams that engage in team learning behaviors engage in adaptive behaviors, thereby improving team performance. These findings contribute to research on team processes and to the scarce research on the antecedents of team adaptation (Maynard et al., 2015) by showing that teams that learn from each other are able to adapt their behaviors to changing situations and, consequently, accomplish the team goals. 


\section{Temporal research}

Taking into account that teams are increasingly pressured by time, work on tight deadlines, and often work on different projects at the same time, it is crucial to understand how teams can face temporal challenges. Researchers have called attention to the need to integrate time in intragroup conflict (Mohammed et al., 2009), shared cognition (DeChurch \& Mesmer-Magnus, 2010; Mohammed et al., 2012), and leadership literature (DeChurch et al., 2010; Shamir, 2011). This thesis advances knowledge on temporal research by analyzing temporal mental models and shared temporal cognitions, temporal conflict, as well as temporal leadership. This thesis also advances knowledge on temporal research by analyzing the effect of shared mental models and shared temporal cognition at the beginning of the team lifecycle on team processes in the middle of the team lifecycle, and in turn on team effectiveness at the end of the team lifecycle.

A number of researchers have called attention to the need to analyze the temporal dimension of shared mental models (Standifer \& Bluedorn, 2006; Mohammed et al., 2009), which was identified as a "key agenda item for future research" (Mohammed et al., 2012, p. 101). In order to learn from each other and accomplish their goals on time, team members increasingly need to have a shared understanding about the temporal aspects of work deadlines for task accomplishment, the time available for each task, and the sequencing of tasks (Mohammed et al., 2015; Standifer \& Bluedorn, 2006). Nevertheless, very few studies have analyzed the temporal dimension of mental models (Mohammed et al., 2015). This thesis advances knowledge on temporal mental models by providing insight into the way they promote and hinder the engagement of team members into team learning behaviors, as well as into the way temporal mental models work as a catalyst for the translation of team learning behaviors into team performance improvement.

Researchers have suggested that shared temporal cognitions play an important role in promoting team performance and team satisfaction (Gevers et al., 2006, 2009; Standifer et al., 2015). Nevertheless, "shared temporal cognition is in a nascent stage, [and] additional conceptual and empirical research is clearly needed" (Mohammed et al., 2012, p. 96). This thesis contributes to the shared temporal cognition literature by showing that teams that have a shared understanding about temporal aspects of work are able to minimize temporal conflict and in turn improve performance. More importantly, the research reported in this thesis 
shows that shared temporal cognitions substitute temporal leadership in reducing temporal conflict and fostering team performance.

Mohammed and colleagues (2009) stated that besides relationship, task and process conflict, research on team conflict should reflect the disagreements about when tasks and work should be accomplished and in what order. Research on temporal conflict is particularly important because teams increasingly work under tight deadlines and manage multiple projects simultaneously, which may lead to disagreements on temporal issues. This thesis shows that temporal conflict is, indeed, a mechanism that negatively impacts team functioning. Therefore, studies that show how temporal conflict may be minimized are needed. The findings of this thesis advance knowledge on temporal conflict by showing that teams need to have shared mental models to avoid conflicts about temporal issues, be creative, and accomplish their tasks. Further, teams need to have shared temporal cognitions, or team leaders need to employ temporal leadership behaviors, in order to minimize temporal conflict and perform well.

Although scholars have recognized the increasing importance and pressure for time in teams and organizations, researchers have failed to integrate time in team leadership constructs (Shamir, 2011; van der Erve, 2004). The research reported in Chapter 4 incorporates temporality in terms of content into a leadership construct by analyzing temporal leadership. Temporal leadership is particularly relevant for teams that work under pressure and need to meet tight deadlines because it helps team members to coordinate their tasks, to allocate time to each task and to urge the team to finish the tasks on time (Mohammed \& Nadkarni, 2011). Nevertheless, few empirical studies have focused on temporal leadership (Maruping et al., 2015; Mohammed \& Nadkarni, 2011). This thesis provides insights about temporal leadership by demonstrating its effects in lowering temporal conflict and promoting team performance. In fact, when team leaders employ temporal leadership behaviors, team members are able to avoid temporal conflicts and accomplish their tasks. Importantly, when leadership is weak or absent, it may be substituted by shared temporal cognitions. This means that teams do not need temporal leadership when they have shared temporal cognitions because team members make sense of the similar understanding they have about the best way to use their time in order to avoid temporal conflict. In sum, both temporal leadership and shared temporal cognitions can compensate for the lack of the other.

Marks and colleagues (2001) posited that teams have distinct needs over their team lifecycle, engaging in different processes at different times depending on the phase they are in and on the characteristics of the team that emerge over time. The research reported in this 
thesis indicates that a shared understanding about the important aspects of work at the beginning of the team lifecycle is important to help team members to minimize conflict, to develop creative ideas, and to learn from each other around the middle of the team lifecycle, and in turn to achieve team's goals in the end of the team lifecycle. Thus, when teams have shared mental models and shared temporal cognitions right from the start, team members have cognitive resources available to deal and overcome the problems and challenges they face over time, to find solutions discussing with each other and to accomplish the team's goals successfully (Ericksen \& Dyer, 2004; Hackman, 2012). In addition, the findings of the study reported in Chapter 6 indicate that shared mental models are an important boundary condition to translate the team learning behaviors, in which team members engage in the middle of the team lifecycle, into team performance improvement. This means that being on the same page about the important aspects of the work has consequences over the team lifecycle.

\section{Practical implications}

The research reported in this thesis offers practical implications for organizational and business teams. In particular, these implications are oriented to team leaders that play an important role in facilitating an optimal team functioning. In addition, the research reported in this thesis offers implications to human resources managers, in particular to their human resources management and development practices.

Organizations increasingly rely on teams to perform the work and accomplish the tasks. The studies reported in this thesis reveal that, indeed, teamwork works. Team members are able to achieve the team goals, and teams improve their performance over time when they share an understanding about the important aspects of work, interact with each other and learn from each other. This means that having a team based structure helps organizations to accomplish their objectives and to remain competitive. Therefore, when the tasks are complex and challenging, team leaders should put some employees working together in order to perform the tasks.

However, as Thompson (2004) stressed, "putting people into teams does not solve problems; if not done thoughtfully, this may even cause more problems" (p. 11). Teams are more than a collective group of people that work individually on the same place, on individual sub-tasks, and that in the end merge the different sub-tasks. Teams need to exchange ideas, experiences, and coordinate their efforts to accomplish the tasks. Importantly, a number of 
guidelines need to be followed in order to increase the likelihood to build a functional and productive team. First of all, team leaders need to select the most appropriated people to work together and they need to communicate to team members who is part of the team and who is not. Knowing who the team members are ensures that they interact, communicate, and coordinate their work with the appropriate people.

Further, team leaders need to build a team composed of people who prefer to work within a team than alone. People who like to work within a team are able to contribute with their knowledge and skills, communicate with each other, coordinate tasks and make efforts according to the team-level goals and interests. By contrast, people who prefer to work alone are not willing to contribute with their knowledge and skills, are worried with what other team members achieve or could achieve with the knowledge shared within the team. As individualist people prefer to work and make efforts to achieve their own personal interests and goals than the team-level ones, they are likely to create problems and conflicts within the team.

Team leaders also need to transmit clearly the main goals of the teams because team members need to know, understand and agree about them. The goals need to be challenging to ensure that team members are motivated to accomplish them. Further, team leaders need to choose people with diverse skills and competencies. Teams need to be composed of members who have different technical skills that allow them to perform the tasks and accomplish the goals. Team members also need to have interpersonal skills that help them to communicate and interact with each, manage and avoid conflicts, discuss problems that occur, and provide appropriated feedback that help the team to face unexpected situations that may occur.

Importantly, team leaders need to provide appropriated time and space to team members understand the tasks they need to accomplish, to know each other, to know each other's skills, competencies, and preferences, and to understand the deadlines they need to meet. Finally, in order to function correctly, a team needs to have someone who plays the leader's role. Team leadership behaviors may be employed by a formal leader (i.e., who is formally assigned to lead the team) or by an informal leader (i.e., someone that emerges or is chosen informally by the team members), or may be shared among the team members (i.e., multiple team members may employ leadership behaviors). Alternatively, as the study reported in Chapter 4 indicates, team members may use the knowledge structures they possess to guide their behaviors when team leadership is weak or absent. 
The research reported in this thesis is particularly important for team leaders that are interested in understanding how teams perform over time and in helping the teams to perform over their team lifecycle. Teams have different needs at different time moments, and consequently, team leaders should provide differentiated support to teams depending on the phase they are. At the beginning of the team lifecycle, team members need to work together to build a common ground about the important aspect of work that will guide the team functioning and effectiveness over time. In other words, team members need to build task-, team- and temporal shared mental models and shared temporal cognitions. As teams often do not take time to discuss the issues that lead them to have a shared knowledge when they are left to themselves, team leaders may play an important role in facilitating team members to discuss with each other. Team leaders may help team members to discuss and agree about: the best strategies and tools to accomplish the tasks; the best way to distribute the work among the team members according to their skills and preferences; and the best way to distribute the time for the tasks, meeting the deadlines. Thus, team leaders may play a crucial role in facilitating a shared understanding about these aspects, which will promote team members' engagement in team processes, as well as, team effectiveness.

After the establishment of a common ground, team members engage in different team processes, around the middle of the team lifecycle, that help them to achieve the team goals later on. In this phase, team leaders may help the team to avoid disruptive situations, such as conflicts, and to take advantage of beneficial situations, such as discussions about mistakes, new ideas, and new working methods. Regarding intragroup conflict, team members may be instructed about strategies to avoid or manage conflict, and team leaders may also provide team members with tools that help them to avoid and to manage conflicting situations.

Team leaders may promote the discussion of different ideas, ensuring that all team members express their points of view, in order to achieve consensus and do not engage in task conflict situations. In order to prevent relationship conflict, team leaders may instruct team members to focus on the content of the arguments and to not take disagreements in a personal way. Team leaders may help to prevent process conflict by allocating responsibilities among the team members, and by providing team members with autonomy to choose the specific tasks they want to execute according to their preferences or knowledge. Finally, team leaders may help team members to prevent temporal conflict by helping team members to 
achieve a consensus on the allocation of time to the tasks, and by scheduling deadlines to check whether the tasks are being completed or not.

To prevent intragroup conflict in general, team leaders (and/or team members) may create a team chart where are identified: the main goals of the team; all the tasks the team need to accomplish and the time allocated to each task; the person responsible for each task; and the deadlines for all the tasks and for the main goals. This way, team members may check the team chart throughout the team lifecycle, and update it when is need, avoiding conflict situations.

Team leaders may also challenge team members to be creative. Team leaders may stimulate team members to present creative ideas and discuss them within the team in order to decide whether they are appropriated or not. Both team members and team leaders should provide feedback on the ideas presented. This way, team members perceive that their team supports for creativity and they are likely to present new ideas over the team lifecycle because they know that their teammates and the team leaders will not criticize them, rather they will help them to improve the idea and implement it if it is appropriated.

Team leaders may also play an important role in promoting team learning behaviors. Team leaders may stimulate team members to communicate and discuss the errors they make and to find appropriated solutions to solve them in order to prevent that other team members make the same errors. Team leaders may also stimulate team members to reflect about the process and the working methods they use to accomplish their tasks, in order to improve the working procedures. Moreover, team leaders may help team members to seek and ask for feedback from each other and from external people because it may help them to change the way they work accordingly. Finally, team leaders may stimulate team members to carefully listen the ideas of each other, elaborate these ideas, complement with their own ideas and then collectively make decisions based on the ideas the team discussed. Promoting team learning behaviors help team members to adapt their strategies to unexpected events and to achieve their goals.

When team members do not easily engage in team learning behaviors team leaders may schedule a time period, once a week for instance, to "oblige" team members to reflect about the way they have worked, to discuss errors they might have done, and to give and ask for feedback. Importantly, as the study reported in Chapter 5 demonstrates, team members need to share accurate knowledge about the temporal aspects of work to engage in team learning behaviors. Therefore, team leaders should schedule regular meetings throughout the 
team lifecycle to ensure that the way team members think about when tasks need to be done, and how much time they have to work on each task, is not only aligned among the team members, but is also correct according to team leaders or task experts' understanding. In those meetings, it may be monitored what team members have been done and when, what they still need to do in the time they have, and may be discussed the best way to use the time left in order to accomplish the tasks and meet the deadlines.

Regarding the human resources management and development practices, this thesis offers implications for training of team members and team leaders, as well as for recruitment and selection, and performance evaluation system.

Concerning training programs, team members and team leaders may be instructed about practices and strategies to help them to develop shared mental models and shared temporal cognitions, to manage and avoid intragroup conflict, to facilitate creative ideas, and to engage in team learning behaviors. Importantly, team members and team leaders should be trained as a team, and in the context in which the team operates. Team members develop cognitions and engage in behaviors collectively while they are executing their team task. Therefore, team learning is a collective product that is more than the sum of the individual learning of the team members. When team members are together it is easy to think about the way they work, the knowledge they have and share, and the way they think about the important aspects of work. Therefore, it is preferred to train team members within the context of their own work environment and including the complete team. A training program for only one team, in the context where the team operates, and according to the team needs, allows team members to receive specific and useful information, acquire competencies and practice them, and to receive and ask for feedback. Importantly, the team leader should participate in the training with the team members, and should be supportive of training. Team leader participation facilitates the transfer and usage of knowledge and competencies in the work context, and facilitates a supportive organizational climate (Gregory, Feitosa, Driskell, Salas, \& Vessey, 2013).

In training programs about shared mental models and shared temporal cognitions, team members and leaders should be instructed about the importance of these cognitive aspects, and with some strategies to develop them. Before team members start to work on the tasks, they should participate in a planning session in which the team leader plays the moderator role. Team members and the team leader should discuss and clarify what is the main goal of the team, who is responsible for what, what are the main competences and 
preferences of each team member, how much time they have to work on the tasks, and when the deadlines are. Then, a mind mapping intervention may be used to facilitate the development of shared mental models and shared temporal cognitions (Rentsch et al., 2010). First, team members, individually, should think about the main actions they need to do and the main information they need to get to accomplish the task. They may write this information on a paper. Then, team members should explain to each other the meaning of the main actions and information, and why this is important to accomplish the team goals. After that, team members, collectively, should create a mind mapping by making the connections among the main actions and information, and between these main information and team performance. Teams may make the mind mapping in a cardboard or in a white board, for instance, and make it visible to all the team members. This way, team members may check the mind mapping whenever possible and behave accordingly. When team members are geographically dispersed, they may discuss the mind mapping by means of a video-conference tool and create it in software that allow them to online share and check the mind mapping whenever they want. As shared mental models need to be accurate, team leaders and/or task experts need to participate in the discussion to create a mind mapping. If this is not possible, team leaders and task experts should check and validate the mind mapping later and discuss with the team their ideas and suggestions to improve it. This planning session where team members create a mind mapping aims to promote a shared understanding among them about the task, team, and temporal aspects of work, by aligning their strategy according to each other skills and to task experts' knowledge.

Some strategies to manage and avoid intragroup conflict, to facilitate creative ideas, and to engage in team learning behaviors were discussed and presented in the previous paragraphs where we discussed how team leaders may help team members throughout the team lifecycle. Importantly, team leaders by themselves may not be aware of those strategies and may not be able to transmit those strategies properly to team members. Therefore, training programs about conflict management strategies should to be offered to team members and team leaders before they start to work together on task accomplishment. These training programs allow team members and leaders to develop conflict management competences that help them to prevent conflicting situations and to achieve their goals. Training programs about strategies to develop creative ideas and to engage in team learning behaviors should also be offered to team members and leaders. In these training programs team members and leaders may be instructed about the importance of listening new ideas and 
new working methods, discussing errors and unexpected outcomes, and complementing each other's ideas in order to develop creative ideas, engage in team learning behaviors and achieve team's goals.

Training programs about the importance of the scheduling, synchronization, and the allocation of time for each task should also be offered in organizations, in particular to team leaders. Temporal leadership behaviors are increasingly important because teams need to work under a lot of time pressure, meet very tight deadlines, and work on different projects simultaneously. Thus, team leaders should be instructed about the best way to help team members to prioritize the tasks, to understand when they need to start to work on a task and when they need to finish it by communicating the temporal aspects of work to team members and monitoring them over time. A training program about these aspects help team leaders to effectively communicate the important issues to team members, thereby helping team members to temporally coordinate their work, achieve their goals and meet the deadlines.

Another technique that aims to promote learning from experience, and that can be used to improve team effectiveness by building teamwork skills and competencies refer to debriefings. Debriefings are sessions in which team members "(a) reflect upon a recent experience, (b) discuss what happened, (c) identify lessons learned and opportunities for improvement, and (d) plan for the future (Tannenbaum, Beard, \& Cerasoli, 2013, p. 489). Debriefings last thirty minutes or less, and may be led by the team leader or by an external person, for instance, an instructor. Debriefings present a number of advantages over team building and team training as they are "less expensive, less time-consuming, and easier to employ" (Tannenbaum et al., 2013, p. 490). Tannenbaum and colleagues (2013) identified six types of debriefings. For instance, debriefings may be conducted after a unique work experience, in which team members reflect and learn from experience and plan the future (i.e., debriefing a work experience); may be conducted repeatedly over time after work experiences to ensure that team members apply the lessons learned and that they do what is supposed to do, and achieve their goals (i.e., ongoing debriefing of work experiences); and may be combined with team training - debriefings are conducted after a team training exercise to maximize the competencies acquisition (i.e., debriefing a training experience).

Like team training, debriefings can be used to help team members and team leaders to deal with interpersonal conflicts, to develop shared mental models, and to learn from each other, being the ultimate goal improve performance. Nevertheless, debriefings are likely to be more effective than training in facilitating a fruitful teamwork environment and in promoting 
team performance. Debriefings may be conducted repeatedly over the team lifecycle whenever the team needs, or in pre-scheduled moments (e.g., after each performance episode), while team training is less frequent over the team lifecycle because it demands an instructor and a previous agreement between the team/organization and the instructor. Indeed, a team may start and finish a project without engage in a team training session. The team leader and all the team members should be involved in the debriefing sessions. Nevertheless, team leader plays an important role in ensuring that debriefing sessions are effective. Thus, team leader (or another facilitator) needs to structure the session to ensure that the team is focused, to prepare himself/herself to lead debriefings effectively, to develop the discipline to debrief, and to ensure that there is good quality information to share (Tannenbaum et al., 2013). Debriefings may be used for team leaders to "oblige" team members to reflect about their work on previous tasks, as well as to align the way team members think about task, team, and temporal aspects of work.

The research reported in this thesis provides two strategies to human resources management regarding the recruitment and selection process. First, when human resources managers initiate a recruitment and selection process and know that the selected people will work mainly on teams, they should select team-oriented people. Individuals that prefer to work within a team and that are predisposed to coordinate and combine their information with the task inputs of other team members are able to make efforts in favor of team-level goals and interests, rather than individual-level ones. Thus, human resources managers should evaluate the team orientation level of the candidates that will work on teams. Second, human resources managers should select people that have similar understanding about the general functioning of a team when compared with other team members, team leaders or task experts. If the human resources managers need to select all the people that will work together as a team, they may evaluate the individual mental models of all the candidates and check whether they are similar or dissimilar among them. Human resources managers may also compare the individual mental models of the candidates with the mental models of the team leaders and/or task experts. If the human resources managers need to select just one person to join a team, they may evaluate the mental model of this person and compare it with the mental model of the team and with the mental model of the team leader/task expert. In both situations, human resources managers should prefer a person/people who have similar understanding about the general functioning of a team because this way it is likely to be easier to work with the other team members aligning their strategies and their working methods. 
Finally, this thesis offers an implication related to the performance evaluation system. In organizations, employees that work on the same teams should evaluate the performance of each other. Instead of a person being evaluated by the team leader, and by the peers that work with him/her in the same department, for instance, but that do not work on the same team, this person should be evaluated on his/her performance by the team leader and by the teammates of all the teams in which he/she participated. This strategy may reduce the tendency of some people to be individualist and may reduce free-riding. If a person knows that in the end of the team lifecycle is evaluated by the people that worked directly with her/him, this person may make an extra effort to perform his/her tasks, and to perform them in the best way possible. Although in the end may still be difficult for external people to the team to identify who is responsible for what, employees know that their effort and contributions will be evaluated by their teammates. Thus, employees are likely to contribute to the team goals achievement, by avoiding disruptive interpersonal relations throughout the team lifecycle, engaging in functional interpersonal and behavioral processes, and performing the tasks successfully.

\section{LIMITATIONS AND DIRECTIONS FOR FUTURE RESEARCH}

An important limitation of this thesis refers to the use of a management and strategy simulation in all the studies because the findings may not be generalizable to "real" world teams. Although the task was artificial, the teams worked together for more than five weeks (before the 5-weeks simulation teams worked together for a week to be familiarized with the simulation, materials, decision sheets, and team members). In order to run the company, making the best decisions possible, team members need to deal with complex-decision problems, and focus on multiple indicators to accomplish specific objectives while they were pressured by time. Therefore, the work environment of these artificial teams was similar to the ones of real teams in authentic organizations. However, it would be valuable to conduct a number of studies with real teams in order to understand whether the effects of shared mental models and shared temporal cognitions on team processes and on team effectiveness are stronger or weaker than with artificial teams.

It could be expected that the effects may even be stronger in organizational teams, as in the field the shared mental models and shared temporal cognitions have really been engrained in people over longer periods of time. Thus, future studies should measure shared 
mental models and shared temporal cognitions in teams performing in organizations. However, in particular for shared mental models, this can be not easily tested with the existing measures because these need to be developed specifically for each context. In order to measure shared mental models in organizations, a two-stage process may be needed: first, using interviews with subject matter experts for identifying aspects that are important within the organization, and second asking people to relate the main aspects (as we did in the studies reported in this thesis). As the structural network technique is complex and time-consuming for team members, the way similarity of shared mental models is captured could be simplified by asking people to rank the aspects in order of importance (using an importance rating technique) or by asking people to ranking the aspects from the highest to the lowest priority (using a priority ranking technique). A different possibility to measure shared mental models is through a textual analysis technique called map analysis (Carley, 1997). Using this technique, the similarity of shared mental models can be assessed from written text, by the extraction of concepts and relationships in order to determine "whether the text's authors are just using the same words or are actually exhibiting shared meaning" (Carley, 1997, p. 537). Further, the accuracy of mental models can be assessed comparing written text from managers or leaders and team members.

In the study reported in Chapter 3 shared mental models were assessed through a perceptual measure. Although this technique does not allow the evaluation of shared mental models similarity, it can be useful to measure shared mental models in contexts were it is difficult to measure them through structural network measures. Nevertheless, the four-items scale used in study 2 may be insufficient to measure all the knowledge contents of shared mental models, and may contain different ideas in the same items. For instance, item 2 contains information about the team members' responsibilities, interdependent roles, and communication patterns, which refer to three different aspects of teamwork ("In my team, the team members have a similar understanding of each other's responsibilities, interdependent roles and communication patterns"). Therefore, in the future, a more valid shared mental model measure that can be used in different contexts without the need to adapt the items should be developed. A shared mental model measure could cover all the knowledge contents identified by Cannon-Bowers and colleagues (1993) that were merged into team and task domains by Mathieu and colleagues (2000). For instance, three items could be developed to measure the three aspects mentioned above (responsibilities, interdependent roles, and communication patterns). This shared mental model measure could not include the knowledge 
about the temporal aspects of teamwork as the shared temporal cognition measure already assesses this aspect through a perceptual technique. A perceptual shared mental model measure can be easily filled in by team members from different contexts, and the feedback can be provided quickly to team members and to team leaders.

The main objective of this thesis was to analyze the effect of shared mental models and shared temporal cognitions on team effectiveness over time. Although in the studies reported in this thesis the variables were measured in different time moments, only study reported in Chapter 6 is a longitudinal one. In the study reported in Chapter 2 shared mental models were measured at two time points and their evolution was assessed. However, with only two measurement points it is not possible to assess the shared mental models trajectory because in order to assess and predict between-team variations in performance trajectories, and to conduct longitudinal analysis, at least three data points in time are required (Chan, 1998; Pitariu \& Ployhart, 2010; Ployhart \& Vandenberg, 2010). The other studies measured the variables at different points in time throughout the simulation, acknowledging "the importance of temporality while only examining differences between teams at different points in time" (Coultas, Driskell, Burke, \& Salas, 2014., p. 687). Thus, the findings do not allow the understanding of "what happens in teams" (Roe et al., 2012) and "when things happen" (Sonnentag, 2012) because the same variables were not analyzed repeatedly over time. Future studies should analyze teams in a longitudinal way, in order to analyze true causal relationships, specify the phenomena's beginning, ending, duration, and their trajectory (Roe et al., 2012; Sonnentag, 2012).

Another limitation in this thesis, and related to the previous one, is that the evolution of shared mental models and shared temporal cognitions was not assessed. This is definitely a missing topic in particular in shared mental models research. Although a number of researchers postulate that team members' mental models become more similar over time, empirical research shows that they do not become more similar over time (Levesque et al., 2001; Mathieu et al., 2000). Future studies should analyze "what happens" with shared mental models analyzing how "they unfold over time, looking at their dynamic features (onset-offset, duration, and patterns or trajectories) and their dynamic interrelations" (Roe et al., 2012, p. 639). Future research should also analyze which variables foster and inhibit the evolution of shared mental models. Importantly, as to develop a shared understanding among team members about the teamwork, taskwork, and temporal aspects of work is not easy and requires time (Cannon-Bowers et al., 1993; Levesque et al., 2001), there may be a crucial factor 
- training - that fosters the development of shared mental models over time. Shared mental models training could provide team members from different contexts with mechanisms that help them to develop an understanding about the task they need to perform and the environment in a rapid and accurate way (Cannon-Bowers et al., 1993). Shared mental models training may be based on the mind mapping intervention mentioned above (Rentsch et al., 2010). In the future, experimental studies that analyze the effect of shared mental models training (vs. control group) on shared mental models development over time and on performance should be conducted.

In this thesis the structural network metric was used to assess the shared mental models; however, social network analysis was not used to identify, describe, and explain different types of structures and relationships among individual team members' mental models (Slaughter, Yu, \& Koehly, 2009). The approach used in this thesis looks at the overall similarity within the team and does not distinguish between the relationship among the team members. Future studies should analyze shared mental models from a social network analysis perspective in order to understand in what extent the team members share a similar and accurate mental model. This technique can be used not only to analyze how the individual mental models are related. For instance, it may be important that two team members that are very interdependent have more similar mental models than two members that do not rely so strongly on each other. Social network analysis can be also used to analyze, for instance, the structure of intragroup conflict and team leadership among team members. In the future, researchers should analyze who is the most important member who is connected to the rest of the individual team members (i.e., the network), and who is the most popular team member, and who is the most distant (or close) team member from others in the network (Contractor \& Su, 2012; Espinosa \& Clark, 2014; Slaughter et al., 2009). These measures allow researchers to understand, for instance, who is (are) the most influential team member(s) to the development of a shared mental model, who has (have) the most distinct way of thinking about important aspects of teamwork, who is (are) involved in conflicting situations and with whom, and whether the formal team leader is indeed the most important and influential person of the team. Further, social network analysis allows researchers to examine whether there are subgroups among the team that may negatively influence shared mental models development or team performance.

A last limitation of this thesis refers to absence of studies that integrate different cognitive constructs. Future studies should analyze how different cognitive constructs, such as 
shared mental models, shared temporal cognitions, transactive memory systems and situation awareness, are interrelated and how they influence team processes and performance. The surgical team example in the first chapter of this thesis shows that a team may develop multiple cognitive constructs; however, studies that analyze how these multiple constructs interact and influence team functioning is scarce (DeChurch \& Mesmer-Magnus, 2010; Guchait, 2016; Salas \& Wildman, 2009; Wildman et al., 2012). The interaction of shared mental models and transactive memory systems is particularly important because the former focuses on the similarity of knowledge and the later on the distribution of knowledge among team members. It is important understand how they interact over time, and when, throughout the team lifecycle, one is more important than other. As Wildman and colleagues (2012) speculate "perhaps shared mental models and transactive memory are causally related, and as specialization of knowledge increases, the sharedness of mental models decreases, or viceversa" (p. 104). Thus, shared mental models may be more important in the beginning of the team lifecycle than transactive memory system, because team members need to be on the same page when they start. Then, as team members focus on and deepen their own specialization, the transactive memory system may be more than shared mental models to accomplish the tasks.

\section{CONCLUSION}

A shared understanding about the task, team, and temporal aspects of teamwork is important for teams performing intellectual and cognitive complex tasks under time pressure. The research reported in this thesis aimed to understand the effects of shared mental models and shared temporal cognitions on team processes and team effectiveness over time. The results of the studies reported in this thesis indicate that in order to ensure that a team is effective, team members need to have a similar and correct understanding about the taskwork, team interaction, and temporal aspects of work, which help them to avoid dysfunctional interpersonal relations and to engage in functional team processes, and in turn to achieve the team goals over the team lifecycle. Importantly, team leaders may help the team members to temporally coordinate their work, and to avoid temporal conflict, by employing temporal leadership behaviors. Nevertheless, when team temporal leadership is weak or absent, team members may make use of shared temporal cognitions to avoid temporal conflicts and to achieve their goals. 

SUMMARY 


\section{SUMMARY}

Organizations increasingly rely on teams to accomplish cognitively and intellectually complex and demanding tasks. In order to perform such tasks successfully, team members need to develop a shared representation of knowledge about important aspects of teamwork. In other words, team members need to have shared mental models - a common understanding among team members about the task, team and temporal aspects of work -, and shared temporal cognitions - a common representation of knowledge about deadlines and the use of time during the task. The main goal of this thesis is to analyze the effects of shared mental models and shared temporal cognitions on team processes and team effectiveness over time. More specifically, this thesis has three goals. First, to analyze the hypothesis that shared mental models and shared temporal cognitions are basic initial conditions that promote a fruitful team functioning. Second, to analyze the effect of shared mental models and shared temporal cognitions on functional and dysfunctional team processes and in turn on team effectiveness. Third, to integrate time in team cognition research by following two distinct approaches: (1) by analyzing time-related constructs and relating them with shared mental models and shared temporal cognitions; and (2) by measuring shared mental models, shared temporal cognitions, team processes and team effectiveness in different time moments, as well as by analyzing the combined effect of shared mental models and team learning behaviors on team performance over time.

Chapter 1 aims to contextualize the research made in this thesis. This chapter starts with definitions of teams and teamwork. The most commonly used team effectiveness models are explained, as well as the main mechanisms and factors that influence team effectiveness and that guided this work. Then, building on cognitive psychology, it is explained how individuals acquire and use knowledge, distinguishing mental models from schemas. In addition, it is explained how social and organizational researchers integrate cognitive psychology in their research, and how teams process information and build a shared representation of knowledge. Finally, shared mental models and shared temporal cognitions are conceptualized and the (un)explored mechanisms on the relationship of shared mental models and shared temporal cognitions with team effectiveness are presented.

Chapter 2 describes a study that analyzes whether similar mental models (team and task dimensions) at the beginning of the team lifecycle influence the level of relationship conflict, shared mental model similarity at the middle of the team lifecycle, and in turn team 
effectiveness (team performance, satisfaction, and viability). This study also analyzes whether shared mental models become more similar from the beginning to the middle of the team lifecycle. In the study, 92 teams (3-5 members) performed in a management simulation over five weeks. The results indicate that relationship conflict mediates the relationship between team mental model similarity at the beginning of the team lifecycle and team effectiveness at the end of the team lifecycle. Relationship conflict does not mediate the effect of task mental model similarity on team effectiveness. In addition, task mental models similarity at the middle of the team lifecycle mediates the relationship between task mental models at the beginning of team lifecycle and team effectiveness at the end of the team lifecycle. Finally, no support was found for an increase in similarity of shared mental models from the beginning to the middle of the team lifecycle.

Chapter 3 describes a study that analyzes the mediating mechanisms of intragroup conflict (task, relationship, process and temporal conflict) and creativity in the relationship between shared mental models and team effectiveness (team performance and satisfaction). In the shared mental models literature, it has been discussed whether shared mental models stifle creativity or promote it. In this study it is proposed that shared mental models facilitate team creativity because this ensures that members' working styles are aligned, which enables them to effectively coordinate their work. The model was tested in a sample of 161 teams (735 individuals) performing in a management simulation over five weeks. Findings of the study suggest that intragroup conflict mediates the relationship between shared mental models and satisfaction. However, intragroup conflict does not mediate the relationship between shared mental models and team performance. Importantly, creativity positively mediates the relationship between shared mental models and team effectiveness. Finally, intragroup conflict and creativity sequentially mediate the relationship between shared mental models and team effectiveness.

Chapter 4 describes a study that analyzes the effect of temporal leadership and shared temporal cognitions in reducing temporal conflict, and in facilitating team performance. What is more, this study analyzes whether shared temporal cognitions can substitute for temporal leadership in lowering temporal conflict. By combining two types of coordination mechanisms, this chapter postulates that an implicit coordination mechanism shared temporal cognitions - may substitute an explicit coordination mechanism - temporal leadership - in diminishing temporal conflict. More specifically, it is argued that when shared temporal cognitions are high, teams do not need temporal leadership, and that both temporal 
leadership and shared temporal cognitions can compensate for the lack of the other. The model was tested in a sample of 142 teams (650 individuals) performing in a management simulation over five weeks. Findings of the study indicate that temporal conflict mediates the relationship between temporal leadership and team performance, and the relationship between shared temporal cognitions and team performance. Moreover, findings indicate that shared temporal cognitions function as a substitute of temporal leadership in reducing temporal conflict. Thus, as expected, when shared temporal cognitions are high, there is no need for temporal leadership in order for teams to experience low levels of temporal conflict.

Chapter 5 contains an investigation of the moderating effect of temporal mental model accuracy on the relationship between temporal mental model similarity and team learning. Further, this chapter analyzes the mediating mechanism of team adaptation in the relationship between team learning and performance. It is argued that sharing an inaccurate mental model leads to closed minds. In particular, when team members have an inaccurate temporal mental model, similar mental models lead them to engage less in team learning behaviors. In the study, 68 teams (319 individuals) performed in a management simulation over five weeks. Findings indicate that when temporal mental model accuracy is high, similarity is not significantly related to team learning; while when accuracy is low, the more similar the temporal mental model is, the less team members engage in learning behaviors. Therefore, shared mental models can lead to closed-mindedness when the knowledge the team members share is incorrect. In addition, our findings suggest that team adaptation mediates the relationship between team learning and performance.

Chapter 6 describes a study that integrates a team process and a learning curve perspective on team learning. This chapter adopts a longitudinal approach by measuring team performance repetitively over time, and by using team learning processes and shared mental models to predict the temporal trajectory of team performance. More specifically, this chapter analyzes the effect of team learning processes on team performance improvement; and whether this relationship is moderated by task, team, and temporal mental models similarity. The model was tested in a sample of 67 teams (314 individuals) performing in a management simulation over five weeks. The findings suggest that team learning behaviors do not have a direct effect on team performance improvement. Temporal and task mental models are crucial for the translation of team learning behaviors into performance improvement. In particular, the findings suggest that when teams have similar task and temporal mental models, engaging in team learning processes is beneficial; whereas when teams have dissimilar task and temporal 
mental models, engaging in team learning processes is detrimental to performance improvement. The findings fail to support the moderating role of team mental model similarity.

The studies reported in this thesis highlight the important role of shared mental models and shared temporal cognitions established early in the team lifecycle for team processes and team effectiveness. In addition, the studies highlight the importance of timerelated constructs of team functioning, and the importance of analyzing teams over their lifecycle. Although more research is needed to further investigate the antecedents, consequences, and boundary conditions of shared mental models and shared temporal cognitions, the studies reported in this thesis provide avenues for future research about these two cognitive constructs. In particular, it provides important suggestions on how to analyze how shared mental models and shared temporal cognitions emerge and develop over time. This thesis presents a number of practical implications for teams, team leaders, and human resources managers. 

REFERENCES 
References

Aiken, L., \& West, S.G. (1991). Multiple regression: Testing and interpreting interactions. Newbury Park, CA: SAGE.

Allison, P.D. (1999). Multiple Regression: A Primer. Thousand Oaks, CA: SAGE Publications.

Amabile, T.M. (1988) A model of creativity and innovation in organizations. Research in Organizational Behavior, 19, 123-167.

Amabile, T.M., Barsade, S.G., Mueller, J.S., \& Staw, B.M. (2005) Affect and creativity at work. Administrative Science Quarterly, 50, 367 - 403.

Amason, A.C., \& Mooney, A.C. (1999). The effects of past performance on top management team conflict in strategic decision making. International Journal of Conflict Management, 10 , $340-359$.

Amason, A.C (1996). Distinguishing the effects of functional and dysfunctional conflict on strategic decision making: Resolving a paradox for top management teams. Academy of Management, 39, 123-148.

Ancona, D.G., \& Chong, C. (1999). Cycles and synchrony: The temporal role of context in team behavior. In E. Mannix, \& M. Neale (Eds.), Research on Managing Groups and Teams, Volume 2: Groups in Context (pp. 33-48). Greenwich, CT: JAI Press Inc.

Ancona, D.G., Goodman, P.S., Lawrence, B.S., \& Tushman, M.L. (2001). Time: A new research lens. Academy of Management Review, 26, 645-663.

Apesteguia, J., Azmat, G., \& Iriberri, N. (2012). The impact of gender composition on team performance and decision making: Evidence from the field. Management Science, 58, 7893.

Arrow, H., \& McGrath, J.E. (1995). Membership dynamics in groups at work: A theoretical framework. In B. M. Staw, \& L. L. Cummings (Eds.). Research in Organizational Behavior (pp.373-411). Greenwich, CT: JAI Press Inc.

Baard, S.K., Rench, T.A., \& Kozlowski, S.W.J. (2014). Performance adaptation: A theoretical integration and review. Journal of Management, 40, 48-99.

Badke-Schaub, P., Goldschmidt, G. and Meijer, M. (2010) How does cognitive conflict in design teams support the development of creative ideas?. Creativity and Innovation Management, 19, 119-133.

Baker, D.P., Day, R., \& Salas, E. (2006). Teamwork as an essential component of highreliability organizations. Health Research and Educational Trust, 41, 1576-1598. 
Balkundi, P., \& Harrison, D.A. (2006). Ties, leaders, and time in teams: Strong inference about network structure's effects on team viability and performance. Academy of Management, 49, 49-68.

Barnes, C.M., Hollenbeck, J.R., Wagner, D.T., DeRue, D.S., Nahrgang, J.D., \& Schwind, K.M. (2008). Harmful help: The costs of backing-up behavior in teams. Journal of Applied Psychology, 93, 529-539.

Bartel, C.A., \& Milliken, F.J. (2004). Perceptions of time in work groups: Do members develop shared cognitions about their temporal demands?. In S. Blount (Ed.). Research on Managing Groups and Teams: Time in Groups (Vol. 6) (pp.87-110). New York: Elsevier.

Bayazit, M., \& Mannix, E. (2003), “Should I stay or should I go? Predicting team members' intent to remain in the team", Small Group Research, 34, 290-321.

Beersma, B., Hollenbeck, J.R., Humphrey, S.E., Moon, H., Conlon, D.E., \& Ilgen, D.R. (2003). Cooperation, competition, and team performance: toward a contingency approach. The Academy of Management Journal, 46, 572-590.

Behfar, K.J., Peterson, R.S., Mannix, E.A., and Trochim, W.M.K. (2008). The critical role of conflict resolution in teams: A close look at the links between conflict type, conflict management strategies, and team outcomes. Journal of Applied Psychology, 93, 170-188.

Bell, S.T., Marentette, B.J. (2011). Team viability for long-term and ongoing organizational teams. Organizational Psychology Review, 1, 275-292.

Bell, S.T., Villado, A.J., Lukasik, M.A., Belau, L., \& Briggs, A.L. (2010). Getting specific about demographic diversity variable and team performance relationships: A meta-analysis. Journal of Management, 37, 709-743.

Biemann, T., Cole, M.S., \& Voelpel, S. (2012). Within-group agreement: On the use (and misuse) of $r_{w g}$ and $r_{w g(j)}$ in leadership research and some best practice guidelines. The Leadership Quarterly, 23, 66-80.

Bledow, R., Frese, M., Anderson, N., Erez, M. \& Farr, J. (2009) A dialectic perspective on innovation: Conflicting demands, multiple pathways, and ambidexterity. Industrial and Organizational Psychology, 2, 305-337.

Bliese, P. D. (2000). Within-group agreement, non-independence, and reliability: Implications for data aggregation and analysis. In K. J. Klein, \& S.W.J. Kozlowski (Eds.), Multilevel Theory, Research, and Methods in Organizations (pp. 349-381). San Francisco: Jossey-Bass.

Bliese, P.D., \& Ployhart, R.E. (2002). Growth modeling using random coefficient models: Model building, testing, and illustrations. Organizational Research Methods, 5, 362-387. 
References

Bluedorn, A.C. \& Jaussi, K.S. (2008). Leaders, followers, and time. The Leadership Quarterly, 19, 654-668.

Bluedorn, A.C., Kalliath, T.J., Strube, M.J. \& Martin, G.D. (1999). Polychronicity and the Inventory of Polychronic Values (IPV): The development of an instrument to measure a fundamental dimension of organizational culture. Journal of Managerial Psychology, 14, 205-230.

Boies, K. \& Howell, J.M. (2006). Leader-member exchange in teams: An examination of the interaction between relationship differentiation and mean LMX in explaining teamlevel outcomes. The Leadership Quarterly, 17, 246-257.

Borgatti, S.P., Everett, M.G., \& Freeman, L.C. (2002). Ucinet for Windows: Software for Social Network Analysis. Harvard, MA: Analytic Technologies.

Borgatti, S., Everett, M.G., \& Freeman, L.C. (1992). UCINET IV network analysis software. Columbia: Analytic Technologies.

Brannick, M. T., Chan, D., Conway, J.M., Lance, C.E., \& Spector, P.E. (2010). What is method variance and how can we cope with it? A panel discussion. Organizational Research Methods, 13, 407-420.

Brewer, W.F. (1987). Schemas versus mental models in human memory. In P. Morris (Ed.), Modelling cognition (pp. 187-197). Chichester, UK: John Wiley \& Sons.

Brewer, W.F. (2003). Mental models. In L. Nadel (Ed.). Encyclopedia of Cognitive Science (Vol. 3, pp. 1-6). London: Macmillan.

Brewer, W.F. (1999). Schemata. In R.A. Wilson \& F.C. Keil (Eds.), The MIT encyclopedia of the cognitive sciences (pp. 729-730). Cambridge, MA: MIT Press.

Bunderson, J.S. \& Sutcliffe, K.M. (2003a). Management team learning orientation and business unit performance. Journal of Applied Psychology, 88, 552-560. doi: 10.1037/00219010.88 .3 .552

Bunderson, J.S. \& Sutcliffe, K.M. (2003b). When to put the brakes on learning. Harvard Business Review, 81, 20-21.

Burke, C.S., Stagl, K.C., Salas, E., Pierce, L., \& Kendal, D. (2006a). Understanding team adaptation: A Conceptual analysis and model. Journal of Applied Psychology, 91, 11891207.

Burke, C.S., Stagl, K.C., Klein, C., Goodwin, G.F., Salas, E., \& Halpin, S.M. (2006b). What type of leadership behaviors is functional in teams? A meta-analysis. The Leadership Quarterly, 17, 288-307. 
Burtscher, M.J., Kolbe, M., Wacker, J., \& Manser, T. (2011). Interactions of team mental models and monitoring behaviors predict team performance in simulated anesthesia inductions. Journal of Experimental Psychology: Applied, 17, 257-269.

Bütikofer, R. (2013). Report on reindustrialising Europe to promote competitiveness and sustainability

(2013/2006(INI)). Retrieved from European Parliament website:

http://www.europarl.europa.eu/sides/getDoc.do?pubRef=-

//EP//NONSGML+REPORT+A7-2013-0464+0+DOC+PDF+V0//EN

Byrne, B.M. (2012). Structural Equation Modeling with Mplus: Basic concepts, applications, and programming. New York: Routledge.

Cannon-Bowers, J.A., Salas, E., \& Converse, S. (1993). Shared mental models in expert team decision-making. In N.J. Castellan Jr. (Ed.), Individual and group decision-making: Current issues (pp. 221-246). Hillsdale, NJ: Lawrence Erlbaum,

Cannon-Bowers, J.A. \& Salas, E. (2001). Reflections on shared cognition. Journal of Organizational Behavior, Vol. 22, pp. 195-202.

Cannon-Bowers, J.A., Tannenbaum, S.I., Salas, E., \& Volpe, C.E. (1995). Defining competencies and establishing team training requirements. In R. Guzzo, \& E. Salas (Eds.), Team effectiveness and decision making in organizations (pp. 333-380). San Francisco, CA: Jossey-Bass.

Carley, K.M. (1997). Extracting team mental models through textual analysis. Journal of Organizational Behavior, 18, 533-558.

Carson, J.B., Tesluk, P.E., \& Marrone, J.A. (2007). Shared leadership in teams: An investigation of antecedent conditions and performance. Academy of Management Journal, 50, 1217-1234.

Casimir, G. (2001). Combinative aspects of leadership style: The ordering and temporal spacing of leadership behaviors. The Leadership Quarterly, 12, 245-278.

Cataldo, M., \& Herbsleb, J.D. (2013). Coordination breakdowns and their impact on development productivity and software failures. IEEE Transactions on Software Engineering, 39, 343-360.

Chan, D. (1998). Functional relations among constructs in the same content domain at different levels of analysis: A typology of composition models. Journal of Applied Psychology, 83, 234-246.

Chen, M-H. (2006). Understanding the benefits and detriments of conflict on team creativity process. Creativity and Innovation Management, 15, 105-116. 
References

Clemens, J.K., \& Dalrymple, S. (2005). Time mastery: How temporal intelligence will make you a stronger, more effective leader. New York, NY: American Management Association.

Cohen, J. (1988), Statistical power analysis for the behavioral sciences, Lawrence Erlbaum Associates, Hillsdale, NJ.

Contractor, N.S. \& Su, C. (2012). Understanding groups from a network perspective. In A. B. Hollingshead, \& M.S. Poole (Eds.), Research methods for studying groups and teams: A guide to approaches, tools, and technologies (pp. 284-310). New York: Routledge.

Cooke, N.J., Gorman, J.C., Myers, C., \& Duran, J. (2012). Theoretical underpinning of interactive team cognition. In E. Salas, S.M. Fiore, \& M.P. Letsky (Eds.), Theories of team cognition: Cross-disciplinary perspectives (pp. 187-207). New York, NY: Routledge.

Cooke, N.J., Kiekel, P.A., \& Helm, E.E. (2001). Measuring team knowledge during skill acquisition of a complex task. International Journal of Cognitive Ergonomics, 5, 297-315.

Costa, P.L., Graça, A.M., Marques-Quinteiro, P., Santos, C.M., Caetano, A., \& Passos, A.M. (2013) Multilevel research in the field of Organizational Behavior: An empirical look at 10 years of theory and research. SAGE Open, 1-17.

Costa, P., Passos, A.M., \& Bakker, A. (2014). Empirical validation of the team work engagement construct. Journal of Personnel Psychology, 13, 34-45.

Coultas, C.W., Driskell, T., Burke, C.S., \& Salas, E. (2014). A conceptual review of emergent state measurement: Current problems, future solutions. Small Group Research, 45, 671703.

Cronin, M.A. \& Weingart, L.R. (2007). Representational gaps, information processing, and conflict in functionally diverse teams. Academy of Management Review, 32, 761-773.

Cronin, M.A., Weingart, L.R. \& Todorova, G. (2011). Dynamics in groups: Are we there yet? The Academy of Management Annals, 5, 571-612.

Culpepper, S.A. \& Aguinis, H. (2011). R is for revolution: A review of a cutting-edge, free, open source statistical package. Organizational Research Methods, 14, 735-740.

Curseu, P.L., Kenis, P. \& Raab, J. (2009). Reciprocated relational preferences and intra-team conflict, Team Performance Management, 15, 18-34.

Dawson, J.F. (2013). Moderation in management research: What, why, when and how. Journal of Business and Psychology, 29, 1-19.

De Dreu, C.K.W. (2006) When too little or too much hurts: Evidence for a curvilinear relationship between task conflict and innovation in teams. Journal of Management, 32, 83-107. 
De Dreu, C.K.W. \& Van Vianen, A.E.M. (2001) Managing relationship conflict and the effectiveness of organizational teams. Journal of Organizational Behavior, 22, 309-328.

De Dreu, C.K.W. \& Weingart, L.R. (2003). Task versus relationship conflict, team performance, and team member satisfaction: A meta-analysis. Journal of Applied Psychology, 88, 741-749.

De Jong, S.B., Van der Vegt, G.S., \& Molleman, E. (2007). The relationships among asymmetry in task dependence, perceived helping behavior, and trust. Journal of Applied Psychology, 92, 1625-1637.

De Vries, R.E., Roe, R.A., \& Taillieu, T.C.B. (2002). Need for Leadership as moderator of the relationship between leadership and individual outcomes. The Leadership Quarterly, 13, $121-137$.

de Wit, F.R.C., Greer, L.L. \& Jehn, K.A. (2012) The paradox of intragroup conflict: A metaanalysis, Journal of Applied Psychology, 97, 360-390.

DeChurch, L.A., \& Mesmer-Magnus, J.R. (2010a). The cognitive underpinnings of effective teamwork: A meta-analysis. Journal of Applied Psychology, 95, 32-53.

DeChurch, L.A., Hiller, N.J., Murase, T., Doty, D., \& Salas, E. (2010). Leadership across levels: Levels of leaders and their levels of impact. The Leadership Quarterly, 21, 10691085.

DeChurch, L.A., Mesmer-Magnus, J. R. \& Doty, D. (2013) Moving beyond relationship and task conflict: Toward a process-state perspective. Journal of Applied Psychology, 98, 559578.

Decuyper, S., Dochy, F., \& Van den Bossche, P. (2010). Grasping the dynamic complexity of team learning: An integrative model for effective team learning in organizations. Educational Research Review, 5, 111-133.

Desivilya, H.S., Somech, A. \& Lidgoster, H. (2010). Innovation and conflict management in work teams: The effects of team identification and task and relationship conflict, Negotiation and Conflict Management Research, 3, 28-48.

Devine, D.J., Clayton, L.D., Phillips, J.L., Dunford, B.B., \& Melner, S.B. (1999). Teams in organizations: Prevalence, characteristics, and effectiveness. Small Group Research, 30, 678-711.

Dijksterhuis, A., van Knippenberg, A., Kruglanski, A.W., \& Schape, C. (1996). Motivated social cognition: Need for closure effects on memory and judgment. Journal of Experimental Social Psychology, 32, 254-270. 
References

DiLiello, T.C., Houghton, J.D. \& Dawley, D. (2011). Narrowing the creativity gap: The moderating effects of perceived support for creativity. Journal of Psychology, 145, 151 172.

Dionne, S.D., Yammarino, F.J., Atwater, L.E., \& James, L.R. (2002). Neutralizing substitutes for leadership theory: Leadership effects and common-source bias. Journal of Applied Psychology, 87, 454-464.

Dionne, S.D., Yammarino, F.J., Howell, J.P., \& Villa, J. (2005). Substitutes for leadership, or not. The Leadership Quarterly, 16, 169-193.

Dlabajová, M. (2015). Report on creating a competitive EU labour market for the 21st century: matching skills and qualifications with demand and job opportunities, as a way to recover from the crisis (2014/2235(INI)). Retrieved from European Parliament website:

http://www.europarl.europa.eu/sides/getDoc.do?pubRef=//EP//NONSGML+REPORT+A8-2015-0222+0+DOC+PDF+V0//EN

Edmondson, A. (1999). Psychological safety and learning behavior in work teams. Administrative Science Quarterly, 44, 350-383.

Edmondson, A.C. (2003). Managing the risk of learning: Psychological safety in work teams. In M. West (Ed.), International Handbook of Organizational Teamwork, (pp. 255-276). London: Blackwell Publishing.

Edmondson, A.C., Dillon, J.R., \& Roloff, K.S. (2007). Chapter 6: Three Perspectives on Team Learning. The Academy of Management Annals, 1, 269-314.

Edmondson, A.C., Winslow, A.B., Bohmer, R.M., \& Pisano, G.P. (2003). Learning how and learning what: Effects of tacit and codified knowledge on performance improvement following technology adoption. Decision Sciences, 34, 197-223.

Edwards, B.D., Day, E.A., Arthur, W., \& Bell, S.T. (2006). Relationships among team ability composition, team mental models, and team performance. Journal of Applied Psychology, 91, 727-736.

Endsley, M.R. (1995). Toward a theory of situation awareness in dynamic systems. Human Factors, 37, 32-64.

Ensley, M.D., Pearson, A.W., \& Amason, A.C. (2002). Understanding the dynamics of new venture top management teams: Cohesion, conflict and new venture performance, Journal of Business Venturing, 17, 365-386. 
Ericksen, J. \& Dyer, L. (2004). Right from the start: Exploring the effects of early team events on subsequent project team development and performance. Administrative Science Quarterly, 49, 438-471.

Ericsson, K A., Krampe, R.T., \& Tesch-Römer, C. (1993). The role of deliberate practice in the acquisition of expert performance. Psychological review, 100, 363-406.

Espinosa, J.A. \& Clark, M. (2014). Team knowledge representation: A network perspective. Human Factors, 56, 333-348.

Espinosa, J.A., Lerch, F.J., \& Kraut, R.E. (2004). Explicit versus implicit coordination mechanisms and task dependencies: One size does not fit all. In E. Salas, \& S.M. Fiore (Eds.). Team cognition: Understanding the factors that drive process and performance (pp. 107129). Washington, DC: American Psychological Association.

Fiol, C.M. \& Lyles, M.A. (1985). Organizational learning. Academy of Management Review, 10, 803-813.

Fishbach, A., Friedman, R.S., \& Kruglanski, A.W. (2003). Leading us not unto temptation: Momentary allurements elicit overriding goal activation. Journal of Personality and Social Psychology, 84, 296-309.

Fiske, S.T. \& Taylor, S.E. (1994). Social cognition. New York: McGraw-Hill.

Fleishman, E.A. (1995). Consideration and structure: Another look at their role in leadership research. In F. Dansereau \& F. J. Yammarino (Eds.), Leadership: The multiple-level approaches (pp. 51-60). Stamford, CT: JAI.

Fussell, S.R. \& Krauss, R.M. (1989). The effects of intended audience on message production and comprehension: Reference in a common ground framework. Journal of Experimental Social Psychology, 25, 203-219.

Gersick, C.J.G. (1988). Time and transition in work teams: Toward a new model of group development. Academy of Management Journal, 31, 9-41.

Gersick, C.J.G., \& Hackman, J.R (1990). Habitual routines in task-performing groups. Organizational Behavior and Human Decision Processes, 47, 65-97.

Gevers, J.M.P. \& Petters, M.A.G. (2009). A pleasure working together? The effects of dissimilarity in team member conscientiousness on team temporal processes and individual satisfaction. Journal of Organizational Behavior, 30, 379-400.

Gevers, J.M.P., Rutte, C.G., \& van Eerde, W. (2006). Meeting deadlines in work groups: Implicit and explicit mechanisms. Applied Psychology: An International Review, 55, 52-72.

Gevers, J.M.P., van Eerde, W., Rutte, C.G. (2009). Team self-regulation and meeting deadlines 
in project teams: Antecedents and effects of temporal consensus. European Journal of Work and Organizational Psychology, 18, 295-321.

Gladstein, D.L. (1984). Groups in context: A model of task group effectiveness. Administrative Science Quarterly, 29, 499-517.

Graen, G.B., \& Uhl-Bien, M. (1995). Relationship-based approach to leadership: Development of Leader-Member Exchange (LMX) theory of leadership over 25 years: Applying a multi-level multi-domain perspective. Leadership Quarterly, 6, 219-247.

Gregory, M.E., Feitosa, J., Driskell, T., Salas, E., \& Vessey, W.B. (2013). Designing, delivering, and evaluating team training in organizations. In E. Salas, S.I. Tannenbaum, D. Cohen, \& G. Latham (Eds.), Developing and enbancing teamwork in organizations: Evidence-based best practices and guidelines (pp. 441-487). San Francisco, CA: Jossey-Bass.

Guchait, P. \& Hamilton, K. (2013). The temporal priority of team learning behaviors vs. shared mental models in service management teams. International Journal of Hospitality Management, 33, 19-28.

Guchait, P. (2016). The mediating effect of team engagement between team cognitions and team outcomes in service-management teams. Journal of Hospitality \& Tourism Research, 40, 139-161

Guenter, H., Van Emmerik, I.J.H., \& Schreurs, B. (2014). The negative effects of delays in information exchange: Looking at workplace relationships from an affective events perspective. Human Resource Management Review, 24, 283-298.

Guiette, A., \& Vandenbempt, K. (2013). Exploring team mental model dynamics during strategic change implementation in professional service organizations. A sensemaking perspective. European Management Journal, 31, 728-744.

Gurtner, A., Tschan, F., Semmer, N.K., \& Nägele, C. (2007). Getting groups to develop good strategies: Effects of reflexivity interventions on team process, team performance, and shared mental models, Organizational Behavior and Human Decision Processes, 102,127-142.

Hackman, J.R. (1987). The design of work teams. In J.W. Lorsch (Ed.). Handbook of organizational behavior (pp. 315-342). New York: Prentice Hall.

Hackman, J.R., \& Wageman, R. (2005). A theory of team coaching. Academy of Management Review, 30, 269-287.

Hackman, J.R. (2012). From causes to conditions in group research. Journal of Organizational Behavior, 33, 428-444. 
Halbesleben, J.R.B., Novicevic, M.M., Harvey, M.G., \& Buckley, R. (2003). Awareness of temporal complexity in leadership of creativity and innovation: A competency-based model. The Leadership Quarterly, 14, 433-454.

Hayes, A.F., Preacher, K.J., \& Myers, T.A. (2011). Mediation and the estimation of indirect effects in political communication research. In E.P. Bucy \& R.L. Holbert (Eds.), The sourcebook for political communication research: Methods, measures, and analytical techniques (pp. 434-465). New York: Routledge.

Hayes, A.F. (2013) An introduction to mediation, moderation, and conditional process analysis: $A$ regression-based approach. New York, NY: Guilford Press.

Hinds, P. \& Mortensen, M. (2005). Understanding conflict in geographically distributed teams: An empirical investigation. Organization Science, 16, 290-307.

Hinsz, V.B., Tindale, R.S., \& Vollrath, D.A. (1997). The emerging conceptualization of groups as information processors. Psychological Bulletin, 121, 43-64.

Hoffman, R.R. \& Deffenbacher, K.A. (1992). A brief history of applied cognitive psychology. Applied Cognitive Psychology, 6, 1-48 .

Holland, J.H., Holyoak, K.J., Nisbett, R.E., \& Thagard P.R. (1986). Induction: processes of inference, learning, and discovery. Massachusetts, USA: MIT Press, Cambridge.

Hox, J.J. (2010). Multilevel analysis: Techniques and applications. $2^{\text {nd }}$ edition. New York: Routledge.

Hox, J.J. (2012, March). Statistical Modeling in Mplus. 41th GESIS Spring Seminar: Topics in Social Science Data Analysis: Causality, Structural Equation Modeling with AMOS and Mplus. GESIS - Leibniz-Institute for the Social Sciences.

Hu, L. \& Bentler, P.M. (1999). Cut of criteria for fit indexes in covariance structure analysis: Conventional criteria versus new alternatives. Structural Equation Modeling, 6, 1-55.

Hülsheger, U.R., Anderson, N., \& Salgado J.F. (2009) Team-level predictors of innovation at work: A comprehensive meta-analysis spanning three decades of research. Journal of Applied Psychology, 94, 1128-1145.

Humphrey, S.E., Morgeson, F.P., \& Mannor, M.J. (2009). Developing a theory of the strategic core of teams: A role composition model of team performance. Journal of Applied Psychology, 94, 48-61.

Iacobucci, D., Posavac, S.S., Kardes, F.R., Schneider, M.J., \& Popovich, D.L. (2015a). Toward a more nuanced understanding of the statistical properties of a median split. Journal of Consumer Psychology, 25, 652-665. 
References

Iacobucci, D., Posavac, S.S., Kardes, F.R., Schneider, M.J., \& Popovich, D.L. (2015b). The median split: Robust, refined, and revived. Journal of Consumer Psychology, 25, 690-704.

Ilgen, D.R., Major, D.A., Hollenbeck, J.R., \& Sego, D.J. (1995). Raising an individual decisionmaking model to the team level: A new research model and paradigm. In R. A. Guzzo, \& E. Salas (Eds.), Team effectiveness and decision making in organizations (pp.113148). San Francisco: Jossey-Bass.

Ilgen, D.R., Hollenbeck, J.R., Johnson, M. \& Jundt, D. (2005). Teams in organizations: From input-process-output models to IMOI models. Annual Review of Psychology, 56, 517-543.

James, L.R., Demaree, R.J., \& Wolf, G. (1993). $r_{w g}$ : An assessment of within group interrater agreement. Journal of Applied Psychology, 78, 306-309.

Janicik, G.A., \& Bartel, C.A. (2003). Talking about time: Effects of temporal planning and time awareness norms on group coordination and performance. Group Dynamics: Theory, Research, and Practice, 7, 122-134.

Janis, I. (1982). Groupthink (2nd ed.). Boston: Houghton-Mifflin.

Janis, I.L. (1972). Victims of groupthink: A psychological study of foreign-policy decisions and fiascoes. Boston, USA: Houghton-Mifflin.

Jansen, K.J. \& Kristof-Brown, A.L. (2005). Marching to the beat of a different drummer: Examining the impact of pacing congruence. Organizational Behavior and Human Decision Processes, 97, 93-105.

Jehn, K.A. \& Mannix, E. (2001). The dynamic nature of conflict: A longitudinal study of intragroup conflict and group performance. Academy of Management Journal, 44, 238-251.

Jehn, K.A. (1995) A multi method examination of the benefits and detriments of intragroup conflict. Administrative Science Quarterly, 40, 256-282.

Jehn, K.A. (1997). A qualitative analysis of conflict types and dimensions in organizational groups. Administrative Science Quarterly, 42, 530-557.

Johnson, T.E., Lee, Y., Lee, M., O'Connor, D.L., Khalil, M.K. \& Huang, X. (2007) Measuring sharedness of team-related knowledge: Design and validation of a shared mental model instrument. Human Resource Development International, 10, 437-454.

Johnson-Laird, P. (1983). Mental models. Cambridge, MA: Harvard University Press.

Jones, N.A., Ross, H., Lynam, T., Perez, P. \& Leitch A. (2011). Mental models: an interdisciplinary synthesis of theory and methods. Ecology and Society, 16.

Kameda, T., Tindale, R.S., \& Davis, J.H. (2003). Cognitions, preferences, and social sharedness: Past, present, and future directions in group decision making. In S.L. 
Schneider \& J. Shanteau (Eds.), Emerging perspectives on judgment and decision research (pp.215-240). Cambridge, UK: Cambridge University Press.

Kanfer, R., \& Ackerman, P.L. (1989). Motivation and cognitive abilities: An integrative/ aptitude-treatment interaction approach to skill acquisition. Journal of Applied Psychology, 74, 657-690.

Kerr, S. \& Jermier, J.M. (1978). Substitutes for leadership: Their meaning and measurement. Organizational Behavior and Human Performance, 22, 375-403.

Klimoski, R., \& Mohammed, S. (1994). Team mental model: Construct or metaphor? Journal of Management, 20, 403-437.

Kozlowski, S.W.J. \& Bell, B.S. (2003). Work groups and teams in organizations. In W.C. Borman, D.R. Ilgen \& R.J. Klimoski (Eds.), Handbook of psychology (Vol. 12): Industrial and Organizational Psychology (333-375). New York: Wiley-Blackwell.

Kozlowski, S.W.J., \& Bell, B.S. (2008). Team learning, development, and adaptation. In V.I. Sessa, \& M. London (Eds.), Work, group learning: Understanding, improving and assessing how groups learn in organizations (pp.15-44). New York: Taylor \& Francis Group.

Kozlowski, S.W.J., \& Ilgen, D.R. (2006). Enhancing the effectiveness of work groups and teams. Psychological Science in the Public Interest, 7, 77-124.

Kozlowski, S.W.J., \& Klein, K.J. (2000). A multi-level approach to theory and research in organizations: Contextual, temporal, and emergent processes. In K. J. Klein \& S. W. J. Kozlowski (Eds.), Multilevel theory, research, and methods in organizations. (pp.3-90). San Francisco: Jossey-Bass.

Kozlowski, S.W.J., Gully, S.M., Nason, E.R., \& Smith, E.M. (1999). Developing adaptive teams: A theory of compilation and performance across levels and time. In D.R. Ilgen \& E.D. Pulakos (Eds.), The changing nature of work performance: Implications for staffing, personnel actions, and development (pp. 240-292). San Francisco, CA: Jossey-Bass.

Kozlowski, S.W.J., Toney, R.J., Mullins, M.E., Weissbein, D.A., Brown, K.G., \& Bell, B.S. (2001). Developing adaptability: A theory for the design of integrated-embedded training systems. In E. Salas (Ed.), Advances in Human Performance and Cognitive Engineering Research (pp. 59-123). New York: JAI Press.

Kozlowski, S.W.J., \& Chao, G.T. (2012a). The dynamics of emergence: Cognition and cohesion in work teams. Managerial and Decision Economics, 33, 335-354.

Kozlowski, S.W.J., \& Chao, G.T. (2012b). Macrocognition, team learning, and team knowledge: Origins, emergence, and measurement. In E. Salas, S.M. Fiore, \& M. P. 
References

Letsky (Eds.), Theories of team cognition: Cross-disciplinary perspectives (pp. 19-48). New York: Routledge.

Kozlowski, S.W.J., Chao, G.T. \& Jensen, J.M. (2010). Building an infrastructure for organizational learning: A multilevel approach. In S.W.J. Kozlowski \& E. Salas (Eds.), Learning training, and development in organizations (pp. 363-403). New York: Routledge.

Kozlowski, S.W.J., Watola, D.J., Jensen, J.M., Kim, B. H., \& Botero, I.C. (2009). Developing adaptive teams: A theory of dynamic team leadership. In E. Salas, G.F. Goodwin, \& C.S. Burke (Eds.), Team effectiveness in complex organizations: Cross-disciplinary perspective and approaches (pp. 113-155). New York: Routledge, Taylor \& Francis Group.

Kraiger, K., Ford, J. K., \& Salas, E. (1993). Application of cognitive, skill-based, and affective theories of learning outcomes to new methods of training evaluation. Journal of Applied Psychology, 78, 311-328.

Krauss, R.M. \& Fussell, S.R. (1991). Constructing shared communicative environments. In L.B. Resnick, J.M. Levine \& S.D. Teasley (Eds.). Perspectives on Socially-shared Cognition. Washington, D.C.: American Psychological Association.

Kulik, J.A., \& Kulik, C.L.C. (1988). Timing of feedback and verbal learning. Review of Educational Research, 58, 79-97.

Künzle, B., Zala-Mezö, E., Kolbe, M., Wacker, J., \& Grote, G. (2010). Substitutes for leadership in anaesthesia teams and their impact on leadership effectiveness. European Journal of Work and Organizational Psychology, 19, 505-531.

Lance, C.E., Dawson, B., Birkelbach, D. \& Hoffman, B.J. (2010). Method effects, measurement error, and substantive conclusions. Organizational Research Methods, 13, $435-455$.

Lau, R.S. \& Cobb, A.T. (2010). Understanding the connections between relationship conflict and performance: The intervening roles of trust and exchange, Journal of Organizational Behavior, 31, 898-917.

LePine, J.A., Hollenbeck, J.R., Ilgen, D.R., Colquitt, J.A., \& Ellis, A. (2002). Gender composition, situational strength, and team decision-making accuracy: A criterion decomposition approach. Organizational Behavior and Human Decision Processes, 88, 445475 .

LePine, J.A., Piccolo, R.F., Jackson, C.L., Mathieu, J.E., \& Saul, J.R. (2008). A meta-analysis of teamwork processes: Tests of a multidimensional model and relationships with team effectiveness criteria, Personnel Psychology, 61, 273-308. 
Levesque, L.L., Wilson, J.M., \& Wholey, D.R. (2001). Cognitive divergence and shared mental models in software development project teams, Journal of Organizational Behavior, 22, 135-144.

Levine, J.M. \& Resnick, L. B. (1993). Social foundations of cognition. Annual Review of Psychology, 44, 585-612.

Lewis, K. (2003). Measuring transactive memory systems in the field: Scale development and validation. Journal of Applied Psychology, 88, 587-604.

Lim, B-C., \& Klein, K.J. (2006). Team mental models and team performance: A field study of the effects of team mental model similarity and accuracy. Journal of Organizational Behavior, 27, 403-418.

Liu, S., Hu, J., Li, Y., Wang, Z., \& Lin, X. (2014). Examining the cross-level relationship between shared leadership and learning in teams: Evidence from China. The Leadership Quarterly, 25, $282-295$.

Lorinkova, N.M., Pearsall, M.J., \& Sims Jr., H.P. (2013). Examining the differential longitudinal performance of directive versus empowering leadership in teams. Academy of Management Journal, 56, 573-596.

MacMillan, J., Entin, E.E., \& Serfaty, D. (2004). Communication overhead: The hidden cost of team cognition. In E. Salas \& S. M. Fiore (Eds.), Team cognition: Understanding the factors that drive process and performance (pp. 61-82). Washington, DC: American Psychological Association.

March, J.G. (1991). Exploration and exploitation in organizational learning. Organization Science, 2, 71-87.

Marks, M.A., Mathieu, J.E., \& Zaccaro, S.J. (2001). A temporally based framework and taxonomy of team processes, Academy of Management Review, 26, 356-376.

Marks, M.A., Zaccaro, S.J., \& Mathieu, J.E. (2000). Performance implications of leader briefings and team-interaction training for team adaptation to novel environments. Journal of Applied Psychology, 85, 971-986.

Marks, M.A., Sabella, M.J., Burke, C.S., \& Zaccaro, S.J. (2002). The impact of cross-training on team effectiveness, Journal of Applied Psychology, 87, 3-13.

Marques-Quinteiro, P., Curral, L., Passos, A.M., \& Lewis, K. (2013). And now what do we do? The role of transactive memory systems and task coordination in action teams. Group Dynamics: Theory, Research, and Practice, 17, 194-206. 
References

Marques-Quinteiro, P., Passos, A.M., Curral, L. (2014). Thought self-leadership and effectiveness in self-management teams. Leadership. Advance online publication.

Maruping, L.M., Venkatesh, V., Thatcher, S.M.B., \& Patel, P.C. (2015). Folding under pressure or rising to the occasion? Perceived time pressure and the moderating role of team temporal leadership. Academy of Management Journal, 58, 1313-1333.

Massaro, D.W., \& Cowan, N. (1993). Information processing models: Microscopes of the mind. Annual Review of Psychology, 44, 383-425.

Mathieu, J.E. \& Rapp, T.L. (2009). Laying the foundation for successful team performance trajectories: The roles of team charters and performance strategies, Journal of Applied Psychology, 94, 90-103.

Mathieu, J.E., \& Taylor, S.R. (2006). Clarifying conditions and decision points for mediational type inferences in Organizational Behavior. Journal of Organizational Behavior, 27, 10311056.

Mathieu, J.E., DeShon, R.P., \& Bergh, D.D. (2008a). Mediational inferences in organizational research: Then, now, and beyond. Organizational Research Methods, 11, 203-223.

Mathieu, J.E., Heffner, T.S., Goodwin, G.F., Salas, E., \& Cannon-Bowers, J.A. (2000). The influence of shared mental models on team process and performance. Journal of Applied Psychology, 85, 273-283.

Mathieu, J.E., \& Rapp, T.L. (2009). Laying the foundation for successful team performance trajectories: The roles of team charters and performance strategies. Journal of Applied Psychology, 94, 90-103.

Mathieu, J.E., \& Taylor, S.R. (2006). Clarifying conditions and decision points for mediational type inferences in Organizational Behavior. Journal of Organizational Behavior, 27, 10311056.

Mathieu, J.E., Heffner, T.S., Goodwin, G.F., Cannon-Bowers, J.A., \& Salas, E. (2005). Scaling the quality of teammates' mental models: Equifinality and normative comparisons, Journal of Organizational Behavior, 26, 37-56.

Mathieu, J.E., Maynard, M.T., Rapp, T. \& Gilson, L. (2008b). Team effectiveness 1997-2007: A review of recent advancements and a glimpse into the future. Journal of Management, 34, 410-476.

Mathieu, J.E., Rapp, T.L., Maynard, M.T., \& Mangos, P.M. (2010). Interactive effects of team and task shared mental models as related to air traffic controllers' collective efficacy and effectiveness, Human Performance, 23, 22-40. 
Maynard, M.T., \& Gilson, L.L. (2014). The role of shared mental model development in understanding virtual team effectiveness. Group \& Organization Management, 39, 3-32.

Maynard, T., Kennedy, D.M., \& Sommer, S.A. (2015). Team adaptation: A fifteen-year synthesis (1998-2013) and framework for how this literature needs to "adapt" going forward. European Journal of Work and Organizational Psychology, 24, 652-677.

McClelland, G.H., Lynch, J.G., Irwin, J.R., Spiller, S.A., \& Fitzsimons, G.J. (2015). Median splits, Type II errors, and false-positive consumer psychology: Don't fight the power. Journal of Consumer Psychology, 25, 679-689.

McGrath, J.E., Arrow, H. \& Berdahl, J.L. (2000). The study of groups: Past, present, and future, Personality and Social Psychology Review, 4, 95-105.

McGrath, J.E. (1964). Social psychology: A brief introduction. New York: Holt, Rinehart and Winston.

McIntyre, R.M., \& Salas, E. (1995). Measuring and managing for team performance: Emerging principles from complex environments. In R.A. Guzzo, \& E. Salas (Eds.), Team effectiveness and decision making in organizations (pp. 9-45). San Francisco, CA: Jossey Bass.

Minionis, D., Zaccaro, S., \& Perez, R. (1995). Shared mental models, team coordination, and team performance. American Journal of Political Science, 45, 259-276.

Mitchell, T.R., \& James, L.R. (2001). Building better theory: Time and the specification of when things happen. Academy of Management Review, 26, 530-547.

Mohammed, S. \& Alipour, K.K. (2014). It's time for temporal leadership: Individual, dyadic, team, and organizational effects. Industrial and Organizational Psychology, 7, 178-203.

Mohammed, S., \& Hamilton, K. (2012). Studying team cognition: The good, the bad, and the practical. In A. B. Hollingshead, \& M.S. Poole (Eds.), Research methods for studying groups and teams: A guide to approaches, tools, and technologies (pp. 132-153). New York: Routledge.

Mohammed, S., \& Nadkarni, S. (2011). Temporal diversity and team performance: The moderating role of team temporal leadership. Academy of Management Journal, 54, 489508.

Mohammed, S., \& Nadkarni, S. (2014). Are we all on the same temporal page? The moderating effects of temporal team cognition on the polychronicity diversity-team performance relationship. Journal of Applied Psychology, 99, 404-22.

Mohammed, S., Ferzandi, L., \& Hamilton, K. (2010). Metaphor no more: A 15-year review of the team mental model construct. Journal of Management, 36, 876-910. 
References

Mohammed, S., Hamilton, K., \& Lim, A. (2009). The incorporation of time in team research: Past, current, and future. In E. Salas, G.F. Goodwin, \& C.S. Burke (Eds.), Team effectiveness in complex organizations: Cross-disciplinary perspective and approaches (pp. 321-348). New York: Routledge, Taylor \& Francis Group.

Mohammed, S., Hamilton, K., Tesler, R., Mancuso, V., \& McNeese, M. (2015). Time for temporal team mental models: Expanding beyond "what" and "how" to incorporate "when”. European Journal of Work and Organizational Psychology, 24, 693-709.

Mohammed, S., Klimoski, R., \& Rentsch, J. R. (2000). The measurement of team mental models: We have no shared schema. Organizational Research Methods, 3, 123-165.

Mohammed, S., Tesler, R., \& Hamilton, K. (2012). Time and shared cognition: Towards greater integration of temporal dynamics. In E. Salas, S.M. Fiore, \& M.P. Letsky (Eds.), Theories of team cognition: Cross disciplinary perspectives (pp. 87 - 116). New York: Routledge, Taylor and Francis Group.

Monteil, J.-M. \& Huguet, P. (1999). Social context and cognitive performance: Towards a social psychology of cognition. United Kingdom: Psychology Press.

Montoya-Weiss, M.M., Massey, A.P., \& Song, M. (2001). Getting it together: Temporal coordination and conflict management in global virtual teams. Academy of Management Journal, 44, 1251-62.

Moreland, R.L., \& McMinn, J. G. (2010). Group reflexivity and performance. In S. R. Thye, E. J. Lawler (Eds.), Advances in Group Processes, Volume 27 (pp. 63-95). Emerald Group Publishing Limited.

Moscovici, S. (1989). Preconditions for explanation in social psychology. European Journal of Social Psychology, 19, 407-430.

Moynihan, D. P. (2007). From forest fires to hurricane Katrina: Case studies of incident command systems. Washington, DC: IBM Center for the Business of Government.

Murase, T., Carter, D.R., DeChurch, L.A., \& Marks, M.A. (2014). Mind the gap: The role of leadership in multiteam system collective cognition. The Leadership Quarterly, 25, $972-$ 986.

Muthén, L.K. \& Muthén, B.O. (2012). Mplus User's Guide. Seventh Edition. Los Angeles, CA: Muthén \& Muthén.

Muthén, L.K. and Muthén, B.O. (1998-2010). Mplus User's Guide, 6 ${ }^{\text {th }}$ edn, Muthén \& Muthén, Los Angeles, CA. 
Nübold, A., Muck, P.M., \& Maier, G.W. (2013). A new substitute for leadership? Followers' state core self-evaluations. The Leadership Quarterly, 24, 29-44.

O’Leary, M.B., Mortensen, M., \& Woolley, A.W. (2011). Multiple team membership: A theoretical model of its effects on productivity and learning for individuals and teams. Academy of Management Review, 36, 461-478.

Orasanu, J. \& Salas, E. (1993). Team decision making in complex environments. In G.A. Klein, J. Orasanu, R. Calderwood, \& C.E. Zsambok (Eds.), Decision making in action: Models and methods (pp. 327-345). Norwood, NJ: Ablex.

Passos, A.M., \& Caetano, A. (2005). Exploring the effects of intragroup conflict and past performance feedback on team effectiveness. Journal of Managerial Psychology, 20, 231244.

Pazos, P. (2012). Conflict management and effectiveness in virtual teams. Team Performance Management, 18, $401-417$.

Pearce, C.L., \& Sims Jr., H.P. (2002). Vertical versus shared leadership as predictors of the effectiveness of change management teams: An examination of aversive, directive, transactional, transformational, and empowering leader behaviors. Group Dynamics: Theory, Research, and Practice, 6, 172-197

Pearsall, M. J., Ellis A. P. J. \& Bell, B. S. (2010). Building the infrastructure: The effects of role identification behaviors on team cognition development and performance. Journal of Applied Psychology, 95, 192-200.

Pearsall, M. J., Ellis A.P.J., \& Bell, B.S. (2010). Building the infrastructure: The effects of role identification behaviors on team cognition development and performance. Journal of Applied Psychology, 95,192-200.

Pelled, L.H., Eisenhardt, K.M., \& Xin, K.R. (1999) Exploring the black box: An analysis of work group diversity, conflict, and performance. Administrative Science Quarterly, 44, 128.

Pinheiro, J.C. \& Bates, D.M. (2000). Mixed-Effects Models in S and S-PLUS. New York: Springer.

Pisano, G.P., Bohmer, R.M., \& Edmondson, A.C. (2001). Organizational differences in rates of learning: Evidence from the adoption of minimally invasive cardiac surgery. Management Science, 47, 752-768.

Pitariu, A.H. \& Ployhart, R.E. (2010). Explaining change: Theorizing and testing mediated longitudinal relationships. Journal of Management, 36, 405-429. 
References

Ployhart, R.E., \& Vandenberg, R.J. (2010). Longitudinal research: The theory, design, and analysis of change. Journal of Management, 36, 94-120.

Podsakoff, P.M., MacKenzie, S.B., \& Bommer, W.H. (1996). Meta-analysis of the relationships between Kerr and Jermier's substitutes for leadership and employee job attitudes, role perceptions, and performance. Journal of Applied Psychology, 81, 380-399.

Preacher, K.J., \& Hayes, A.F. (2008). Asymptotic and resampling strategies for assessing and comparing indirect effects in multiple mediator models. Behavior Research Methods, 40, 879-891.

Pulakos, E.D., Schmitt, N., Dorsey, D.W., Arad, S., Hedge, J.W., \& Borman, W.C. (2002). Predicting adaptive performance: Further tests of a model of adaptability. Human Performance, 15, 299-323.

Randall, K.R., Resick, C.J., \& DeChurch, L.A. (2011). Building team adaptive capacity: The roles of sensegiving and team composition. Journal of Applied Psychology, 96, 525-540.

Rankin, G. \& Stokes, M. (1998). Reliability of assessment tools in rehabilitation: An illustration of appropriate statistical analyses. Clinical Rebabilitation, 12, 187-99.

Rau, D. (2005). The influence of relationship conflict and trust on the transactive memory: Performance relation in top management teams. Small Group Research, 36, 746-771.

Reagans, R., Argote, L., \& Brooks, D. (2005). Individual experience and experience working together: Predicting learning rates from knowing who knows what and knowing how to work together. Management Science, 51, 869-881.

Reed, S.K. (2000). Cognition: Theory and applications ( $5^{\text {th }}$ ed). Belmont, CA: Wadsworth.

Rentsch, J.R. \& Klimoski, R.J. (2001). Why do "great minds" think alike? Antecedents of team member schema agreement. Journal of Organizational Behavior, 22, 107-120.

Rentsch, J.R., \& Mot, I.R. (2012). Elaborating cognitions in teams: Cognitive similarity configurations. In E. Salas, S.M.Fiore \& M.P. Letsky (Eds.), Theories of team cognition: Cross-disciplinary perspectives (pp. 145-170). New York: Routledge.

Rentsch, J.R., Delise, L.A., Salas, E., \& Letsky, M.P. (2010). Facilitating Knowledge Building in Teams: Can a New Team Training. Small Group Research, 41, 505-523.

Resick, C.J., Dickson, M.W., Mitchelson, J.K., Allison, L.K., \& Clark, M.A. (2010a). Team composition, cognition, and effectiveness: Examining mental model similarity and accuracy. Group Dynamics: Theory, Research, and Practice, 14, 174-191. 
Resick, C.J., Murase, T., Bedwell, W. L., Sanz, E., Jiménez, M., \& DeChurch, L.A. (2010b). Mental model metrics and team adaptability: A multi-facet multi-method examination. Group Dynamics: Theory, Research, and Practice, 14, 332-349.

Resnick, L.B. (1993). Shared cognition: Thinking as social practice. In L. B. Resnick, J. M. Levine, \& S. D. Teasley (Eds.), Perspectives on socially shared cognition (pp. 1-20). Washington DC: American Psychological Association.

Rico, R., Sánchez-Manzanares, M., Antino, M., \& Lau, D. (2012). Bridging team faultlines by combining task role assignment and goal structure strategies. Journal of Applied Psychology, 97, 407-420.

Rico, R., Sánchez-Manzanares, M., Gil, F., \& Gibson, C. (2008). Team implicit coordination processes: A team knowledge-based approach. Academy of Management Review, 33, 16384.

Roe, R.A. (2008). Time in applied psychology: The study of "what happens" rather than "what is". The European Psychologist, 13, 37-52.

Roe, R.A. (2009). Perspectives on time and the chronometric study of what happens in organizations. In R.A. Roe, M.J. Waller, \& S.R. Clegg (Eds.). Time in organizational research (pp. 291-313). London: Routledge.

Roe, R.A., Gockel, C. \& Meyer, B. (2012). Time and change in teams: Where we are and where we are moving, European Journal of Work and Organizational Psychology, 21, 629-656.

Rosen, M.A., Bedwell, W.L., Wildman, J.L., Fritzche, B.A., Salas, E., \& Burke, C.S. (2011). Managing adaptive performance in teams: Guiding principles and behavioral markers for measurement. Human Resource Management Review, 21, 107-122.

Rouse, W.B. \& Morris, N.M. (1986). On looking into the black box: Prospects and limits in the search for mental models. Psychological Bulletin, 100, 349-363.

Rucker, D.D., McShane, B.B., \& Preacher, K.J. (2015). A researcher's guide to regression, discretization, and median splits of continuous variables. Journal of Consumer Psychology, 25, 666-678.

Rumelhart, D.E. \& Ortony, A. (1977). The representation of knowledge in memory. In R. C. Anderson, R. J. Spiro \& W. E. Montague (Eds.), Schooling and the acquisition of knowledge (pp. 99 -135). Hillsdale. NJ: Erlbaum

Runco, M.A. \& Jaeger, G.J. (2012). The standard definition of creativity. Creativity Research Journal, 24, 92-96. 
References

Rutherford, A., \& J.R. Wilson (2004). Models of mental models: an ergonomist-psychologist dialogue. In N. Moray (Ed.), Ergonomics major writings: psychological mechanisms and models in ergonomics (pp. 309-323). London, UK: Taylor and Francis.

Salas, E., \& Wildman, J.L. (2009). Ten critical research questions: The need for new and deeper explorations. In E. Salas, G.F. Goodwin, \& C.S. Burke (Eds.), Team effectiveness in complex organizations: Cross-disciplinary perspective and approaches (pp. 525-546). New York: Routledge, Taylor \& Francis Group.

Salas, E. Fiore, S.M. \& Letsky, M.P. (2012). Why cross-disciplinary theories of team cognition?. In E. Salas, S.M. Fiore, \& M.P. Letsky (Eds.), Theories of team cognition: Crossdisciplinary perspectives (pp. 3-18). New York, NY: Routledge.

Salas, E., \& Fiore, S.M. (2004). Why team cognition? An overview. In E. Salas, \& S.M. Fiore (Eds.), Team cognition: Understanding the factors that drive process and performance (pp.3-8). Washington DC: American Psychological Association.

Salas, E., Cooke, N.J. \& Rosen, M.A. (2008). On teams, teamwork, and team performance: Discoveries and developments. Human Factors, 50, 540-547.

Salas, E., Dickinson, T., Converse, S., \& Tannenbaum, S. (1992). Toward an understanding of team performance and training. In R. Swezey \& E. Salas (Eds.), Teams: Their training and performance. Norwood, NJ: Ablex Publishing.

Salas, E., Rosen, M.A., Burke, C.S., \& Goodwin, G.F. (2009). The wisdom of collectives in organizations: An update of the teamwork competencies. In E. Salas, G.F. Goodwin, \& C.S. Burke (Eds.), Team effectiveness in complex organizations: Cross-disciplinary perspectives and approaches (pp. 39-79). New York: Routledge, Taylor \& Francis Group.

Salas, E., Sims, D.E. \& Burke, C.S. (2005). Is there a "Big Five" in teamwork? Small Group Research, 36, 555-599.

Salas, E., Stagl, K.C., Burke, C.S., \& Goodwin, G.F. (2007). Fostering team effectiveness in organizations: Toward an integrative theoretical framework of team performance. In J.W. Shuart, W. Spaulding, \& J. Poland (Eds.), Modelling complex systems: Motivation, cognition and social processes, Nebraska Symposium on Motivation, 52 (pp. 185-243). Lincoln NE: University of Nebraska Press.

Samuelson, C.D., \& Allison, S.T. (1994). Cognitive factors affecting the use of social decision heuristics in resource-sharing tasks. Organizational Behavior and Human Decision Processes, $58,1-27$.

Santos, C.M, \& Passos, A.M. (2013). Team mental models, relationship conflict and 
effectiveness over time. Team Performance Management, 19, 363-385.

Santos, C.M., Uitdewilligen, S., \& Passos, A.M. (2015a). A temporal common ground for learning: The moderating effect of shared mental models on the relation between team learning behaviours and performance improvement. European Journal of Work and Organizational Psychology, 24, 710-725.

Santos, C.M., Uitdewilligen, S., \& Passos, A.M. (2015b). Why your team is more creative than mine? - The influence of shared mental models on intragroup conflict, team creativity and effectiveness. Creativity and Innovation Management, 24, 645-658.

Savelsbergh, C.M.J.H., van der Heijden, B.I.J.M., \& Poel, R.F. (2009). The development and empirical validation of a multidimensional measurement instrument for team learning behaviors. Small Group Research, 40, 578-607.

Savelsbergh, C., Gevers, J., van der Heijden, B.I.J.M., \& Poell, R.F. (2012). Team role stress: Relationships with team learning and performance in project teams. Group \& Organization Management, 37, 67-100.

Schippers, M.C., Homan, A.C., \& van Knippenberg, D. (2013). To reflect or not to reflect: Prior team performance as a boundary condition of the effects of reflexivity on learning and final team performance. Journal of Organizational Behavior, 34, 6-23. doi: 10.1002/job.1784

Schippers, M.C., Den Hartog, D.N., Koopman, P.L., \& van Knippenberg, D. (2008). The role of transformational leadership in enhancing team reflexivity. Human Relations, 61, 15931616. doi: $10.1177 / 0018726708096639$

Schippers, M.C., den Hartog, D.N. \& Koopman, P.L. (2007). Reflexivity in teams: A measure and correlates. Applied Psychology: An International Review, 56, 189-211.

Schippers, M.C., den Hartog, D.N., Koopman, P.L., Wienk, J.A. (2003). Diversity and team outcomes: The moderating effects of outcome interdependence and group longevity and the mediating effect of reflexivity. Journal of Organizational Behavior, 24, 779-802.

Schmidt, R.C., Bienvenu, M., Fitzpatrick, P.A., \& Amazeen, P.G. (1998). A comparison of intra- and interpersonal interlimb coordination: Coordination breakdowns and coupling strength. Journal of Experimental Psychology: Human Perception and Performance, 24, 884-900.

Schreiber, J.B., Stage, F.K., King, J., Nora, A., \& Barlow, E.A. (2006). Reporting structural equation modeling and confirmatory factor analysis results: A review. The Journal of Educational Research, 99, 323-337. 
References

Shah, P.R. \& Jehn, K.A. (1993). Do friends perform better than acquaintances? The interaction of friendship, conflict, and task. Group Decision and Negotiation, 2, 149-166.

Shamir, B. (2011). Leadership takes time: Some implications of (not) taking time seriously in leadership research. The Leadership Quarterly, 22, 307-315.

Shea, G.P., \& Guzzo, R.A. (1987). Groups as human resources. In K.M. Rowland \& G.R. Ferris (Eds.), Research in personnel and human resource management (Vol. 5, pp. 323-356). Greenwich, CT: JAI Press.

Siemsen, E., Roth, A. \& Oliveira, P. (2010). Common method bias in regression models with linear, quadratic, and interaction effects. Organizational Research Methods, 13, 456-476.

Simões, E. (2001). O processo de tomada de decisão. In J.M.C. Ferreira, J. Neves, \& A. Caetano (Eds.). Manual de psicossociologia das organizações (pp. 405-427). Lisboa: McGrawHill.

Simons, T. \& Peterson, R. (2000). Task conflict and relationship conflict in top management teams: The pivotal role of intragroup trust. Journal of Applied Psychology, 85, 102-111.

Singer, J.D., \& Willet, J.B. (2003). Applied longitudinal data analysis: Modeling change and event occurrence. New York: Oxford University Press

Skilton, P.F. \& Dooley, K.J. (2010). The effects of repeat collaboration on creative abrasion. Academy of Management Review, 35, 118-134.

Slaughter, A.J., Yu, J., \& Koehly, L.M. (2009). Social network analysis: Understanding the role of context in small groups and organizations. In E. Salas, G.F. Goodwin, \& C.S. Burke (Eds.), Team effectiveness in complex organizations: Cross-disciplinary perspectives and approaches (pp. 433-459). New York: Routledge, Taylor \& Francis Group.

Sonnentag, S. (2012). Time in organizational research: Catching up on a long neglected topic in order to improve theory. Organizational Psychology Review, 2, 361-368.

Spector, P.E. (1997). Job satisfaction: Application, assessment, causes, and consequences, Sage: Thousand Oaks CA.

Spector, P.E. (2006). Method variance in organizational research: Truth or urban legend? Organizational Research Methods, 9, 221-232.

Stachowski, A.A., Kaplan, S.A., \& Waller, M.J. (2009). The benefits of flexible team interaction during crises. Journal of Applied Psychology, 94, 1536-1543.

Standifer, R.L., Raes, A.M.L., Peus, C., Passos, A.M., Santos, C.M., \& Weisweiler, S. (2015). Time in teams: Cognitions, conflict and team satisfaction. Journal of Managerial Psychology, 30, 692-708. 
Standifer, R., \& Bluedorn, A. (2006). Alliance management teams and entrainment: Sharing temporal mental models. Human Relations, 59, 903-927.

Stout, R.J., Cannon-Bowers, J.A., Salas, E., \& Milanovich, D.M. (1999). Planning, shared mental models, and coordinated performance: An empirical link is established. Human Factors, 41, 61-71.

Sundstrom, E., De Meuse, K.P., \& Futrell, D. (1990). Work teams, applications and effectiveness. American Psychologist, 45, 120-133.

Tabernero, C., Chambel, M.J., Curral, L. \& Arana, J.M. (2009). The role of task-oriented versus relationship-oriented leadership on normative contract and group performance. Social Behavior and Personality, 37, 1391-1404.

Tannenbaum, S.I., Beard, R.L., \& Cerasoli, C.P. (2013). Conducting team debriefings that work: Lessons from research and practice. In E. Salas, S.I. Tannenbaum, D. Cohen, \& G. Latham (Eds.), Developing and enbancing teamwork in organizations: Evidence-based best practices and guidelines (pp. 488-519). San Francisco, CA: Jossey-Bass.

Tasa, K., Taggars, S., \& Seijts, G.H. (2007). The development of collective efficacy in teams: a multilevel and longitudinal perspective. Journal of Applied Psychology, 92, 17-27.

Thompson, L. L. (2004). Making the team: A guide for managers (2 ${ }^{\text {nd }}$ edition). New Jersey: Prentice Hall.

Thompson, L., \& Fine, G. A. (1999). Socially shared cognition, affect, and behavior: A review and integration. Personality and Social Psychology Review, 3, 278-302.

Tindale, R.S., Smith, C.M., Thomas, L.S., Filkins, J., \& Sheffey, S. (1996). Shared representations and asymmetric social influence processes in small groups. In E. Witte, \& J. Davis (Eds.). Understanding group behavior: Consensual action by small groups (Vol. 1, pp. 81-103). Mahwah, NJ: Lawrence Erlbaum Associates.

Tindale, R.S., Stawiski, S., \& Jacobs, E. (2008). Shared cognitions and group learning. In V. Sessa \& M. London (Eds.). Work group learning: Understanding, improving, and assessing how groups learn in organizations (pp. 73 - 90). New York: Lawrence Erlbaum Associates.

Tindale, R.S., Meisenhelder, H.M., Dykema-Engblade, \& Hogg, M.A. (2001). Shared cognition in small groups. In M. A. Hogg, \& R. S. Tindale (Eds.). Blackwell handbook of social psychology: Group processes (pp.1-30). Oxford: Blackwell.

Tversky, A. \& Kahneman, D. (1973). Availability: A heuristic for judging frequency and probability. Cognitive Psychology, 5, 207-232.

Uitdewilligen, S. (2011). Team adaptation: A cognitive perspective. Maastricht University Press. 
References

Uitdewilligen, S., Waller, M.J., \& Pitariu, A.H. (2013). Mental model updating and team adaptation. Small Group Research, 44, 127-158.

Van den Boosche, P., Gijselaers, W., Segers, M., Woltjer, G., \& Kirschner, P. (2011). Team learning: building shared mental models. Instructional Science, 39, 283-301.

Van den Bossche, P., Gijselaers, W.H., Segers, M., \& Kirschner, P.A. (2006). Social and cognitive factors driving teamwork in collaborative learning environments: Team learning beliefs and behaviors. Small Group Research, 37, 490-521.

Van der Erve, M. (2004). Temporal leadership. European Business Review, 16, 605-617.

Van der Haar, S., Segers, M., \& Jehn, K.A. (2013). Towards a contextualized model of team learning processes and outcomes. Educational Research Review,10, 1-12.

Van der Vegt, G.S., \& Janssen, O. (2003) Joint impact of interdependence and group diversity on innovation. Journal of Management, 29, 729-751.

van der Vegt, G., \& Bunderson, S. (2005). Learning and performance in multidisciplinary teams: The importance of collective team identification. Academy of Management Journal, 48, 532-547.

Van Dyck, C., Frese, M., Baer, M., \& Sonnentag, S. (2005). Organizational error management culture and its impact on performance: A two-study replication. Journal of Applied Psychology, 90, 1228-1240.

van Ginkel, W.P., Tindale, R.S., \& van Knippenberg, D. (2009). Team reflexivity, development of shared task representations, and the use of distributed information in group decision making. Group Dynamics: Theory, Research, and Practice, 13, 265-280.

van Knippenberg, D., \& Schippers, M. C. (2007). Work group diversity. Annual Review of Psychology, 58, 515-41.

van Woerkom, M., \& Croon, M. (2009). The relationships between team learning activities and team performance. Personnel Review, 38, 560-577.

Vashdi, D.R., Bamberger, P.A., \& Erez, M. (2013). Can surgical teams ever learn? The role of coordination, complexity, and transitivity in action team learning. Academy of Management Journal, 56, 945-971.

Waller, M.J., Conte, J.M., Gibson, C.B., \& Carpenter, M.A. (2001). The effect of individual perceptions of deadlines on team performance. Academy of Management Review, 26, 586600 . 
Webber, S.S., Chen, G., Payne, S.C., Marsh, S.M., \& Zaccaro, S.J. (2000). Enhancing team mental model measurement with performance appraisal practices. Organizational Research Methods, 3, 307-322.

Wegner, D.M. (1987). Transactive memory: A contemporary analysis of the group mind. In B. Mullen \& G. R. Goethals (Eds.), Theories of group behavior (pp. 185 -208). New York: Springer-Verlag.

West, M.A. (2000). Reflexivity, revolution and innovation in work teams. In M. M. Beyerlein, D. A. Johnson, \& S. T. Beyerlein (Eds.), Product development teams, Vol. 5 (pp.1-29). Stamford, CT: JAI Press.

West, M.A. (2002). Sparkling fountains or stagnant ponds: An integrative model of creativity and innovation implementation in work groups. Applied Psychology: An International Review, 51, 355-424.

West, M.A., \& Anderson, N.R. (1996). Innovation in top management teams. Journal of Applied Psychology, 81, 680-693.

Wheelan, S.A. (2009). Group size, group development, and group productivity. Small Group Research, 40, 247-262.

Wildman, J.L., Thayer, A.L., Pavlas, D., Salas, E., Stewart, J.E., \& Howse, W.R. (2012). Team knowledge research: Emerging trends and critical needs. Human Factors, 54, 84-111. doi: $10.1177 / 0018720811425365$

Wilson, J.M., Goodman, J.S., \& Cronin, M.A. (2007). Group learning. Academy of Management Review, 32, 1041-1059.

Woehr, D.J., \& Rentsch, J.R. (2003). Elaborating team member schema similarity: A social relations modeling approach. Paper presented at 18th annual Conference of the Society of Industrial Organizational Psychology, 11-13 April, Orlando, FL.

Wong, S.S. (2004). Distal and local group learning: Performance trade-offs and tensions. Organization Science, 15, 645-656.

Wood, S., Michaelides, G., \& Thomson, C. (2011). Team approach, idea generation, conflict and performance. Team Performance Management, 17, 382-404.

Wooldridge, J. M. (2013). Introductory econometrics: A modern approach (5th ed.). Mason, OH: South-Western, Centage Learning.

Yang Z. (2009). Temporal conflict in teams: Antecedents, regulatory mechanisms, and outcomes. PhD thesis, Pennsylvania State University, University Park.

Yukl, G.A. (2010). Leadership in Organizations (7th ed.). Upper Saddle River, NJ: Pearson. 
References

Zaccaro, S.J., Rittman, A.L., \& Marks, M.A. (2001). Team Leadership. The Leadership Quarterly, 12, 451- 483.

Zhou, J. (2003). When the presence of creative coworkers is related to creativity: Role of supervisor close monitoring, developmental feedback, and creative personality. The Journal of Applied Psychology, 88, 413-422.

Zhou, Y. \& Wang, E. (2010). Shared mental models as moderators of team processperformance relationships. Social Behavior and Personality, 38, 4, 433-444. 


\section{VALORIZATION}




\section{VALORIZATION}

The European Commission has recognized "the development of competences, creative and autonomous workers and teamwork" as important assets for both social and economic performance of organizations (Bütikofer, 2013). Recently, in a report that focuses on the creation of a competitive European Union labor market for the 21 st century, the European Commission suggests that "investing in education and in developing skills that respond to the demands of the labor market and society is essential for growth and competitiveness ... [and], that entrepreneurship requires the development of transversal skills such as creativity, critical thinking, teamwork and a sense of initiative which contribute to young people's personal and professional development” (Dlabajová, 2015).

Sustainability and competitiveness depend not only on macroeconomic policies; they also depend on organizational policies and on the dynamics established between individuals, teams, leaders, clients and suppliers that influence organizational performance. Thus, in order to promote the sustainable development of companies and organizations, it is crucial to also understand these micro foundations of organizational competitiveness. This thesis aims to contribute to organizational development by focusing on the team level - as organizations increasingly rely on teams to accomplish complex, demanding, and changing tasks.

Teams have a high capacity to process, store, and use information which makes them particularly suitable to solve problems and make multiple decisions efficiently and in a short period of time. The research reported in this thesis shows that teams achieve their goals efficiently when team members have shared mental models and shared temporal cognitions a common understanding about the important task, team, and temporal aspects of work. This means that team members know what needs to be done, who does what, and when they need to do something. Shared mental models and shared temporal cognitions not only enhance team effectiveness, but they also facilitate important team processes. Shared mental models and shared temporal cognitions, as a cognitive component of teamwork, influence an affective component of team performance by reducing intragroup conflict, and a behavioral component by facilitating team learning behaviors, thereby enabling team members to improve their decision making performance. The findings of this thesis contribute to our understanding of the biggest threat in organizational teams - "the lack of clarity in roles and responsibilities who does what, when, why and with whom" (Salas, personal communication, August 18, 2013). 
Teamwork comes with a number of challenges both for team members and leaders. Team leaders need to motivate and coach their team members to collectively engage in goal directed behaviors. Team members have to be able to motivate themselves to work towards team goals, to and engage in productive relations with their teammates (i.e., without engage in conflicts), and to communicate and coordinate their work effectively. Hence it is pivotal to invest in teamwork skills to ensure that people are able to effectively work together and achieve the desired goals. The results of this thesis suggest that people need to be provided with teamwork skills at an early stage in their academic and/or professional live and throughout their professional career. Importantly, schools, universities, and organizations need to teach their students and employees teamwork skills and competencies, and provide them with strategies that can help them to develop shared mental models and shared temporal cognitions. By showing why and how teams function well and achieve their goals, the results of this thesis translate into social and economic value and are of interest to people responsible for the curricula offered in schools and universities, and of team-based organizations.

Schools and universities should include a number of transversal skills in their curricula, such as teamwork, conflict management, leadership, as these are increasingly important in their social and professional live. In the majority of jobs in our contemporary society, people work in teams, or at least in work groups, and it therefore is important that they have a thorough understanding of the behaviors and communication that facilitate effective cooperation and coordination. As a good example of teaching transversal skills, at Instituto Universitário de Lisboa (ISCTE-IUL) and Maastricht University students have the opportunity to enroll in courses about teamwork, conflict management, communication, and multiculturalism. Teaching those skills from an early stage in people's lives contributes not only to their personal development and to responsible behavior in society, but also to their professional career. Training programs and/or courses in transversal skills should provide students with information about teamwork (including, for instance, team cognition, coordination, communication, learning, and conflict management) that they can learn as well as transfer and apply in their live and in their future work environment. These programs and courses should provide students the opportunity to practice the skills and competencies learned while being supervised by the instructors and teachers who can give feedback to students.

Teams are important in various contexts and team functioning has consequences not only for team members and team leaders, but also for organizations, costumers and patients, 
and even for society. In project teams, when a team does not work well, makes many mistakes and fails to achieve its goals, the client's needs will not be satisfied. As a result, the team's organization may lose the client and may see its reputation decrease. Individual team members may be penalized for their bad performance. When emergency teams, such as firefighters and rescue teams, malfunction, this may lead to dramatic consequences, including material damage and casualties. In healthcare contexts, medical errors may lead to patients' deaths and permanent disabilities, which has consequences for the patients and their families, professionals' careers, reputation of hospitals and professionals. Medical errors also have high financial consequences for hospitals.

A well-functioning team is not only beneficial for social reasons - for the interpersonal relations among people who live in society, and among team members who work together - but also for economic reasons. Teams that work well make less or no mistakes, are more productive and efficient, which increases organizational success and decreases organizational costs. As the findings of the research reported in this thesis show, teams are effective when they have shared mental models and shared temporal cognitions leading them to avoid conflicts, be creative, and learn from each other. Thus, when team members work well together it is more likely that the team achieves its goals, which enables the organization to diminish its costs and increase profits, become more competitive and sustainable. Therefore, employees need to develop knowledge and skills related to effective teamwork not only during their academic live, but also throughout their working life, reinforcing them on a daily-basis while they are working. If employees start to work in a team without having developed the skills needed to do so effectively, the team is likely to fail. In fact, sometimes breakdowns in teams result not from failure in technical skills, but from failure in interpersonal, teamwork, and inter-team skills.

Based on the findings of this thesis, training programs may be developed for professionals, both team members and team leaders, regarding shared mental models, conflict management, team learning and time management. A training program about temporal leadership may be offered in particular to team leaders in order to instruct them regarding optimal ways to help team members prioritize their tasks, to understand when they need to start to work on a task and when they need to finish it by communicating the temporal aspects of work to team members and monitoring them over time. Importantly, team members and leaders should be trained as a team, and in the context in which the team operates; and the training needs to be tailored for the specific team and the individuals that compose that team. 
Teams are different from each other as each team has its own needs and problems. In training programs tailored for a specific team it is possible to develop and promote the aspects that are not well accomplished by team members (i.e., the team weaknesses). These customized training programs allow instructors to help team and team members to deal with their specific problems because "the same pill does not cure everyone diseases". Team leaders should participate in the training programs with all team members as they facilitate the transfer and usage of the knowledge and competencies learned and practiced in the work context. These programs should also give to employees the opportunity to practice the skills and competencies being supervised by the instructors.

The findings of this thesis suggest that a shared understanding about the important aspects of work among team members established at the beginning of the team lifecycle facilitates a fruitful team functioning. Thus, in order to improve team effectiveness and in turn contribute to organizational competitiveness and sustainability, team members and team leaders should participate in planning sessions before the team starts to work. Importantly, these planning sessions used to define, for instance, goals, roles, and responsibilities, need to occur whenever a team starts to work in a new project, even for ongoing teams. Teams should not fall into the trap of starting to work in a new project without discussing and clarifying the main goals of the team, who is responsible for what, what are the main competences and preferences of each team member, how much time they have to work on the tasks, and when the deadlines are.

Planning sessions can be used to facilitate the development of shared mental models in teams. Teams, for instance, can be instructed to collectively create a mind map on paper or on a white board, by connecting the main actions team members need to do and the main information they need to get in order to accomplish the team task. The mind mapping should be visible to all the team members to enable them to adjust their own understanding to that of other team members and adapt their actions accordingly. Importantly, as the findings of this thesis show, shared mental models need to be accurate. Therefore, team leaders task experts or clients should be involved in the mind mapping discussion as much as possible to verify the extent to which the team's mind map matches an external source. If this is not possible, team leaders and task experts could check and validate the mind map later and discuss their ideas and suggestions for improvement with the team afterwards. Planning sessions may be timeconsuming at the beginning of the team lifecycle, but they help the team to focus on the task, reach team goals, and meet deadlines. 
The findings of this thesis show that teams benefit from discussing their working methods, reflecting about what they have accomplished or not, and analyzing errors they might have made in order to avoid them in the future. Besides from team learning behaviors in which teams engage while they are working on the tasks, teams may benefit from participating in debriefing sessions after a team work experience or after the accomplishment of a sub-task. Debriefings are sessions in which team members reflect about a previous and recent experience, discuss the way they worked, identify what they learned, discuss opportunities to improve their work, and plan future tasks or work experiences. Debriefings may be conducted repeatedly over the team lifecycle, or at pre-scheduled moments. Debriefings can improve team effectiveness by providing team members with teamwork skills and competencies, which can improve team performance. In fact, debriefings have been shown to improve team performance by $20-25 \%$, on average (Tannenbaum, Beard, \& Cerasoli, 2013). Further, compared with team training, debriefings are less time-consuming because they last thirty minutes or less, and are less expensive to organizations because only team members and team leaders participate in the debriefing sessions. So, organizations do not need to spend money on hiring a trainer or instructor to manage the sessions. Debriefings can be used to help team members and team leaders to deal with interpersonal conflicts, to develop shared mental models, and to learn from each other. In sum, debriefings aim to provide teams with time to reflect, which can help them to increase their performance in subsequent performance episodes.

The training sessions, planning sessions, and debriefings that can benefit from the findings of this thesis are innovative because they are focused on a, sometimes, neglected level in organizations - the team level. Most of the time, managers, leaders and human resources professionals focus on the individual or organizational level. However, increasingly work is done in teams. What is more, the interventions based on the findings of this thesis are innovative because they are aimed at training team members as a collective in the context where they operate, rather than training people individually. By training a team together, team members have the opportunity to practice the competencies and skills in loco with each other.

Teamwork and team effectiveness is a complex and challenging topic in organizational behavior. The findings of this thesis show that a shared understanding about the central aspects of the team's work is important not only for team effectiveness, but also for team processes by reducing intragroup conflict and facilitating team learning. Importantly, this research can lead to the production of useful, effective, and implementable training 
programs and interventions that can help teams to be effective, and organizations to be competitive and sustainable, which has social and economic value. Therefore, team leaders and managers need to develop research-based practices for improving teamwork in organizations.

\section{REFERENCES}

Bütikofer, R. (2013). Report on reindustrialising Europe to promote competitiveness and sustainability (2013/2006(INI)). Retrieved from European Parliament website: http://www.europarl.europa.eu/sides/getDoc.do?pubRef=//EP//NONSGML+REPORT+A7-2013-0464+0+DOC+PDF+V0//EN

Dlabajová, M. (2015). Report on creating a competitive EU labour market for the 21st century: matching skills and qualifications with demand and job opportunities, as a way to recover from the crisis (2014/2235(INI)). Retrieved from European Parliament website: http://www.europarl.europa.eu/sides/getDoc.do?pubRef=//EP//NONSGML+REPORT+A8-2015-0222+0+DOC+PDF+V0//EN

Tannenbaum, S.I., Beard, R.L., \& Cerasoli, C.P. (2013). Conducting team debriefings that work: Lessons from research and practice. In E. Salas, S.I. Tannenbaum, D. Cohen, \& G. Latham (Eds.), Developing and enhancing teamwork in organizations: Evidence-based best practices and guidelines (pp. 488-519). San Francisco, CA: Jossey-Bass. 

APPENDICES 
Appendices

\section{APPENDIX A - SCALES USED IN THE STUDY REPORTED IN CHAPTER 2}

\section{Team mental models scale}

\section{Task dimension}

1. My team identifies the key-aspects involved in the decision-making; My team ensures that everyone clearly understands our goals

2. My team identifies the key-aspects involved in the decision-making; Team decisions are based on the overall strategy defined for the competition

3. My team identifies the key-aspects involved in the decision-making; My team is extremely effective

4. My team ensures that everyone clearly understands our goals; Team decisions are based on the overall strategy defined for the competition

5. My team ensures that everyone clearly understands our goals; My team is extremely effective

6. Team decisions are based on the overall strategy defined for the competition; My team is extremely effective

\section{Team dimension}

1. Team members work well together; Team members communicate openly with each other

2. Team members work well together; Team members trust each other

3. Team members work well together; My team is extremely effective

4. Team members communicate openly with each other; Team members trust each other

5. Team members communicate openly with each other; My team is extremely effective

6. Team members trust each other; My team is extremely effective

Relationship conflict scale items (Adapted from Jehn, 1995)

1. How much friction is there among members in your team?

2. How much are personality conflicts evident in your team?

3. How much tension is there among members in your team?

4. How much emotional conflict is there among members in your team? 
Team viability scale items (Adapted from Bayazit \& Mannix, 2003)

1. If I could have left this team and worked with another team, I would have. (reverseworded)

2. I wouldn't hesitate to participate on another task with the same team members.

3. If given the choice, I would prefer to work with another team rather than this on. (reverseworded)

Satisfaction item (Adapted from Spector, 1997)

1. How much you are satisfied with all the aspects of your participation in the team. 
Appendices

\section{APPENDIX B - SCALES USED IN THE STUDY REPORTED IN CHAPTER 3}

Shared mental models scale (based on Cannon Bowers and colleagues, 1993)

1. In my team, the team members have a similar understanding about the procedures, strategies and contingency plans involved in decision making.

2. In my team, the team members have a similar understanding of each other's responsibilities, interdependent roles, and communication patterns.

3. In my team, the team members have a similar understanding about the technology, resources, and tools needed to make decisions.

4. In my team, the team members are familiar with the preferences and abilities of each other.

Relationship conflict (Adapted from Jehn, 1995)

1. How much friction is there among members in your team?

2. How much are personality conflicts evident in your team?

3. How much tension is there among members in your team?

4. How much emotional conflict is there among members in your team?

Task conflict (Adapted from Jehn, 1995)

1. How often do people in your team disagree about opinions regarding the work being done?

2. How often are there conflicts about ideas in your team?

3. How often are there differences of opinion in your team?

4. How often are there disagreements within you team about the task you are working on?

Process conflict (Adapted from Jehn, 1997)

1. How often are there disagreements about resource allocation in your team?

2. How often is there conflict in your team about task responsibilities?

3. How often are there disagreements about the way to complete a certain task?

4. How often are there disagreements about who should do what in your team? 
Temporal conflict (Adapted from Yang, 2009)

1. To what extent do team members disagree about time allocation in your work team (how much time to spend on tasks)?

2. To what extent is there conflict about how you should pace task activities in your team?

3. To what extent are there disagreements about how long to spend on specific tasks in your team?

Creativity (Adapted from DiLiello and colleagues, 2011).

Self-perceived creativity

1. I feel that I am good at generating novel ideas.

2. I have confidence in my ability to solve problems creatively.

3. I have a knack for further developing the ideas of others.

4. I am good at finding creative ways to solve problems.

5. I have the talent and skills to do well in my work.

\section{Creative self-efficacy}

1. I feel comfortable trying out new ideas.

2. I have opportunities to use my creative skills and abilities at work.

3. I am invited to submit ideas for improvements in the workplace.

4. I have the opportunity to participate on team(s).

5. I have the freedom to decide how my job tasks get done.

6. My creative abilities are used to my full potential at work.

Team Satisfaction (Adapted from Spector, 1997)

How satisfied are you with...

1. your team?

2. the functioning of your team?

3. your participation in the simulation?

4. the decisions made by your team?

5. communication among your team members?

6. the performance of the team leader?

7. the strategy of your team?

8. the interpersonal relationships among the team members? 
Appendices

\section{APPENDIX C - SCALES USED IN THE STUDY REPORTED IN CHAPTER 5}

\section{Temporal mental model scale}

1. Allocate the time available for each activity; Agreement about time needed to make decisions

2. Allocate the time available for each activity; Planning the work that each team member needs to perform

3. Allocate the time available for each activity; Establishment of a plan of week activities

4. Agreement about time needed to make decisions; Planning the work that each team member needs to perform

5. Agreement about time needed to make decisions; Establishment of a plan of week activities

6. Planning the work that each team member needs to perform; Establishment of a plan of week activities 
Appendices

\section{APPENDIX D - SCALES USED IN THE STUDY REPORTED IN CHAPTER 6}

\section{Shared mental model scale}

\section{Task dimension}

1. Clear understanding of team objectives; Team members understanding regarding the strategy to make decisions

2. Clear understanding of team objectives; Clear understanding about management reports and their implications to make decisions

3. Clear understanding of team objectives; Identical interpretation of information about the Company and the Market

4. Team members understanding regarding the strategy to make decisions; Clear understanding about management reports and their implications to make decisions

5. Team members understanding regarding the strategy to make decisions; Identical interpretation of information about the Company and the Market

6. Clear understanding about management reports and their implications to make decisions; Identical interpretation of information about the Company and the Market

\section{Team dimension}

1. Communicate openly with each other; Trust in each other

2. Communicate openly with each other; Mutual support to perform tasks

3. Communicate openly with each other; Work well together

4. Trust in each other; Mutual support to perform tasks

5. Trust in each other; Work well together

6. Mutual support to perform tasks; Work well together

\section{Temporal dimension}

1. Allocate the time available for each activity; Agreement about time needed to make decisions

2. Allocate the time available for each activity; Planning the work that each team member needs to perform

3. Allocate the time available for each activity; Establishment of a plan of week activities 
Appendices

4. Agreement about time needed to make decisions; Planning the work that each team member needs to perform

5. Agreement about time needed to make decisions; Establishment of a plan of week activities

6. Planning the work that each team member needs to perform; Establishment of a plan of week activities 
ACKNOWLEDGMENTS 


\section{ACKNOWLEDGMENTS}

It could not have been possible to write this thesis and conduct the empirical studies without the support of a group of people and entities. I would like to express my sincere gratitude to everyone that, in different ways, helped me throughout this journey.

To Fundação para a Ciência e a Tecnologia for funding this $\mathrm{PhD}$ project, as well as the period in which I was a visiting doctoral researcher at Maastricht University.

To Ana Margarida Passos for supervising my work with an extraordinary scientific and methodologic rigor, and for teaching me to do serious, honest, and relevant research. I also thank to Ana for supporting me and helping me to overcome the less good moments, as for advising and encouraging me to spend three months at Maastricht University. I am grateful for everything Ana taught me over these years, in my bachelor, master, and $\mathrm{PhD}$. All the knowledge she transmitted me will be reflected in my (and our) future projects.

To Sjir Uitdewilligen for also supervising my work with an extraordinary scientific and methodologic rigor. I thank Sjir for teaching me important strategies to accomplish the tasks and to write papers in the best way possible. I thank Sjir for his meticulous work and feedback in all the stages of my work from the beginning of my journey at Maastricht University until the conclusion of my thesis. I also thank Sjir for giving me the opportunity to be part of a research team and to collect data in Maastricht. Finally, I thank Sjir (and Janneke) for providing me an amazing time in Maastricht.

To Fred Zijlstra for giving me the opportunity to spend some time at the Department of Work and Social Psychology at Maastricht University where I discussed my research, I learned new ways of doing research, and I improved as a researcher.

To António Caetano, Sílvia Silva, Susana Tavares, Silvia Dello Russo, José León Pérez, Aristides Ferreira, Henrique Duarte, Eduardo Simões, and Filomena Almeida. I would like to thank to all of them for giving me support, and feedback throughout this project. I also thank them for giving me the opportunity to work in several projects at Instituto Universitário de Lisboa - ISCTE-IUL.

To Luís Curral, David Patient, Ana Zornoza, and Travis Maynard for giving me feedback and for encouraging me to accomplish this thesis.

To Annika Nübold for her rigorous feedback, suggestions, and work that helped me (us) to transform a good paper in an excellent paper.

To Steffen Sande, Simone Lemmens, and Isa Simons, for collecting the data in the simulation in which I worked with them and Sjir Uitdewilligen, at Maastricht University. 
To SDG for giving me the opportunity to collect data in the Global Management Challenge.

To the "Aquarium fishes". I would like to express my gratitude in particular to Patrícia, Pedro, and Ana Margarida. Together "we practice what we preach". This $\mathrm{PhD}$ journey was a "teamwork" where we learn together and help each other. To my friend Mariana for helping to overcome the challenges I faced in my $\mathrm{PhD}$ as well as in my personal life. To Susana for transmitting me her strength, for her support, and for sharing experiences together. To Sílvia for her support and for the adventures we lived together in Enschede and Maastricht. To Ana Silva, Rita Silva, Rita Rueff, Andreia Correia, Ana Paula Giordano, Pedro José, and Anna Pillo.

To my musketeers Ana, Inês, and Tatiana. Their support and strength were crucial to overcome the most difficult times during my $\mathrm{PhD}$ and personal life. Thanks for celebrating my accomplishments. Je vous aime!

À minha mãe, ao meu pai, à minha irmã e à minha avó pelo apoio incondicional que me deram ao longo deste percurso. Ao meu avô que, apesar de já não estar presente, todos os dias esteve comigo a dar-me força para continuar. Sem este apoio teria sido impossível avançar quando a vontade era desistir, superar o desafio da distância, batalhar perante os obstáculos e alcançar os objetivos. Um agradecimento muito especial a todos ao longo destes últimos meses, em particular ao meu pai, por me terem permitido trabalhar sem estar (demasiado) preocupada com a minha ausência perante o Tomás.

Ao Tomás que me deu, e continua a dar, a maior força do mundo para avançar mesmo quando tenho vontade de largar tudo e ser "só mãe".

Ao André estou eternamente agradecida! Por me ter apoiado incondicionalmente em todos os momentos bons e menos bons deste percurso. Por ter aceitado o adiamento de um sonho para que eu pudesse viver uma experiência única no meu doutoramento. Por termos vivido três meses à distância. Por ser paciente quando eu era impaciente e refilona. Pelos abraços cheios de força que me permitiram carregar baterias quando tudo parecia desmoronar-se. Por acreditar que eu era capaz e que nós iríamos ser capazes. E fuil E fomos! 

Curriculum VitaE 


\section{CURRICULUM VITAE}

Catarina Marques Santos was born on July 26, 1987 in Lisbon, Portugal. She attended Secondary School of Ramada, where she graduated in 2005. She graduated and obtained her Bachelor in Psychology at ISCTE - University Institute of Lisbon, in Lisbon, in 2008. In the same year she started her Master in Social and Organizational Psychology at ISCTE University Institute of Lisbon. During her studies she was an Erasmus student at the Faculty of Social and Behavioural Sciences of Leiden University, The Netherlands. Catarina did her internship for four months in Psicotec, a Human Resources Consultancy, in Lisbon. In 2010 she obtained her M.Sc. in Social and Organizational Psychology. In October 2010, Catarina started her thesis research at the Business Research Unit (BRU-IUL) at ISCTE - University Institute of Lisbon. In September 2011, she was awarded with a doctoral grant (SFRH/BD/79292/2011) from Fundação para a Ciência e a Tecnologia (FCT; Portuguese Foundation for Science and Technology) to her thesis research. In 2013, she was a visiting doctoral researcher at the Faculty of Psychology and Neuroscience of Maastricht University. Currently, Catarina works as a researcher at BRU-IUL at ISCTE - University Institute of Lisbon. After her PhD defense, Catarina will work as a Postdoc at BRU-IUL on a Research Project financially supported by FCT under the title "Team cognition, adaptation and multiteam systems in dynamic environments" (PTDC/MHCPSO/1044/2014).

\section{Publications}

Santos, C.M., Passos, A.M., Uitdewilligen, S., \& Nübold, A. (2016). Shared temporal cognitions as substitute for temporal leadership: An analysis of their effects on temporal conflict and team performance. The Leadership Quarterly. Advance online publication.

Santos, C.M., Passos, A.M., \& Uitdewilligen, S. (2016). When shared cognition leads to closed minds: Temporal mental models, team learning, adaptation and performance. European Management Journal, 34, 258-268.

Santos, C.M., Uitdewilligen, S., \& Passos, A.M. (2015). Why is your team more creative than mine? - The influence of shared mental models on intragroup conflict, team creativity and effectiveness. Creativity and Innovation Management, 24, 645-658. 
Santos, C.M., Uitdewilligen, S., \& Passos, A.M. (2015). A temporal common ground for learning: The moderating effect of shared mental models on the relation between team learning behaviours and performance improvement. European Journal of Work and Organizational Psychology, 24, 710-725.

Standifer, R. L., Raes, A.M.L., Peus, C., Passos, A.M., Santos, C.M., \& Weisweiler, S. (2015). Time in teams: Cognition, conflict, and team satisfaction. Journal of Managerial Psychology, 30, 692-708.

Costa, P. L., Graça, A. M., Marques-Quinteiro, P., Santos, C. M., Caetano, A., \& Passos, A.M. (2013). Multilevel research in the field of Organizational Behavior: An empirical look at 10 years of theory and research. SAGE Open, $1-17 .^{8}$

Santos, C. M., \& Passos, A.M. (2013). Team mental models, relationship conflict and effectiveness over time. Team Performance Management, 19 (7/8), 363 - 385. ${ }^{9}$

Passos, A.M., Silva, S.A., \& Santos, C.M. (2011). Efeitos da orientação para a aprendizagem e segurança psicológica nos conflitos nas equipas [The effects of learning orientation and psycological safety on team conflicts]. Psychologica, 55, 273-290.

\footnotetext{
${ }^{8}$ Equal authorship for the first four authors.

${ }_{9}^{9}$ This work received the award of the outstanding paper of 2014 published in Team Performance Management.
} 


